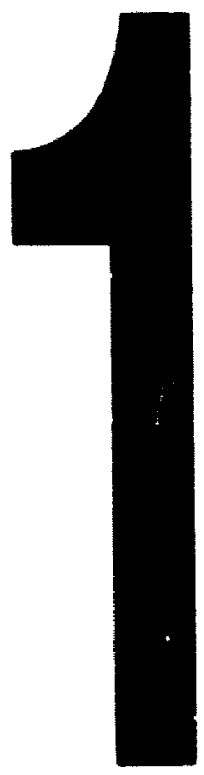

PM-1 3\%"x4" PHOTOGRAPHC MICAOCOPY TAACET

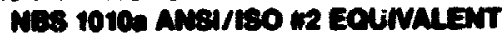

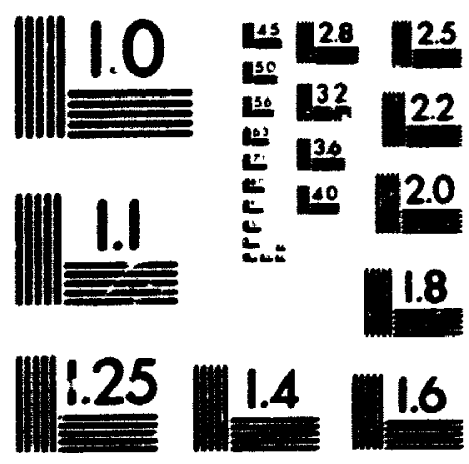

PAECISHON"W RESOUTION TARCETS 
Acquisitions and

Bibliographic Services Branch

395 Wellington Street

Otlawa. Onlarro

KIA ON4
Bibliotheqque nationale du Canada

Direction des acquisitions et

des services bibliographiques

395, rue Wellington

Ottawa (Ontario)

KIA ON4
Your ine volre reterence

Our hile Notre reference

NOTICE

AVIS

The quality of this microform is heavily dependent upon the quality of the original thesis submitted for microfilming. Every eifort has been made to ensure the highest quality of reproduction possible.

If pages are missing, contact the university which granted the degree.

Some pages may have indistinct print especially if the original pages were typed with a poor typewriter ribbon or if the university sent us an inferior photocopy.

Reproduction in full or in part of this microform is governed by the Canadian Copyright Act, R.S.C. 1970, c. C-30, and subsequent amendments.
La qualité de cette microforme dépend grandement de la qualité de la thess soumise au microfilmage. Nous avons tout fait pour assurer une qualité supérieure de reproduction.

S'il manque des pages, veuillez communiquer avec l'université qui a conféré le grade.

La qualité d'impression de certaines pages peut laisser à désirer, surtout si les pages originales ont été dactylographiées à l'aide d'un ruban usé ou si l'université nous a fait parvenir une photocopie de qualité inférieure.

La reproduction, même partielle, de cette microforme est soumise à la Loi canadienne sur le droit d'auteur, SRC 1970, c. C-30, ef ses amendements subséquents. 


\title{
Components and Architectures for an Integrated Wideband FM Transceiver
}

\author{
by \\ Terrence P. Kenny, B.Sc. (Eng.) \\ A thesis submitted to \\ the Faculty of Graduate Studies and Research \\ in partial fulfillment of \\ the requirements for the degree of \\ Master of Engineering
}
Ottawa-Carleton Institute for Electrical Engineering
Depart ment of Electronics
Facul $y$ of Engineering
Carl'ton University
Ottawa, Canada
January, 1994

(1993, Terrence Kenny 
National Library

of Canada

Acquisitions and

Bibliographic Senvices Branch

395 Wellington Street

On'- wa. Ontario

KIAONA
Bibliotheque nationale

du Canada

Direction des acquisitions et

des services bibliographiques

395. nue Wellington

Onawa (Ontario;

KIA ON4
The author has granted an irrevocable non-exclusive licence allowing the National Library of Canada to reproduce, loan, distribute or sell copies of his/her thesis by any means and in any form or format, making this thesis available to interested persons.
L'auteur a accordé une licence irrévocable et non exclusive permettant à la Bibliothèque nationale du Canada de reproduire, prêter, distribuer ou vendre des copies de sa thèse de quelque manière et sous quelque formu que ce soit pour mettre des exemplaires de cette thèse à la disposition des personnes intéressées.
The author retains ownership of the copyright in his/her thesis. Neither the thesis nor substantial extracts from it may be printed or otherwise reproduced without his/her permission.
L'auteur conserve la propriété du droit d'auteur qui protège sa thèse. Ni la thèse ni des extraits substantiels de celle-ci ne doivent être imprimés ou autrement reproduits sans son autorisation.

ISBN $\quad 0-315-92949-9$ 
Name-TERRENCE P. KENNY

Dissertation Abstracts Intemational is arranged by broad, general subject categories. Please select the one subject which most nearly describes the content of your dissertation. Enter the corresponding four-digit cade in the spaces provided.

ELECTEONISS FNGINEERING

SUMECT TERM
$0 / 5 k$ It U.M.I

suner cose

\section{Subject Colegories}

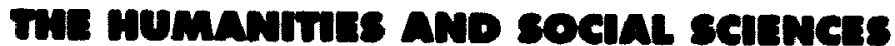

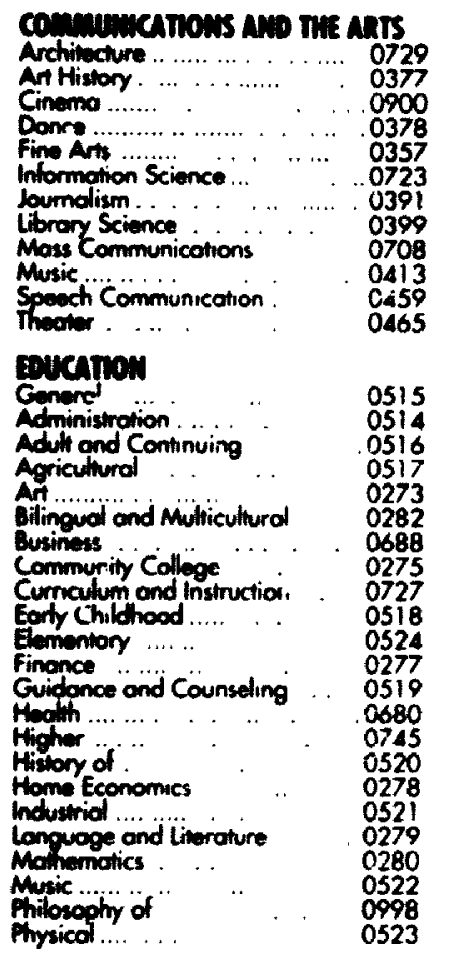

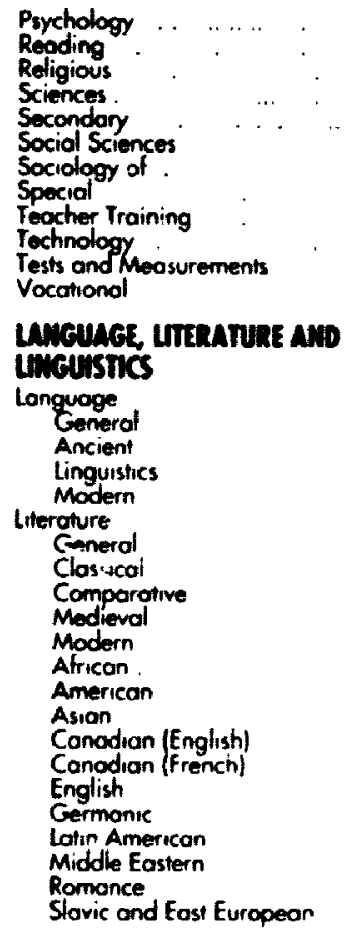

0525

0535

0714

0533

0534

0529

0530

0710

0288

(1)

0679

0289

0291

0401

0294

0295

0297

0316

0591

0305

0352

0355
0593
0311

0312

0315

Stavic and East Europear 0314

\section{Pmosom, newcion and}

\section{Theolos}

Philosophy

Religion

Gonorol

Clergy

Pistory of

Theology

\section{socul sasuces}

Americon Studies

Anthrop slogy

Archireology

Culiural

Business Administration

General

Accounting

Bonking

Manogement

Canodian Studie:

Economics

Generol

Agriculturol

Commerce-Business

Innance

History

Theory

Folklore

Geography

Gerontology

History

Gereral
0422

0318

0321

0319

0322

0323

0324

0326

0310

0272

0770

0454

0338
0385

050 ?

0503
0505
0508

0508

0509

0510

0358

0306

(0)

0578

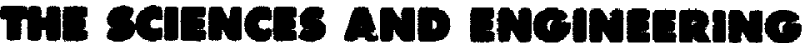

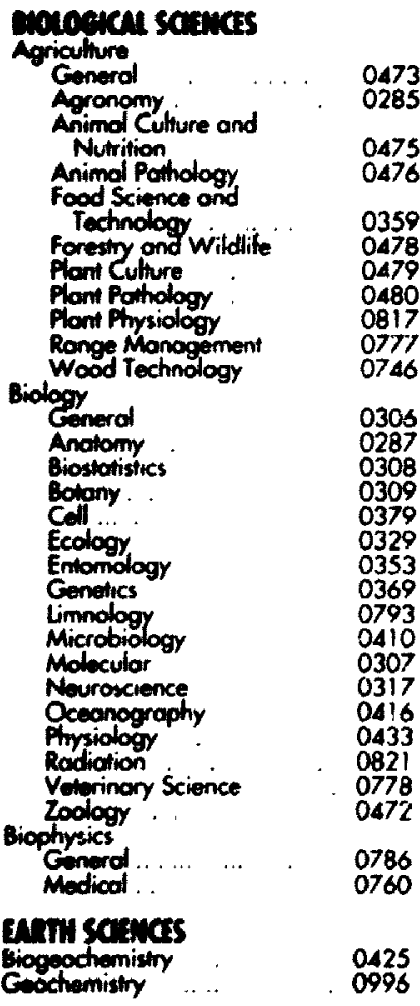

$\begin{array}{ll}\text { Geadesy } & 0370 \\ \text { Geology } & 0372 \\ \text { Geophysics } & 0373 \\ \text { Hydrology } & 0388 \\ \text { Munerology } & 0411 \\ \text { Paleobotony } & 0345 \\ \text { Poleoecology } & 0426 \\ \text { Polontology } & 0418 \\ \text { Poleozoology } & 0985 \\ \text { Polynology } & 0427 \\ \text { Physical Geogrophy } & 0368 \\ \text { Physical Oceonogrophy } & 0415\end{array}$

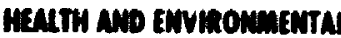
saruas

Environmental Sciences $\quad 0768$ Healith Sciences

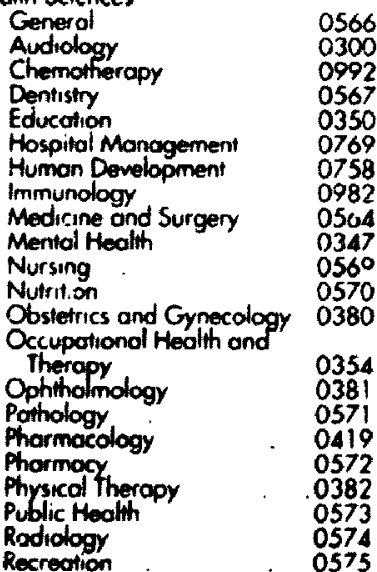

Speech Pathology

Home Economics

\section{Purskell satnces}

Pure Sciences

Chemistry

General

Agricultura

Biochemistry

Inorganic

Nucleor

Phormoceutical

Physical

Polymer

Radiotion

Mothermatics

Physics

Genei al

Acoustics

Astronomy and

Aimospheric Science

Atomic

Eloctronics and Electricity

Elementary Particles and

High Energy

Flido and Plosmo

Moleculor

Nuclear

Opties

Rodiotion

Solid istate

Statistics

Applied Sciences

Applied Mechoni is

Computer Science
0460
0383

0383
0386

0485

0749

0486

0487

0488

0738

0490

0495

0754

0405

0605

0986

0.606

0608

0748

0607

0798

0759

0609

0610

0752

0750
0611

0611

0346

0346
0984

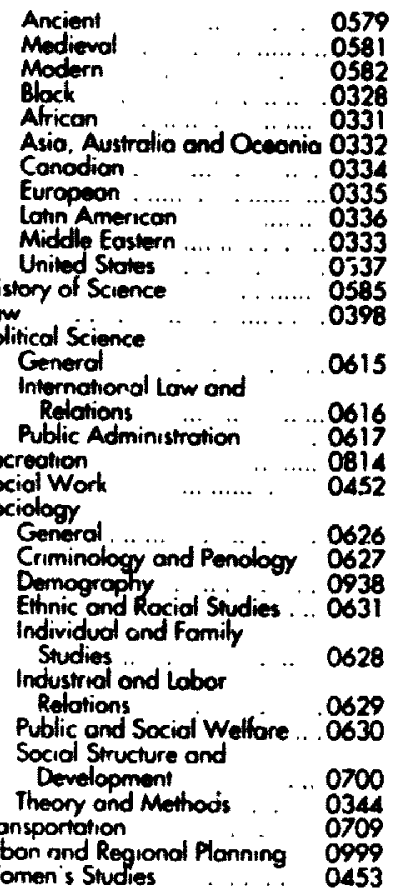

Engyneering

Aerospoce

Agriculiurol

Automolive

Chemical

Civil

Elsctronics and Electrical

Heat anc thermodynomics.

Hydraulic

Morime

Moterials Science

Mechanical

Metallurgy

Muning

Pockaging

Petroleum

Sanilary and Municipal

System Science

Goolectinotogy

Operations Reseorch

Plastics Technology

Esranologr

General

Clinical

Developmental

Experimento

Industrial

Personolity

Peyclobiat

Psyclobiaiogy

Psychometrics
0537 
The undersigned hereby recommend to the Faculty of Graduate Studies and Research acceptance of the thesis,

COMPONENTS AND ARCHITLCTURES FOR AN INTEGRATED WIDEBAND FM TRANSCEIVER

submitted by

Terrence Patrick Kenny, B.Eng.

in partial fulfilment of the requirements for the degree of Master of Engineering.

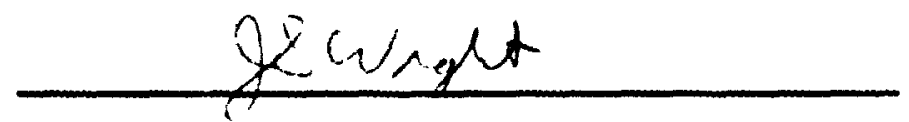

Chair, Department of Electronics

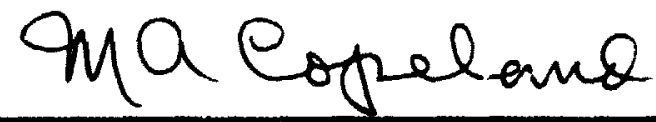

Thesis Supervisor

Carleton University

January 1994 
The information used in this thesis comes in part from the research program of Dr. Miles A. Copeland and his associates in the VLSI in Communications group. The research results appearing in this thesis represent an integral part of the ongoing research program. All research results in this thesis including tables, graphs and figures but excluding the narrative portions of the thesis are effectively incorporated into the research program and can be used by Dr. Copeland and his associates for education and research purposes, including publication in the open literature with appropriate credits. Matters of intellectual property may be pursued collaboratively with Carleton University and Dr. Copeland where and as appropriate. 


\begin{abstract}
This thesis reports on the work performed in the experimental validation of a digitally controlled broadband coded FM concept, based upon the use of a $\Delta \mathbf{\Sigma}$ frequency synthesizer as both a transmitter and a programmable local oscillator, and $\mathbf{a} \Delta \mathbf{\Sigma}$ frequency discriminator as a part of an FM demodulator. The concept and components investigated would support a high degree of VLSI integration in the transceiver. The design and test results of alternative printed circuit board prototypes are presented and a description is given of how these componen. used in the investigation of a broadband digital FM transceiver. Experimental validation of the concept was addressed with the use of a single Fourier component, a digitally encoded sinusoidal FM sequence, varied to produce changes in both frequency deviation and repetition rate. Sideband noise at IF frequency, a product of the conversion of two broadband driven synthesizers, was measured as a function of the repetition rate and peak deviation. The suitability of the output result for $\Delta \mathbf{\Sigma}$ frequency discrimination was evaluated. Finally, the proposed transceiver concept was reviewed in light of these experiments.
\end{abstract}




\section{Acknowledgements}

With respect and gratitude I would like to extend my thanks to Dr. Miles Copeland, my supervisor, for his insight and open handed support throughout the course of this thesis work.

I would like to thank Mr. Tom Riley, my mentor, for his patience and guidance in the work on $\Delta \Sigma$ fractional-N frequency synthesis. I would also like to thank $M r$. Doug Beards for his invaluable assistance in the work on $\Delta \Sigma$ frequency discrimination.

With appreciation I would like to thank Mr. Peter Manashe for his persoral investment and assistance in the preparation of this document.

I would like to acknowledge the financial support of both Carleton University and the Telecommunications Research Institute of Ontario (TRIO).

Finally I would like to thank my wife Pauline, son Matt, and soon to be daughter Caroline Melissa Ann for silently bearing the burden of my nightly and weekend absences. 


\section{Contents}

1 Introduction 1

1.1 Thesis Outline............................ 2

2 A Radio Receiver Architecture 3

2.1 An FM Radio Receiver Design. ................. 3

2.2 A $\Delta \Sigma$ Digital FM Radio Receiver ................. 8

2.2.1 A General Broadband FM Tx/Rx Test Set-up....... 10

2.2.2 A Broadband Coded FM Application........... 12

2.2.3 A Test Bed for Experimental Validation of Broadband Coded FM ........................ 14

2.2.4 Motivation for the Experimental Test Bed Model..... 16

2.3 Figures of Merit for Receiver Performance ........... 17

3 A $\Delta \Sigma$ Frequency Synthesizer 21

3.1 A Fractional-N based PLL .................... 21

3.1.1 The Division Factor. .................... 26

3.1.2 Circuit Realization ..................... 31

3.2 A Third Order Digital $\Delta \Sigma$ Modulator ............... 32

3.2.1 MASH3 Digital $\Delta \Sigma$ Structure. ............... 34

3.2.2 A Third Order $\Delta \Sigma$ Concentrator .............. 39

3.2.3 Circuit Realization ..................... 40

3.2.4 Circuit Measurements. .................. 40

3.3 Frequency Synthesizer Messurement.............. 41

3.3.1 Phase Noise Response. .................. 42

3.3.2 Transient Response.................... 45

3.3.3 Minimu.n Frequency Resolution ............. 46

4 A $\Delta \Sigma$ Frequency Discriminator 48

4.1 Circuit Operation........................ 50

4.2 Circuit Design .......................... 53

4.2.1 The Dual Modulus Divider (DMD) ........... 54

4.2.2 The Phase Frequency Detectur (PFD).......... 55 
4.2.3 Charge pump Circuit Operation .............. 56

4.2.3.1 Charge pump Circuit Design............ 58

4.2.4 The Comparator/Quantizer and Interface Circuit ..... 63

4.3 $\Delta \Sigma$ Frequency Discriminator Circuit Realization.......... 64

5 Experimental Validation of Broadband FM Conversion 70

5.1 Experimental Variables Defined ................. 71

5.2 Distortion Analysis for Broadband FNi Conversion......... 72

5.3 Frequency Synthesizer Characterization using a Sinusoidal Broadband Test Vector...................... 78

5.4 Ideal Broadband Conversion Measurement............ 32

5.5 Broadband Conversion Measurement............... 87

5.6 Qualifying the IF Signal for $\Delta \Sigma$ Frequency Discrimination.... 91

6 Conclusions and Recommendations 96

6.1 Conclusions and Summary $\ldots \ldots \ldots \ldots \ldots \ldots \ldots \ldots \ldots, 96$

6.2 Recommendations for future work on the $\Delta \Sigma$ Fractional-N PLL Frequency Synthesizer. ...................... 99

6.3 Recommendations for future work on the $\Delta \Sigma$ Frequency Discriminator................................. 101

6.4 Recommendations for future work on the Digital FM Transceiver for Broadband Applications. .............. 101

R References 103

A PLL Circuits

B The Third Order Digital $\Delta \Sigma$ Modulator Circuit $\quad B .1$

C $\Delta \Sigma$ Frequency Discriminator and Charge pump Circuits C.1

D Frequency Synthesizer Characterization Plots based upon a D.1 Broadband Sinusoidal FM Test Vector 


\title{
List of Symbols and Abbreviations
}

\author{
AD analog-to-digital \\ $\beta_{F M \quad \text { peak phase deviation }}$ \\ CLB configurable logic block \\ DFT discrete Fourier transform \\ DMD dual modulus divider \\ DPA digital phase accumulator \\ DSP digital signal processing \\ $\Delta f \quad$ sinusoidal FM deviation \\ $\Delta \Sigma \quad$ delta-sigma \\ $\Delta \theta_{e, m} \quad$ synthesizer PLL phase error mismatch \\ ECL emitter coupled logic \\ EMI electro-magnetic interference \\ ENBW equivalent noise bandwidth \\ EÝKOM eraseable and programmable read only memories \\ F linear representation of noise figure \\ FFT fast Fourier transform \\ $f_{m} \quad$ sirusoidal FM rate \\ FM frequency modulation \\ FPGA field programmable gate array \\ $G \quad$ power gain
}


intermediate frequency

IM image frequency

$K_{D}$ discriminator constant

LCA logic cell array

LNA low noise amplifier

LO local oscillator

LPF low pass filter

LSB least significant bit

MASH3 a third order $\Delta \Sigma$ modulator composed of three first order cascaded structures

MEC modulus extension circuit

NF noise figure

OIP $_{3}$ third order intercept point

$P_{I A B}$ $1 \mathrm{~dB}$ compression point

PAL programmable array logic

PBX private branch exchange

PCB printed circuit board

PFD phase-frequency detector

PLL phase locked loop

PN pseudo-random noise

RAM random access memory

RF radio frequency

ROM read only memory

$\boldsymbol{R}_{\boldsymbol{x}}$ receiver

SAW surface acoustic wave

SNR signal-to-noise ratio 


$\begin{array}{ll}\text { SQNR } & \text { signal-to-quantization-noise ratio } \\ T_{x} & \text { transmitter } \\ \theta_{b}(t) & \text { phase variation on the DMD introduced by the test vector } \\ \text { UGBW } & \text { unity gain bandwidth } \\ \text { VCO } & \text { voltage controlled oscillator } \\ \text { VLSI } & \text { very large scale integration } \\ \omega_{n} & \text { natural loop frequency } \\ \zeta & \text { damping coefficient }\end{array}$




\section{List of Tables}

Table Number

Page Number

3.1 Mapping of input to output bits for extended modulus cc.ntrol ...... 29

3.2 PLL loop filter components ...................... 32

5.1 Ideal Broadband conversion results .................. 83

5.2 Broadband conversion measurement results ............. 87 


\section{List of Figures}

Figure Number

Page Number

2.1 Approximation to the magnitude FM spectral density as $\beta_{F M}$ goes to infinity, for the sinusoidal case. ............... 5

2.2 Block diagram of a typical FM receiver ............ 6

$2.3 \quad$ Block diagram of $\Delta \mathbf{\Sigma}$ Digital $F M$ receiver. . . . . . . . . . . 10

2.4 a) A general $T_{x} / R_{x}$ setup for broadband $F M$; b) representation of a broadband sinusoidal FM transmit signal; c) representation of the approximated received signal. ................... 11

2.5 Block diagram of broadband coded FM receiver. ............ 13

2.6 Block diagram of the experimental testbed for broadband coded FM conversion. ............................ 16

2.7 Input power vs. output power response for a non-linear device. ... 19

3.1 A $\Delta \Sigma$ fractional-N based PLL frequency synthesizer. ......... 21

3.2 a) Second orde: active loop filter with a compensating zero; b) equivalent circuit of the filter of part a) ............. 23

3.3 The completer $x$ fifth order loop filter .............. 24

3.4 The dual modulus divider with the modulus extension circuit. ... 27

3.5 Johnson counter divide by 5 cirucit............... 27

3.6 Bit sequence relationships that control the divide ratio........ 28

3.7 Shift register with multiplexed inputs. ............. 30

3.8 Third order digital $\Delta \mathbf{\Sigma}$ modulator with single bit bitstream output. 33

$3.9 \quad$ A third order (MASH) $\Delta \boldsymbol{\Sigma}$ modulator. . . . . . . . . . . . 36

3.10 a) $\mathbf{A}$ first order DPA; b) a first order $\Delta \mathbf{\Sigma}$ modulator. . . . . . . . 37

$3.11 \mathrm{~F}_{7}(\mathrm{z})$, the third order quantization noise shaping function. ..... 38

3.12 Denury to binary (D2B) converter circuit.............. 39

3.13 A third order $\Delta \Sigma$ modulator with single bit bitstream output. . . . . 40

3.14 Simulated power spectral density of complete third order digital

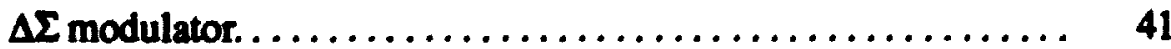


3.15 Measured power spectral density of complete third order digital $\Delta \Sigma$ modulator.

3.16 Single sideband phase noise response of an integer division

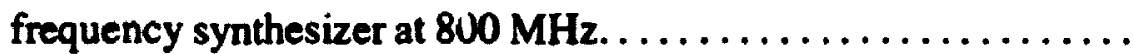

3.17 Single sideband phase noise response of a fractional division frequency synthesizer at $800 \mathrm{MHz} . \ldots \ldots \ldots \ldots \ldots \ldots \ldots .44$

3.18 Transient response for an integer frequency step of $20 \mathrm{MHz}$.... 45

3.19 Transient response for a fractional frequency step of $5 \mathrm{MHz} . \ldots \quad 46$

4.1 a) Equivalent block diagram of a first order $\Delta \Sigma$ frequency discriminator, b) modified block diagram of a first order $\boldsymbol{\Delta \Sigma}$ frequency discriminator. ....................... 50

4.2 Model for the second order $\Delta \Sigma$ frequency discriminator. ....... S1

4.3 a) Block diagram of the second order $\Delta \Sigma$ frequency discriminator, b) waveforms describing correct discriminator operation...... 52

4.4 Timing diagram for the Plessey SP8680.............. 54

4.5 Timing diagram for the Sony PFD, CXB1112Q.......... 55

4.6 Simplified differential charge pump................... 56

4.7 Charge p!ımp model with simplified common-mode feedback.... 57

4.8 Charge pump circuit with feedforward and feedback switching networks, including the common-mode feedback circuit...... 61

4.9 a) Source current circuit; b) current sink circuit. . . . . . . . . . 63

4.10 Bluck diagram of the complete discriminator............. 54

4.11 The reference and feedback signal ECL Pal circuit.......... 64

4.12 Measured timing diagram for the $\Delta \Sigma$ frequency discriminator.... 66

4.13 The power spectral density of the captured discriminator output bitstream.................................. 67

5.1 Block diagram of the experimental testbed for broadband coded

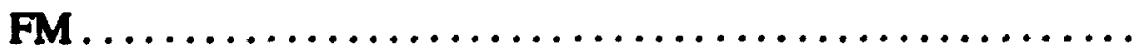

5.2 Block diagram of the model of a fractional-N PLL in terms of its phase components. ............................

5.3 Phasor representation for analysis of IF broadband conversion

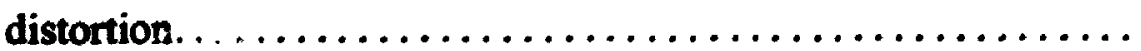

5.4 a) The phsse relationship between the desired and actual transmit frequency, b) the magnitude frequency error between the desired and actual transmit frequency.$\ldots \ldots \ldots \ldots \ldots \ldots \ldots \ldots \ldots$

5.5 Plots of the power spectrum of the individual a) $R_{x}$ and b) $T_{x}$ frequency synthesizers operating in fixed frequency mode....... 
5.6 Plots of frequency versus time for the $R_{x}$ and $T_{x}$ frequency synthesizers using a $10 \mathrm{KHz}$ repetition rate and peak deviation of

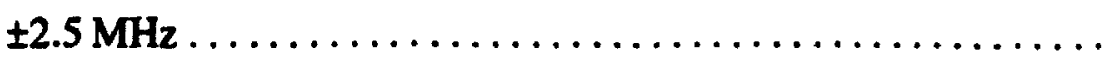

5.7 Plots of frequency versus time for the $R_{x}$ and $T_{x}$ frequency synthesizers using a $1 \mathrm{KHz}$ repetition rate and peak deviation of

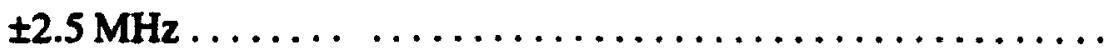

5.8 Plots of the power spectrum for a) the $R_{x}$ and b) the $T_{x}$ frequency synthesizers operating with a peak deviation of $\pm 2.5 \mathrm{MHz}$ and 10 $\mathrm{KHz}$ repetition rate $\ldots \ldots \ldots \ldots \ldots \ldots \ldots \ldots \ldots \ldots \ldots \ldots$

5.9 Block diagram of the experimental testbed used for evaluation of ideal broadband conversion ........................ 83

5.10 Plots of the power spectrum of the $T_{x}$ in self mixing for a frequency deviation of a) $\pm 2.5 \mathrm{MHz}$, b) $\pm 625 \mathrm{KHz}$, and c) \pm 156

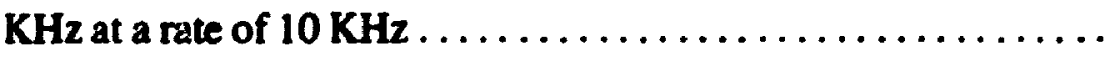

5.11 Plots of the power spectrum of the $T_{x}$ in self mixing for a frequency deviation of $\pm 2.5 \mathrm{MHz}$ and a rate of $1 \mathrm{KHz}$ for wide

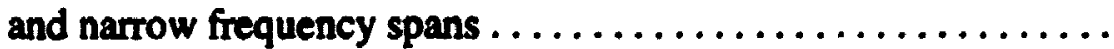

5.12 Plots of the power spectrum of the $T_{x}$ in self mixing for a frequency deviation of a) $\pm 625 \mathrm{KHz}$, and b) $\pm 156 \mathrm{KHz}$ at a rate of $1 \mathrm{KHz}$

5.13 Plots of the power spectrum for the IF frequency using a $10 \mathrm{KHz}$ repetition rate and peak deviations of a) $\pm 2.5 \mathrm{MHz}, b) \pm 625 \mathrm{KHz}$.

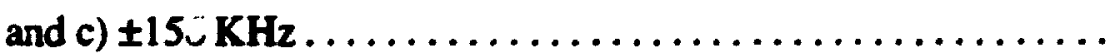

5.14 Plots of the power spectrum for the IF frequency using a $1 \mathrm{KHz}$ repetition rate and peak deviations of a) $\pm 2.5 \mathrm{MHz}$, b) $\pm 625 \mathrm{KHz}$. and c) $\pm 156 \mathrm{KHz} \ldots \ldots \ldots \ldots \ldots \ldots \ldots \ldots \ldots \ldots \ldots$

5.15 The power spectral density of the captured discriminator output bitstream. .......................................

5.16 Power spectral desnity of a DS frequency discriminator bitsream output, reported by Beards and Copeland [BC92]...........

5.17 Block diagram of the broadband conversion set-up including the VO signal levels............................. 94

6.1 Block diagram of a digital FM transceiver............. 97

D.1 Plots of frequency versus time for the $R_{x}$ and $T_{x}$ frequency synthesizers using a $10 \mathrm{KHz}$ repetition rate and pead deviation of

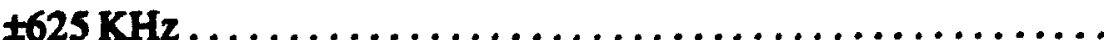


D.2 Plots of frequency versus time for the $R_{x}$ and $T_{x}$ frequency synthesizers using a $1 \mathrm{KHz}$ repetition rate and pead deviation of

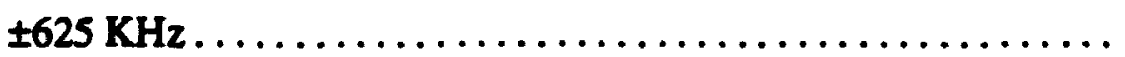

D.3 Plots of frequency versus time for the $R_{x}$ and $T_{x}$ frequency synthesizers using a $10 \mathrm{KHz}$ repetition rate and pead deviation of

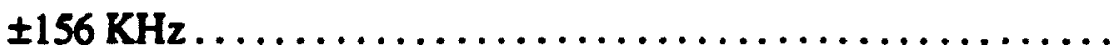

D.4 Plots of frequency versus time for the $R_{x}$ and $T_{x}$ frequency synthesizers using a $1 \mathbf{K H z}$ repetition rate and pead deviation of

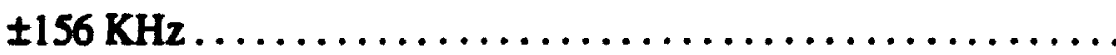

D.5 Plots of the power spectrum for a) the $R_{x}$ and b) the $T_{x}$ frequency synthesizers operating with a peak deviation of $\pm 2.5 \mathrm{MHz}$ and 1

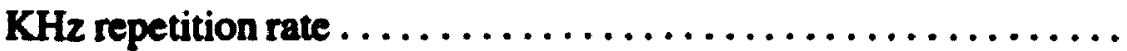

D.6 Plots of the power spectrum for a) the $R_{x}$ and b) the $T_{x}$ frequency synthesizers operating with a peak deviation of $\pm 625 \mathrm{KHz}$ and 10 $\mathrm{KHz}$ repetition rate .

D.7 Plots of the power spectrum for a) the $R_{x}$ and b) the $T_{x}$ frequency synthesizers operating with a peak deviation of $\pm 625 \mathrm{KHz}$ and 1 KHz repetition rate $\ldots \ldots \ldots \ldots \ldots \ldots \ldots \ldots \ldots \ldots \ldots$

D.8 Plots of the power spectrum for a) the $R_{x}$ and b) the $T_{x}$ frequency synthesizers operating with a peak deviation of $\pm 156 \mathrm{KHz}$ and 10 KHz repetition rate .

D.9 Plots of the power spectrum for a) the $R_{x}$ and b) the $T_{x}$ frequency synthesizers operating with a peak deviation of $\pm 156 \mathrm{KHz}$ and 1 KHz repetition rate 


\section{Chapter 1}

\section{Introduction}

The realization of a digital personal communications radio transceiver operating in the $\mathrm{GHz}$ range that meets the specifications of an industry standard is a challenging effort that requires understanding of both component and system level design. Partial motivation for this work is the investigation of altemative component architectures that allow a higher level of VLSI integration, including digital frequency control and a greater level of integrated digital processing. Additional motivation exists for investigation of a broadband pseudorandom coded FM application that may be suitabl: in combatting the effects of multipath fading.

Possibilities for integrated transceiver architectures are explored in this thesis through the construction of two discrete prototype components that can serve as building blocks in higher level radio design. A delta-sigma $(\Delta \Sigma)$ based fractional-N PLL frequency synthesizer [RCK93] is one component that is investigated. Its usefulness as a digitally programmable local oscillator makes it indispensable in the design of multi-channel angle modulated receivers. Equally vital is its usefulness as a digitally controlled angle modulating transmitter. The digital deltasigma modulator driving the synthesizer provides frequency programmability with a resolution dependant upon the number of bits on its input and the reference frequency used. A second component, a digital delta-sigma frequency discriminator [BC92], is also investigated. This component is capable of converting analog frequency deviation into an oversampled digital single bit bitstream whose density of ones is proportional to the frequency deviation of the input signal. This makes the component suitable for a receiver that requires $A / D$ conversion and demodulation of a wide deviation low data rate FM modulated signal.

This thesis studies these two components and proposes an altemative digital radio receiver, as part of a transceiver architecture, which incorporates their features. A 
test bed is developed. which makes use of a broadband sinusoidal FM test vector, for verification of the proposed architecture. The broadband concept is motivated by the need to address the frequency selective fading impairment inherent in mobile radio transceivers. As well, the broadband application appears well suited to the two radio components investigated in this thesis.

\subsection{Thesis Outline}

This thesis is organized over six chapters. Chapter 2 provides an introduction to the architecture of an FM receiver. The capability of digital channel programmability is addressed by the introduction of a $\Delta \Sigma$ frequency synthesizer as a controllable $L O$. Analog to digital conversion and digital data recovery, at the IF stage, are considered with the use of a $\Delta \Sigma$ frequency discriminator. The configuration is suitable for both narrowband FM and broadband coded FM. An architecture for a broadband digitally encoded FM receiver is proposed and an experimental testbed described for evaluation of the broadband coded FM concept. Chapter 3 presents the design and analysis of a digital delta-sigma frequency synthesizer. Quality issues such as quantization noise, phase noise and transient reponse are addressed through the use of simulation and measurement. The design and analysis of a second order $\Delta \Sigma$ frequency discriminator is presented in chapter 4. Measurements of SNR, effective bit resolution and quantization noise shaping indicate the quality of operation. Chapter 5 provides a set of measurements that characterize the operation of the broadband coded FM application presented in chapter 2 and using the componert previously discussed. Chapter 6 presents a conclusion for the work done, and makes some recommendations for continuing work on this type of broadband receiver. 


\section{Chapter 2}

\section{A Radio Receiver Architecture}

In this chapter, the general background of an FM radio receiver design is reviewed. The particular context of an FM radio receiver is then introduced using the alternative components of this thesis.

Radio receiver designs are dependant upon channel impairments and the parameters specified for a particular application. In this chapter, after a review of some basic concepts, a digital FM receiver architecture is presented based upon the use of a digital $\Delta \Sigma$ frequency synthesizer [RCK93] as a controllable LO, and a digital $\Delta \Sigma$ frequency discriminator [BC92] as part of the $\mathbf{F}$ demodulator. The architecture appears well suited for broadband coded applications which continuously move (slither) a carrier signal in a pseudo-random manner over a given spreading bandwidth. The broadband carrier in conjunction with some form of diversity combining in the receiver will provide benefits in overcoming the effects of frequency selective fading, the primary impairment of a wireless environment.

\subsection{An FM Radio Receiver Design}

In FM systems, generation of the transmit signal is usually accomplished with a voltage to frequency converting device (ie. phase locked VCO), where a voltage varying irput $m(t)$ results in a frequency modulated output $v(t)$.

$$
v(t)=A \cos \left[\omega_{c} t+k \int_{-\infty}^{t} m(t) d t\right]
$$


For a sinusoidally varying modulation, signal $v(t)$ can be redefined as

$$
v(t)=A \cos \left[\omega_{c} t+\beta_{F M} \sin \omega_{m} t\right]
$$

Here $\beta_{F M}$, the maximum phase deviation, is referred to as the modulation index. and is a function of the maximum frequency deviation $\Delta f$ and modulation rate $f_{m}$. and is given by

$$
\beta_{F M}=\frac{\Delta f}{f_{m}}
$$

The instantaneous frequency is defined as

$$
f=f_{c}+\beta_{F M} f_{m} \cos \omega_{m} t
$$

This indicates that the instantaneous frequency can always be found within $f_{c} \pm \Delta f$. yet the Fourier expansion of the modulating term of equation (2.2) results in a spectrum consisting of a carrier and a set of sidebands symmetrically spaced on each side of the carrier (ie. Bessel functions). Theoretically, for an FM modulated signal the number of sidebands is infinite, requiring an infinite bandwidth. For all practical purposes for all values of $\beta_{F M}$ this bandwidth is substantially reduced because a greater portion of the total power is contained in a limited number of sidebands. Experimentally it has been shown [TS86] that distortion resulting from bandlimiting an FM signal can be acceptable if at least $98 \%$ of the total power is transmitted through the bandlimiting filter. Therefore, for a sinusoidally modulated FM signal, the required bandwidth $B$ is described by

$$
B=2\left(\beta_{F M}+1\right) f_{m}=2\left(\Delta f+f_{m}\right)
$$

which is the well known Carson's rule.

For wideband FM, generally the Fourier expansion of the frequency modulated waveform cannot be evaluated. The several mechanisms which define the spectrum of an FM waveform [Str79] are the rate of change of the modulating signal and the ratio between amplitude of the modulating signal and the 
instantaneous frequency of the FM signal. Although the instantaneous frequency follows the amplitude of the modulating signal, the spectral density may not result in the sarne pattern. This occurs because the definition of instantaneous frequency and the frequency used in the Fourier transform are not the same. The value of $\boldsymbol{B}_{F M}$ plays a large role in determining the bandwidth requirements for an FM system. If $\beta_{F M}$ is small, then for sinusoidal FM, the spectral content of the modulating signal

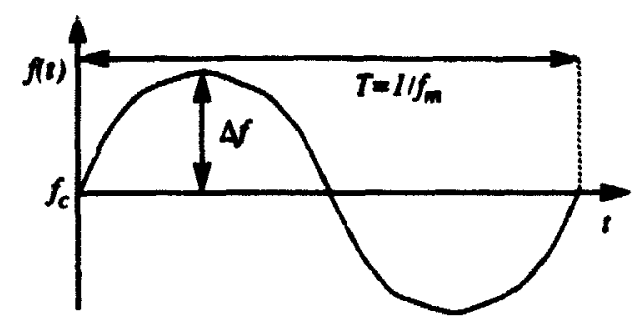

(a)

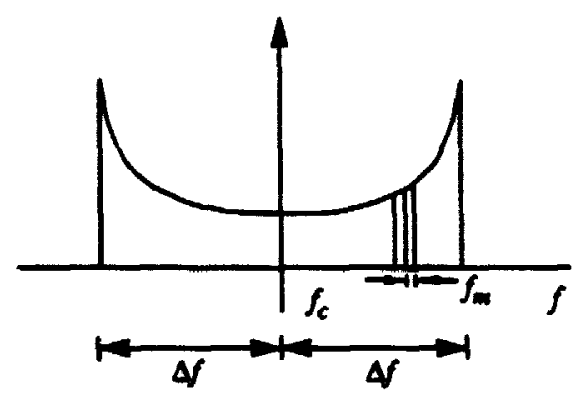

(b)

Figure 2.1 : Approximation to the magnitude FM spectral density as $\beta_{F M}$ goes to infinity, for the sinusoidal case. (reprinted from [St79])

predominantly controls the magnitude of the FM spectral density requiring a bandwidth of $2 f_{m}$. If $\beta_{F M}$ is quite large then amplitude to frequency conversion predominantly controls the amplitude of the spectral density leading to a bandwidth requirement of $2 \Delta f$. Figure 2.1 shows the frequency output signal with respect to time and the expected output spectrum for a wideband sinusoidally modulated FM signal.

Figure 2.2 shows the block Jiagram of a generalized single conversion FM receiver. These type of receivers are designed to provide maximum signal to noise ratio from an RF input that varies widely in signal strength, and may contain adjacent channel interference.

Upon reception at the antenna the RF signal is processed by a low noise amplifier (LNA). The LNA is used as an initial amplifying stage to boost the power of the received signal while minimizing the addition of noise and distortion. Lange gain in the LNA leads to greater overall signal to noise ratio (SNR) if subsequent amplification stages are used in the system. Desireable characterist: $c$ for the LNA 
are large power gain, low noise figure, a wide dynamic range linear transfer function. dynamic stability, reverse isolation, and frequency selectivity (see Figure 2.7). The operating characteristics for the LNA are usually a compromise between these options [KBR80]. The LNA can be preceded by a wideband matching network that reactively matches the source admittance for optimum noise performance. The overall noise figure $F$ (the linear representation of $N F$ ) of the LNA then becomes a function of both the active devices used and the source admittance, and is given by [NS93]

$$
F=F_{\text {min }}+\frac{R_{n}}{G_{g}}\left[\left(G_{o n}-G_{g}\right)^{2}+\left(B_{o n}-B_{g}\right)^{2}\right]
$$

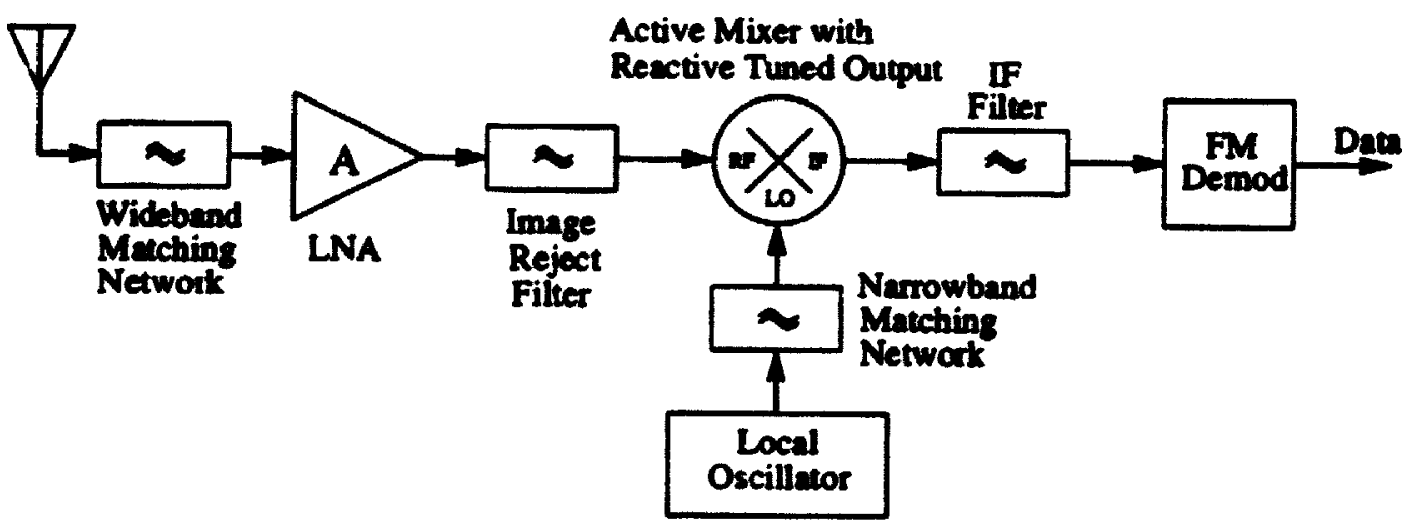

Figure 2.2 : Block diagram of a typical FM receiver.

Here $\left(G_{g}+j B_{g}\right)$ is the generator admittance presented to the input of the LNA, and $\left(G_{o n}+j B_{o n}\right)$ the generator admittance that provides optimum noise performance. $R_{n}$ is the noise resistance and indicates the sensitivity of $F$ to generator admittance, while $F_{\min }$ is an intinsic property of active devices.

For bipolar type amplifiers the factor which determines noise levels is the input impedance match. For each currert/frequency operating point of the amplifier there is an optimum input match $\left(\Gamma_{o p}=R_{o p}+j X_{o p}\right)$ for minimum $F$. If $F$ is presented as a function of reflection coefficients then 


$$
F=F_{\min }+\frac{4 R_{n}}{Z_{o}} \frac{\left|\Gamma_{s}-\Gamma_{o p}\right|}{1+\left.\Gamma_{o p}\right|^{2} \cdot\left(1-\left|\Gamma_{s}\right|^{2}\right)}
$$

and for an optimum input match $\left(\Gamma_{s}-\Gamma_{o p}\right)$ minimum $F$ occurs. Here $\Gamma_{s}$ is the reflection coefficient seen by the input of the LNA.

For a silicon bipolar single transistor or cascode type amplifier the output of the LNA can be matched for optimum power gain using a tank circuit on the collector of the output transistor. This matching network can also incorporate an image reject filter to suppress the noise at the image frequency, thereby ensuring better SNR at the IF stage.

Frequency translation can be accomplished using active or passive mixers. For discrete versions the passive diode mixer is the preferred choice. However, most silicon RF VLSI implementations use the bipolar Gilbert cell because of the inherent conversion gain, isolation and harmonic suppression and because it can be integrated more easily. When preceded by a LNA, under proper matching conditions, the frequency translation can be achieved with greater dynamic range. The RF input on the bipolar mixer sees the output impedance of the LNA in combination with the image reject filter. For a cascode amplifier the output impedance is relatively high, and therefore wideband impedance matching between the LNA and mixer can be achieved by placing a $50 \Omega$ shunt resistor across the high impedance mixer input to Vcc or Gnd. This helps to widen the $3 \mathrm{~dB}$ bandwidth of any parasitic low pass filter present on the mixer input by moving the pole further from DC. This same technique can also be used on the $L O$ input port. If an emitter follower buffer is used at the mixer output then a $25 \Omega$ resistor can be placed in series with its low output impedance to provide some degree of wideband output impedance matching under varying load conditions.

The LO input on the mixer is sourced by an oscillator that is matched for maximum power transfer to ensure minimum reflection. corruption, and harmonic distortion of the oscillator signal. The most important figure of merit for the $\mathrm{LO}$ is double sideband noise. This phase noise on the $L O$ will be translated directly to the IF output at the same ratio as it appeared on the $L$. For multichannel receivers this can present a SNR problem if an adjacent channel, transmitting nearby, has large levels of sideband noise at a channel spacing offset. For a linear oscillator the content of this sideband noise for RF or microwave oscillators is to a great extent 
phase noise, but may also contain I/f (flicker) noise, extemally coupled noise and microphonic (mechanical vibration) noise. The level of phase noise in an oscillator, per unit frequency offset, is a component value that is dependant upon many factors. The phase noise to carrier power ratio for a linear oscillator using a non-ideal amplifier is given by [Rob91]

$$
\left(\frac{N}{P}\right)_{f_{o s}}=\frac{F k T}{P} \cdot \frac{1}{8 Q^{2}} \cdot\left(\frac{f_{c}}{f_{o s}}\right)^{2}
$$

Here $F$ (the linear representation of $N F$ ) is a function of the output power level $P$. Therefore the phase noise levels are a function of the noise figure, the output power level, the loajed $Q$ of the feedback elements in the oscillator circuit and the corner frequency $f_{c}$ of the active device.

The output of the mixer is followed by an IF filter which is used to further suppress adjacent channel interferers or other unwanted signals. The demodulation stage should be presented a signal with the specified in-band SNR and out-of-band suppression. Filters used at this stage are typically high $Q$ components used to pass single channel informstion at the given IF frequency and modulation bandwidth. They may come in the form of SAW, crystal, ceramic, or active tuned filters.

Demodulation of an FM signal can be accomplished with a magnitude limiting frequency to voltage converter where the output voltage is proportional to the frequency deviation of the input signal. However a digital output is desireable to enable greater levels of integrated digital signal processing (DSP).

\subsection{A $\Delta \Sigma$ Digital FM Radio Receiver}

A digital $\Delta \Sigma$ FM radio receiver is defined, in this thesis, based upon the use of a $\Delta \Sigma$ fractional-N PLL frequency synthesizer as a programmable local oscillator, and a $\Delta \Sigma$ frequency discriminator to provide $A D$ conversion and frequency discrimination for the FM demodulation process. A $\Delta \boldsymbol{\Sigma}$ fractional-N PLL frequency synthesizer is a traditional PLL synthesizer that replaces the fixed divider in the feedback path with a dual modulus divider. Using a $\mathbf{\Delta}$ modulated bitstream to control the division modulus allows the synthesis of fractional values of frequencies. The $\Delta \Sigma$ frequency discriminator is a novel architecture by Beards 
and Copeland [BC92] that converts an analog frequency modulated signal to a coarsely quantized digital signal with the modulation signal emoedded as a bit density in the bitstream output.

The digital control on both the $\Delta \Sigma$ frequency synthesizer and the $\Delta \Sigma$ frequency discriminator opens up opportunities for exploring precisely controlled FM architectures for analog/digital radio. By their very nature each componint is suited to wide frequency deviation $(>100 \mathrm{KHz})$, low data rate $(<100 \mathrm{KHz}) \mathrm{FM}$ applications. For this reason the thesis work focuses the investigation on their suitability for broadband FM.

Figure 2.3 shows the block diagram of an FM radio receiver implementation using a $\Delta \Sigma$ frequency synthesizer for the $L O$, and a $\Delta \Sigma$ frequency discriminator for part of the FM demodulation. For narrowband FM the synthesizer can provide the required frequency programmability for a multi-channel receiver, although the issue of narrowband FM will not be addressed in this thesis. The precise frequency control of the synthesizer is dictated by the number of frequency control bits on the $\Delta \Sigma$ modulator input as well as the value of its reference frequency. Alternatively, if the control bits were changed at a rate slower than the transient response of the PLL, then the receiver could be used as a central component for frequency hopping or broadband coded FM applications, i.e.; it could be doing the broadband down conversion. For an $n$ bit interpolated pseudo-random noise (PN) code on the synthesizer $\Delta \boldsymbol{\Sigma}$ modulator input, the useful frequency deviation for broadband movement would be dictated by the useful average bitstream density $\beta$ (fraction of ones per unit section of serial bits) of the output of the $\Delta \Sigma$ modulator. Third order modulators are characterized by useful values of $\beta$ between 0.25 and 0.75 , and therefore the useable programmable bandwidth becomes

$$
\omega_{s s}=2 \pi\left(\beta_{\text {max }}-\beta_{\text {min }}\right) f_{\text {ref }}
$$

where $\beta_{\max }$ and $\beta_{\min }$ are the upper and lower limits of average bitstream density. respectively.

It is interesting to note that the frequency deviation associated with broadband frequency control could be considerably higher than the modulating frequency rate, which is limited by the PLL loop bandwidth. 


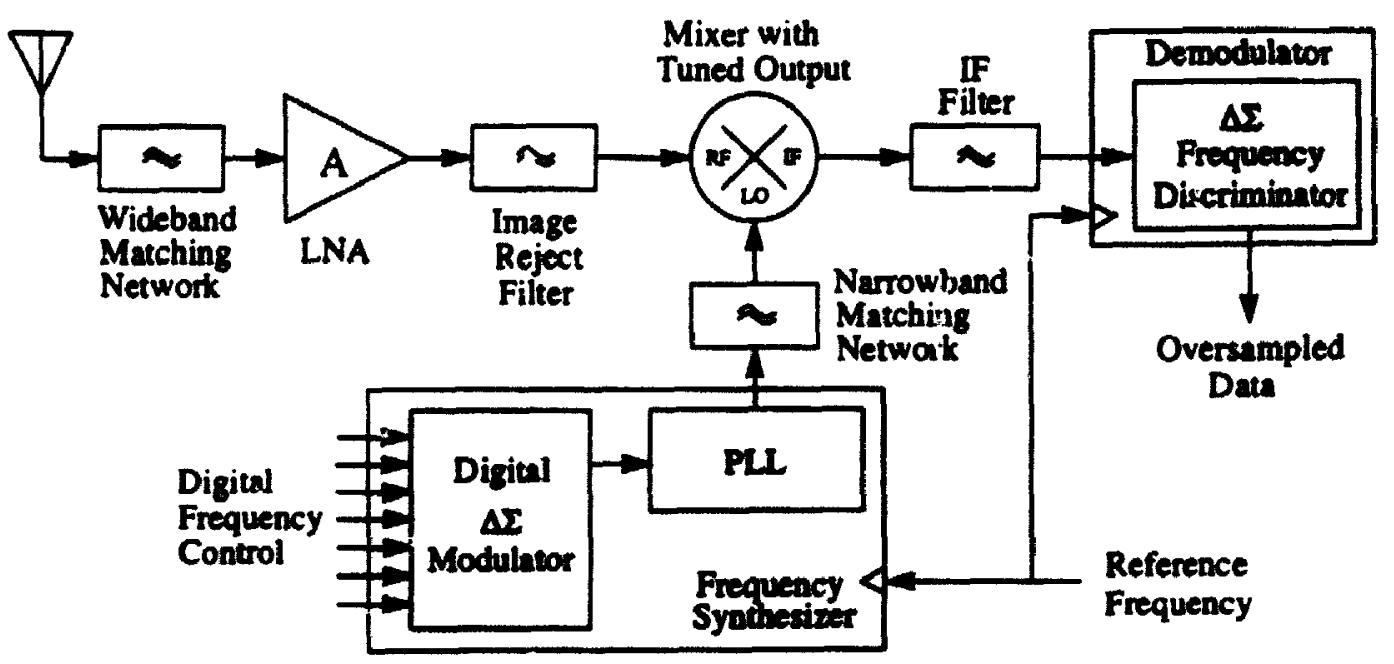

Figure 2.3 : Block diagram of a $\Delta \mathbf{\Sigma}$ Digital FM receiver.

The $\Delta \Sigma$ frequency discriminator converts the analog IF information to a digital bitstream that has high-pass quantization noise shaping. This output signal, an oversampled bitstream, contains the embedded modulation data. The rate of this oversampled output is equal to the reference frequency clock driving both the discriminator and the $\Delta \mathbf{\Sigma}$ modulator. For high reference clock speeds, and bence oversampling rates, wideband frequency demodulation can be accomplished. Post discrimination decimation filtering can be used to remove the quantization noise as well as reduce the oversampled rate to the baseband data rate. The problems of synchronizing the $R_{x}$ frequency synthesizer to the spreading code of the $T_{x}$ signal would be solved in the processing of the digital output of the frequency discriminator and are not dealt with in this thesis. A further thesis project is pursuing this issue [Fi94].

\subsubsection{A General Broadband FM $T_{x} / R_{x}$ Test Set-up.}

Figure 2.4 (a) shows the general block diagram of a broadband FM transmit receive channel which the work in this thesis is aimed at. At the transmitter, data signal $f_{s}(t)$ is added through mixing to broadband spreading signal $f_{b b I}(t)$. producing transmit signal $f_{t}(t)$. If an ideal channel characteristic is assumed, then at the receiver an estimation of the transmitted broadband code $f_{b b 2}(t)$ can be subtracted in a frequency sense from the received signal (i.e. mixed), producing 
signal $f_{I F}(t)$. If both the transmit ard receive broadband spreading signal frequencies are well matched and the addition and subtraction operations are considered linear, then the signal recovered at the IF will be a good approximation of the transmitted signal $f_{s i}(t)$.

$$
f_{I F}(t)=f_{s i}(t)-\left[f_{b b 2}(t)-\tilde{f}_{b b \mathrm{i}}(t)\right] \cong f_{s i}(t)
$$

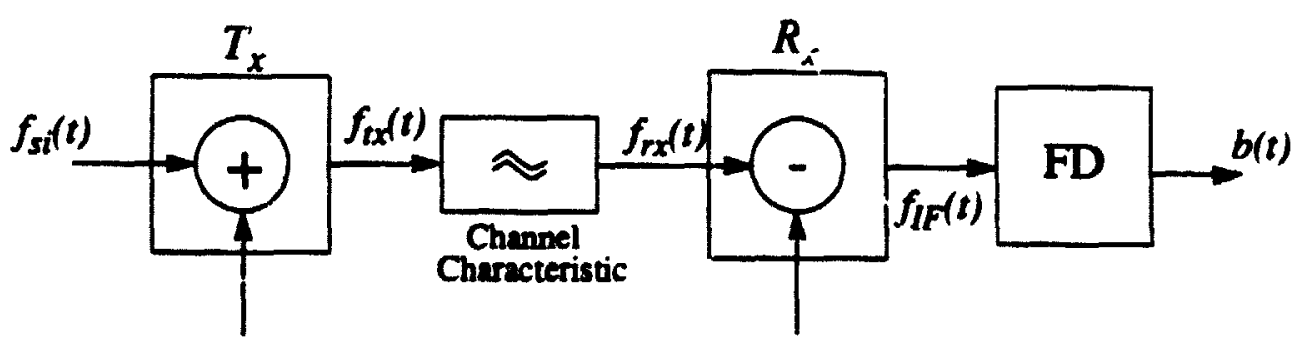

$f_{b b l}(t)$

$$
f_{b b 2}(t)
$$

(a)

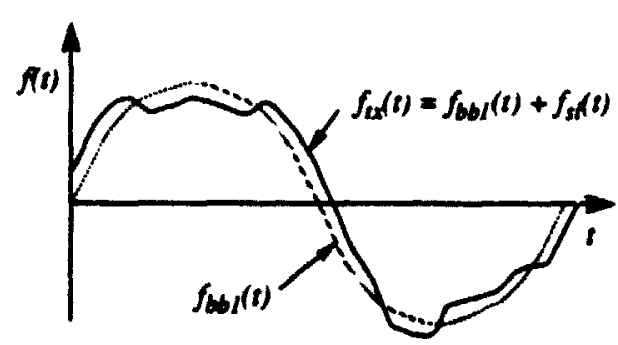

(b)

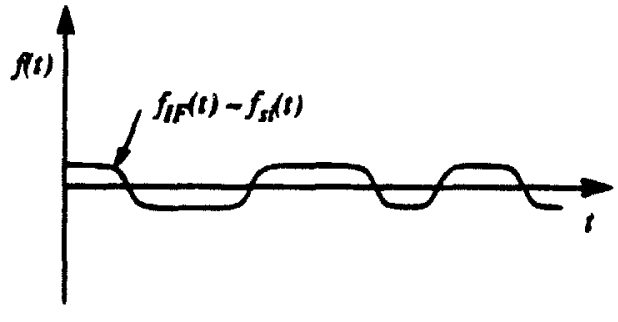

(c)

Figure 2.4 : a) A general $T_{x} / R_{x}$ setup for broadband FM:

b) representation of a broadbend sinusoidal FM transmit signal;

c) representation of the approximated received signal.

If the IF stage is followed by the $\Delta \Sigma$ frequency discriminator, then the discriminator output will be an oversampled single bit bitstream with a bit density proportional to the IF frequency. The discriminator, by the nature of its design, is suited to wide frequency deviation FM signals. Therefore a lage frequency deviation testbed, compatible in frequency range with the discriminator, would allow synchronized testing of the broadband FM concept. 
The fundamental issue requiring investigation is the quality of IF signal resulting from the hroadband mixing of the transmit and receive frequencies. For the proposed architecture to be practical it is essential that the quality of this IF signal be compatable with the signal requirements of the $\Delta \Sigma$ frequency discriminator. which favors wide frequency deviation signals (directly related to SNR).

\subsubsection{A Broadband Coded FM Application}

Figure 2.5 shows the block diagram of a receiver architecture that could be used for broadband coded (frequency slithering) FM applications. The transmit carrier frequency is caused to move over a wide frequency deviation range, sinusoidally or pseudorandomly, except the movement is a continuous interpolation (filtered response) and therefore does not stress the transient behavior of the PLL of the frequency synthesizer in the receiver. This provides the receiver with the opportunity to follow the transmit frequency with a desired fixed frequency offset (to mix to the IF frequency) and, under ideal conditions, with a minimum level of IF sideband noise. The general $T_{x} / R_{x}$ setup shown in Figure 2.4 allows exploration of the feasability of this architecture. For non-strategic broadband frequency environments the complexity of the PN code and the receiver circuit is reduced because detection by unauthorized users, or SNR degradation by intentional interferers, is not an issue. This obviates the use of some form of transinitter preamble to designate channel position and therefore alleviates the burden of coarse acquisition. If a microcellular environment is considered, such as one used for an indoor wireless PBX, then each user could transmit on the same filtered spreading PN code but be assigned a specific phase or bit position within the code. The PBX base station would continuously transmit on all code positions. When a channel or code position was unused, the base station would transmit information about being ready for access as well as the start address for the channel code phase position. In this way each portable receiver, through the use of the coarse synchronization circuit, would then be supplied with a code phase position which would be used by the address generator to pre-align the PN code to the proper sequence. This sequence, in conjunction with the fine synchronization control circuit, would provide an accurate estimate of the transmitted code phase position. The maximum number of users that could be accommodated would be determined by considering the length of the PN code, the number of bits required per user, and the number of bits required to separate each user to ensure minimum cross 


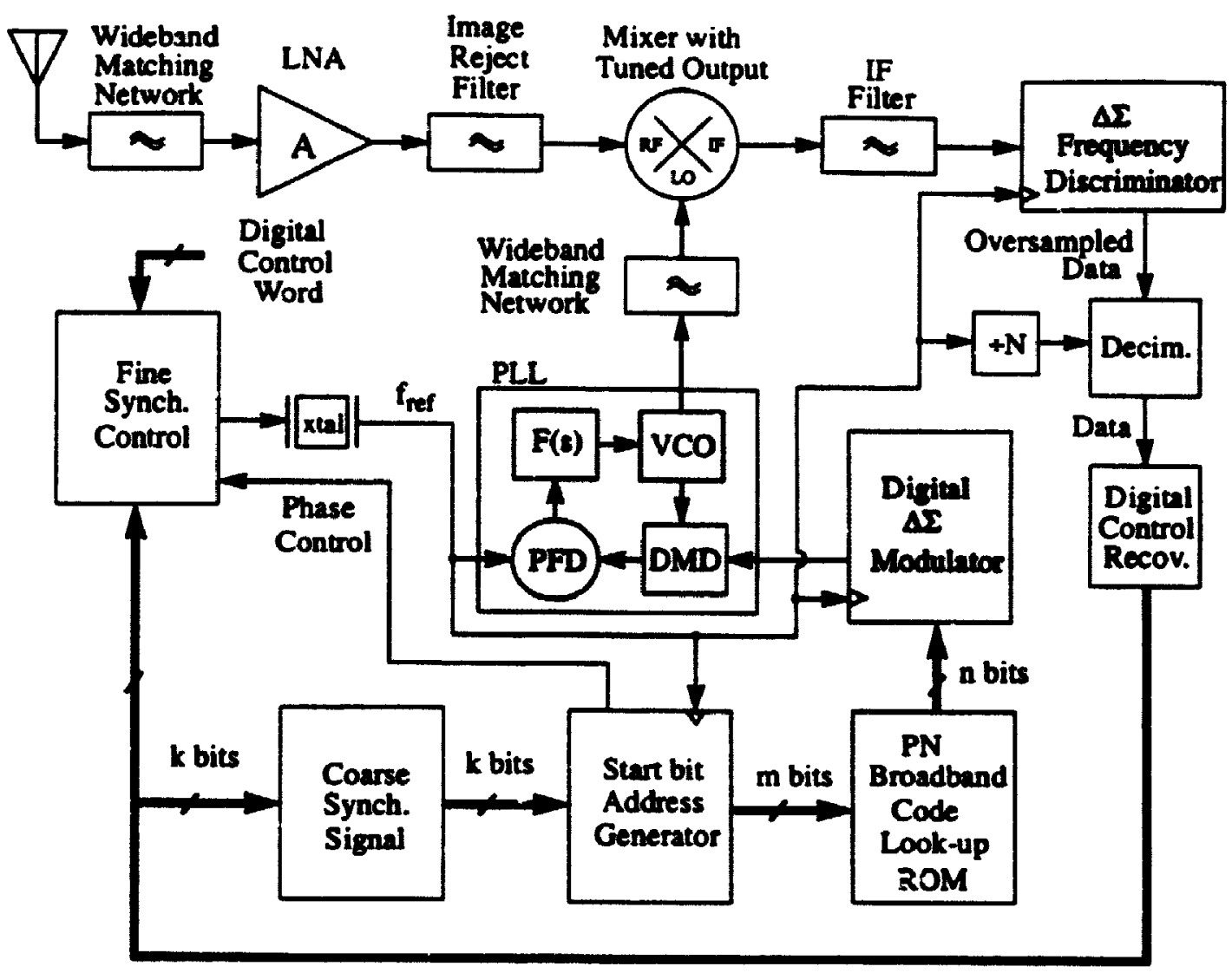

Figure 2.5 : Block diagram of a broadband coded FM receiver.

modulation. Doppler shifts introduced by indoor radio environments tend to be small with respect to digital FM offsets and therefore the frequency bins could be designed to accomodate these shifts. Therefore choice of the spreading PN code lengtin and its rate (with respect to the data modulation rate and frequency deviation) would aid in the design of a receiver with a maximized SNR and a minimized correlation with the nulls of the slow frequency selective fading resulting from the delay spread of the indoor channel. The advantages of a PN code over a simple sinusoidal FM carrier is the resultant uncorrelated sideband noise occurring at the IF. With each user switching on a different position of the same code, care would have to be given to code design to minimize frequency crossover (collisions) of multi-rccess users. Under normal operating conditions most interference would be the result of channels coming on line, or adjacent cell users. 
The use of feedback to the transmitter to control FM deviation of the transmitted data could be used to increase SNR and therefore minimize these effects.

An alternative architecture from the above, using a LNA and $\Delta \Sigma$ frequency discriminator (no broadband conversion mixer), would be possible for cordless telephone applications where a single user per microcell is assumed. The signal correlation would be done digitally after the AD conversion, in the discriminator. The main disadvantage of this architecture is the inability of the discriminator to resolve low level frequency signals in the presence of large magnitude interferers. This drawback limits the general purpose use of this architecture for digital radio.

\subsubsection{A Test Bed for Experimental Validation of Broadband Coded FM.}

In this thesis the design and implementation issues of (coarse and fine) synchronization are not dealt with. Fine synchronization in the testbed is achieved through the use of a common reference signal supplied to both synthesizers and the data generator. Figure 2.6 shows a general block diagram of the testbed structure. The upper left synthesizer in the Figure models part of the transmitter, the bottom right synthesizer part of the receiver. The testbed therefore realizes the general $T_{x}$ ' $R_{x}$ set-up discussed in section 2.2.1 (Figure 2.4), with $f_{b b 1}(t)$ and $f_{b b 2}(t)$ being identical except for analog discrepancies between the two synthesizers. The $T x$ synthesizer uses a direct digital control technique (digital $\Delta \mathbf{\Sigma}$ modulator inputs) for producing the combined broadband frequency and FM modulation frequency $\left(f_{b b l}(t)+f_{b a}(t)\right.$ from Figure 2.4). In the test bed diagram of Figure 2.6 the $T x$ mixer and offset frequency are present only for the purpose of generating a desireable RF transmit frequency (a pragmatic solution for testing), because typically the $T x$ synthesizer would generate broadband frequencies, $f_{T X}$, that are always an IF frequency away from the broadband downconversion frequencies at the receiver, while in the testbed setup identical synthesizers are used which cannot be so separated. The $R x$ synthesizer generetes only a despreading code for the purposes of data recovery. Combined, the $T x$ and $R x$ functions indicate the nature of the proposed channel, although the $T x$ synthesizer is not the only possible way of generating the $T x$ signal. As well, the combination here indicates how a tranceiver might be built, ie: how the transmit function in a transceiver might be achieved. 
This could imply, for instance , full duplex communication in, for example, wireless modem.

The analog differences between the $T x$ and $R x$ synthesizers will lead to 'frequency impairments' at the IF stage which are a function of the bandwidth limitations of the synthesizers, the disparities between the $T x / R x$ VCOs, phase noise levels, and the frequency response of the $R x$ mixer. The test set-up models the consequences of such effects. It may not duplicate exactly how these impairments arrive in the $T_{x} / R_{x}$ combination; for example the final transceiver combination would not require a $T x$ mixer and offset frequency. However by proper programming of the $T \times / R x$ synthesizers in the test set-up it will be possible to demonstrate in a general way the consequences of the impaiments mentioned above. This will help to establish if this is a viable receiver concept. Thus the issue being investigated, through the use of the testbed, is the nature of the signal resulting from the conversion of the exact frequency differences of a combined $f_{s i}(t)$ and $\left.f_{b b l}(t)\right]$ with $f_{b b 2}(t)$ as defined in Figure 2.4 .

One technique for measuring minor frequency variations between the $T x / R x$ synthesizers would be an evaluation of the signal strength of the IF tone (chosen to be $105 \mathrm{MHz}$ ) with respect to sideband noise and distortion. In this case the 105 MHz IF frequency was chosen arbitrarily, although higher frequencies are possible. Thus, assuming the acquisition problem is solved, defining the level of the sideband noise is in effect characterizing the potential quality of the proposed receiver.

Because identical $T x$ and $R x$ synthesizers are used, along with an IF frequency on both of the $T x$ and $R x$ sides, a single test vector can be used to drive both the $T x$ and $R x$ synthesizers. The test vector does not need to contain FM data or need to be moved pseudorandomly in frequency to perform the characterization, but needs to cover the extended frequency range. The experimental test vector chosen to drive both the transmit and receive frequency synthesizers is a single time varying component of a general Fourier series, encoded as adouble precision digital sinusoid. This provided a pragmatic technique, similar to the one used by Sorfleet [Sor91], for testing the broadband concept. A bitstream consisting of double precision $\Delta \mathbf{\Sigma}$ noise shaping code, modulated by a wideband FM sinusoidal vector was produced in software [Ri2] and stored as an ASCII file. Using a bitstream generator card (designed for the XT style bus of an IBM compatable computer), the stored bitstream was loaded from a file on floppy disk to RAM on the 


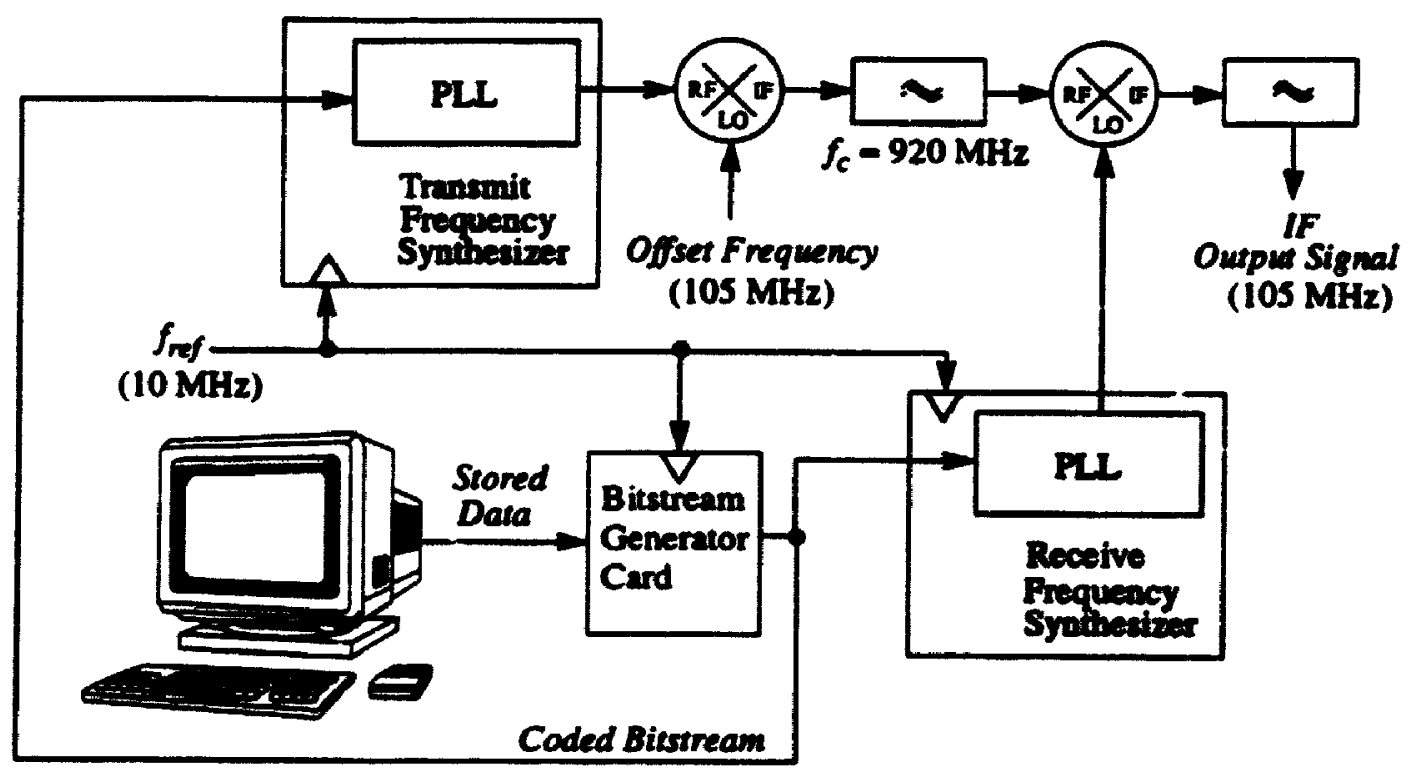

Figure 2.6 : Block diagram of the experimental testbed for broadband coded FM.

generator card and then shifted out serially from the card to the DMD (and MEC) of the frequency synthesizer, synchronized to a master $10 \mathrm{MHz}$ clock. Figure 2.6 shows the block diagram of the testbed. One limitation of this technique is that it is characterized by limit cycles because of the one million bit finite sequence code length. For a $10 \mathrm{MHz}$ clock rate and a $10^{6}$ bit sequence length, a $10 \mathrm{~Hz}$ tone would develop. However, a low level tone of this frequency would not seriously impair testing.

Variation of two independent parameters, the FM repetition rate and FM deviation, allowed the desired characterization of the broadband coded FM concept.

\subsubsection{Motivation for the Experimental Test Bed Model}

In the experimental set-up of Figure 2.6, in order to validate that broadband coded FM is a viable concept, the level of SNR with respect to sideband tones in the final receiver IF signal must be quantified. For the purpose of initially qualifying the broadband FM concept it is not necessary to introduce a set of random data into the experiment. 
Sideband tones in the test set-up and the proposed transceiver concept can arise for several reasons. These include synchronization imperfections between the clocks driving the $T x$ and $R x \Delta \Sigma$ modulators, and impairments in the path between the $T x$ and $R x$ frequency synthesizer outputs. In the test set-up, ideal synchronization has been assumed (by the use of a common reference frequency). In a true receiver some form of fine synchronization is always implied as shown in Figure 2.5; the equivalent of this is provided in the test bed set-up by direct connection. If ideal fine time synchronization for the receiver is assumed (which will be shown to be reasonable) then the path impairments between the $T x$ synthesizer output and the receiver If signal must be qualified, because these impairments will directly affect the sensitivity (minimum detectable sidetone level) of the broadband measurement.

\subsection{Figures of Merit for Receiver Performance}

In this section a review of some front end receiver issues is included for completeness, although the front end requirements are not considered further in this thesis.

Some of the fundamental criteria for evaluating receiver performance include power gain $G$, noise figure $N F$, thind order intercept point $O I P_{3}, 1 \mathrm{~dB}$ compression point $P_{I d B}$, image frequency $I M$, receiver phase noise, and linear (spurious free) dynamic range [Wat86,KBR80]. Other measures of performance can usually be derived from these.

$G$ can be defined as the ratio of the power delivered to the load by the available source power, and is given thy

$$
G=\frac{P_{\text {out }}}{P_{\text {in }}}=\frac{V_{\text {out }}^{2} / R_{L}}{V_{\text {in }}^{2} / 4 R_{S}}=4 \frac{R_{S} \cdot V_{o u t}^{2}}{R_{L} \cdot V_{\text {in }}^{2}}
$$

where $P_{\text {in }}$ is defined as the power across the input impedance $Z_{i n}$ of the amplifier. When $Z_{i n}$ is not equal to $R_{S}$ then maximum power is not transmitted, hence maximum gain cannot be achieved.

$N F$, a measure of noisiness, can be defined as the degradation of signal-to-noise ratio between the input and ouput for a two port device [ZT85]. 


$$
\begin{gathered}
F=\frac{S N R_{i}}{S N R_{0}}=\frac{S_{i} / N_{i}}{\left(G \cdot S_{i}\right) /\left(N_{e}+G \cdot N_{i}\right)}=\frac{N_{e}+G \cdot N_{i}}{G \cdot N_{i}} \\
N F_{d B}=10 \log (F)
\end{gathered}
$$

This can be an indication of additional increases in the noise floor of the system if electronic or thermal noise is considered. In a receiver context. NF then becomes a measure of the degradation of the sensitivity or dynamic range of the system. If a cascade connection of gain stages are involved, such as in a receiver with an active mixer preceded by a LNA and followed by an IF gain stage, then the NF of the latter stages are suppressed by the gain of the preceding stages.

$$
F_{t o t}=F_{L N A}+\frac{F_{\text {Mixer }}-1}{G_{L N A}}+\frac{F_{I F}-1}{G_{L N A} G_{\text {Mixer }}}+\ldots
$$

NF measurement can be accomplished by varying the power level of a noise source connected to the input of the device under test, and then measuring the level of the output noise power using a receiver.

Harmonic and intermodulation distorton are indications of the level of nonlinearity of a component or device. If a power series description for the transfer function of a non-linear device is considesed [PM91] then the output signal is given by

$$
v_{0}=a_{1} v_{i}+a_{2} v_{i}^{2}+a_{3} v_{i}^{3}+\ldots
$$

Harmonic dis urtion results from the passing of a time varying signal $\left(v_{i}\right)$ through a non-linear device. The output spectrum will contain a scaled version of the fundamental signal plus harmonics or second order terms. Intermodulation distortion results from the passing of two or more time varying signals through a non-linear device. The output spectrum will contain both fundamentals and their 


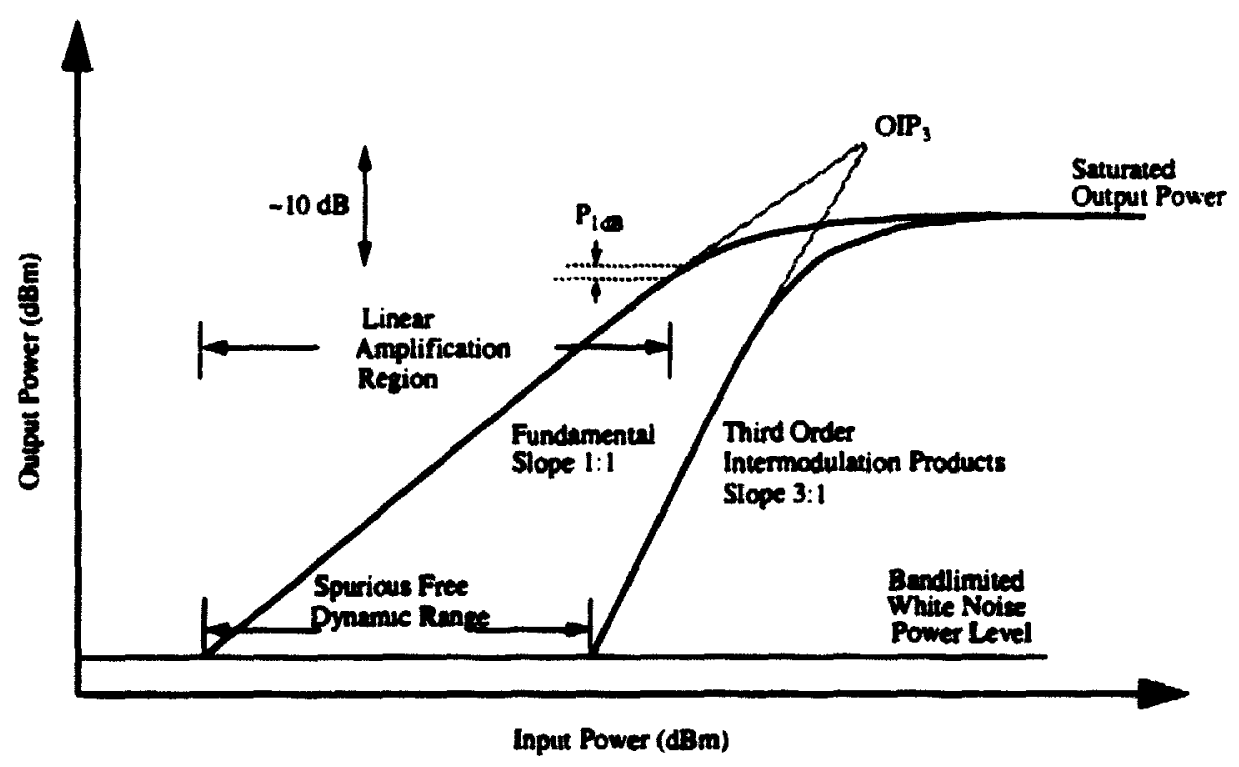

Figure 2.7 : Input power vs. output power response for a non-linear device.

harmonics as well as the beat frequencies of the fundamentals and their harmonics. If the input signals are constrained to be sinusoids and $v_{i}$ is defined as

$$
v_{i}=V_{1} \cos \omega_{1} t+V_{2} \cos \omega_{2} t
$$

then the output spectral terms are $d c, f_{1}, f_{2}, f_{1} \pm f_{2}, 2 f_{1}, 2 f_{2}, 2 f_{1} \pm f_{2}, 2 f_{2} \pm f_{1}, 3 f_{1}, 3 f_{2}$. ... etc. The terms $2 f_{1} \pm f_{2}$ and $2 f_{2} \pm f_{1}$ are the third order intermodulation products, and their difference terms are very important because, being close to the fundamentals, they can fall within the bandwidth of concern and lead to distortion in the output signal. For this reason the resulting power levels of the third onder intermodulation products as a function of the fundamental power levels are very useful measurements for characterizing the quality of receiver components as well as determining appropriate operating power levels. The $\mathrm{OIP}_{3}$, as shown in Figure 2.7, is defined as the point where the third order intermodulation product equals the uncompressed linear output, requires several $R F$ inputs for characterization. The $P_{I d B}$, defined as the point where the measured gain is $1 \mathrm{~dB}$ below the ideal linear gain, requires only a single $R F$ input. Because the $P_{1 d B}$ and $O I P_{3}$ are mathematically related, the easier $P_{I d B}$ measurement is usually sufficient in 
qualifying linearity. The simplified relationship between $\operatorname{OIP}_{3}$ and $P_{1 d B}$ is given by

$$
O I P_{3} \cong P_{1 d B}+10 d B
$$

The image frequency $I M$ is defined as a signal found on the opposing side of the $L O$ from the $R F$ input, and offset from the $L O$ by exactly the IF frequency. Through the act of linear mixing both the $R F$ and $I M$ signals can be translated to the IF band. Signal degradation arising from image frequencies is usually removed by a filter located between the LNA and the mixer. 


\section{Chapter 3}

\section{A $\Delta \Sigma$ Frequency Synthesizer}

In multichannel radio receivers channel selection can be achieved using a frequency synthesizer. Work by Jacquet [JK90] indicates that a fractional division digital PLL, with digitally achieved jitter reduction, is the most suitable approach for monolithic implementation of frequency synthesis in order to achieve high resolution and low complexity.

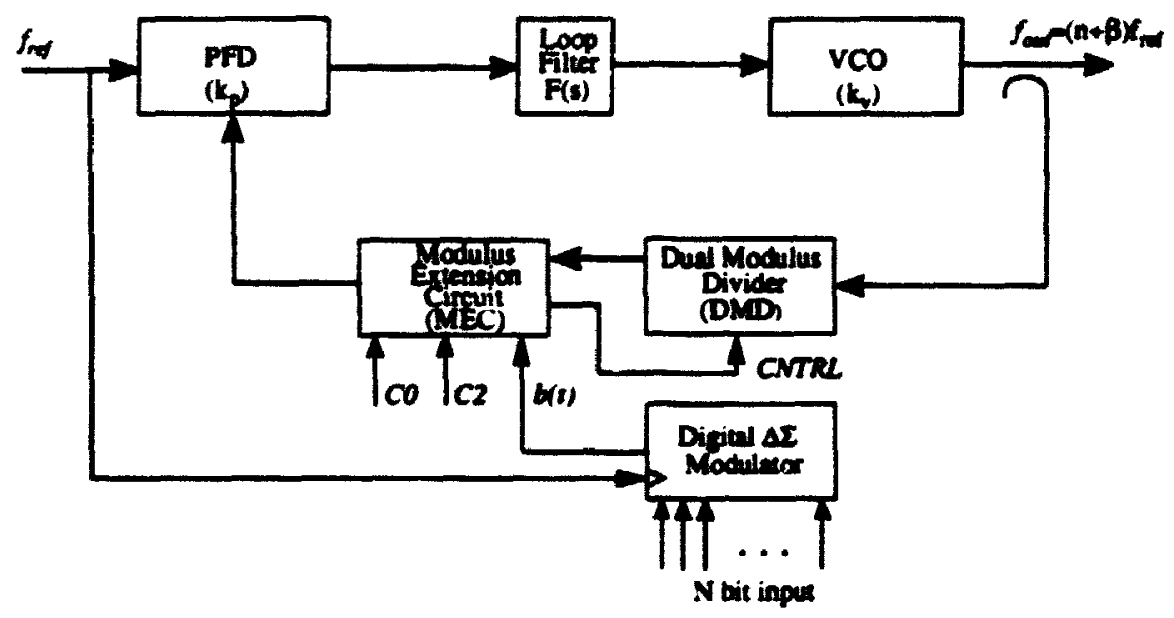

Figure 3.1 : A $\Delta \Sigma$ fr: etional-N based PLL frequency synthesizer

The digital $\Delta \Sigma$ fractional-N PLL frequency synthesizer presented in this chapter is a printed circuit board version of the VLSI implementation currently being researched by Riley [RCK93]. Jitter reduction is achieved using a third order digital $\Delta \Sigma$ modulator to control the modulus division process.

\subsection{A Fractional-N based PLL}


The fractional-N based PLL component of figure 3.1 uses a dual modulus divider (DMD) and a modulus extension circuit (MEC) for prescaling the feedback signal [Zho91]. The average density of ones in the control bitstream driving the DMD provides the capability of generating output frequencies from $n\left(f_{\text {ref }}\right)$ to $(n+l) f_{\text {ref }}$ in fractional steps. The resolution of this step is dependent upon the number of input bits on the $\triangle \Sigma$ modulator. By manipulating the density of ones using the MEC circuit the division capability is extended providing a range of approximately $n\left(f_{\text {ref }}\right)$ to $(n+5) f_{\text {ref }}$ The generalized transfer function of a phase locked loop is given by

$$
\frac{\theta_{0}(s)}{\theta_{i}(s)}=\frac{G(s)}{1+G(s) H(s)}
$$

where, from Figure 2.1

$$
G(s)=\left(k_{p}\right)(F(s))\left(k_{v} / s\right)
$$

and

$$
H(s)=\frac{1}{n+\beta}
$$

Here $\beta$ can be any integer or fraction between 0.25 and 4.75 dependent upon the average density of ones in the control bit stream and the MEC circuit. This density is a function of the dual modulus divide ratio $n, n+1$, and the additional control bits $C_{0}$ and $C_{2}$. The variable $k_{p}$ is a tuning constant dependent upon the type of phasefrequency detector (PFD) employed, and is measured in (volt/rad). As well $k_{v}$, the tuning constant of the VCO, is measured in (rad/sec)/vols. The loop filter F(s) consists of 4 effective stages of filtering with 2 stages generating dominant poles that affect the loop bandwidth.

The output of the PFD is initially low pass filtered using a passive RC structure, whose non-dominant pole (with respect to additional filtering incorporating dominant poles) breaks at approximately 5 times the loop bandwidth [Mot1]. The integrated signal is then processed by an active loop filter, traditionally used in second order PLLs. The equivalent single ended circuit diagram is shown in Figure 3.2 (b) [Mot1,UR83]. 


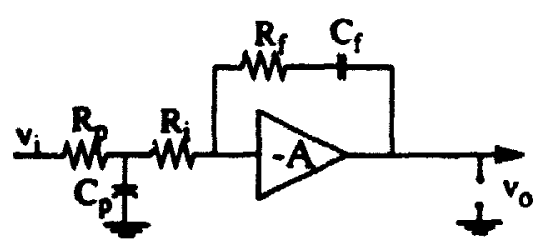

(a)

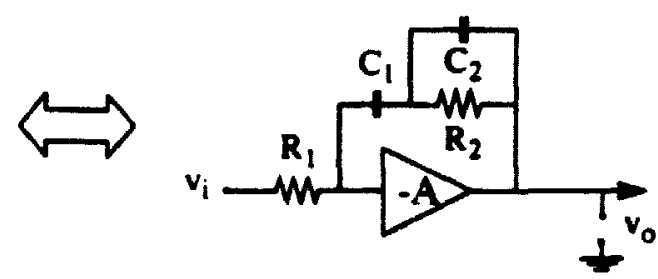

(b)

Figure 3.2 : a) Second order active loop filter with a compensating zero;

b) equivalent circuit of the filter of part (a).

The transfer function of the filter in Figure 3.2 (b) contains two poles and a compensating zero and is given by

$$
F_{1}(s)=-\frac{1}{\tau_{1} s} \times \frac{1+\tau_{2} s}{\left(1+\tau_{3} s\right)}
$$

where

$$
\begin{gathered}
\tau_{1}=R_{1} C_{1} \\
\tau_{2}=R_{2}\left(C_{1}+C_{2}\right) \\
\tau_{3}=R_{2} C_{2}
\end{gathered}
$$

If only the first two stages of filtering are considered then the loop becomes a type 2 third order structure. The type 2 indicates there are 2 DC integrators, and the third order is indicated by the order of the denominator of the open loop transfer function below.

$$
G_{(j \omega)} H_{(j \omega)}=-\frac{k_{v} k_{p}}{(n+\beta) \tau_{1} \omega^{2}}\left[\frac{1+j \omega \tau_{2}}{1+j \omega \tau_{3}}\right]
$$

This equation allows evaluation of both phase and magnitude of the open loop gain as a function of frequency. 
Additional loop filtering, a requirement for suppression of reference frequency feedthrough, was provided using an active second order LPF. An emitter follower configuration, shown in Figure 3.3 (c), was chosen to ensure only a single phase inversion occurs around the loop [Motl,UR83]. The emitter follower also provided a clamping action to the VCO tune voltage by constraining it to within Vcc and ground of the transistor rather than the +12 and -5 volts of the opamp.

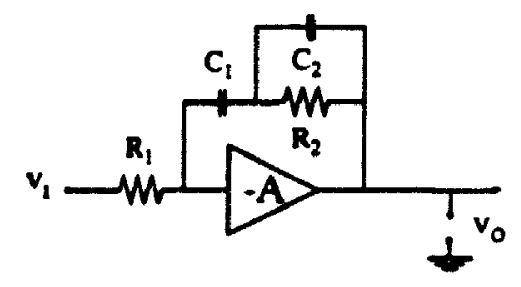

(a)

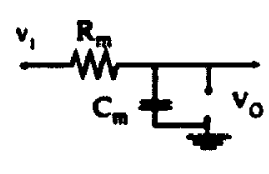

(b)

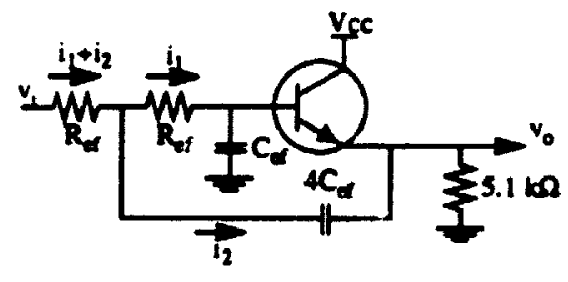

(c)

Figure 3.3 : The complete type 2 fifth order loop filter

The addition of this filter creates a type 2 fifth order losp. From Figure 3.3 (c) we have

$$
\begin{gathered}
v_{o}=v_{i}-\left(i_{1}+i_{2}\right) R_{e f}-i_{1} R_{e f} \\
v_{o}=v_{i}-i_{1} R_{e f}-i_{2} R_{e f}-i_{2} \times \frac{1}{j 4 \omega C_{e f}}
\end{gathered}
$$

assuming a gain of 1 , and no input current to the transistor then additionally

$$
v_{0} \cong i_{1} \times \frac{1}{j \omega C_{e f}}
$$

Rearranging and substituting (3.9) into (3.7) leads to 


$$
i_{2}=\frac{v_{i}-v_{0}-2 j \omega R_{e f} C_{e f} v_{0}}{R_{e f}}
$$

Substituting (3.10) and (3.9) into (3.8) and rearranging provides

$$
F_{2}(j \omega)=\frac{1}{1+2 j \omega R_{e f} \mathcal{C}_{e f}-\left(2 \omega R_{e f} \mathcal{C}_{e f}\right)^{2}}
$$

This results in a Sallen-Key filter with a gain of 1 , and from this second order form normalized to $\omega_{n}$, the cutoff frequency for the emitter follower circuit is found to be

$$
\omega_{c, \text { ef }}=\frac{1}{2 R_{e f} C_{e f}}
$$

An arbitrary choice of $R_{\text {ef }}$ between $1 \mathrm{k} \Omega$ and $100 \mathrm{k} \Omega$ provides the basis for the design of this filter. The magnitude response is unity over the passband with a

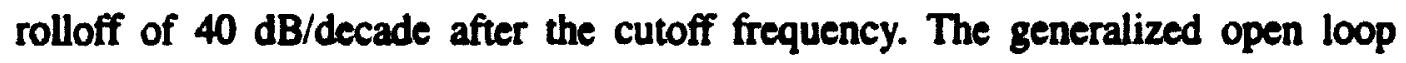
transfer function for the filter can also be described by

$$
F_{2}(j \omega)=\frac{\omega_{n}^{2}}{-\omega^{2}+j 2 \zeta \omega \omega_{n}+\omega_{n}^{2}}
$$

where $\omega_{\mathrm{n}}$ is the natural frequency and $\zeta$ the damping ratio of the filter. If equations (3.6) and (3.13) are multiplied, the complete open loop transfer function for the PLL becomes

$G(j \omega) H(j \omega)=-\frac{\omega_{n}^{2} k_{v} k_{p}}{(n+\beta) \tau_{1} \omega^{2}}\left[\frac{1+j \omega \tau_{2}}{j \omega\left[2 \zeta \omega_{n}+\tau_{3}\left(\omega_{n}^{2}-\omega^{2}\right)\right]+\omega_{n}^{2}-\omega^{2}-2 \zeta \tau_{3} \omega_{n} \omega^{2}}\right]$

This equation can be used to evaluate the magnitude and phase of the open loop transfer function. The use of the emitter follower filter for the additional loop fil- 
tering allows a design that uses two degrees of freedom. Both $\omega_{n}$ and $\zeta$ can be varied independantly for the realization of a stable loop filter. In the actual filter design equation (3.12) was used in the determination of the filter components.

The passive LPF of Figure 3.3 (b) was used to transform the low impedance output of the first stage of filtering to a slightly higher impedance level for better matching to the emitter follower stage. The LPF was also used to effectively isolate the ground planes between the two filter stages. Above the cutoff frequency most of the AC component was coupled to the ground plane of the first stage of filtering. The cutoff frequency of this isolating filter was set to roughly 10 times the loop bandwidth to ensure minimal degradation of loop filter phase margin. For this reason it has not been included in the transfer function of equation (3.14). The transfer function of the matching filter is given by

$$
F_{m}(j \omega)=\frac{1}{1+j \omega \tau_{m}}
$$

where

$$
\tau_{m}=R_{m} C_{m} \cong \frac{1}{10 \times B W_{\text {loop }}}
$$

The equations of this section will be used for component design in a subsequent section that deals with the realization of the phase locked loop circuit.

\subsubsection{The Division Factor}

From the circuit of figure 2.1 the division components shown consists of a single dual modulus divider (DMD) and an additional modulus extenson circuit (MEC) which extends the divider capability from $(n / n+1)$ to $(n / n+5)$. This follows an approach used by Riley [Ri3] and Zhong [Zho91].

The modulus extension is divided into two functions. The first function is a fixed $\Rightarrow$ circuit implemented as a Johnson counter. The second function is a shift register that multiplexes in two control signals and generates a five bit serial sequence that controls the divide ratio of the DMD. The provision of the two input control signals allows the capability of the extended division ratio. Figure 3.4 shows the functional diagram of the DMD with its additional control. 


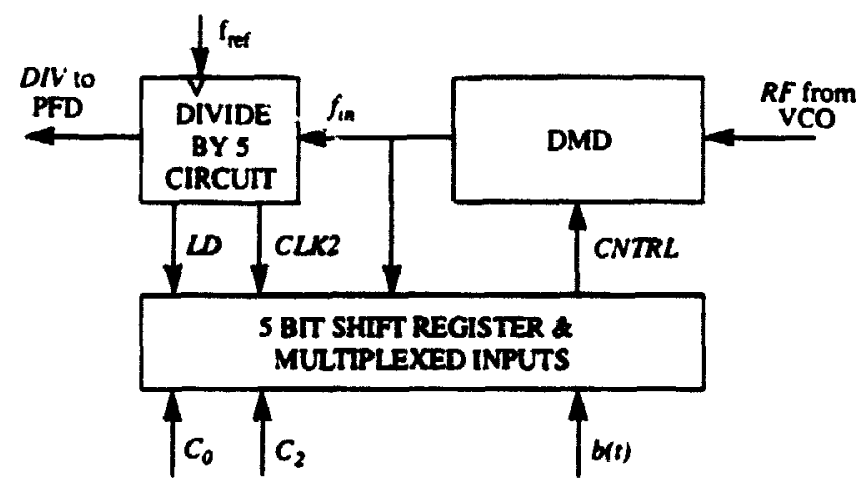

Figure 3.4 : The dual modulus divider with the modulus extension circuit.

Frorn this figure $L D$ and $C L K_{2}$ are $10 \mathrm{MHz}$ signals derived from the $50 \mathrm{MHz}$ input signal $f_{\text {in }}$ of the $\div 5$ circuit. $L D$ is used to load the shift register before the shift sequence begins. $C L K_{2}$ is used to register inputs $C_{0}, C_{2}$ and $b(t) . C_{0}$ and $C_{2}$ are input bits that are multiplexed with $b(t)$ to produce the control signal $C N T L$ which extends the division ratio of the DMD. The signal $b(t)$ is the single bit bistream output of the $\Delta \Sigma$ modulator. Figure 3.5 shows the block diagram of the Johnson counter $\div 5$ circuit.

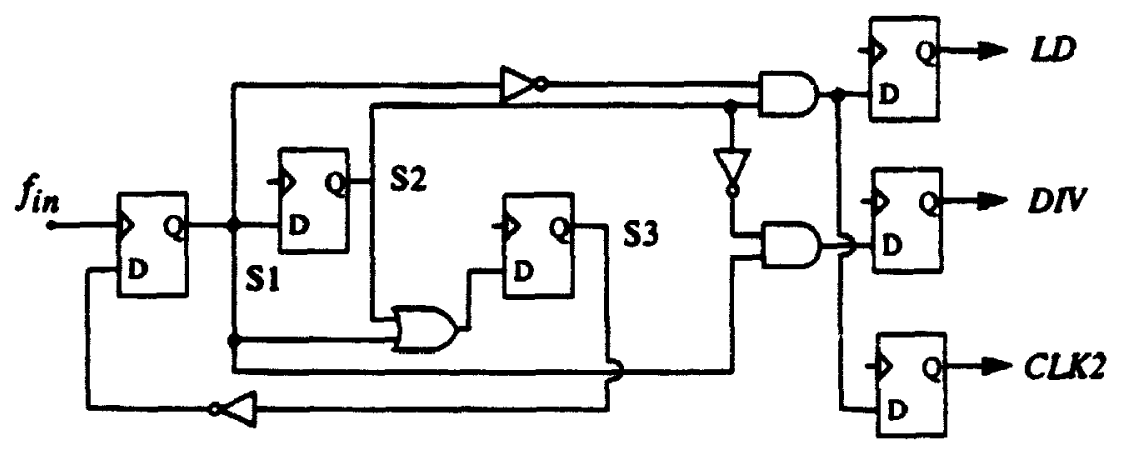

Figure 3.5 : Johnson counter divide by 5 circuit.

Now : fo comen to 세 alp flope

The design of the multiplexed shift register includes features to minimize interference between the bitstream and the divider output. The extended division control of the DMD is based upon a five bit bitstream of data. The design begins with an arbitrary selection of five bit sequences that link the density of ones to the divide 
ratio.

Figure 3.6 shows the timing diagram that relates how bits $D_{0}$ through $D_{4}$ are developed into the $C N T R L$ signal from control signals $C_{0}, C_{l}, C_{2}$, and $b(t)$. The term $C_{l}$ is removed through redundancy and $b(t)$ represents the output bitstream of the $\Delta \Sigma$ modulator, that develops into the extended control signal CNTRL (see Figure 3.7). The rising edge of the DIV signal, which feeds the PFD, must be free of interfering noise and therefore no level changes are allowed to occur between bits $D_{2}$ and $D_{3}$ [MC90]. The density of low bits in $D_{0}$ through $D_{4}$ is directly related to the overall division factor of 80 through $\mathbf{8 5}$. For instance, all five bits high indicates a divide by 80 , while one of five bits low indicates a divide by 81 . The signal $b(t)$, the $\Delta \Sigma$ modulator output, is used to generate bits $b_{1}$ and $b_{2}$ as seen in both Table 3.1 and Figure 3.7. Yet additional control bits $C_{0}$ and $C_{2}$ are required to develop the full five bits required for extended division control. Signals $C_{0}$ and $C_{2}$ originally begin as $C_{0}, C_{1}$, and $C_{2}$, yet redundancy in $C_{0}$ and $C_{1}$ allow $C_{l}$ to be eliminated from the design equations.

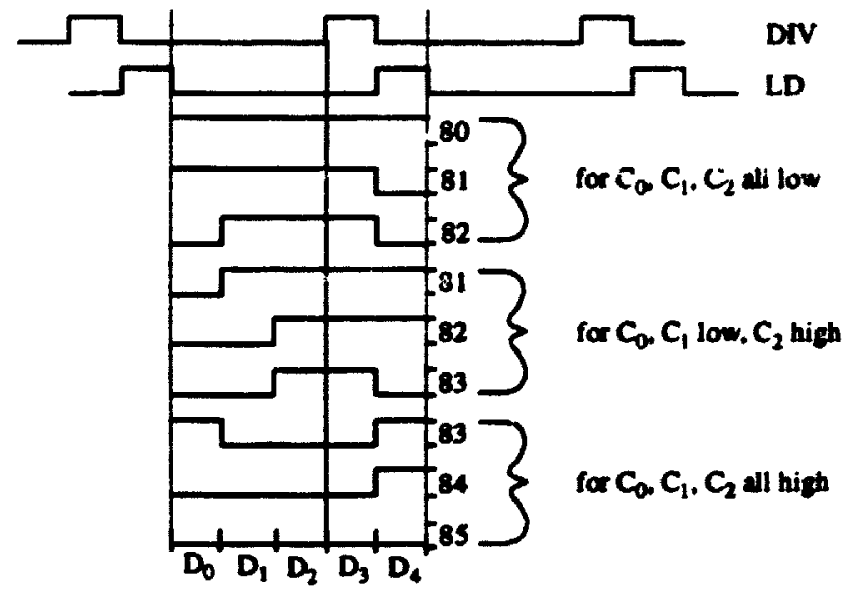

Figure 3.6 : Bit sequence relationships that control the divide ratio.

Table 3.1 shows the mapping of input to output bits. There are 32 possible states based on the five input bits. Only 12 of these states (sections A, B and C) are required to defins: the necessary output states that correspond to a designated division modulus. The resulting modulus extension from the mapping leads to a divsion rarge of 80 through 85 , based upon the original $16 / 17$ dual modulus divider. 
From the mapping of Table 3.1 a set

TABLE 3.1 : Mappling of input to output bits for extended modulus control.

\begin{tabular}{|c|c|c|c|c|c|c|c|c|c|c|c|}
\hline Section & $\mathbf{N}$ & $c_{0}$ & $c_{1}$ & $c_{2}$ & $b_{1}$ & $b_{2}$ & $D_{0}$ & $D_{1}$ & $D_{2}$ & $\mathbf{D}_{\mathbf{3}}$ & $D_{4}$ \\
\hline \multirow[t]{4}{*}{ A } & 80 & 0 & 0 & 0 & 0 & 0 & 1 & 1 & 1 & 1 & 1 \\
\hline & 81 & 0 & 0 & 0 & 0 & 1 & 1 & 1 & 1 & 1 & 0 \\
\hline & 81 & 0 & 0 & 0 & 1 & 0 & 1 & 1 & 1 & 1 & 0 \\
\hline & 82 & 0 & 0 & 0 & 1 & 1 & 0 & 1 & 1 & 1 & 0 \\
\hline \multirow[t]{4}{*}{ B } & 81 & 0 & 0 & 1 & 0 & 0 & 0 & 1 & 1 & 1 & 1 \\
\hline & 82 & 0 & 0 & 1 & 0 & 1 & 0 & 0 & 1 & 1 & 1 \\
\hline & 82 & 0 & 0 & 1 & 1 & 0 & 0 & 0 & 1 & 1 & 1 \\
\hline & 83 & 0 & 0 & 1 & 1 & 1 & 0 & 0 & 1 & 1 & 0 \\
\hline \multirow[t]{4}{*}{$c$} & 83 & 1 & 1 & 1 & 0 & 0 & 1 & 0 & 0 & 0 & 1 \\
\hline & 84 & 1 & 1 & $!$ & 0 & 1 & 0 & 0 & 0 & 0 & 1 \\
\hline & 84 & 1 & 1 & 1 & 1 & 0 & 0 & 0 & 0 & 0 & 1 \\
\hline & 85 & 1 & 1 & 1 & 1 & 1 & 0 & 0 & 0 & 0 & 0 \\
\hline
\end{tabular}

of equations for $D_{0}$ through $D_{4}$ can be generated using $C_{0}$ and $C_{2}$ to differentiate between sections $A, B$ and $C$. Bits $b_{1}$ and $b_{2}$ generate the four states required to define the divide value of $N$ for each section. The reduced Boclean equations, whose assignment is made on the rising edge of $C L K_{2}$, are given by

$$
\begin{gathered}
D_{0}=C_{0} \cdot C_{2} \cdot \overline{b_{1}} \cdot \overline{b_{2}}+\overline{C_{0}} \cdot \overline{C_{2}} \cdot \overline{b_{1}}+\bar{C}_{0} \cdot \bar{C}_{2} \cdot \bar{b}_{2} \\
D_{1}=\overline{C_{0}} \cdot \overline{C_{2}}+\overline{C_{0}} \cdot C_{2} \cdot \overline{b_{1}} \cdot \bar{b}_{2} \\
D_{2}=D_{3}=\overline{C_{0}} \cdot \overline{C_{2}}+\overline{C_{0}} \cdot C_{2} \\
D_{4}=\bar{C}_{0} \cdot \bar{C}_{2} \cdot \overline{b_{1}} \cdot \overline{b_{2}}+\bar{C}_{2} \cdot \bar{b}_{1}+C_{2} \cdot \bar{b}_{2}
\end{gathered}
$$

Equations (3.17) through (3.20) define the five serial output bits of Figure 3.6. These bits are loaded and shifted through a five bit shift register that is clocked by the signal $f_{\text {in }}$, as defined in Figure 3.4. This clock operates at five times the frequency of the clock driving the synchronized inputs of the multiplexer. 


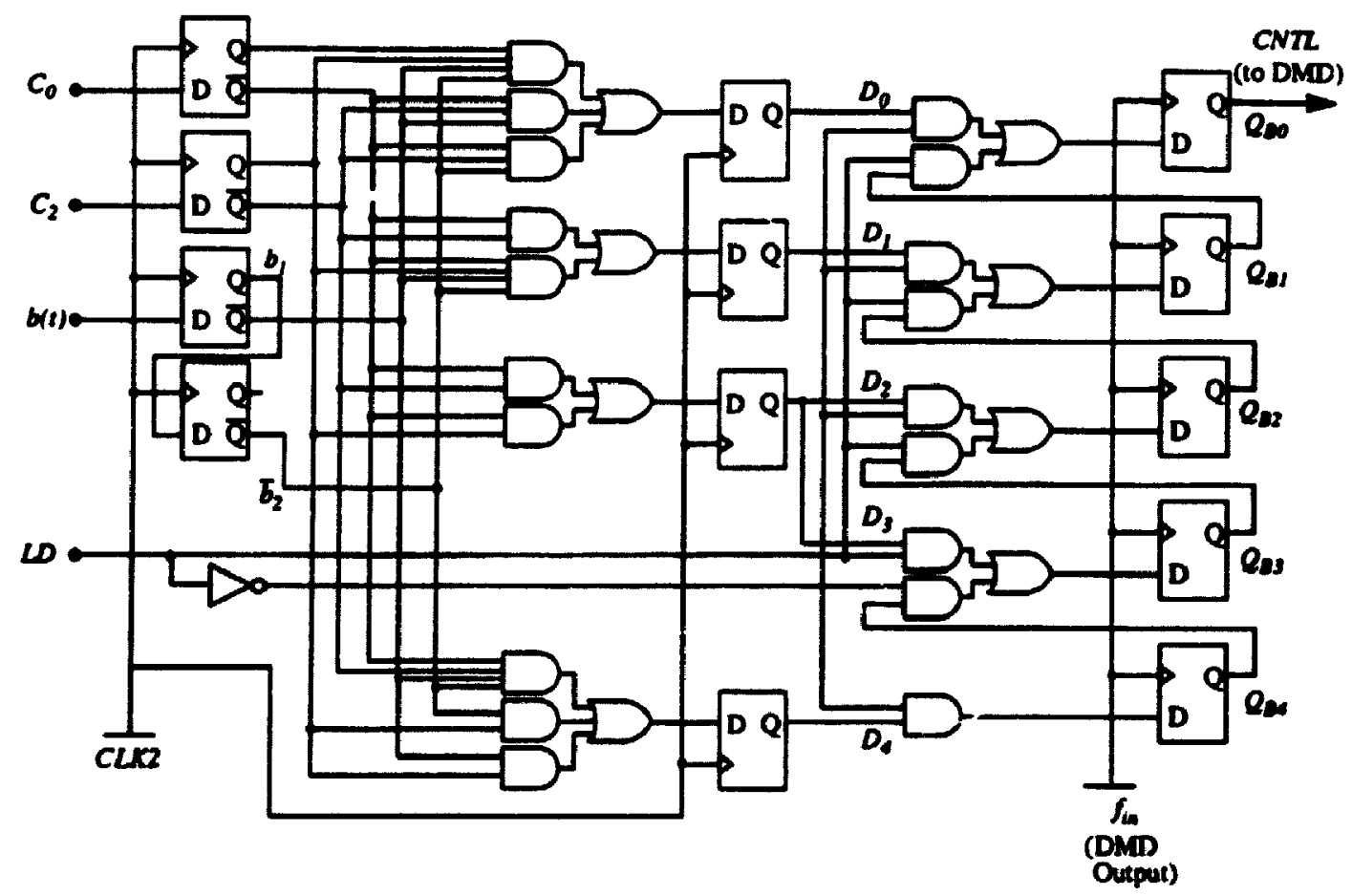

Figure 3.7: Shift register with multiplexed inputs.

Equations $Q_{B O}$ through $Q_{B 4}$, whose assignment is true on the rising edge of $f_{\text {in }}$. define the output bits of the shift register and are given by

$$
\begin{aligned}
Q_{B 0} & =L D \cdot D_{0}+\overline{L D} \cdot Q_{B 1} \\
Q_{B 1} & =L D \cdot D_{1}+\overline{L D} \cdot Q_{B 2} \\
Q_{B 2} & =L D \cdot D_{2}+\overline{L D} \cdot Q_{B 3} \\
Q_{B 3} & =L D \cdot D_{3}+\overline{L D} \cdot Q_{B 4} \\
Q_{B 4} & =L D \cdot D_{4}
\end{aligned}
$$

In equations (3.21) through (3.25) LD, generated by the $\$$ Johnson counter, controls the bit loading and shifting in the shift register. The output of the shift register. $Q_{B O}$, is the bitstream signal $C N T L$ which drives the control pin on the DMD. Figure 3.7 shows the block diagram of the shift register circuit. 


\subsubsection{Circuit Realization}

In the translation of any circuit design from paper to electronics, many aspects of layout and construction must be considered to effect a successful realization. Appendix A contains a set of wiring diagrams and a parts list for the construction of the fractional-N based PLL described in this chapter.

The PLL, a printed circuit board PCB design, was built around a differential VO PFD that is ECL based. The differential connections provide some level of common mode suppression for power supply noise and/or common mode noise present on the device inputs. EMI filters and bypass capacitors were placed at all device power supply pins to further suppress power supply noise. Coupling of the reference frequency input to the PFD was accomplished using a 1:16 transformer to step up the impedance from $50 \Omega$ and to convert to differential mode. This differential signal is then diode limited to provide ECL level swings. Finally the signal is AC coupled and added to an ECL DC bias from the PFD $\left(V_{B B}\right)$ to provide the correct $\mathrm{ECL}$ input.

The differential outputs of the PFD drive the loop filter previously described in this chapter. The final section of this loop filter, the second order emitter follower circuit, was designed empirically to meet the phase stability requirements of the loop. The output signal of this circuit was scaied using a resistive voltage divider to provide attinuation of the loop gain, a requirement due to the large tuning constant $k_{v}$ of the particular VCO (C-616, a standard component produced by Z-communications [Zcom]). The output of the VCO is followed by a $10 \mathrm{~dB}$ resistive pad and amplifier section to provide isolation from unwanted signals and noise in the Inad circuits that might couple back into the VCO and modulate its output signal. The isolated output is then passed through a $3 \mathrm{~dB}$ power splitter to drive an amplifier to provide a $1.8 \mathrm{GHz}$ PLL output, and to drive a 2 prescaler to cut the frequency in half. This signal is amplified again, and then passed through another $3 \mathrm{~dB}$ power splitter to drive an amplifier to provide a $900 \mathrm{MHz}$ PLL output, and to provide an AC coupled signal to the DMD input. This feedback signal is further divided through the extended divider circuit and then converted from a CMOS to an ECL level for comparison by the ECL PFD.

The $\Delta \Sigma$ modulator, working in conjunction with the modulus extension circuit, is connected to the control input of the DMD and determines the divide modulus per reference frequency cycle of the phase locked loop. 
For each group of components a separate retum to ground was used to minimize the creation of inductive ground loops [Mot2]. Microstrip transmission lines of approximately $50 \Omega$ were designed for the critical signal path from the output of the VCO to the input of the DMD. Each of the components on the critical path used $50 \Omega \mathrm{V} / \mathrm{O}$ in order to minimize the amount of mismatch along the signal path.

Table 3.2 lists the: fifth order loop filter component values resulting from the set of design equations presented in section 3.1 of this thesis. The tuning constant of the phase detector, the measured tuning constant of VCO, and the loop bandwidth are shown below.

$$
\begin{gathered}
k_{v}=2 \pi \cdot 76[(M H z) / V] \\
k_{\theta}=\frac{0.8}{2 \pi}[\mathrm{V} / \mathrm{rad}] \\
B W_{3 d B, \text { ClosedLoop }}=80 \mathrm{kHz}
\end{gathered}
$$

TABLE 3.2 : PLL loop filter components.

$\begin{array}{cc}\begin{array}{c}\text { Redistance } \\ (\mathbf{k} \Omega)\end{array} & \begin{array}{c}\text { Capacitance } \\ (\mathrm{ph})\end{array} \\ \mathbf{R}_{1}-1 & \mathrm{C}_{1}-47 \\ \mathbf{R}_{2}=4.2 & \mathrm{C}_{2}-18000 \\ \mathbf{R}_{\mathrm{ef}}=10 & \mathrm{C}_{\mathrm{ef}}=27 \\ \mathbf{R}_{\mathrm{m}}=1 & \mathrm{C}_{\mathrm{m}}=1500\end{array}$

\subsection{A Third Order Digital $\Delta \Sigma$ Modulator}

For the fractional-N PLL frequency synthesizer of Figure 3.1, the modulus input on the DMD requires a controlled bitstream density in order to provide an arbitrary output frequency resolution. A digital $\Delta \Sigma$ modulator, an interpolative coder, is chosen for the control signal because the spectrum of the quantization error exhibits a high pass characteristic. With appropriate antialias filtering and decimation 


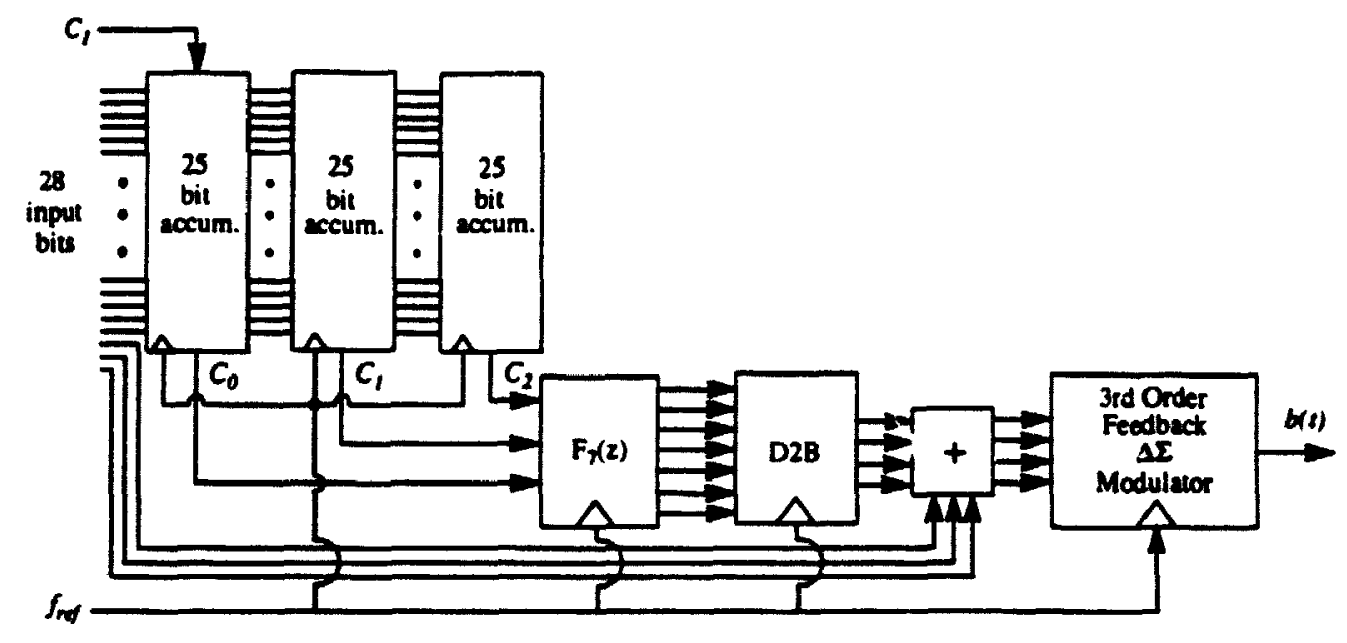

Figure 3.8: Third order digital $\Delta \Sigma$ modulator with single bit bitstream output.

the low frequency component, a representation of the $\Delta \Sigma$ input signal, is transmitted while the high frequency component, primarily quantization noise, is suppresssed.

The order of the zoder is of fundamental importance because of differences in the shape of the output spectrum. Work by Candy \& Benjamin [CB81] indicate that first onder coders generate recurring patterns in the error signal, a function of input level, which result in spurious tones in the output spectrum. At low frequencies. these tones may interfere with baseband signals and lead to bit errors. The only advantage of lower order coders is their unconditional stability.

The output noise spectral density of higher order structures monotonically increases at greater rates per unit frequency (shifts quantization noise to higher frequencies), resulting in greater SNR or bit resolution per given baseband bandwidth. Another advantage of a higher order structure is the interdependance of order, resolution and oversampling ratio. For a given resolution, increasing the order allows a reduction in the oversampling ratio. A grave disadvantage of third order (and higher) cascaded feedback structures is that they are subject to instability. Design of suitable architectures tends to be empirical in nature due to the sensitivity of stability upon initial conditions and scaling factors. Work has been done by Riley [RC92a] and Pinault and Lopresti [PL92] to define the bounds of stability for second order coders. 
In this thesis a third order digital architecture was chosen with 28 input bits and a single bit bitstream output. This effective input bit resolution results in an effective frequency synthesizer output resolution of fractions of a $\mathrm{Hz}$. The requirement for 28 bits in and 1 bit out led to the development of a digital architecture based on the use of a MASH3 type structure [Lon88,WW91], followed by a third order cascaded feedback structure [RCK93] used for concentrating the mutilbit noise shaped signal to a single bit bitstream. Figure 3.8 shows a block diagram of the modulator used to control the division process of the PLL.

\subsubsection{MASH3 Digital $\Delta \Sigma$ Structure}

A MASH3 structure is, by definition, a third order $\Delta \Sigma$ modulator composed of three cascaded first order coders. The advantage of this architecture is that higher order structures are always stable due to the unconditional stability of the fundamental unit, the first order structures.

A $Z$ domain analytical representation of a first order structure, derived by Longo [Lon88], is presented below.

$$
v_{0}(z)=x(z)+\left(1-z^{-1}\right) Q(z)
$$

Here $x(z)$ represents the input signal and $Q(z)$ represents the quantization noise. The product of the integrator gain $\alpha$ and ihe quantizer gain $k$ here are assumed equal to 1, where the quantizer gain adjusts itself to produce unity loop gain. Equation (3.26) shows that the output of the modulator contains a representation of the input signal plus quantization noise. The multiplication factor preceding the quantizer $Q(z)$ is a highpass function indicating that the quantization noise is noise shaped through this feedback action. A definition for the high pass function is given by

$$
\left|1-z^{-1}\right|=2 \sin \left(\frac{\pi f}{f_{s}}\right)
$$

where 


$$
z=e^{j \omega T}
$$

From equation (3.27) an increase in the sampling frequency $f_{s}$ decreases the magnitude of the high pass function, leading to a greater signal-to-quantization-noise ratio SQNR.

If a third order structure consisting of three single stages cascaded, as shown in Figure 3.9, is considered, then from Longo [Lon88] the output signal from each stage is

$$
\begin{gathered}
D_{1}(z)=x(z)+\left(1-z^{-1}\right) Q_{1}(z) \\
D_{2}(z)=z^{-1}\left(\alpha_{1} H_{1}-F_{1}\right) x(z)+z^{-2}\left(F_{1}-\alpha_{1} H_{1}\right) Q_{1}(z) \\
-z^{-1} F_{1} \cdot Q_{1}(z)+\left(1-z^{-1}\right) Q_{2}(z) \\
D_{3}(z)=z^{-2}\left(\alpha_{1} H_{1}-F_{1}\right)\left(\alpha_{2} H_{2}-F_{2}\right) x(z) \\
\quad-z^{-2} F_{1}\left(\alpha_{2} H_{2}-F_{2}\right) Q_{1}(z) \\
+z^{-3}\left(F_{1}-\alpha_{1} H_{1}\right)\left(\alpha_{2} H_{2}-F_{2}\right) Q_{1}(z)-z^{-1} F_{2} Q_{2}(z) \\
+z^{-2}\left(F_{2}-\alpha_{2} H_{2}\right) Q_{2}(z)+\left(1-z^{-1}\right) Q_{3}(z)
\end{gathered}
$$

Here $\alpha_{i}$ and $k_{i}$ are the integration and quantizer gains respectively. The term $H_{i}$ is the digital gain factor per stage while $F_{i}$ is the feedback scaling factor. Given that $D_{\delta}(z)$ is the sum of the outputs of the three stages after appropriate delay processing, and if it is assumed that $H_{1}=1 / \alpha_{1}, H_{2}=1 / \alpha_{2}, F_{1}=1, F_{2}=1$, then $D_{0}(z)$ is given by

$$
D_{0}(z)=z^{-2} x(z)+\left(1-z^{-1}\right)^{3} Q_{3}(z)
$$

This result clearly indicates that third order quantization noise shaping is present in the output signal. The output also contains a representation of the input signal pro 


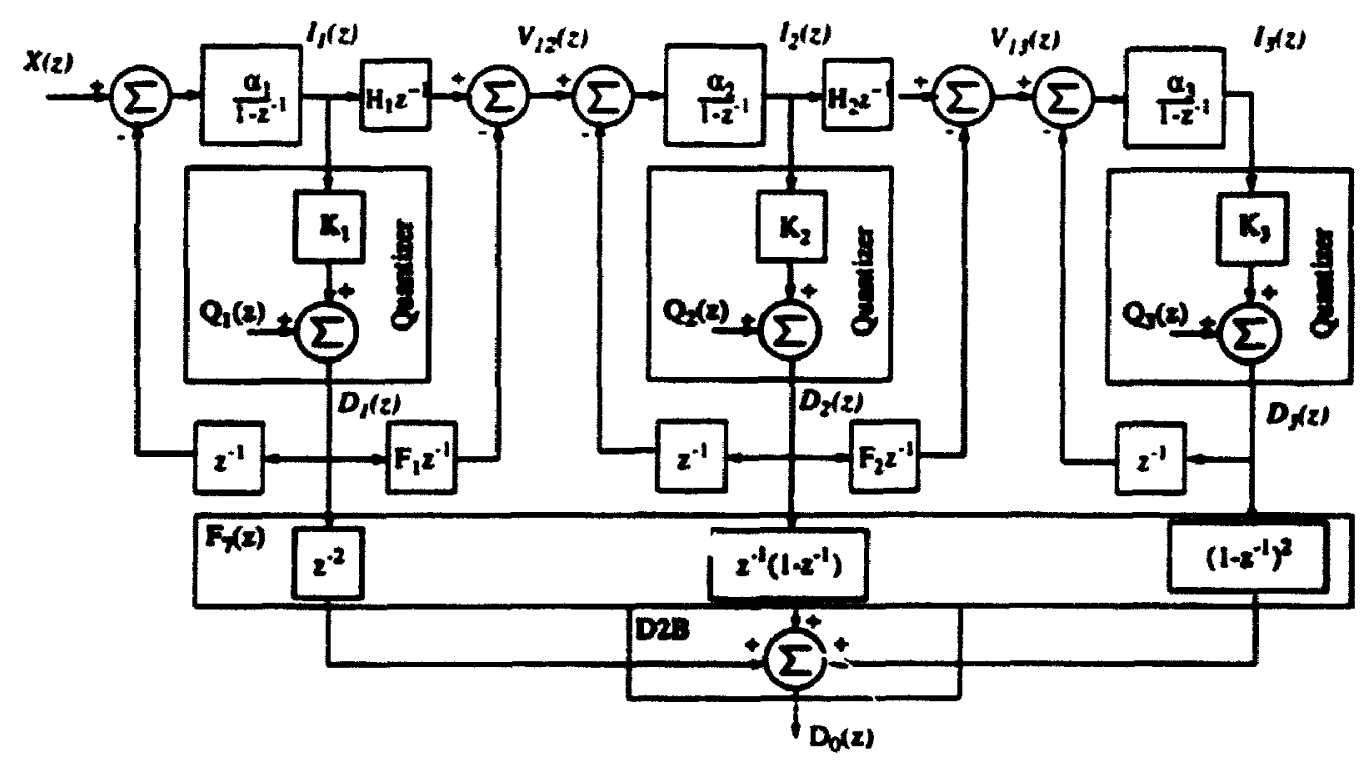

Figure 3.9 : A third order (MASH) $\Delta \Sigma$ modulator.

cessed by two delays.

In order to convert Longo's model from the sampled data model presented in Figure 3.9 to the digital accumulator model of Figure 3.8 it is necessary to assume that each of the $\Delta \Sigma$ stages of the modulator can be replaced by a $\mathrm{N}$-bit digital phase accumulator (DPA) and that $D_{l}(z), D_{2}(z)$ and $D_{3}(z)$ represent the carry outputs of each stage. Before proceeding to describe the third order structure used a companison between a first order $\Delta \Sigma$ modulator and a first order DPA is presented to verify the above assumption. These results are based on work by Riley and Copeland [RC92b]. Consider the diagram of Figure 3.10 (a). The difference equation for such a DPA is given by

$$
R_{n}=R_{(n-1)}+K_{n-1}-M \cdot \operatorname{carry}\left[R_{(n-1)}+K_{n-1}\right]
$$

Here $R_{n}$ is the remainder in the accumulator at the end of cycle $n$, and $K_{n}$ is the input value added to the accumulator during cycle $n$. The term $M$ represents the carry output value of the DPA and is given by $2^{k}$, where $k$ is the number of bits in the accumulator. The carry function is given by

$$
\text { carry }=R_{n-1}+K_{n-1}-\frac{M}{2} \operatorname{sign}\left(R_{n-1}+K_{n-1}-M\right)-\frac{M}{2}
$$


If $\left(K_{u}-M\right)$ is added to both sides of (3.33) then

$$
\left(R_{n}+K_{u}-M\right)=\left(R_{n-1}+K_{n-1}-M\right)+K_{n}-\frac{M}{2}-\frac{M}{2} \operatorname{siRn}\left(R_{n-1}+K_{n-1}-M\right)
$$

and if $x_{n}$ and $u_{n}$ are defined as

$$
\left(\begin{array}{c}
x_{n}=R_{n}+K_{n}-M \\
u_{n} \equiv K_{n}-\frac{M}{2}
\end{array}\right)
$$

then (3.33) can be rewritten in the form

$$
x_{n}=x_{n-1}+u_{n}-\frac{M}{2} \operatorname{sign}\left(x_{n-1}\right)
$$

This equation can be mapped to the block diagram of Figure 3.10 (b), which is that of a first order $\Delta \Sigma$ modulator.
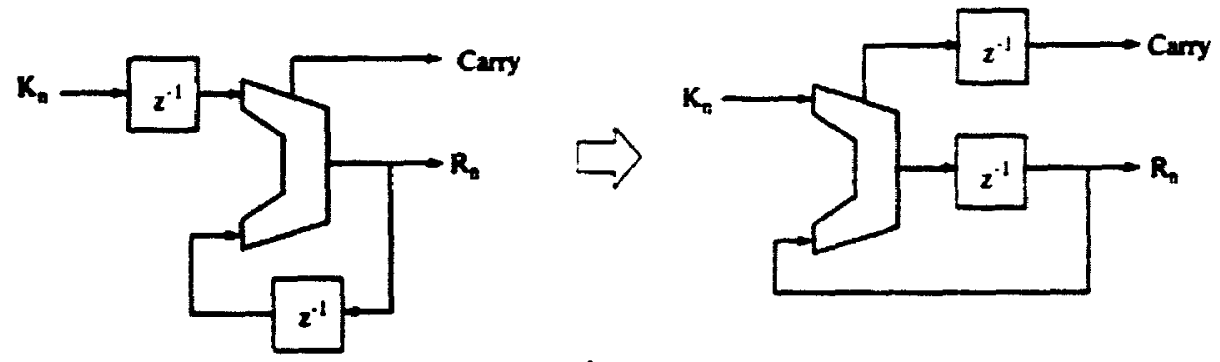

a)

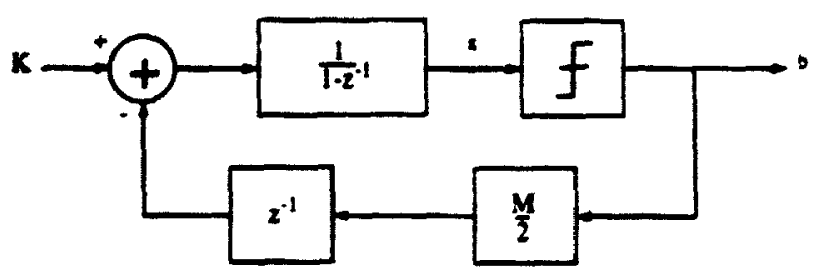

b)

Figure $3.10:$ a) a first order DPA b) a first order $\Delta \Sigma$ modulator

If the third order modulator of Figure 3.9 is now reconsidered, then each of the 
first order coders can be replaced by 25 bit DPAs. This provides a 25 bit third order DPA with carty signals defined as $D_{l}(z), D_{2}(z)$ and $D_{3}(z)$. The transformation process from $D_{l}(z), D_{2}(z)$ and $D_{3}(z)$ to $D_{0}(z)$ of Figure 3.9 is accomplished using the linear transformation function $F_{y}(z)$ and $D 2 B$, shown in Figures 3.11 and 3.12 .

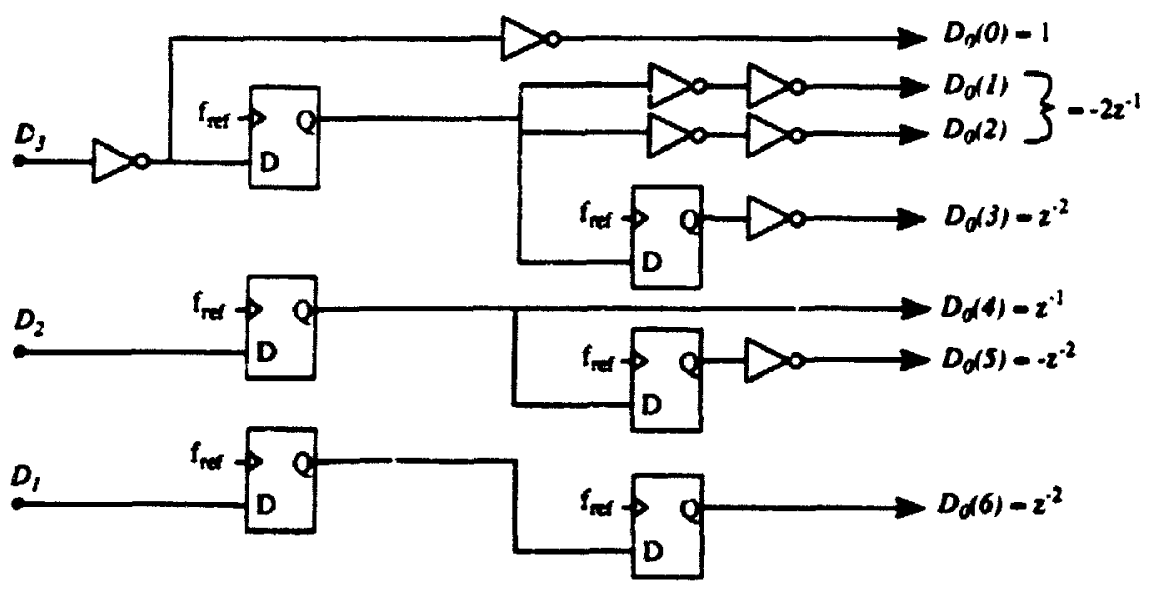

Figure $3.11: F_{7}(z)$, the third order quantization noise shaping function.

This function processes the 3 DPA carry outputs with pipelining, defined in Figure 3.9. to provide a binary output. The number of 1 's in the output of $D 2 B$ represent $D_{d}(z)$. The delay functions $c i$ Figure 3.9 can be expanded as follows.

$$
\begin{aligned}
& \left(1-z^{-1}\right)^{2}=1-2 z^{-1}+z^{-2} \\
& z^{-1}(1-z)^{-1}=z^{-1}-z^{-2}
\end{aligned}
$$

The output of $F_{7}(Z)$ is processed by function D2B as shown in Figure 3.8. This function inputs the 7 bits and counts the number of high bits on each input cycle and represents them as a binary two's complement 4 bit output. Figure 3.12 shows a block diagram of the function. The binary subtract 3 circuit is used to adjust the magnitude of the output bits of the D2B circuit such that this magnitude is centered around the input range of the concentrator circuit. 


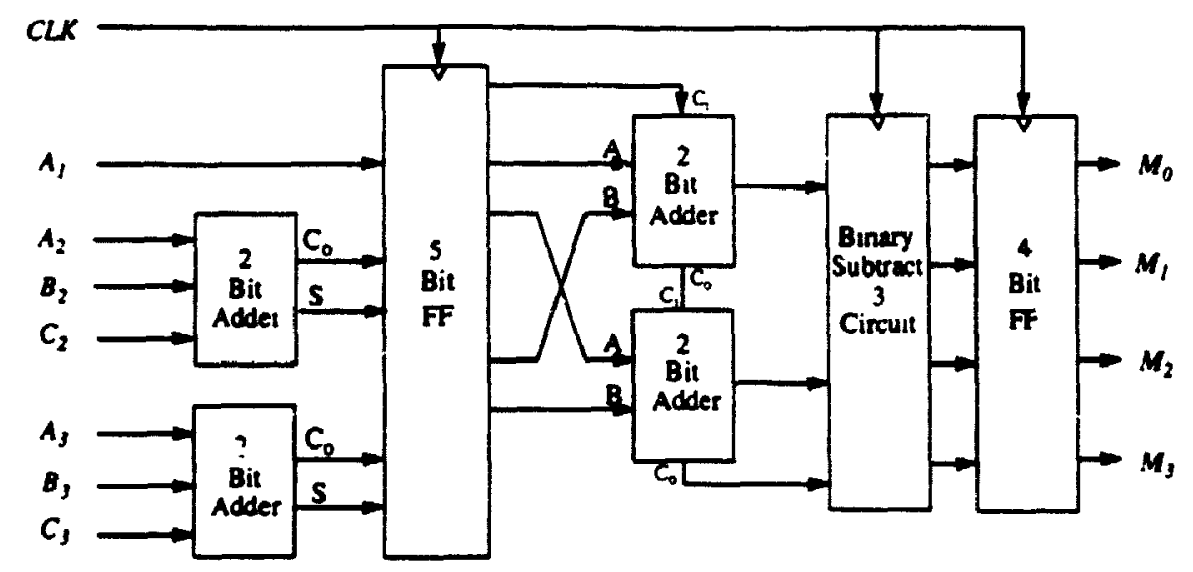

Figure 3.12 : Denury to binary (D2B) converter circuit

\subsubsection{A Third Order $\Delta \Sigma$ Concentrator}

The third order $\Delta \Sigma$ modulator presented in this section concentrates the 4 bit output of the D2B circuit of Figure 3.12 to a single bit bitstream, as indicated in Figure 3.8. This work, originally by Chao et. al. [CNL90], was empirically modified by Riley [RCK93] to develop the correct digital feedforward scaling factors. Figure 3.13 shows the modified block diagram of the modulator by Riley where the feedback factor $M$ is is implemented as a sign bit on the 4 bit adder.

The linearized transfer function for this modulator is given below, where $k$ is assigned a value of $1 / 4$ to fit the measured spectrum.

$$
H(z)=\frac{\left(1-z^{-1}\right)^{3}}{\left(1-z^{-1}\right)^{3}+k\left(1+4\left(1-z^{-1}\right)+8\left(1-z^{-1}\right)^{2}\right)}
$$

The theoretical power spectral density of this third order digital $\Delta \mathbf{\Sigma}$ modulator has been presented by both Chao and Riley and therefore the exercise is not repeated here.

The feedforward gain factors 1, 4, and 8 were implemented by bit shifts between the output bits of each respective accumulator and the feedforward input bits of the next adder stage. Each of these gain components differed by some power of 2 and 


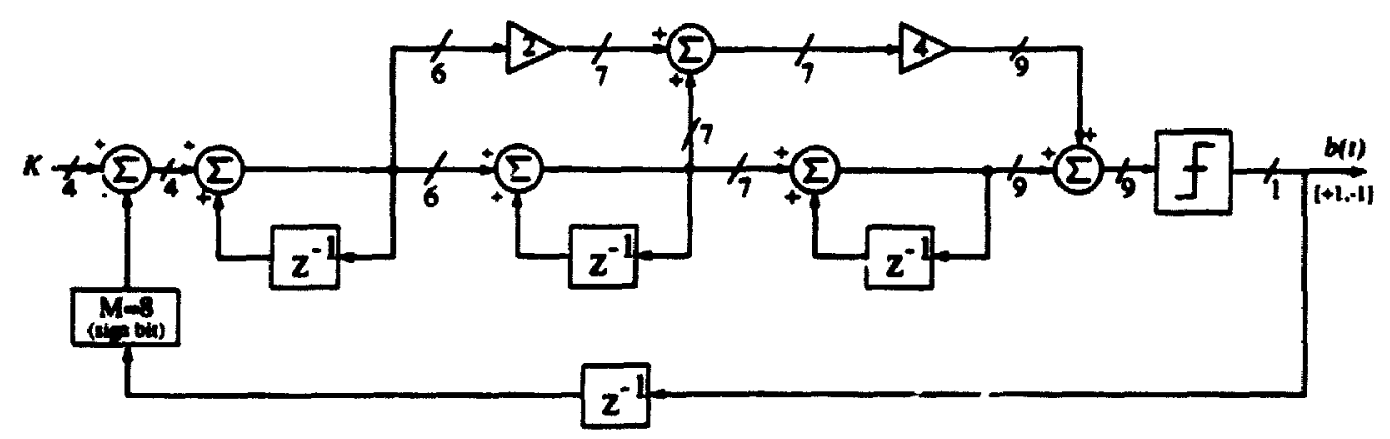

Figure 3.13 : A third order $\Delta \Sigma$ modulator with single bit bitstream output.

were therefore easily implemented in the digital modulator.

\subsubsection{Circuit Realization}

The full $\Delta \Sigma$ modulator of Figure 3.8 was implemented using a single field programmable gate array (FPGA) by Xilinx [Xi192]. The LCA 3090 was chosen because the device resources provided the number of configurable logic blocks (CLBs) required to accommodate the entire digital design. A printed circuit board was designed, fabricated, and then populated with a single LCA, an EPROM and other related hardware. This finished version was capable of providing third order quantization noise shaping to an operating frequency of $14 \mathrm{MHz}$.

Figure 3.14 shows the functional simulated power spectral density of the bistream output for the digital modulator circuit using a $10 \mathrm{MHz}$ reference frequency. This simulation was accomplished by generating a netlist of the schematic file and converti, 1 it to a format compatable with PC Silos.

A complete set of logic and wiring diagrams for the digital modulator can be found in Appendix B.

\subsubsection{Circuit Measurements}

The power spectral density of the quantization noise was measured by capturing the output bit stream of the modulator for a fixed digital (DC) input. The bitstream 


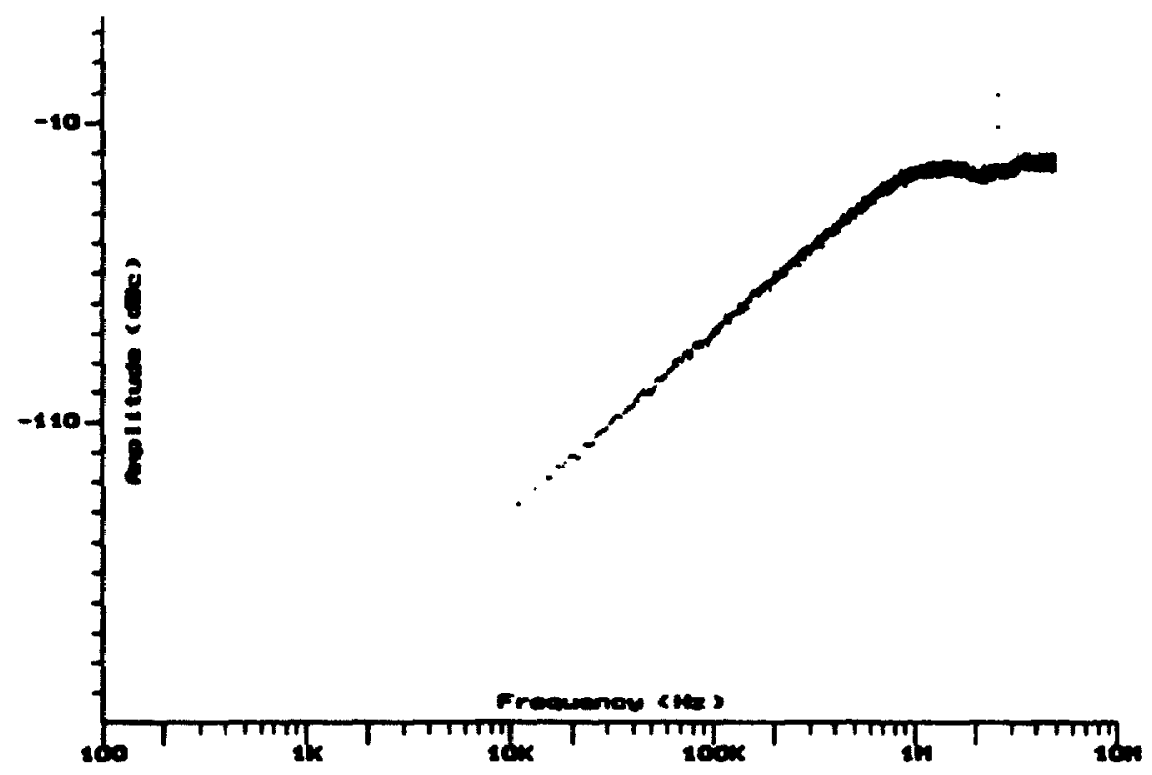

Figure 3.14: Simulated power spectral density of the complete third order digital $\Delta \mathbf{\Sigma}$ modulator

was captured using a computer card developed specifically for the purpose of grabbing bits. This bit grabber, synchronized to the $10 \mathrm{MHz}$ clock driving the $\Delta \Sigma$ modulator, captured the bits and stored them in a RAM buffer to be subsequently transferred $\omega$ a file in permanent memory on the computer. The bitstream file was then processed using software written by Riley [Ri2] to perform a DFT to generate and scale the power spectral density. Figure 3.15 shows the result of the measurement. The slope of the quantization noise is 3rd order which validates correct operation of the modulator. This result also compares favorably with the plot of the noise shaping of the power spectral density of the functional simulation shown in Figure 3.14.

\subsection{Frequency Synthesizer Measurement}

In this section, several fundamental measurements are presented that indicate the quality of the frequency synthesizer of Figure 3.1. These measurements include phase noise, transient response, and minimum frequency resolution. 


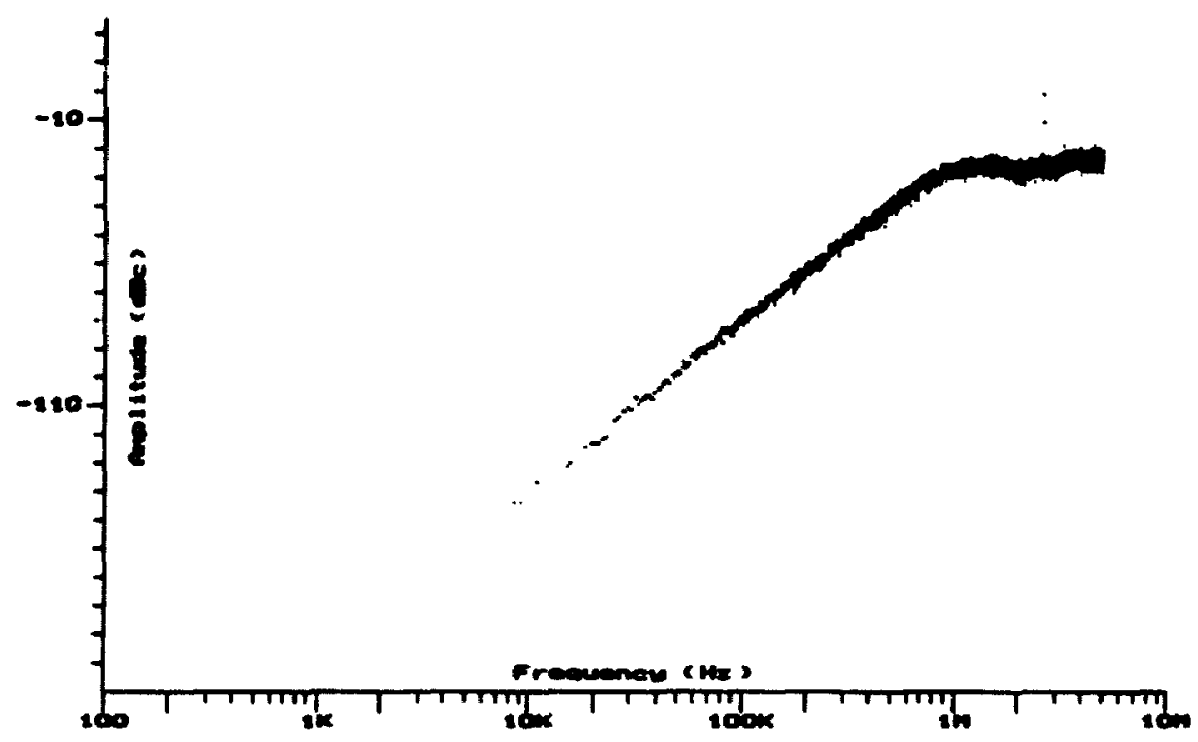

F.zure 3.15 : Measured power spectral density of the complete third order digital $\Delta \Sigma$ modulator

\subsubsection{Phase Noise Response}

Phase noise is a random short term frequency fluctuation which results generally from phase modulation of a carrier [HP1]. These short term instabilities in phase result in the creation of continuous noise sidebands which stretch out symmetrically from the carrier.

In radio receivers the level of the phase noise in the LO sidebands is translated directly to the IF sidebands. For a multichannel environment, sensitivity to a strong signal in an adjacent channel is dependent upon the level of phase noise at a full channel spacing from the carrier. In a frequency modulated system the presence of high levels of phase noise within the channel leads to reduced SNR for analog systems, and increased bit error rates for digital systems.

In a PLL frequency synthesizer the contour of the noise spectrum is dependant not just on the source reference but also on each of the components comprising the loop. Sorfleet [Sor91] presents a pertinent analysis of PLL synthesizer phase noise but limits the analysis to three noise sources, the reference frequency, VCO, and $\Delta \Sigma$ modulator. Poole [Po90] deals with synthesizer noise from a qualitive perspec 


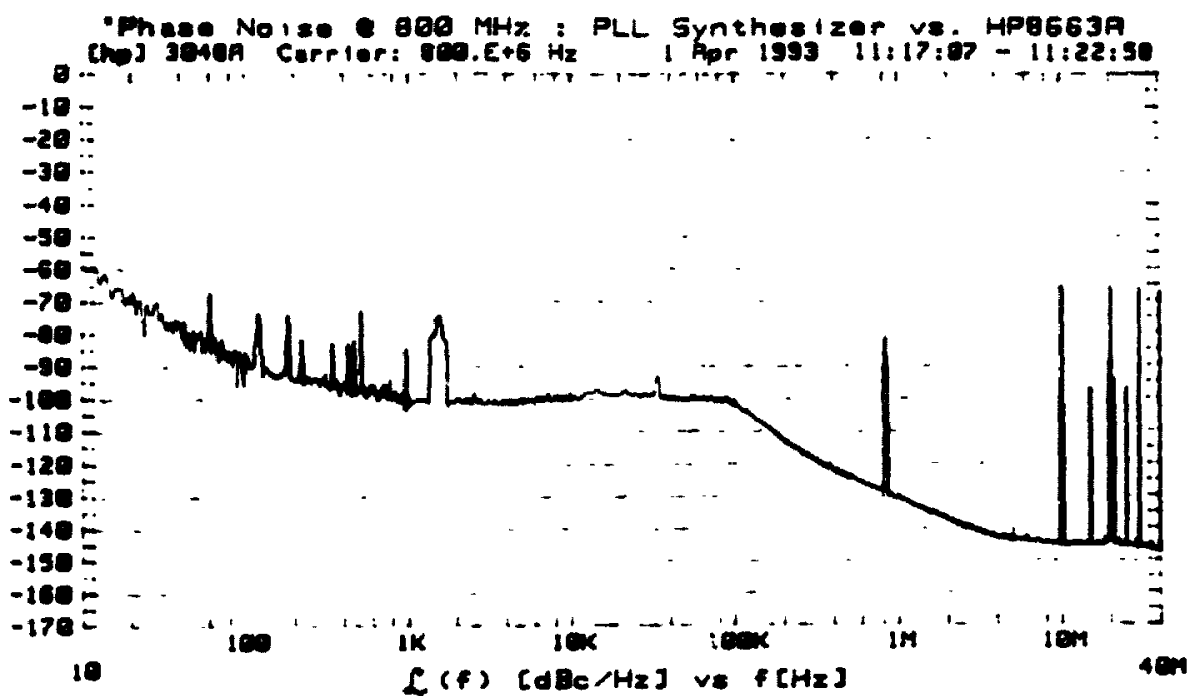

Figure 3.16: Single sideband phase noise response of an integer division frequency synthesizer at $800 \mathrm{MHz}$.

tive and provides a strong descriptive work on a more complete set of noise sources in a PLL frequency synthesizer. Sources of noise that can also contribute to the phase noise response are the phase-frequency detector, the frequency divider, and the loop response.

The multiplication factor of the divider $(\mathbf{N})$ degrades the phase noise response of the $\operatorname{loop}$ by adding $20 \log (\mathrm{N})[\mathrm{dB}]$ directly to the phase contour with respect to the VCO output. In efiect the noise of the frequency reference, the PFD, ana the loop filter are all multiplied by a factor of $N$.

The frequency reference allows some tuning of the phase contour, but acceptable levels of sounce jitter are dichted by the application. These levels can be manipulated by roplacement with a better quality component.

The digital phase detector and active loop filter are both capable of adding noise to the phase contour. The PFD becomes a noise generator when the loop is in lock and the detector is operating in the dead zone. In this sate the device outputs pulses that tend towards a zero width and therefore produce a detector gain that tends to zero. Noise can be produced with no change in the tune voltage if lange detector gain fluctuations occur around the zero gain state. This problem can be remedied by introducing a DC phase offect into one of the differential inputs of the 


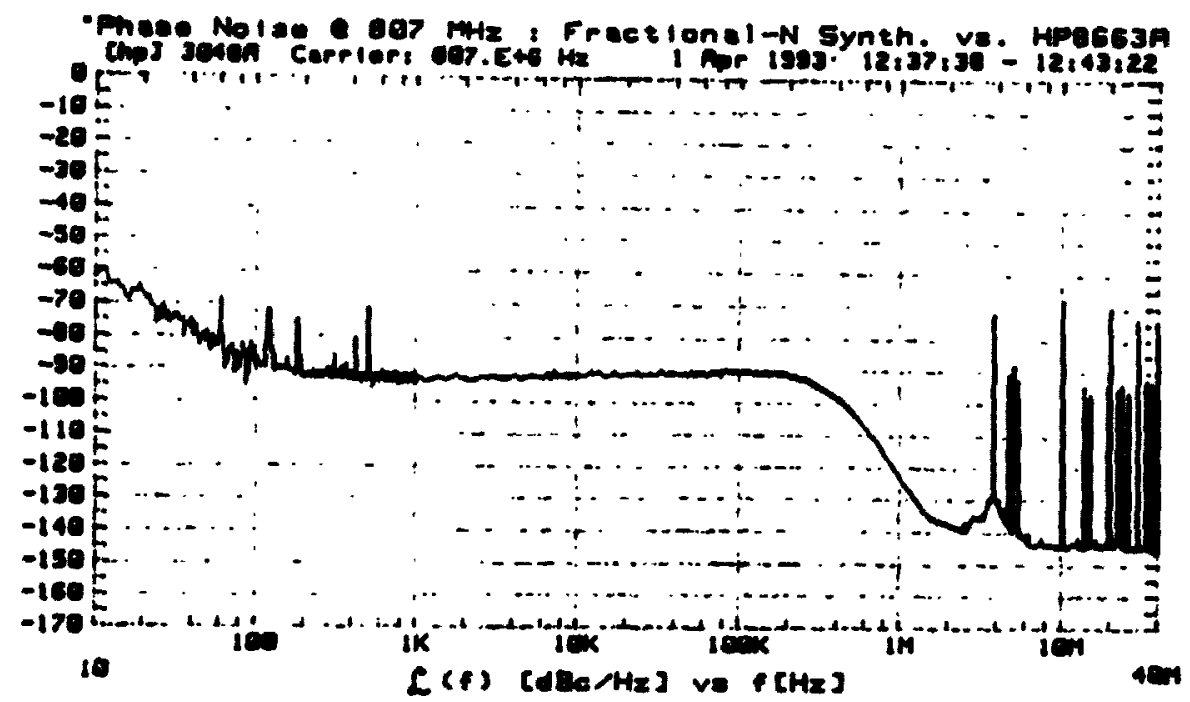

Figure 3.17 : Single sideband phase noise response of a fractional division frequency synthesizer at $810 \mathrm{MHz}$.

loop filter. This offect leads to a locked condition in the loop for a given input phase offect resulting in output pulses that can range from zero to 360 degrees width. Noice in a PFD can also realt from insuficicient power supply decoupling. Proper selection of copscitance scross the power supply terminals of the PFD component will hetp to reduce degandation in the noise performance of the PFD.

The loop response contributes to the phase contour in two was, bandwidth and loop stability. The loop bandwidth is a decign variable that must be chosen critically to ensure an optimum overall response between in-band appression and outof-band unsupresiad noise components (primarily VCO noise). Loop stability is ensured by decigning the loop filter such that the loop gain falls off at $20 \mathrm{~dB} /$ decade at the unity gain crosecover point.

VCO noise can arise from a number of different sources. Close in noise levels are a function of both the $Q$ of the component and flicker or $1 / f$ noise. Flicker noise can be reduced to some extent in bipolar circuits by using an emitter degeneration resistance to provide low frequency feedback. The $\mathrm{Q}$ of the VCO is a parameter that is directly ascociated with the tuning constant of the component. Inverently, VCOs with lage tuming range will have lower $Q$ values and therefore higher cloce in phase noise. At further out frequencies noise levels are more dependant upon noise figure (i.e. thermal noise), which indicates that noise levels will be depen- 
dant upon the power levels and type of device used in the construction of the VCO. What is presented here is the actual phase noise performance measurements of the synthesizer operating in both integer and fractional-N states. Figures 3.16 and 3.17 show the single sideband phase noise measurements respectively using Hewlett Packard's HP3048A phase noise measurement system [HP2] at $800 \mathrm{MHz}$ and $810 \mathrm{MHz}$.

\subsubsection{Transient Response}

The transient response of the synthesizer was measured in frequency for both integer and fractional frequency steps, as shown in Figures 3.18 and 3.19.

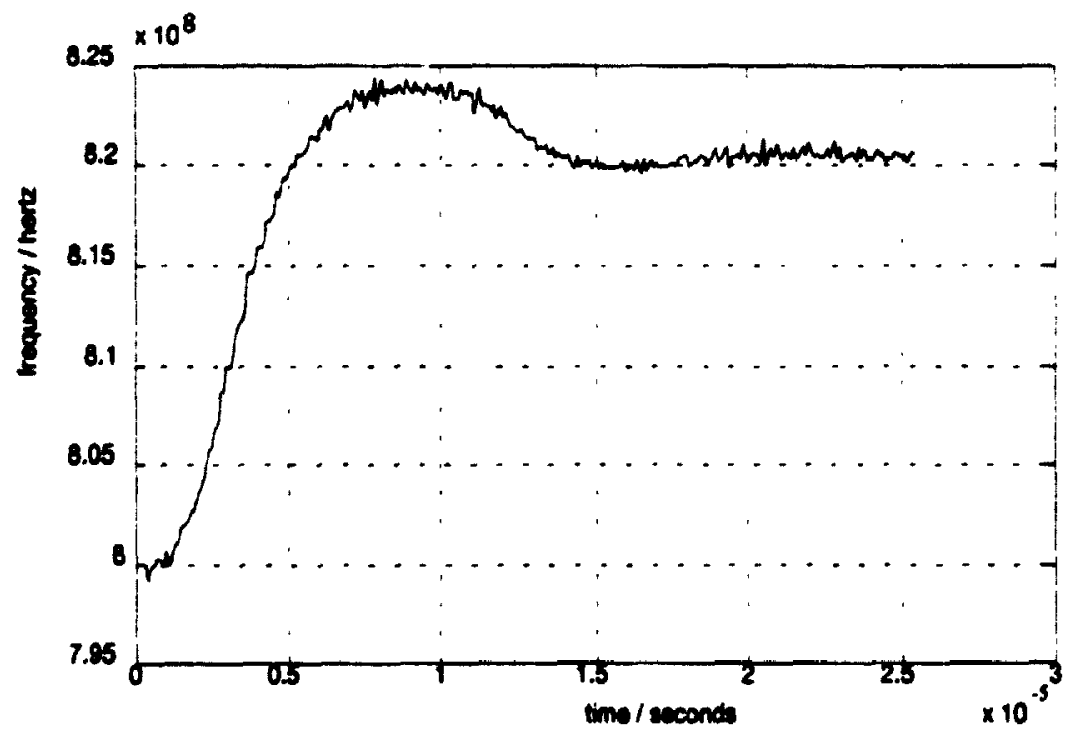

Figure 3.18: Transient response for an integer frequency step of $20 \mathrm{MHz}$.

The measurements, based on the use of the Hewlett Packard HP5372A Time Interval Analyzer [HP3], follow a procedure similar to that used by Sorfleet [Sor91]. The results are suitable for a rough indication of the loop dynamics but are limited by the measurement resolution of the HP5372A. Effects of the sampling noise by the HP5372A can be seen in Figures 3.18 and 3.19, and is measured to be approximately $300 \mathrm{KHz}$ peak to peak.

Intended uses of the synthesizer in either broadband coded FM (slithering) or fre 


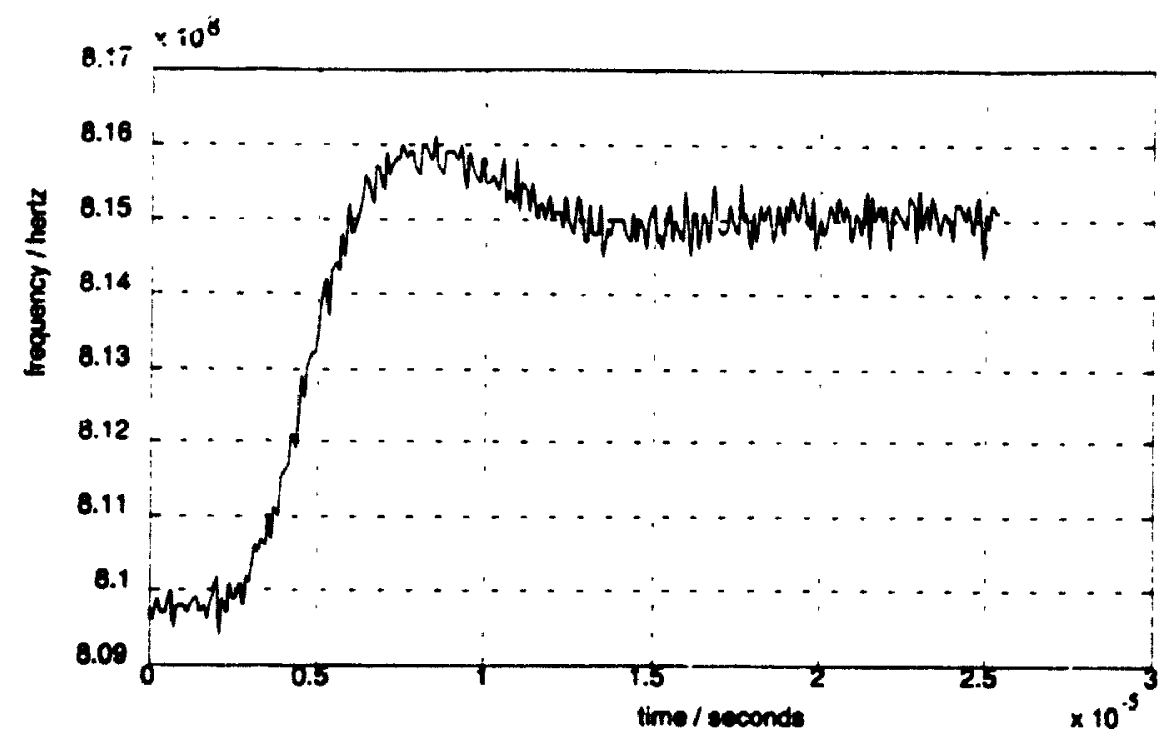

Figure 3.19 : Transient response for a fractional frequency step of $5 \mathrm{MHz}$.

quency hopping receivers dictates the need for both large (integer) and small (fractional-n) steps in the transient analysis. The integer step in frequency indicates the worst case scenario for loop response in a large hop in frequency. The fractional step in frequency will indicate the quality of the loop dynamics while operating with an average division ratio and a high level of quantization noise.

\subsubsection{Minimum Frequency Resolution}

The frequency synthesizer is controlled by a $\Delta \Sigma$ modulator with 28 bits on the input. The synthesizer output is capable of a maximum jump in frequency $\left(f_{\text {jump }}\right)$ equal to $\beta_{\max }$ times the PLL source reference, $f_{\text {ref }}$ when the prescaler is switched from $n$ to $\left(n+\beta_{\max }\right)$. This means the synthesizer will jump a total average frequency of $\left(10^{*} \beta_{\text {max }}\right)$ MHZ when the DMD (SP8782A) input is changed from $n$ tc $\left(n+\beta_{\max }\right)$. If $\beta_{\max }=1$ then the synthesizer will jump $10 \mathrm{MHz}$.

The minimum frequency resolution of the synthesizer is determined by measuring the difference in frequency when the input bits on the $\Delta \mathbf{\Sigma}$ modulator are toggled by a LSB. The minimurn frequency resolution can be determined using 


$$
f_{\text {res }}=\frac{f_{j u m p}}{2^{N}}
$$

Here $N$ is the number of input bits on the $\Delta \Sigma$ modulator. Equation (3.39) indicates an expected minimum frequency resolution of $0.0373 \mathrm{~Hz}$. The actual measured minimum resolution was found to be approximately $1 \mathrm{~Hz}$ for a movement of the $5^{\text {th }}$ significant bit. This correctly corresponds to the theoretical value of $0.0373^{*} 2^{5} \sim 1.19 \mathrm{~Hz}$. The frequency counter used for measuring this resolution was incapable of measuring less than $1 \mathrm{~Hz}$ for an input of $800 \mathrm{MHz}$. 


\section{Chapter 4}

\section{A $\Delta \Sigma$ Frequency Discriminator}

In digital FM radio receivers analog to digital conversion and data recovery can be achieved using a frequency discriminator. The discriminator presented in this chapter is a printed circuit boand version of the VLSI $\Delta \Sigma$ based frequency discriminator proposed by Beards and Copeland [BC92].

In general, a frequency discriminator provides an output signal proportional to the frequency deviation of the input [ZT85]. For an ideal discriminator, if the input signal is given by:

$$
x(t)=A_{c} \cos \left[\omega_{c} t+\phi(t)\right]
$$

The output will be

$$
y(t)=\frac{1}{2 \pi} K_{D} \frac{d \phi}{d t}
$$

For an FM system $\phi(t)$ is given by

$$
\phi(t)=\underset{0}{2 \pi f_{d}} m(\alpha) d \alpha
$$

This allows (4.2) to be rewritten as

$$
y(t)=K_{D} f_{d} m(t)
$$


Here $K_{D}$ is the discriminator constant (volts/hertz) and $y(t)$, the discriminator output. Under ideal operation the discriminator will have a linear frequency to voltage transfer function with an output magnitude of zero for $f=f c$.

The $\Delta \Sigma$ frequency discriminator described in this thesis, based on the work of Beards and Copeland [BC92], provides a digital output whose density of ones $\bar{B}$ is proportional to frequency deviations of the input signal. The density of ones is given by

$$
\bar{B}=-K_{D} f_{d}(t)
$$

For an $n /(n+1)$ DMD and a bipolar $\pm V_{D D} / 2$ output, then

$$
K_{D}=\frac{V_{D D} / 2}{f_{\text {ref }} / 2}=\frac{V_{D D}}{f_{\text {ref }}}
$$

and

$$
f_{d}(t)=f_{\text {in }}-\left(n+\frac{1}{2}\right) f_{\text {ref }}
$$

where $n f_{\text {ref }}<f_{\text {in }}<(n+1) f_{\text {ref. }}$.

Here $f_{i n}$ is the frequency modulated input and $n$ is tise lower modulii of the $n /(n+l)$ divide process.

For a digital bipolar system the discriminator output signal is given by

$$
V_{o}(t)=-K_{D} f_{d}(t)+V_{Q}(t)
$$

which is similar in form to the equation for an ideal discriminator. The negative sign indicates that an increase in frequency leads to a decrease in bit density. which can be arbitrarily removed by using $\bar{Q}$ on the output of the quantizer thas changing the definition of $K_{D}$ from a positive to a negative result. The component $V_{Q}(t)$ is the quantization ncise associated with the oversampling $\Delta \Sigma$ operation.

In the process of converting frequency deviation information into a digital bit- 
stream, quantization noise is produced that has a power spectral density characterized by a high pass transfer function. Post filtering must be appliet to remove this noise component and then decimation applied for reducion to baseband rate.

\subsection{Circuit Operation}

Consider the block diagram of Figure 4.1(a), a simplified model for a first order $\mathbf{\Delta \Sigma}$ discriminator.

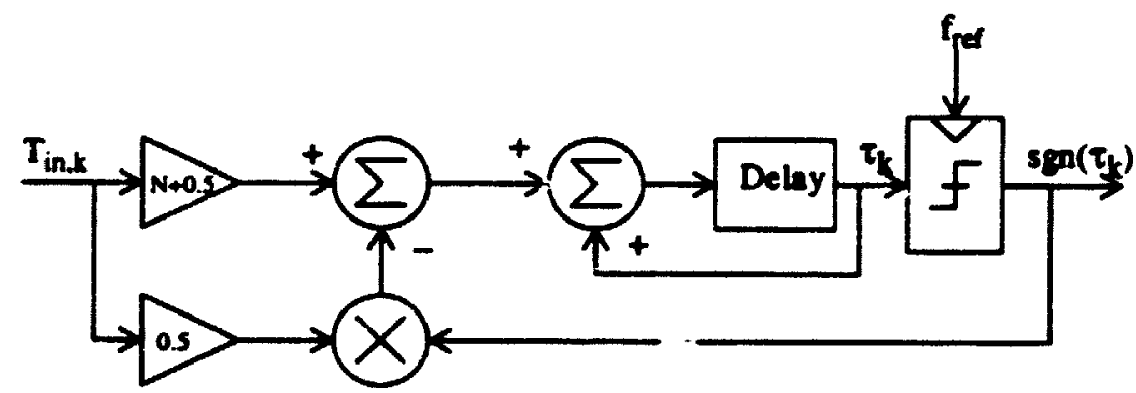

(a)

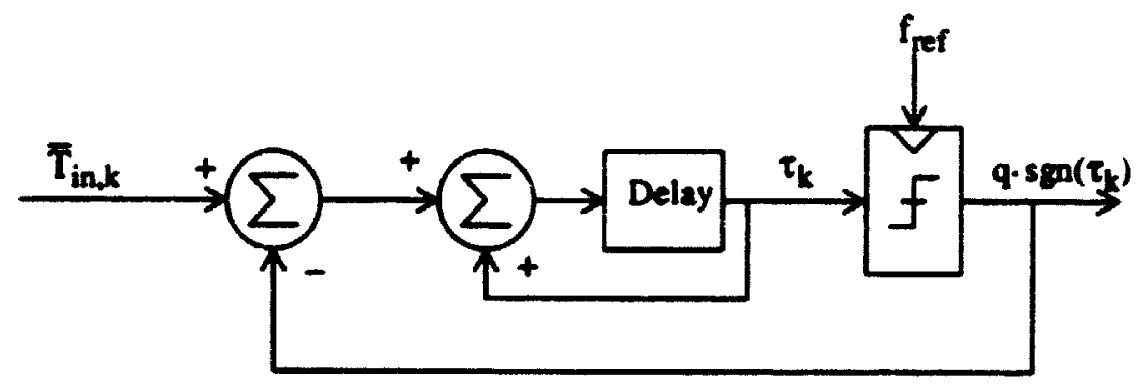

(b)

Figure 4.1 : (a) Equivalent block diagram of a first order $\Delta \mathbf{\Sigma}$ frequency discriminator; (b) modified block diagram of a first order $\Delta \Sigma$ frequency discriminator (reprinted from [BC92].)

The discriminator feedback factor is dependant upon the magnitude of the average frequency of the input signal. In the classic sense of $\Delta \mathbf{\Sigma}$ modulation this dependancy is not present, yet if narrow band modulation is assumed, then the small per- 
cent deviation $\delta$ on the input can be neglected leading to a definition for the coarse quantizion time edge deviation output of

$$
q=\frac{T_{i n, k}}{2}=\frac{T_{\text {carrier }}}{2}\left(1-\delta+\delta^{2}-\ldots\right) \equiv \frac{T_{\text {carrier }}}{2}
$$

For small deviations on $f_{\text {in }}$ the feedback signal $q$ is a time edge movement approximately equal to $1 / \mathrm{of}$ the carrier input period. The useful information contained in the input signal is the deviation due to the modulation of the carrier. An approximate model is shown in Figure 4.1 (b), where the input signal is redefined as

$$
\bar{T}_{i n, k}=\left[(n+\beta) T_{i n, k}-T_{r e f}\right]
$$

Here $n$ is an integer and $\beta$ a fraction between 0 and 1 , and the sum of the two defines the average division factor on $f_{i n}$. The valid range of operation for $(4.10)$ is $n T_{\text {in,k }}<T_{\text {ref }}<(n+1) T_{i n, k}$

Consider the simplified model of Figure 4.2, presented by Beards and Copeland [BC92], for the second order $\Delta \Sigma$ frequency discriminator.

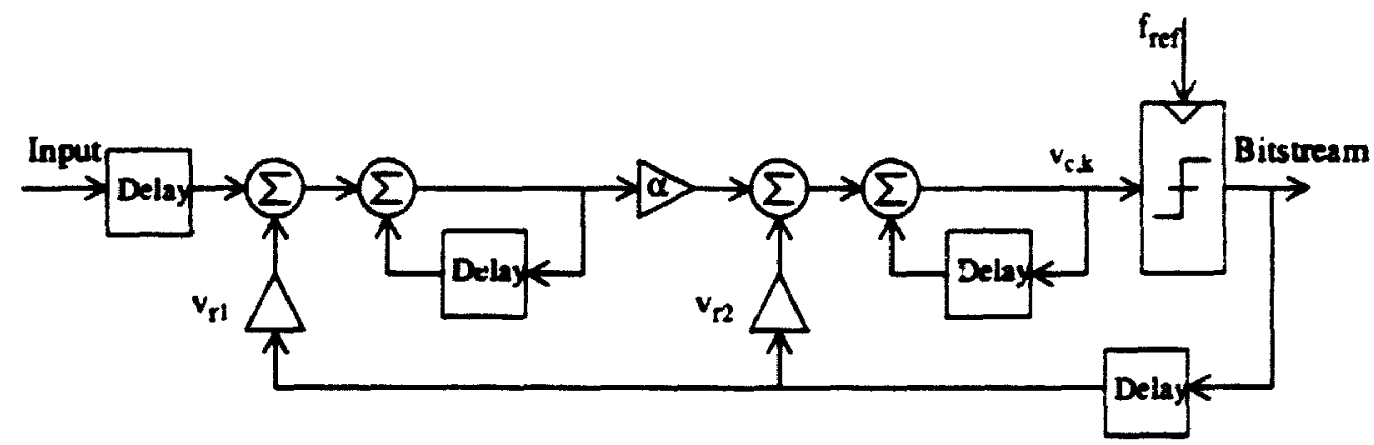

Figure 4.2: Model for the second order $\Delta \Sigma$ frequency discriminator (reprinted from [BC92].)

The difference equations defining the operation of this model are given by

$$
v_{c, k}=v_{c, k-1}+\alpha \tau_{k}-\operatorname{sgn}\left(v_{c, k-1}\right) v_{r 2}
$$




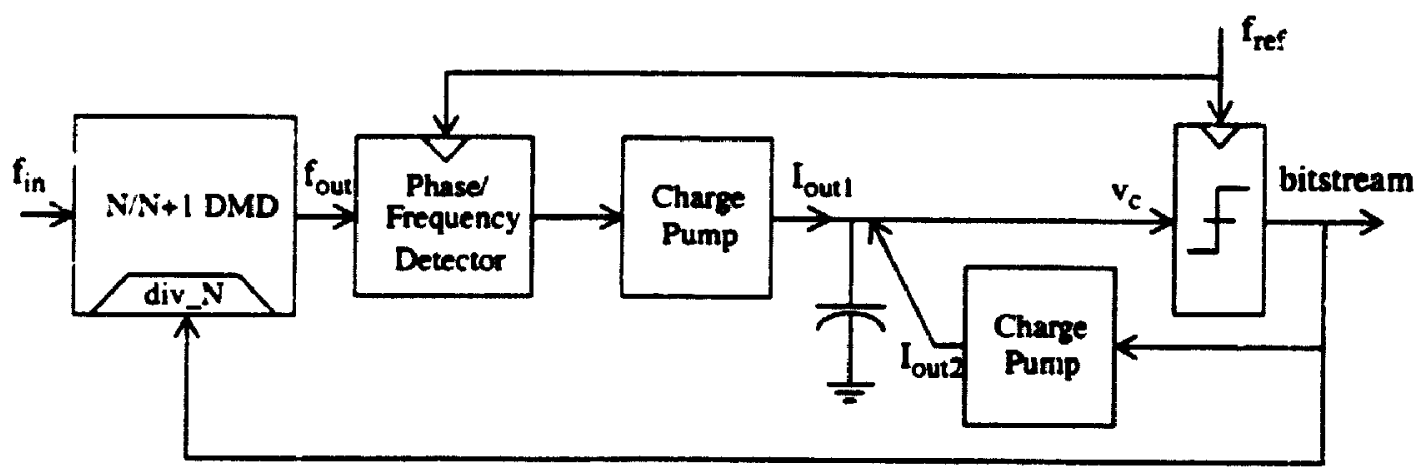

(a)

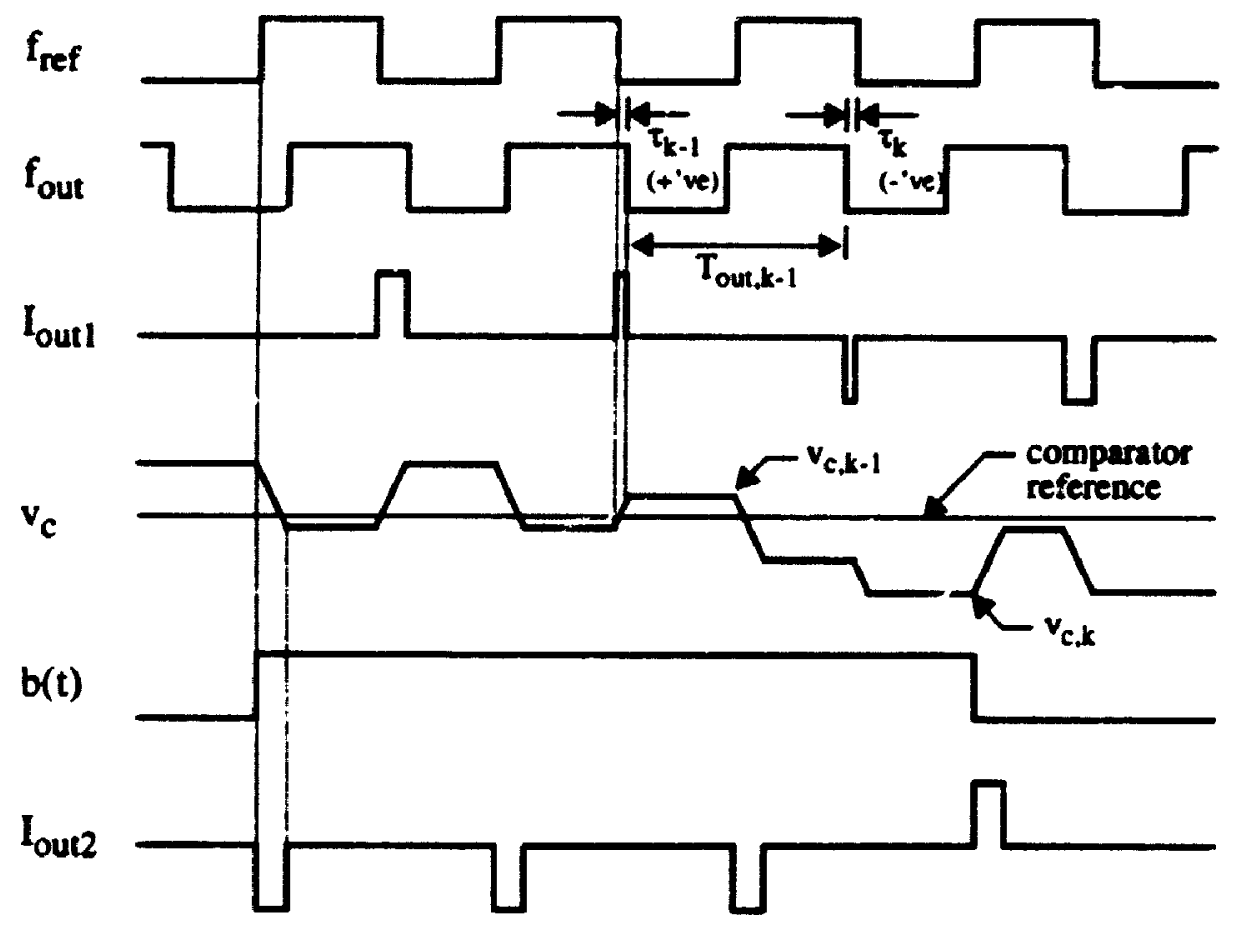

(b)

Figure 4.3 : a) Block diagram of the second order $\Delta \Sigma$ frequency discriminator, b) waveforms describing correct discriminator op:ration (reprinted from [BC92].)

$$
\tau_{k}=\tau_{k-1}+\left[(n+\beta) T_{i n, k-1}-T_{r e f}\right]-\operatorname{sgn}\left(v_{c, k-1}\right) \frac{T_{i n, k-1}}{2}
$$


Here $\alpha$ is the gain constant between the cascaded stages, and $v_{r l}$ and $v_{r 2}$ the gain factors on the magnitude of the feedback signals from the first and second stages respectively.

The $\Delta \Sigma$ frequency discriminator of Figure 4.3 (a) is the discriminator circuit implemented in this thesis. For this second order structure the waveforms of Figure 4.3(b) and the definitions of a first order structure provided in equations (4.9) and (4.10) allow for equation (4.12) to be rewritten as

$$
\tau_{k}=\tau_{k-1}+\bar{T}_{i n, k-1}-\operatorname{sgn}\left(v_{c, k-1}\right) \frac{T_{c a r r i e r}}{2}
$$

Equations (4.11) and (4.13) will remain valid as long as the oversampling rate $f_{\text {ref }}$ is much greater than the rate of deviation on the input signal (modulation rate) and as long as $n f_{\text {ref }}<f_{\text {in }}<(n+1) f_{\text {ref }}$.

From a qualitative perspective the circuit of Figure 4.3 (a) operates by comparing $f_{\text {our }}$, the divided down signal of $f_{\text {in }}$, with $f_{\text {ref }}$. Depending on whether $f_{\text {out }}$ is phase advanced or retarded with respect to $f_{\text {ref }}$, the output of the PFD will switch charge pump \#1 to either pump current on, or draw current off the integrating capacitor. On each cycle of $T_{\text {ref }}=\left(1 / f_{\text {ref }}\right)$ voltage $v_{c}$ is compared against a known reference level and the output is quantized.

This output is a one bit approximation ot ihe input phase difference $\bar{T}_{i n, k-1}$, and is used to conurol the switching of change pump $\$ 2$ to provide second order statistics, as well as toggle the modulus control of the DMD to reduce the phase error between $f_{\text {our }}$ and $f_{\text {ref }}$.

\subsection{Circuit Design}

For this thesis, the design of the $\Delta \boldsymbol{\Sigma}$ discriminator circuit was based upon the use of off-the-shelf components. In this section each component of the design shown in Figure 4.3 is investigated to present its underlying operation and how it must be implemented in order to ensure correct feedback sense and to ensure that an entire discriminator cycle occurs within a single period of the reference clock.

The design parameters for the discriminator were arbitrarily chosen to be 


$$
\begin{gathered}
f_{\text {in }}=100 \rightarrow 110 \mathrm{MHz} \\
\text { Deviation }=100 \mathrm{KHz} \\
\text { Modulation }=50 \mathrm{KHz} \\
f_{\text {ref }}=10 \mathrm{MHz}
\end{gathered}
$$

Here Deviation and Modulation are the frequency deviation and the modulation rate on $f_{\text {in }}$. The relationship between $f_{\text {ref }}$ and the $f_{\text {in }}$ range is dependant upon the value and ratio of modulii on the DMD. In this case $n$ equals 10 , and the DMD toggles between 10/11.

\subsubsection{The Dual Modulus Divider (DMD)}

The divider chosen for the discriminator was the Plessey SP8680B, a 600MHZ $\div 0 / 11$ tevice [Ples]. Internally the device is constructed of a 3 stage ECL Johnson counter with pulse swallowing techniques and a divide by 2 output stage to provide the $10 / 11$ divide capability.

It has a single ended input with an irternal 50K $\Omega$ pulldown resistor, and a differential output which requires external pulldown resistors to $\mathbf{- 5 . 2}$ volts.

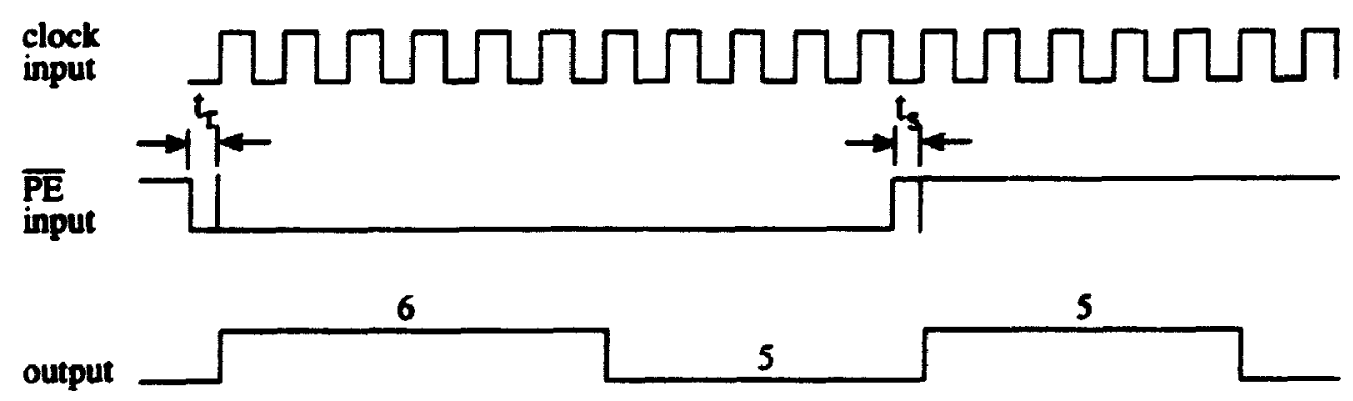

Figure 4.4 : Timing diagram for the Plessey SP8680, (renrinted from [PLES]).

From Figure 4.4 the set up time $t_{s}$ is defined as the minimum time that can elapse between a $L \rightarrow H$ transition of the control input and the next $L \rightarrow H$ input clock pulse 
transition to ensure that the $\div 10$ mode is obtained. As well, the release time $t_{r}$ is defined as the minimum time that can elapse between a $H \rightarrow L$ transition of the control input and the next $L \rightarrow H$ clock pulse transition to ensure that the $\div 11$ mode is obtained.

\subsubsection{The Phase Frequency Detector (PFD)}

The digital phase frequency detector chosen for the discriminator circuit was the Sony SPECL CXB111ZQ [Son89]. This is a fully differential ECL component that accepts data rates to $800 \mathrm{MHZ}$. The very short rise, fall and propagation times of this high speed device keep the width of the nonlinear distortion region (dead zone) reasonably small. In a closed loop structure such as the frequency discriminator, nonlinear distortion in the phase/voltage transfer function can lead to unwanted beat frequencies and noise translated into the baseband. The differential inputs on the device allow for suppression of common mode signals coupled to the input lines. As well the differential operation allows for suppression of power supply noise.

The operation of the PFD is summarized in the timing diagram of Figure 4.5.

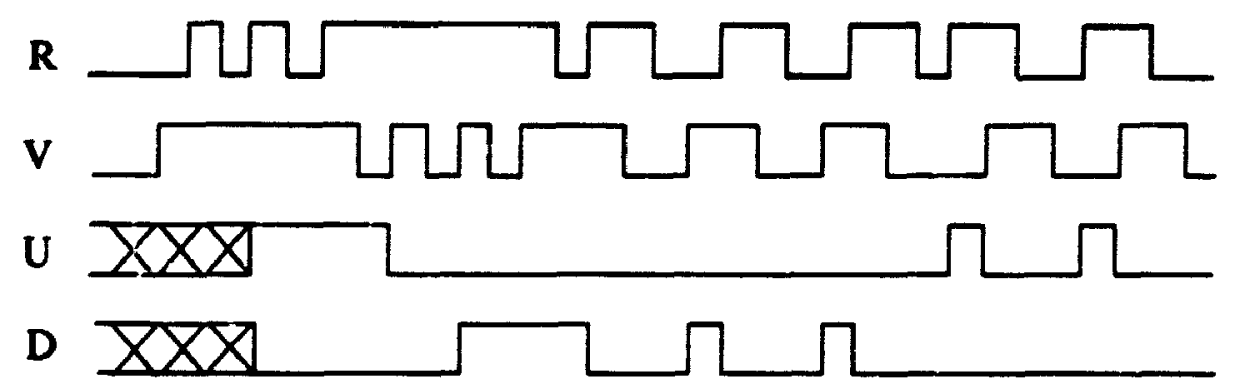

Figure 4.5 : Timing diagram for the Sony PFD, CXB1112Q (reprinted from [Son89]).

If the rising edge of the reference input $R$ leads the rising edge of the variable input $V$, then the up output $U$ goes high on the rising edge $R$ and resets on the next rising edge of $V$. As long as $R$ leads $V$ the pulse width of $U$ will vary linearly with the phase difference of $R$ and $V$, but the down output $D$ will remain low. Similarly if the rising edge of $V$ leads the rising edge of $R$, then on the rising edge of $V$ the output $D$ goes high and then resets on the next rising edge of $R$. As long as $V$ leads $R$ 
then the pulse width of $D$ will vary linearly with the phase difference of $V$ and $R$, but the up output $U$ remains low. If the timing edges of both $V$ and $R$ are synchronized then both $D$ and $U$ remain low producing a neutral state output. This description provides a way for understanding how the charge pump will work.

\subsubsection{Charge Pump Circuit Operation}

The charge pump circuit of the frequency discriminator was designed to convert the analog time edge information on the PFD differential outputs to analog voltage levels by switching quantized packets of current onto integrating capacitors. This provided the second order integration for the discriminator. The feedforward packets of current are a direct function of the pulse widths of the UID signals and therefore linearly related to the time edge difference between the modulation and the reference signals on the input of the PFD.

The full charge pump circuit consists of a set of differential feedforward current switches, a set of differential feedback current switches, and a common mode feedback component to suppress common mode drift on the differential integrating capacitors.

The block diagram of Figure 4.6 indicates the simplified operation of a set of differential current switches.

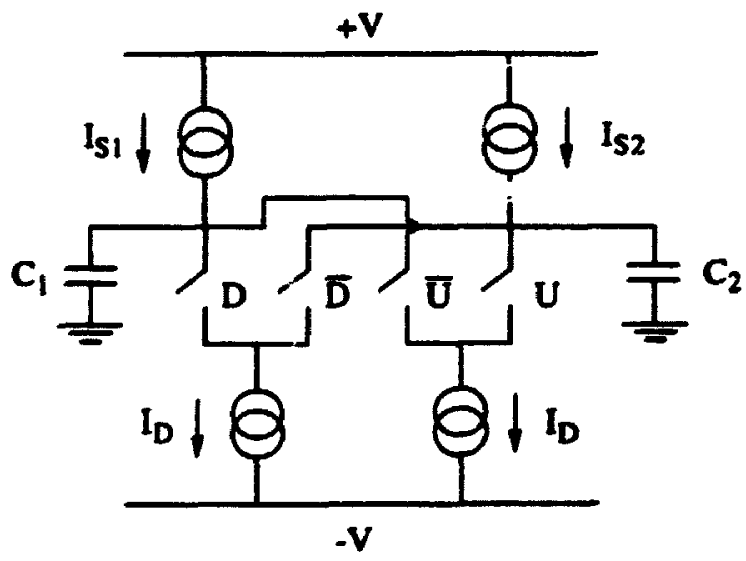

Figure 4.6 : Simplified differential charge pump 
Let switches $D, \bar{D}, U$ and $\bar{U}$ represent the output signals of the PFD, and let the source current $I_{S}$ equal the drain current $I_{D}$.

Assuming $R$ leads $V$, then $U$ is high for a period $\tau_{l}$. During $\tau_{l}$, switches $U$ and $\bar{D}$ are closed drawing a total current of $2 I_{D}$ drawn to $-V$. The source current $I_{S Z}$ supplies $50 \%$ of this amount, the other $50 \%$ is drawn from capacitor $C_{2}$ discharging it by amount $\tau_{l} l_{D}$. At the same time, with switches $\bar{U}$ and $D$ open $C_{l}$ is charging by amount $\tau_{l} I_{S l}=\tau_{l} I_{D}$. This is a complementary differential movement on capacitors $C_{1}$ and $C_{2}$. Conversely, if $V$ leads $R$, then $D$ is high for a period $\tau_{2}$. During $\tau_{2}$ switches $\bar{U}$ and $D$ are closed while $U$ and $\bar{D}$ are open. In this state $C_{2}$ is discharging by amount $\tau_{2} I_{D}$ while $C_{2}$ is charging by amount $\tau_{2} I_{S_{2}}=\tau_{2} I_{D}$. Again this is a complementary differential movement on $C_{1}$ and $C_{2}$.

For $R$ and $V$ synchronized, then $U$ and $D$ are both open while $\bar{U}$ and $\bar{D}$ are closed. and currents $I_{S l}$ and $I_{S 2}$ move from $+V$ to $-V$ without any charging or discharging of capacitors $C_{l}$ and $C_{2}$. This is the neutral state.

An obvious problem with a non-ideai circuit is that $I_{S}$ most protably will not equal $I_{D}$. If $I_{S}>I_{D}$, then for long periods of the neutral state, capacitors $C_{l}$ and $C_{2}$ can gradually charge up to $+V$. Alternatively, if $I_{S}<l_{D}$ then capacitors $C_{l}$ and $C_{2}$ can gradually discharge to $-V$ through the same mechanism. Therefore this type of charge pump can require a feedback network to suppress common mode drifts to either $+V$ or $-V$. Figure 4.7 shows the charge pump circuit with the simplified common mode feedback component included [Bea90].

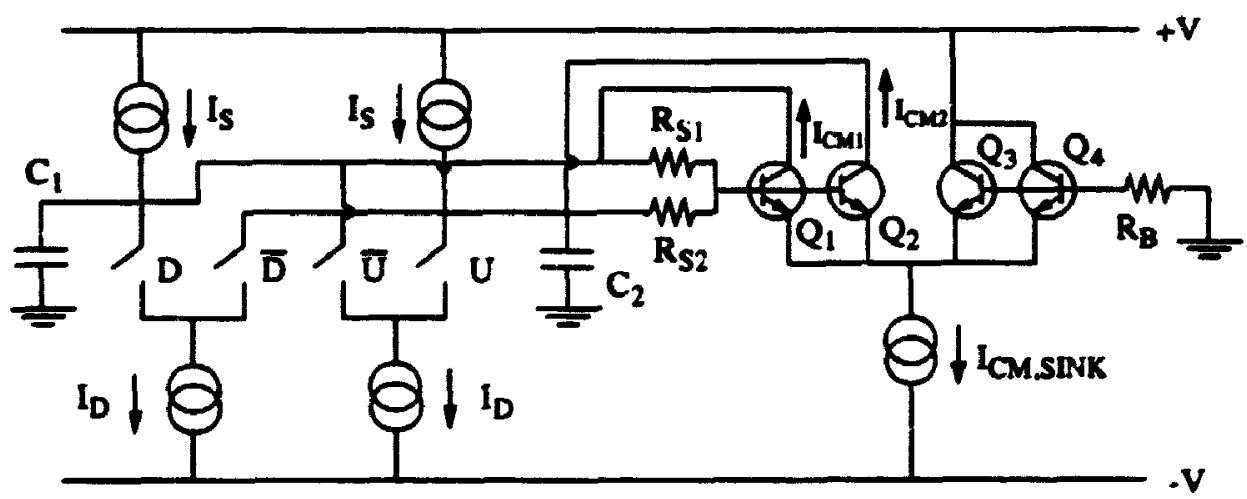

Figure 4.7 : Charge pump model with simplified common-mode feedback.

From Figure 4.7 current $I_{S}$ now equals $I_{D}+I_{c m}$, where $I_{c m, s i n k}$ equals 4 times $I_{c m}$. The doubly differential feedback circuit works by sensing common mode voltage 
levels on $C_{1}$ and $C_{2}$ through sense resistors $R_{S 1}$ and $R_{S 2}$. If $C_{1}$ and $C_{2}$ are charging differentially then the voltage level seen on the base of $Q_{1}$ and $Q_{2}$ remains unchanged and currents $I_{\mathrm{cm} I}$ and $I_{\mathrm{cm} 2}$ remain static. If the charge on capacitors $C_{l}$ and $C_{2}$ begin to drift up, resistors $R_{S I}$ and $R_{S 2}$ develop a rising voltage on the base of $Q_{1}$ and $Q_{2}$. This level is compared to the reference level across $R_{b}$, where the difference can lead to $Q_{1}$ and $Q_{2}$ turning on more deeply drawing more current off of $C_{l}$ and $C_{2}$ bringing their levels down to the reference level set by the base of $Q_{3}$ and $Q_{4}$. Alternatively, if $C_{1}$ and $C_{2}$ drift toward $-V$ then by the same negative feedback mechanism $Q_{l}$ and $Q_{2}$ can turn on less deeply drawing less current off $C_{l}$ and $C_{2}$ allowing them to charge up to the reference level developed across $\boldsymbol{R}_{b}$.

\subsubsection{Charge Pump Circuit Design}

The design of the charge pump circuit is based upon some arbitrary choices about initial design parameters. From Figure 4.3 (a) the full voltage (i.e. dynamic) range on the integrating capacitor was set to

$$
\Delta V_{T O T}=\frac{10 \times \Delta Q}{C}
$$

The discrete charge component $\Delta Q$ is based upon a step size of $1 /$ the input period $T_{i n}$, and is given by

$$
\Delta Q=\frac{T_{\text {in }}}{2} \times I_{1}
$$

The discrete charge component contributed by the feedback current $I_{2}$ is a function of $T_{\text {ref }}$ and is given by

$$
\Delta Q_{2}=\frac{T_{r e f}}{4} \times I_{2}
$$

From Figure 4.3 (b) the effect of feedback current $I_{2}$ on the comparator voltage $V_{c}$ is a fixed voltage step of 


$$
\Delta V_{C 2}=\frac{\mp \Delta Q_{2}}{C}
$$

for each cycle of $f_{\text {ref }}$. The polarity of the charge is dependant upon the instantaneous magnitude of $b(t)$.

The discrete feedforward charge component contributed by $I_{l}$ is dependant upon the difference between the falling edges of $f_{\text {ref }}$ and $f_{\text {out }}$. Empirical results [Ril] indicate that this difference $\tau_{k}$ is unlikely to exceed ten times the same polarity step size of $T_{I N} / 2$. Typically $\tau_{k}$ will change polarity every 3 to 4 cycles due to the nature of the coarse quantization involved. The feedforward charge component is given by

$$
\Delta Q_{1}=\tau_{k} \times I_{1}
$$

with the effective change on $V_{C}$ due to $I_{l}$ being

$$
\Delta V_{1}=\frac{\mp \Delta Q_{1}}{C}
$$

The sign on $\Delta V_{C l}$ is dependant upon whether $f_{\text {ous }}$ is phase advanced (-ve) or phase retarded (+ve) with respect to $f_{\text {ref }}$

The models for analog $\Delta \boldsymbol{\Sigma}$ modulators typically symbolize the feedforward and feedback scaling factors by $\alpha_{1}$ and $\alpha$, respectively. In the charge pump circuit these scaling factors are directly a function of the current levels $I_{l}$ and $I_{2}$.

The relationship $\alpha_{I}=\alpha_{2}$ can be paralleled in the charge pump by setting $\Delta Q_{I}=$ $\Delta Q_{2}$. This relationship has been shown to provide stable operation [CT1] and can be expressed as

$$
\frac{I_{1} \times T_{i n} / 2}{C}=\frac{I_{2} \times T_{\text {ref }} / 4}{C}
$$

Through the operation of the DMD, it is also known that 


$$
T_{\text {in }}=\frac{T_{\text {ref }}}{n+0.5}
$$

for a midrange fixed $f_{i}$ equency input. Substituting (4.21) into (4.20) and rearranging yields

$$
\frac{I_{2}}{I_{1}}=\frac{4}{2(n+0.5)}
$$

The application of the dynamic range $D R=I O \Delta Q$ provides an additional design equation of

$$
\frac{I_{2} \times T_{r e f} / 4}{C}=\frac{D R / 2}{10}
$$

If Figure 4.8, a n. Jre complete model for the charge pump circuit, is considered then the above equations can be used to determine the required circuit elements.

Due to the large number of variahles in the circuit design, initially some variables had to be chosen arbitrarily. Capacitors $C_{l}$ and $C_{2}$ were set to be $15 p f$. Any value less than this would be within an order of magnitude of the parasitic capacitances of the charge pump transistors. The DMD chosen was to be a $\div 10 / 11$ component. This allowed $f_{\text {in }}$ to range from $100 \rightarrow 110 \mathrm{MHZ}$ for a $10 \mathrm{MHZ}$ reference frequency. The dynamic range on $C_{l}$ and $C_{2}$ was set to 2 volts to match the full input swing on the latched comparator/quantizer. This allowed a \pm 1 volt swing around the common mode bias point of -0.7 volts. From (4.23) $I_{2}$ was calculated to be $60 \mu A$, and substituting into $(4.22) I_{l}$ was found to be $315 \mu \mathrm{A}$.

The common mode feedback circuit was designed interactively using Spice [Spi] simulation techniques based upon the initial assumption that

$$
\frac{g m}{2 \pi C} \cong U G B W
$$

where 


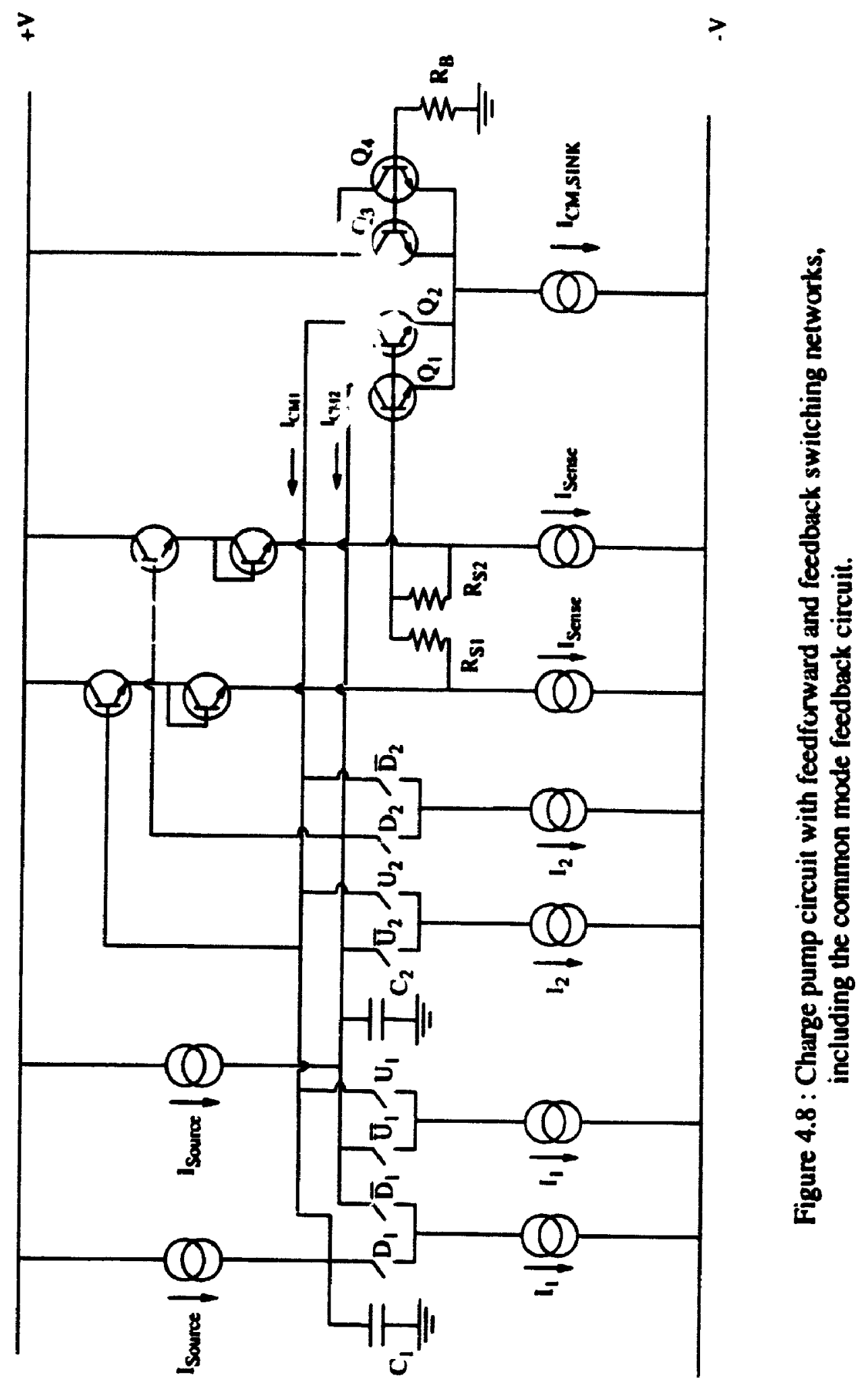




$$
g m=\frac{l_{c, c m}}{V_{T}}
$$

From the transconductance, the common mode current is given by

$$
I_{c, \mathrm{~cm}}=V_{T} \times 2 \pi C \times U G B W
$$

For the transistors used (2N3904), with a unity gain bandwidth of $\cong 300 \mathrm{MHZ}$ and a $V_{T}=k T / q=26 \mathrm{mV}$ (assuming a temperature of $300 \mathrm{~K}$ ), a common mode current of $735 \mathrm{~mA}$ was determined. Yet from (4.25) the gain of the common mode feedback circuit is directly related to $I_{c, c m}$. To ensure a suitable amount of common mode suppression $I_{c, c m}$ was arbitrarily set $104 \mathrm{~mA}$ to raise the gain as much as possible. Open loop SPICE simulations of the charge pump circuit indicated a UGBW of 26MHZ and a phase margin of 47.2 degrees. At $10 \mathrm{MHZ}$, the frequency of operation for the comparator, the gain was $14.3 \mathrm{~dB}$. With ! , cm $_{\text {set }}$ to $4 \mathrm{~mA}$, the current sink for the common mode circuit $I_{c, s i n k}$ becomes $16 \mathrm{~mA}$.

Knowing the values of all other currents in the circuit, the single ended source currents were calculated using

$$
I_{\text {source }}=I_{1}+I_{2}+I_{c, c m}
$$

From (4.27) $I_{\text {source }}$ was determined to be $4.375 \mathrm{~mA}$. Figure 4.9 (a) shows a diagram of the circuit used for the generation of the source currents. The 2000 potentiometer used in the source current circuit allowed the introduction of a small DC offset current between each of the source currents. This in tum allowed the tuning of a fixed offset voltage between the levels on the differential charging capacitors. The offset provided the capability of steering the operation of the PFD away from the dead zone.

Figure 4.9 (b) shows the circuit diagram of a Widlar current source used for the realizision of currents $I_{1}, I_{2}, I_{\text {sense, }}$ and $I_{c m_{1} \text { sint }}$

For the sensing node of Figure 4.8 the sink current $I_{\text {sense }}$ was arbitrarily set to $4 \mathrm{~mA}$. For the same circuit an emitter follower and diode connected transistor were used to isolate and level shift each of the sense node resistors $R_{S I}$ and $R_{S 2}$ from the inte 


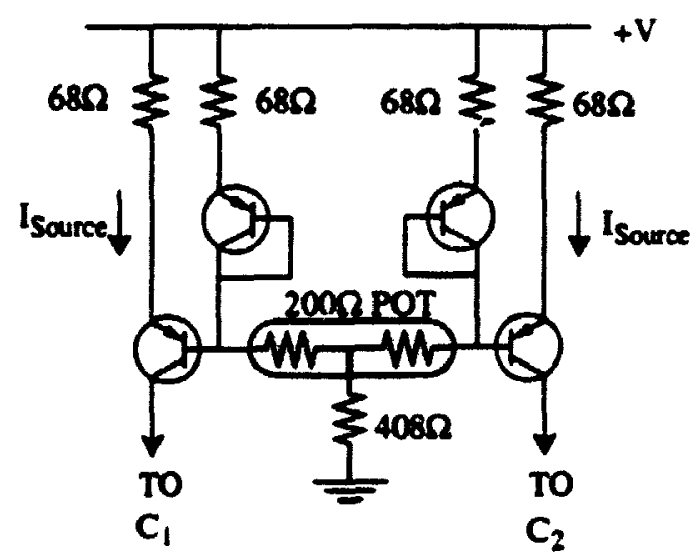

(a)

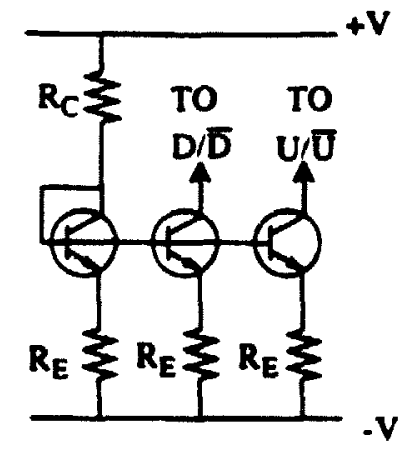

(b)

Figure 4.9 : a) Source current circuit; b) current sink circuit.

grating capacitors.

A complete circuit diagram of the charge pump of Figure 4.8 , including calculated values for bias components, can be seen in Appendix C.

\subsubsection{The Comparator/Quantizer and Interface Circuit}

The quantizer used in the discriminator is a high speed ECL pre-production device (MC10E1651) provided by Motorola [Mot3]. The I.C. consists of a pair of differential amplifiers with output latches. When the latch clock is high $Q_{n+1}$ reflects the instantaneous result of the input comparison as an ECL output level. When the latch clock goes low the instantaneous result of the input comparison is latched as an output ECL level. In onder to realize a complete flip flop action, the dual latched comparators were connected in a master-slave configuration.

Figure 4.10 shows a block diagram of the full discriminator circuit. This diagram shows an ECL PAL used to generate both reference and comparator clocks for the circuit.

The ECL PAL uses a $\div 4$ Johnson counter for the reference $(R, \bar{R})$ and comparator (CLO) clocks. The PAL is also used to generate a set of differential signals $U_{2}, \bar{U}_{2}$, $D_{2}$ and $\bar{D}_{2}$ that drive the feedback current switches of the charge pump. As shown 


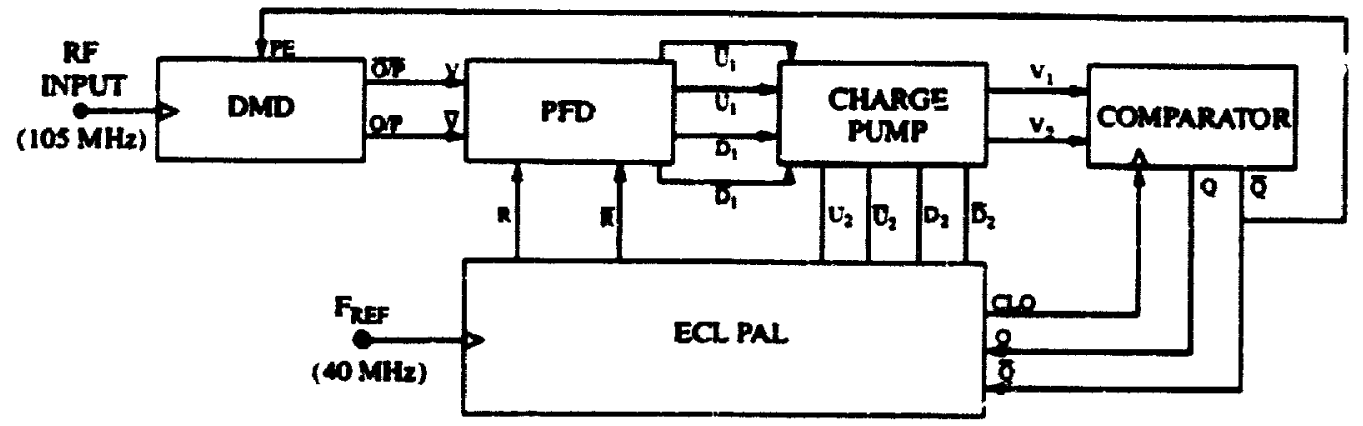

Figure 4.10: Block diagram of the complete discriminator.

in Figure 4.3 (b) current $/ 2$ has a fixed period and duty cycle. In the design parameters the duty cycle was set at $25 \%$ or a pulse width of $T_{\text {ref }} / 4$. For this reason a $40 \mathrm{MHZ}$ reference clock was used to generate the $10 \mathrm{MHZ}$ signals needed for the reference on the PFD. The two orthogonal outputs of the Johnson counter allowed

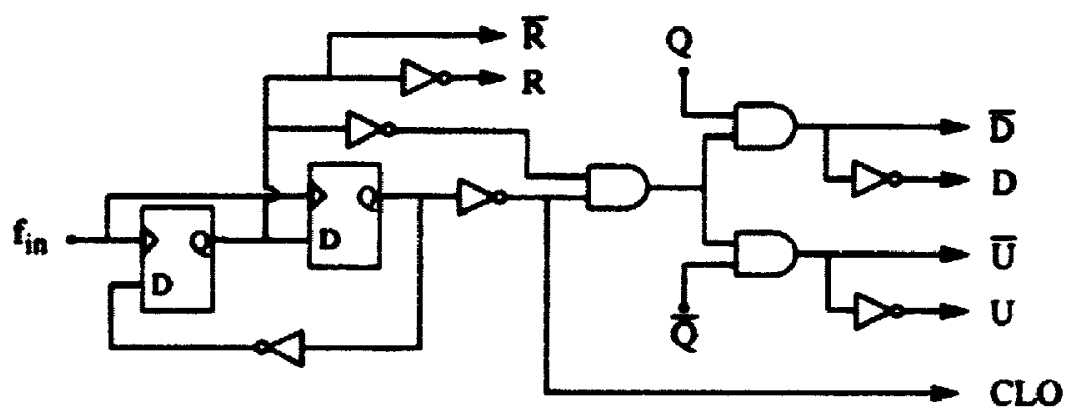

Figure 4.11 : The reference and feedback signal ECL PAL circuit.

for the derivation of a $10 \mathrm{MHZ}$ signal with the $25 \%$ duty cycle. This pulse was used to generate charge pump feedback signals. Figure 4.11 shows the diagram for the ECL PAL interface circuit.

\section{3 $\Delta \Sigma$ Frequency Discriminator Circuit Realization}

What is presented in this section is a set of measurements and analysis qualifying the performance of the $\Delta \boldsymbol{\Sigma}$ frequency discriminator circuit shown in Appendix $C$. The circuit was realized as a two layer printed circuit board using small outline surface mount components. 
Figure 4.12 shows the timing diagram for circuit operation under an FM input of $100 \mathrm{KHz}$ deviation and $70 \mathrm{KHz}$ modulation rate on a $107 \mathrm{MHZ}$ carrier. From the measurement or Figure 4.12, five continuous points of operation have been identified for a single reference cycle. Point 1 indicates the rising edge of the reference signal $\bar{R}$, which is used as a clock to latch the comparator/quantizer element. The change in the output of the quantizer, $Q$, seen at point 2 securs on the rising edge of $\bar{R}$.

Point 3 indicates the rising edge of $\bar{V}$, the output of the DMD. The difference between the rising edges of $\bar{R}$ and $\bar{V}$ contain a DC phase offset component and a modulation component $\tau_{k}$ which result in the pulse width modulation of the PFD outputs $U_{l}$, and $D_{l}$.

Point 4 indicates the position for the rising edge of the $25 \%$ duty cycle feedback signals $U_{2}$ and $D_{2}$. It is critical that the falling edge of these signals occur before the next rising edge of $\bar{R}$ seen at point 5 . This measurement indicates that a full cycle of the second onder structure occurs within one period of the reference frequency, and therefore occur with a single delay around the loop. This criterion is critical for stable operation ot a feedback $\Delta \Sigma$ structure. Measurements also indicated that the useful tuning range for the DC phase offset was $50 \%$ of the reference period. Noise shaping operation did not occur beyond this range. In the timing diagram, at point 3, the edge information $\tau_{k}$ on $\bar{V}$ contains the coarse quantization noise as well as the digital FM modulation signal.

Figure 4.13 presents a 120 set ensemble average of 16000 point FFTs on the output bitstream of the discriminator. A total of $2^{20}$ bits were captured and stored to allow a post processing average of 120 separate 16000 point FFTs. The measurement clearly exhibits quantization noise shaping. The 70KH: modulation tone (effectively a $140 \mathrm{~kb} / \mathrm{s}$ rate) is also present as expected. The signal power level of the tone appears to be coout $28 \mathrm{~dB}$ above the noise level at $70 \mathrm{KHz}$, yet this result may appear misleading due to the equivalent noise bandwidth (ENBW) of each frequency bin. A more meaningful way to evaluate the measurement is to evaluate the effective bit resolution of the output signal after decimation. This type of evaluation san be simplified if it is assumed that the output is low pass filtered by a brick wall filter with effective bandwidth $B W_{w n}$, that cuts off at the modulation rate of $140 \mathrm{~Kb} / \mathrm{s}$ (i.e. $140 \mathrm{KHz}$ ) with the passband noise being white.

For the discriminator the full scale carrier devistion is $\pm 5 \mathrm{MHz}$ (10MHz). Also, the full scale modulation deviation is $\pm 50 \mathrm{KHz}(100 \mathrm{KHz})$. The ratio of these full scale 


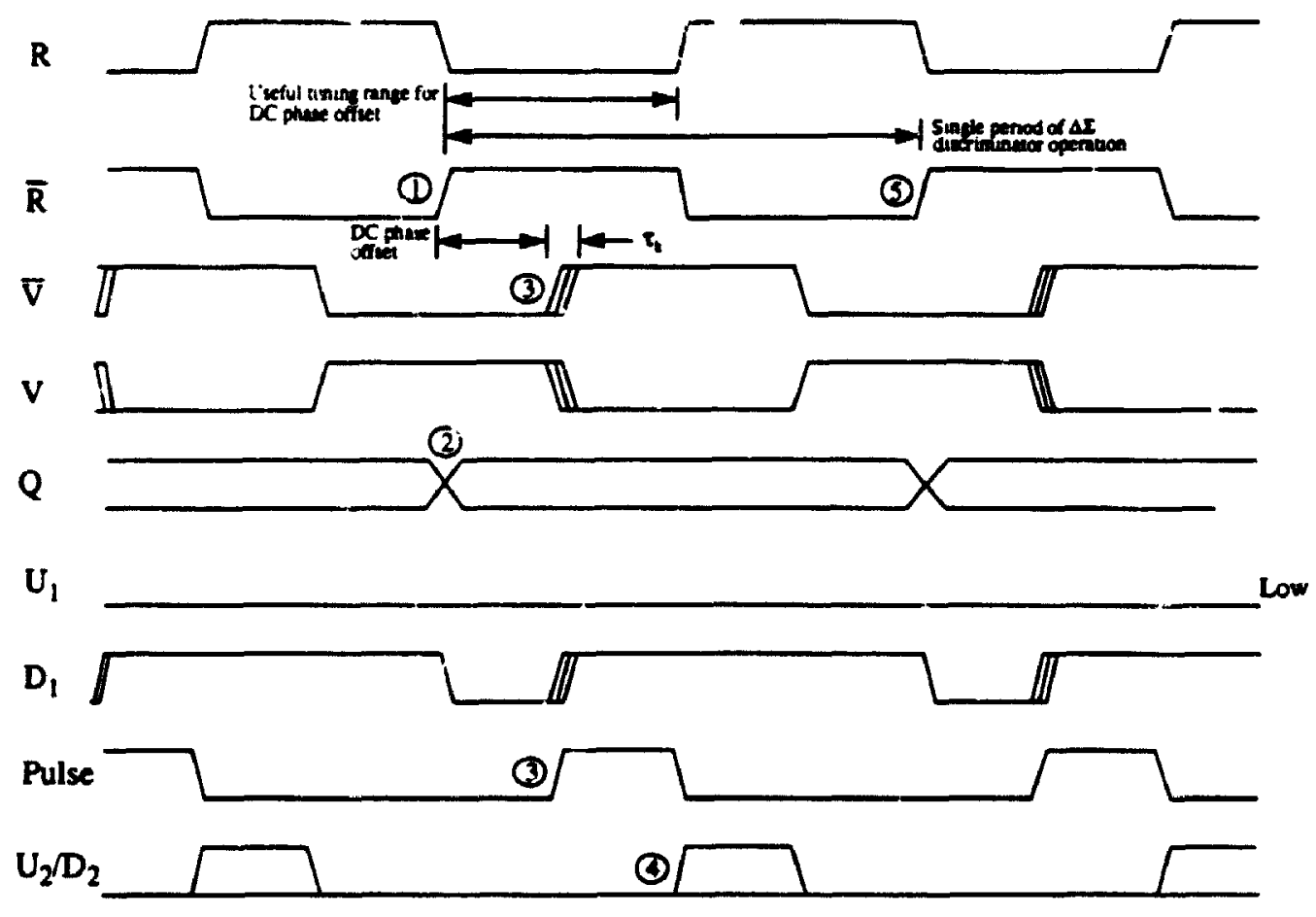

Figure 4.12 : Measured timing diagram for the $\Delta \Sigma$ frequency discriminator.

deviations is related to the magnitude of the signal power level by

$$
S_{d E}=-20 \log \left(\frac{D_{d i s, f s}}{D_{m o d, f s}}\right)=-40 d B
$$

This relationship assumes that the relative oower level of a full scale deviation is zero dB. From Figure 4.13 the relative signal power level $S_{d B}$ in the $70 \mathrm{KH}_{2}$ frequency bin is approximately $-24 d B$. This measured result indicates a $12 d B$ scaling difference with respect to the calculated value of equation (4.28). This results from the assumption that a full scale deviation results in a relative power level of zero dB. From the same curve of Figure 4.13, assuming a white noise passband $B W_{w n}$ of $140 \mathrm{kHz}$, the approximate noise power level $\eta_{d B}$ is $-52 \mathrm{dBc}$. In order to calculate the SNR for the modulation frequency bin the ENBW must be determined. 


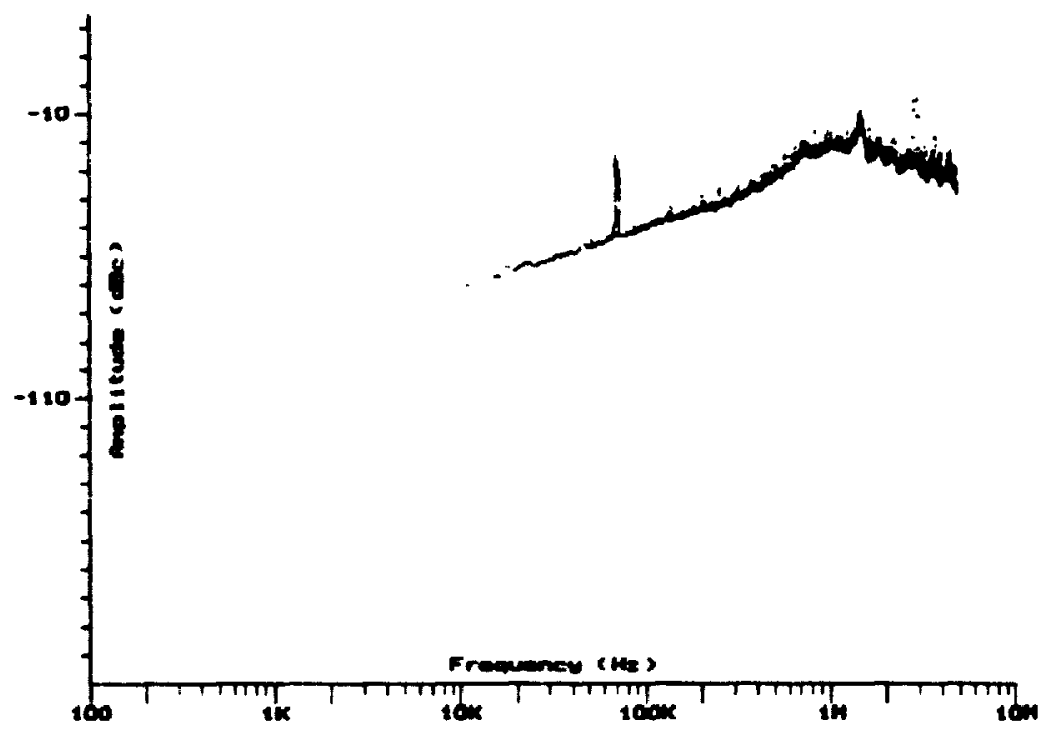

Figure 4.13: The power spectral density of the captured C.scriminator output bitstream.

The ENBW per bin can be determined using [Har78]

$$
E N B W=\frac{\sum_{n} w^{2}(n T)}{\left[\sum_{n} w(n T)\right]^{2}} \times \text { binwidth }
$$

where $w(t)$, the temporal weightings [Nut91], are given by

$$
w(n t)=\frac{1}{L} \sum_{n=0}^{n} a_{n} \cos \left(\frac{2 \pi n t}{L}\right) ;(|n| \leq L / 2)
$$

and $L$ is the duration of the temporal weightings. Harris [Har78] conveniently computes the coefficient of (4.29), normalized to the bin width, for a minimum 4 sample Blackman-Harris window. to be 2.0. The ENBW now becomes 


$$
E N B W=2 \times \frac{f_{s}}{N}
$$

where frequency-bin width $f_{s} / N$ is a function of $N$, the number of points in the FFT, and $f_{s}$ is the sampling frequency. From (4.31) ENBW is determined to be $1250 \mathrm{~Hz}$. From the :adsun:d values and assuming a white noise bandwidth $B W_{w n}$ up to and including the modulation frequency, the signal to noise ratio becomes

$$
\begin{gathered}
S N R_{d B}=\frac{10 \log \left[\left(v_{s}^{2}\right) \times k\right]}{10 \log \left[\left(\frac{v_{n}^{2}}{E N B W} \times B W_{w n}\right) \times k\right]} \\
S N R_{d B}=\frac{S_{d B}+10 \log (k)}{\eta_{d B}-10 \log (E N B W)+10 \log \left(B W_{w n}\right)+10 \log (k)}=7.51 d B
\end{gathered}
$$

where $k$ is a magnitude scaling factor introduced by the software used to evaluate the FFT. This term cancels out in equation (4.33). In order to determine the effective number of bits from 7.51 dE, from Proakis [Pro89] for a pulse code modulated signal, the rms error can be determined using

$$
\text { error }_{r m s, d B}=20 \log \left(\frac{\Delta}{\sqrt{12}}\right)=20 \log \left(\frac{2^{-N}}{\sqrt{12}}\right)
$$

Here $\Delta$ is the quantizer step size. Separating and solving for the right-hand side of (4.33) we have

$$
\text { error }_{r m s, d B}=-10.8-6 N_{B}
$$

Where $N_{B}$ is the number of bits used to represent each sample. Equating the right hand side of (4.34) to the noise le.'el (relative to the s' znal magnitude) of (4.32) leads to an effective number of bits/sample $N_{B}$ of approximately 0.55 . This poor result indicaws several problems with the circuit. The magnitude of the 
signal power level is directly related to the magnitude of the frequency deviation of the modulation signal. Increasing the deviation would lead to greater SNR. This particular discriminator design would also provide better quality discrimination for lower data rates. This problem can be seen in the result of Figure 4.13 where the existence of only a first order quantization noise shaping at the modulation frequency results in high levels of noise and therefore reduced SNR. In the range $500 \mathrm{KHz}$ to $1.5 \mathrm{MHz}$ the quantization noise has second order shaping, yet below this point frequency discriminator operation degrades to first order operation. This quantization noise degradation dramatically reduces the effective value of $N_{B}$ over the baseband range. The degradation may be a result of leakage on the integrating capacitors of the charge pump circuit. Under ideal operation each packet of current from $I_{I}$ and $I_{2}$ causes a voltage step on the integrating capacitors for each cycle of the reference frequency. If these capacitors are loaded by a finite resistance during the reference cycle when the integrating capacitors are neither charging or discharging (i.e. $U, D$ high and $\bar{U}, \bar{D}$ low) then the current can leak off the capacitors with a time constant $\tau_{L}$ given by

$$
\tau_{L}=R_{L O A D} \times C
$$

This time constant is inversely proportional to the frequency where the chargepump breaks down and ceases to act as an integrator.

These results indicate that this particular implementation of the frequency discriminator is well suited to large deviation, low data rate digital frequency modulation. Considerably better performance is expected from VLSI implementation work being done by Beards [BC92]. 


\section{Chapter 5}

\section{Experimental Investigation for Validation of Broadband FM Conversion}

In chapter 2, an experimental testbed was presented (Figure 2.6) for use in testing the concept of single conversion FM broadband mixing. For the fractional-N PLL synthesizers used in the testbed, in static operation, one expects synthesizer frequency outputs with zero frequency and phase error, resulting in a single fixed IF tone after the frequency conversion process. For non-steady-state frequency operation it can be expected that some level of frequency and residual phase error will occur which is a function of the open loop gain of the synthesiz-r. From linear theory, frequency independent finite open loop gain limitations result in a fixed percentage frequency error of the magnitude frequency deviation of the modulating signal. Frequency dependent finite open loop gain limitations (ie; a function of the PLL phase error transfer function) result in a frequency error which is dependant upon the modulation rate of the modulating signal. In the case of the testbed the magnitude deviation and repetition rate are varied with respect to a wideband sinusoidal FM test vector. Minor frt ${ }_{2}$ dency differences between the $T_{x}$ and $R_{x}$ synthesizers, a function of their respective open loop gains, would directly result in IF distortion.

In this chapter, because ideal synchronization has been assumed, the mechanisms which potentially impair broadband operation and lead to IF distortion are isolated and evaluated with the testbed, using a single Fourier component, a broadband sinusoidal FM test vector. After experimental variables and measurements are defined with respect to the experimental testbed, test results are presented as they relate to key issues pertinent to the operation of the $\Delta \mathbf{\Sigma}$ frequency synthesizer and $\Delta \Sigma$ frequency discriminator, as part of a broadband digital FM transceiver. 


\subsection{Experimental Variables Defined}

Using the test set-up of Figure 5.1 (reprinted here from Figure 2.6) the $\boldsymbol{R}_{x}$ synthesizer was mixed to an IF frequency of $105 \mathrm{MHz}$. At the $T_{x}$ end, an offset frequency of $105 \mathrm{MHz}$ was added to the $T_{x}$ synthesizer which allowed easy evaluation of broadband conversion at an IF center frequency that suited the input frequency range of the $\Delta \Sigma$ frequency discriminator, as indicated by Figure 4.10. At the same time this allowed nominally identical synthesizers to be used at both the $T_{x}$ and $R_{x}$ ends. A broadband sinusoidal FM test vector (see section 2.2.3), with no data present, was used as a vehicle to drive both synthesizers, thereby providing a well defined environment for determining the distortion and SNR potential $c^{c}$ the broadband conversion architecture. Issues and details regarding the mixers are discussed in Section 5.6.

For the purposes of testing broadband conversion both the $T_{x}$ and $R_{x}$ synthesizers of Figure 5.1 were fine synchronized by applying the same $10 \mathrm{MHz}$ reference irequency to both components. The dependant variable measured using the testbed was the IF output signal, designed to be at $105 \mathrm{MHz}$. The level of time dependent frequency movement with respect to the desired IF center tone, a result of the test vector repetition rate and frequency deviation, was specifically used as a meacyise of impairment (ie; the analog mismatch between the $T_{x}$ and $R_{x}$ synthesizers for

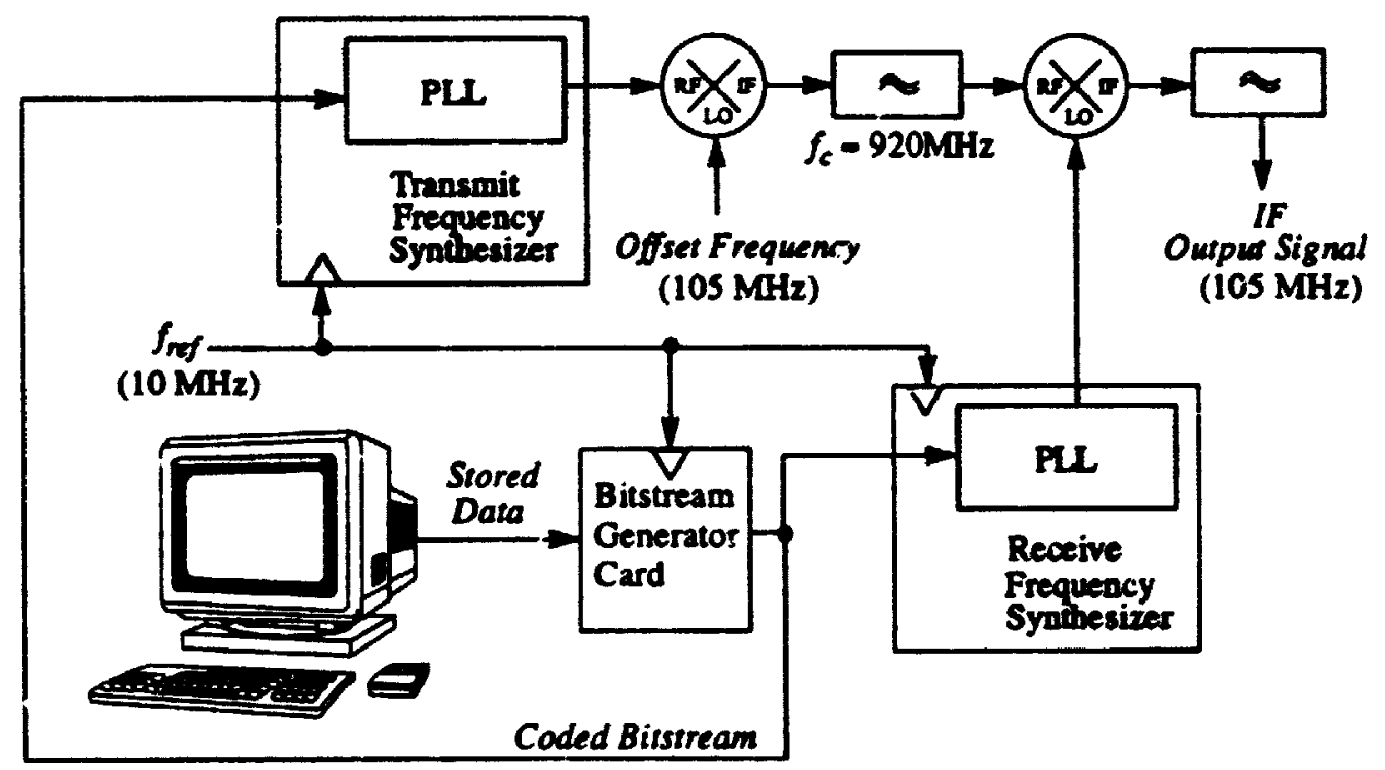

Figure 5.1 : Block diagram of the experimental testbed for broadband coded FM. 
various values of the independant variables of the test vector). The independant variables usid as controls in the experimental measurements were the repetition rate (no data present), and the peak deviation of the sinusoidal wideband FM test vector, as controlled by the content of the double precision digitally encoded $\Delta \Sigma$ modulated bitstream. In particular, frequency deviations of $\pm 2.5 \mathrm{MHz}, \pm 625 \mathrm{KHz}$. $\pm 156 \mathrm{KHz}$, and repetition rates of $1 \mathrm{KHz}$ and $10 \mathrm{KHz}$ were used. Note that in the absence of data being added to the $\Delta \Sigma$ modulator input, since the same sinusoidal test vector is used in the $T_{x}$ and $R_{x}$ modulators, the resulting IF signal would be a pure $105 \mathrm{MHz}$ sine wave in the ideal case. The characteristics of the sinusoidal FM test vector are shown in Figure 2.1 and described in section 2.2.3.

\subsection{Distortion Analysis for Broadband FM Conversion}

Figure 5.2 shows two modified block diagram models of the PLL in terms of its phase error output and DMD phase components. The open loop gain $H_{O L}$ from Figure 5.2 (b) is given by [RC92c]

$$
H_{O L}=\frac{k_{\theta} F(s) k_{\nu}}{s}
$$

The full definition of $H_{O L}$ from equation (3.14), for the PLL synthesizers usea, is given by

$$
H_{O L}(j \omega)=-\frac{\omega_{n}^{2}{ }^{k} v_{p}{ }^{2}}{(n+\beta) \tau_{1} \omega^{2}}\left[\frac{1+j \omega \tau_{2}}{j \omega\left[2 \zeta \omega_{n}+\tau_{3}\left(\omega_{n}^{2}-\omega^{2}\right)\right]+\omega_{n}^{2}-\omega^{2}-2 \zeta \tau_{3} \omega_{n} \omega^{2}}\right]
$$

The manum phase error $\theta_{e, m}$ as a function of the maximum phase variation $\theta_{b, m}$ resulting from the broadband sinusoidal FM signal modulating the $\Delta \mathbf{\Sigma}$ modulator bitstream $b(t)$ is given by

$$
\theta_{e, m}=\frac{1}{1+H_{O L}} \theta_{b, m}=\frac{1}{1+H_{O L}} \beta_{F M}
$$

where $\beta_{F M}$ was defined in section 2.1. 


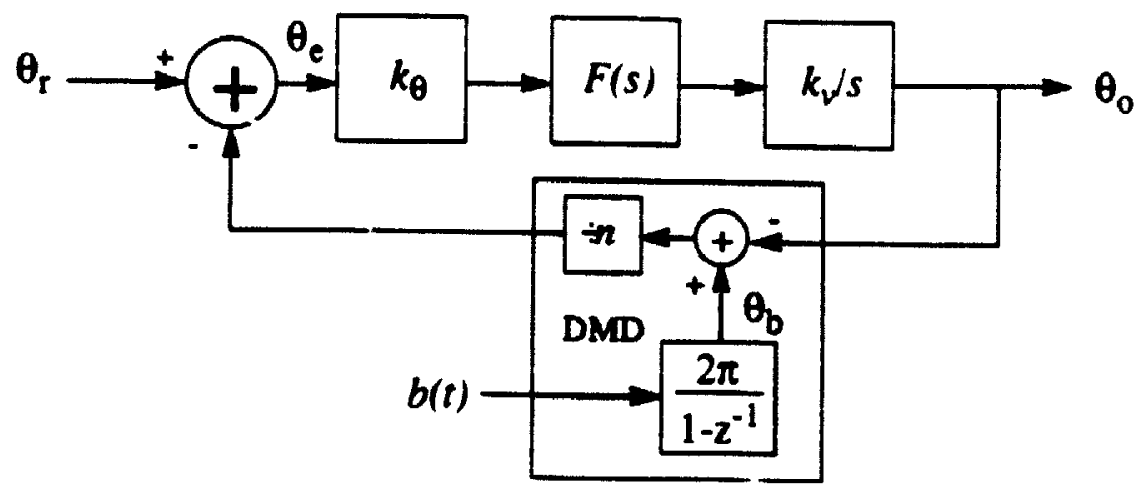

(a)

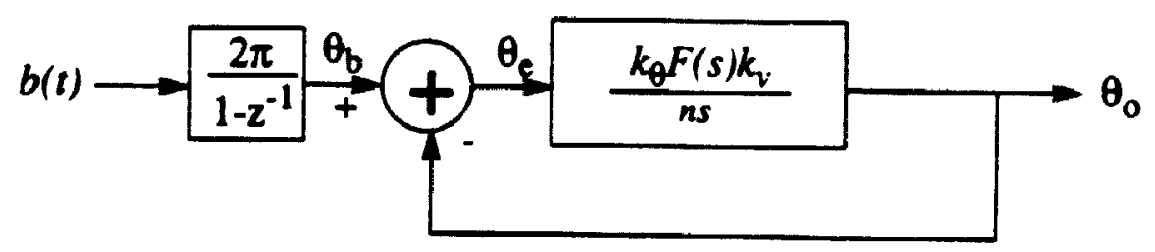

(b)

Figure 5.2 : Block diagram of the model of a Fractional-N PLL in terms of its phase components.

This phase error in addition to the error due to path delay will be discussed later (see section 5.4).

The phase variation introduced by the sinusoidal FM test vector is

$$
\theta_{b}(t)=\int_{0}^{t} \omega(t) d t=\int_{0}^{t} 2 \pi \Delta f \sin \left(2 \pi f_{m} t\right) d t+2 \pi f_{c} t
$$

Performing the integration and dropping the DC gives

$$
\theta_{b}(t)=\frac{\Delta f}{f_{m}} \sin \left(2 \pi f_{m} t\right)
$$




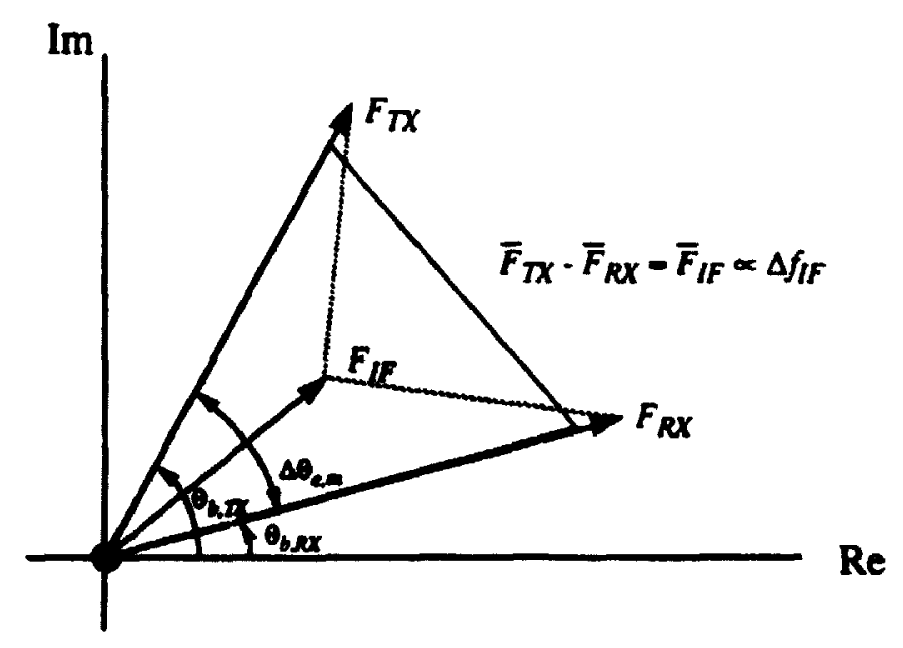

Figure 5.3 : Phasor representation for analysis of IF broadband conversion distortion.

If the maximum phase deviation due to the sinusoidal FM test vector is considered, then

$$
\theta_{b, m}=\frac{\Delta f}{f_{m}}=\boldsymbol{B}_{F M}
$$

which is the classical definition of the maximum phase deviation for sinusoidal FM. Equation (5.3) shows that the phase error $\theta_{e}$ only approaches zero as the open loop gain $H_{O L}$ approaches infinity. For all practical purposes the circuit gain is finite, and the high pass nature of the phase error transfer function gives decreasing phase error suppression for increasing frequencies. Using this analysis it can be seen that, for finite $\mathfrak{E}$ ain, residual levels of phase error would directly translate into minor frequency errors in the closed loop output of a $T_{x}$ synthesizer, with resfect to an alternate yet similarly constructed $\boldsymbol{R}_{x}$ synthesizer. Assuming synchronized, non-steady state operation, then the phase difference (path delay independant) between the $T_{x}$ and $R_{x}$ synthesizers is given by

$$
\Delta \theta_{e, m}=\left[\frac{1}{1+H_{O L, R x}}-\frac{1}{1+H_{O L, T x}}\right] \theta_{b, m}
$$


If one views the broadband sinusoidal FM driven $T_{x}$ and $R_{x}$ synthesizer outputs as rotating phasors in frequency, as shown in Figure 5.3, then the minor phase differences $\Delta \theta_{e, m}$ existing between synthesizers results in a phase dependant IF frequency $F_{I F}$, with magnitude bandwidth $\Delta f_{I F}$. Ideally if $F_{R X}$ and $F_{T X}$ have the same phase (ie; $\Delta \theta_{e, m}-0$ ) then the vector difference resulting from the broadband conversion leads to zero IF distortion (a pure tone as symbolized by a point at the origin of the axes). For finite phase differences the magnitude of $F_{I F}$ is directly related to the phase error $\Delta \theta_{e, m}$ and results in a rotating vector at IF with a given distortion bandwidth. Any desired level of SNR at the IF stage must adjust the frequency deviation of a data bit in order to compensate for the magnitude of this frequency distortion.

Alternatively, the frequency imbalance inherent in this type of PLL synthesizer can also be understood by defining the magnitude and phase of the frequency difference that can occur between a desired and a realized output. If $f_{T X, d}(t)$ is defined as the desired output frequency and $f_{T X, a}(t)$ the actual or realized output frequency, then the difference between these components $f_{T X, e}(t)$ can be defined as the frequency error at the synthesizer output due to the filter action, and is given by

$$
f_{T X, e}(t)=f_{T X, d}(t)-f_{T X, a}(t)
$$

and the peak frequency deviation given by

$$
\Delta f_{T X, e} \because: f_{T X, d}(t)\left|\frac{1}{1+H_{O L, T X} \angle \theta_{O L, T X}}\right|
$$

These relationships are shown in Figures 5.4 a) and b) and the phase error $\theta_{T X, e}$ between the desired and actual signals defined by

$$
\theta_{T X, e}=\angle \frac{1}{1+H_{O L, T X} \angle \theta_{O L, T X}}
$$

Therefore the closed loop transfer function becomes the soirce of the phase and magnitude shift between $f_{T X, d}(t)$ and $f_{T X, a}(t)$ (note that the phase shift causec by additional path delay is an issue discussed separately). If this analysis is carried further, then the difference between two dependar.t (ie; same reference frequency) 


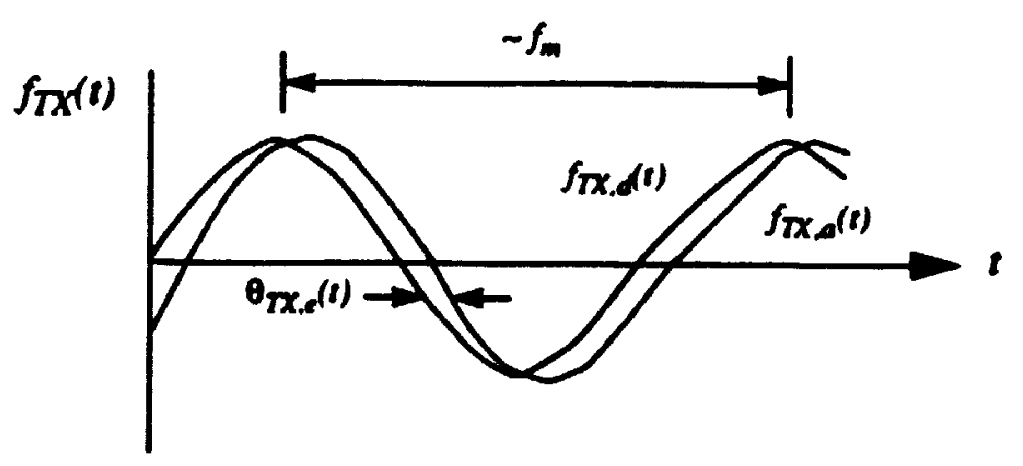

(a)

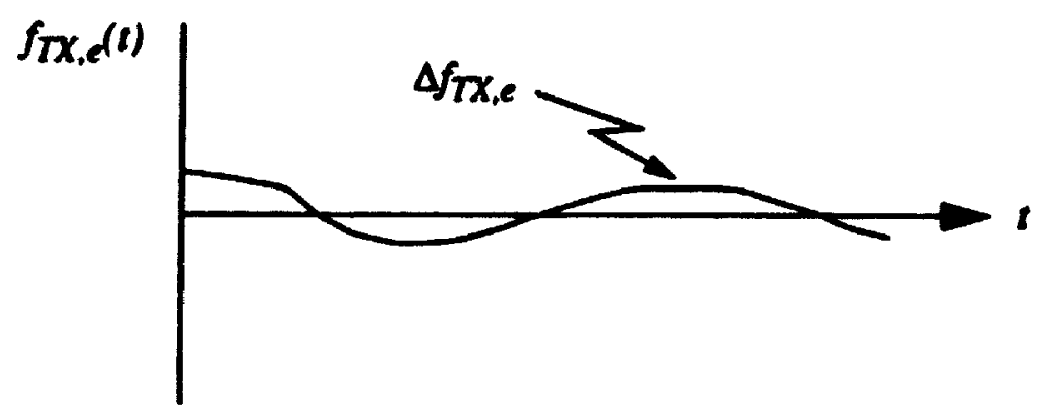

(b)

Figure $5.4:$ a) The phase relationship between the desired and actual transmit frequency, b) the magnitude frequency error between the desired and actual transmit frequency.

transfer functions in both phase and magnitude, can be viewed as the difference between the $T_{x}$ and $R_{x}$ 'actual' frequencies. This difference is in effect the overall frequency deviation error at the particular modulating frequency. $\beta_{n}$ or residual $\beta$, is the difference in the peak frequency deviation caused by the filtering action of the $T_{x}$ and $R_{x}$ PLL sections, and can be defined using the peak frequency errors of both the $T_{x}$ and $R_{x}$ synthesizers.

$$
\beta_{r}=\left|\frac{\Delta f_{T X, e}<\theta_{T X, e}-\Delta f_{R X, e}<\theta_{R X, e}}{f_{m}}\right|
$$

Alternatively, using the definition of peak frequency error provided in equation 5.9. c quation 5.11 can be expanded to 


$$
\beta_{r}=\left|\frac{f_{T X, d}(t)\left|\frac{1}{1+H_{O L, T X}}\right|<\theta_{T X, e}-f_{R X, d}(t)\left|\frac{1}{1+H_{O L, R X}}\right|<\theta_{R X, e}}{f_{m}}\right|
$$

For a broadband FM conversion process the desired frequency deviations $f_{T X . d}(t)$ and $f_{R X, d}(t)$ at the $T_{x}$ and $R_{x}$ synthesizers respectively will be identical, and therefore equation 5.12 can be simplified to

$$
\beta_{r}=\frac{f_{R X, d}(t)}{f_{m}}\left|\frac{1 \angle \theta_{T X, e}}{1+H_{O L, T X}}-\frac{1 \angle \theta_{R X, e}}{1+H_{O L, R X}}\right|
$$

Equation 5.13 provides a closed form solution for evaluating the peak phase deviation at the IF stage as a function of each PLL open loop gain. FM deviation. and FM rate. The peak phase deviation at the IF output $\beta_{I F}$ can be evaluated from equation 5.13 by substituting the peak frequency deviation of the FM test vector for $f_{R X, d}(t)$, and providing correct values for the open loop gain, the phase error, and the FM rate.

The non-steady state operating point at which the above phase difference becomes dominant in broadband mixing is a function of the individual open loop gains. which are directly related to the frequency deviation $\Delta f$ and the repetition rate $f m$ of the test vector. This is the subject under investigation in section 5.4. From a testing perspective it bea omes important to verify the effects of these minor differenct; on the If signal during broadband mixing, and at what values of repetition rate $f m$ and frequency deviation $\Delta f$ these effects might reduce the IF SNR to levels that are unpractical for a digital FM radio receiver. It would be expected that the worst effects would show up at higher levels of $f_{m}$ and $\Delta f$.

The phasor diagram of Figure $\mathbf{5 . 3}$ assumes the magnitude of the voltage signals that develop the $T_{x}$ and $R_{x}$ frequencies are constant amplitude over the FM bandwidth. Variations in the magnitude synthesizer output voltages over this bandwidth will lead to AM modulation of the resulting IF waveform. The implications of this form of distortion (AM to PM conversion) through subsequent non-linear action will not be addressed in this thesis. 


\subsection{Frequency Synthesizer Characterization using a Sinusoidal Broadband FM Test Vector}

Figure 5.5 shows the power spectrum of the individual $T_{x}$ and $R_{x}$ synthesizers in steady-state frequency operation at $815 \mathrm{MHz}$. The spectrum of each synthesizer indicates loop bandwidths of about $90 \mathrm{KHz}$ and phase noise power levels less optimum than the single sideband phase noise results of Figure 3.17 (due to empirical tuning of loop gain for wideband operation). The contour of the phase noise shows the interaction of the $\Delta \Sigma$ modulator high pass shaped quantization noise, the VCO phase noise, and the closed loop suppressing action of the PLL. For fixed frequency operation the synthesizers provided high quality single tone outputs, similar to Figure 5.5, over most of the operating bandwidth. Using the testbed of Figure 5.1 each synthesizer was cha xcterized for sinusoidal frequency deviations of $\pm 2.5 \mathrm{MHz}, \pm 625 \mathrm{KHz}$, and $\pm 156 \mathrm{KHz}$ for both $1 \mathrm{KHz}$ and $10 \mathrm{KHz}$. Figures 5.6, 5.7 and Figures D.1 through D.4 (Appendix D) show the frequency versus time plots of the individual synthesizers in wideband operation. These plots are sinusoidal as expected, aside from the obvious sampling error for the \pm 625 $\mathrm{KHz}$ and $\pm 156 \mathrm{KHz}$ at $10 \mathrm{KHz}$ rate signals, shown in Figures D.1 and D.3. Because a synchronizing signal was not used for the individual frequency versus time measurements the apparent phase offset between $T_{x}$ and $R_{x}$ outputs has no significant meaning. The power spectrums of Figures 5.8 and Figures D.5 through D.9 all show responses which indicate well matched $T_{x}$ and $R_{x}$ synthesizers. These results were generated independently and compared in non-real time and therefore it -se characterizations can only provide an indication of correct operation of the test setup compenents for the various test vectors. They cannot provide sufficient evidence of the matching of the complex combination of time constants and gain factors within each of the $T_{x}$ and $R_{x}$ phase locked loops. The necessary evidence of synthesizer match/mismatch conditions must come from a controlled examination of the mixing process involving each synthesizer, first mixed with itself and secondly mixed with each other, for the same set of test vectors. Results from such an examination will provide initial insights into a more specific and pragmatic approach for continued research into broadband conversion. 


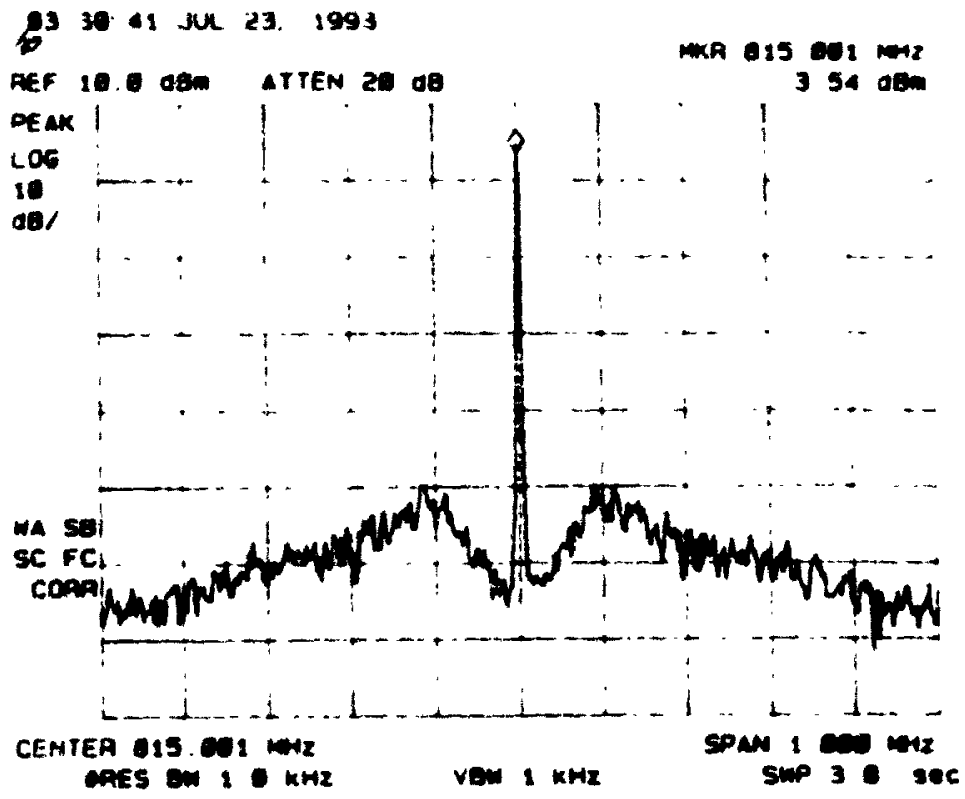

(a)

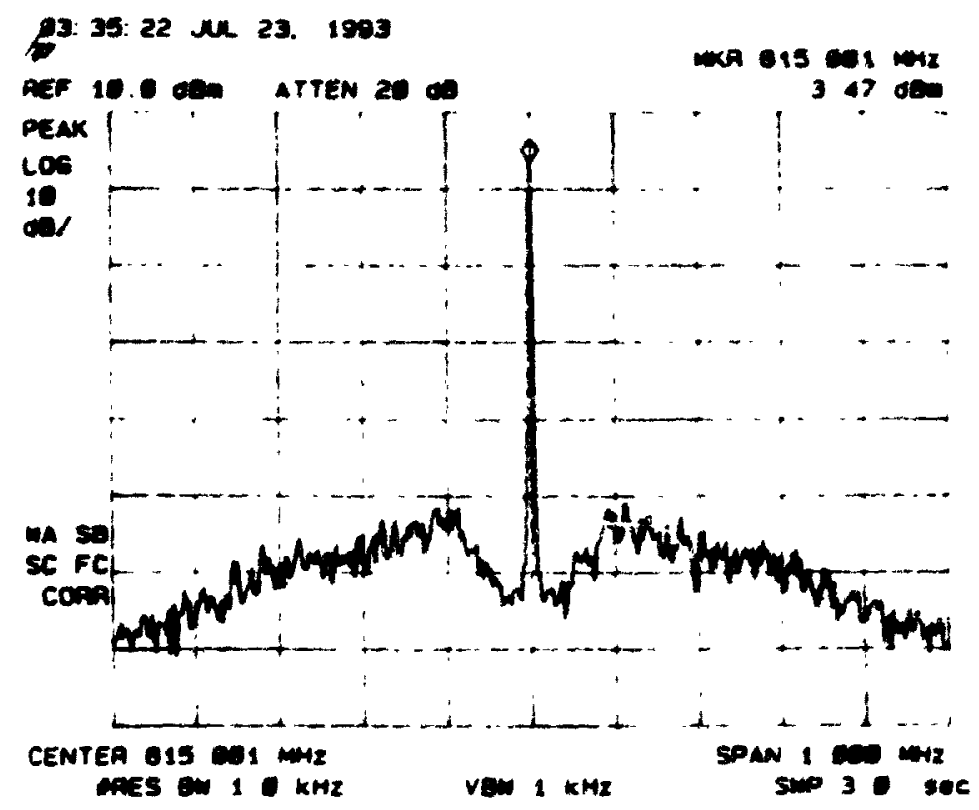

(b)

Figure 5.5 : Plots of the power spectrums of the individual a) $R_{x}$ and b) $T_{x}$ frequency synthesizers operating in fixed frequency mode. 

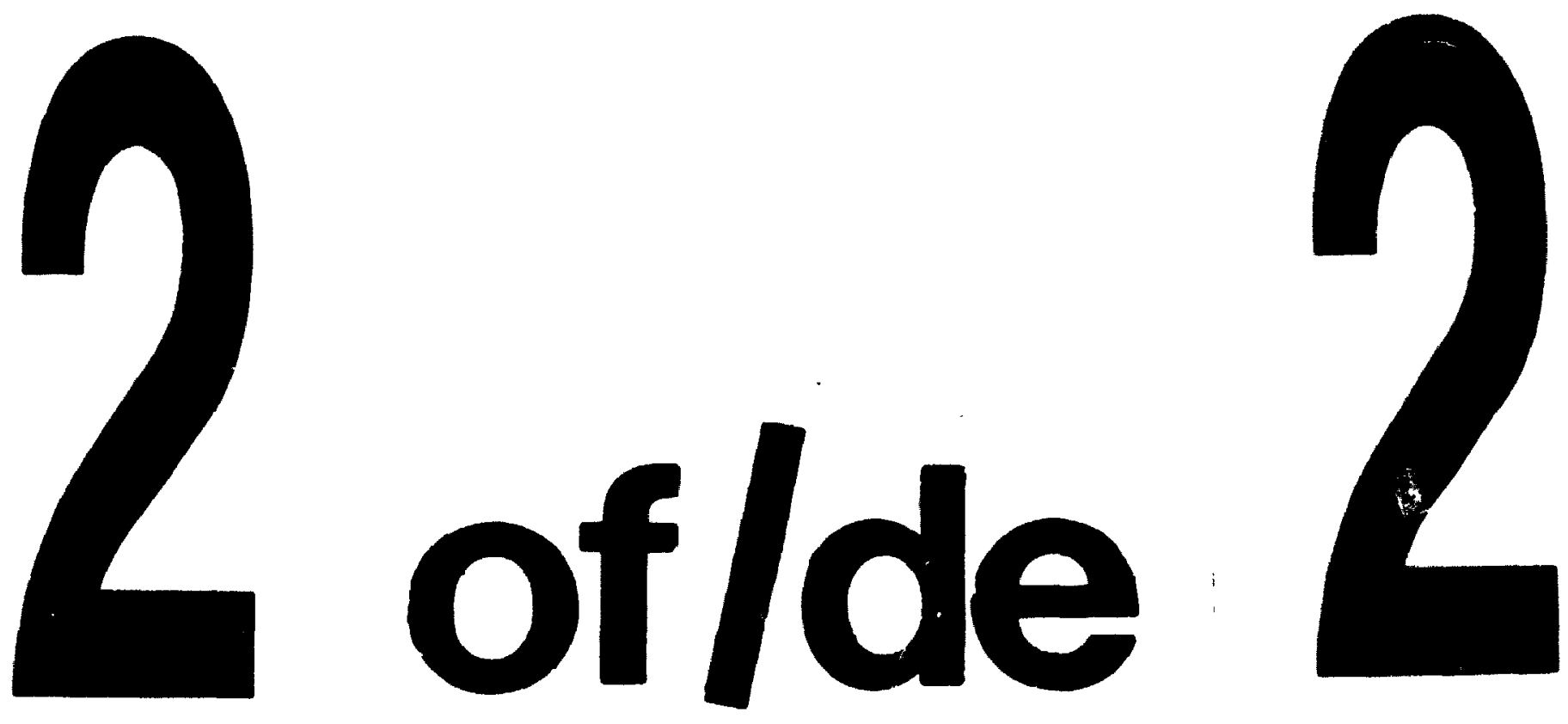

PM-1 3Y" "x4" PHOTOCRAPHIC MiCROCOPY TARRET

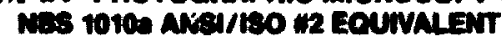

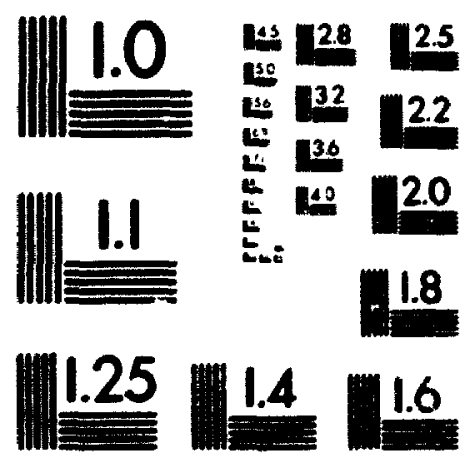

PRECISION"W RESOUUTION TARCETS 


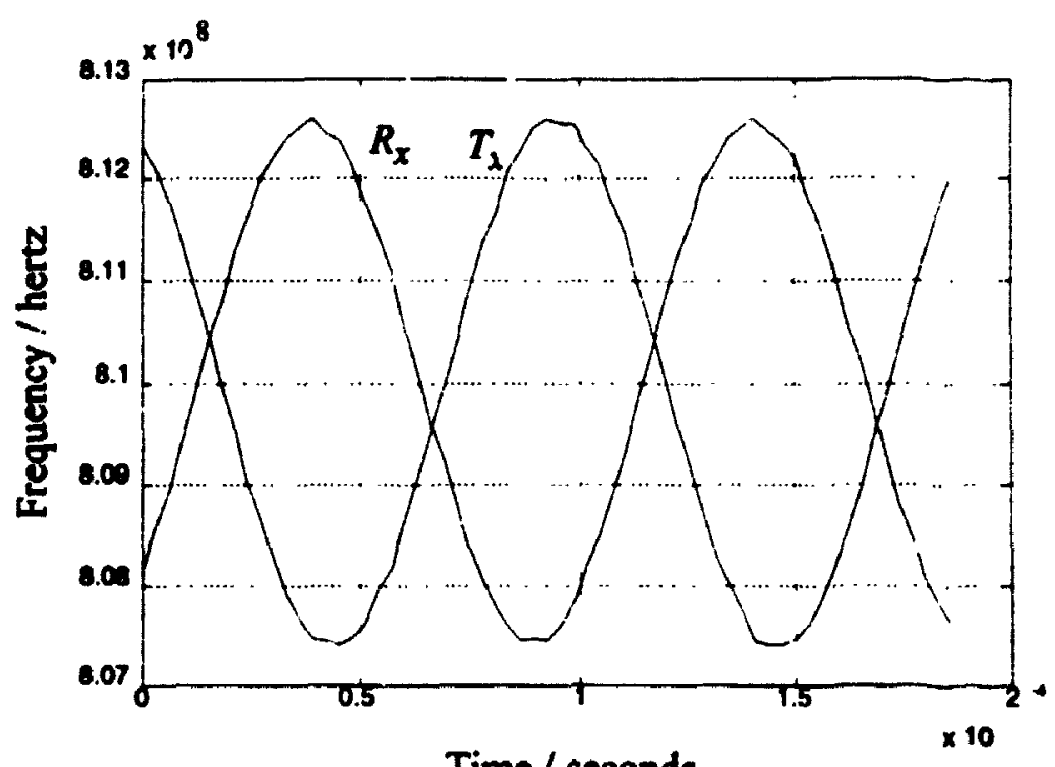

Figure 5.6: Plots of frequency versus time for the $R_{x}$ and $T_{x}$ frequency synthesizers using a $10 \mathrm{KHz}$ repetition rate and peak deviation of $\pm 2.5 \mathrm{MHz}$.

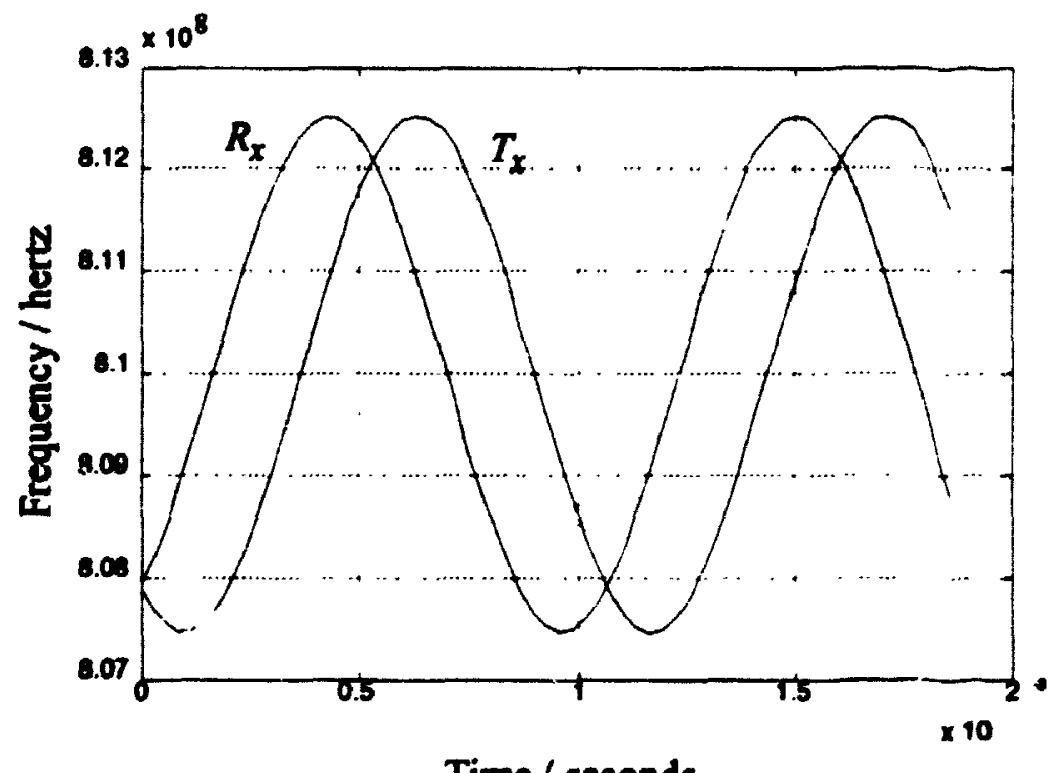

Figure 5.7 : Plots of frequency versus time for the $R_{x}$ and $T_{x}$ frequency synthesizers using a $1 \mathrm{KHz}$ repetition rate and peak deviation of $\pm 2.5 \mathrm{MHz}$. 


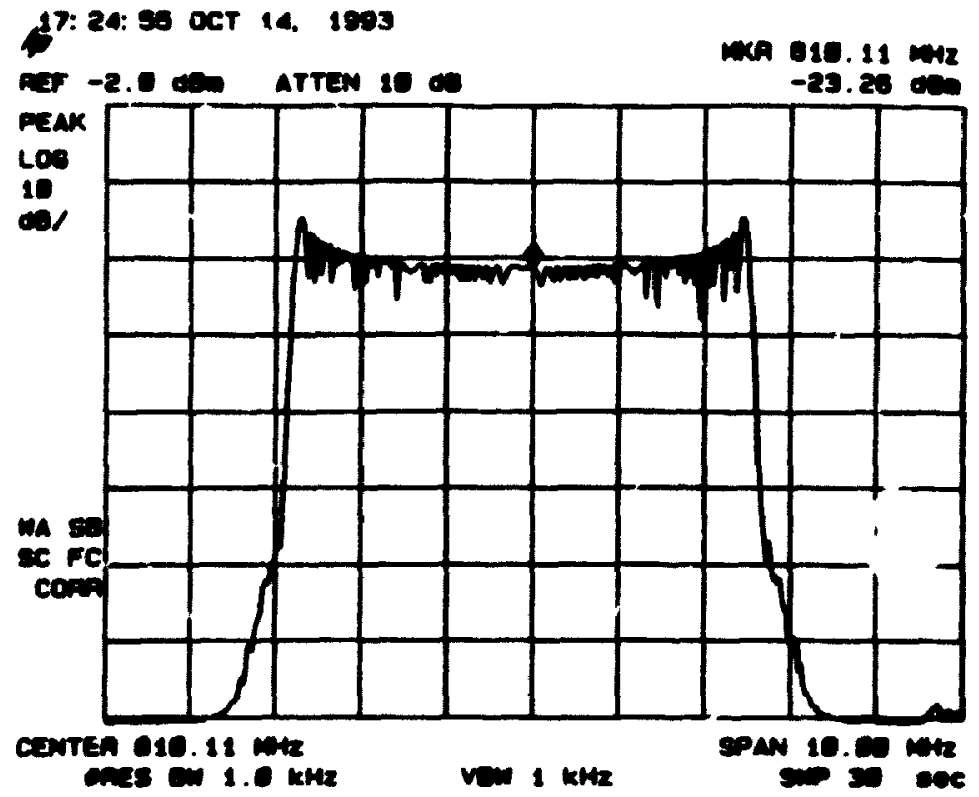

(a)

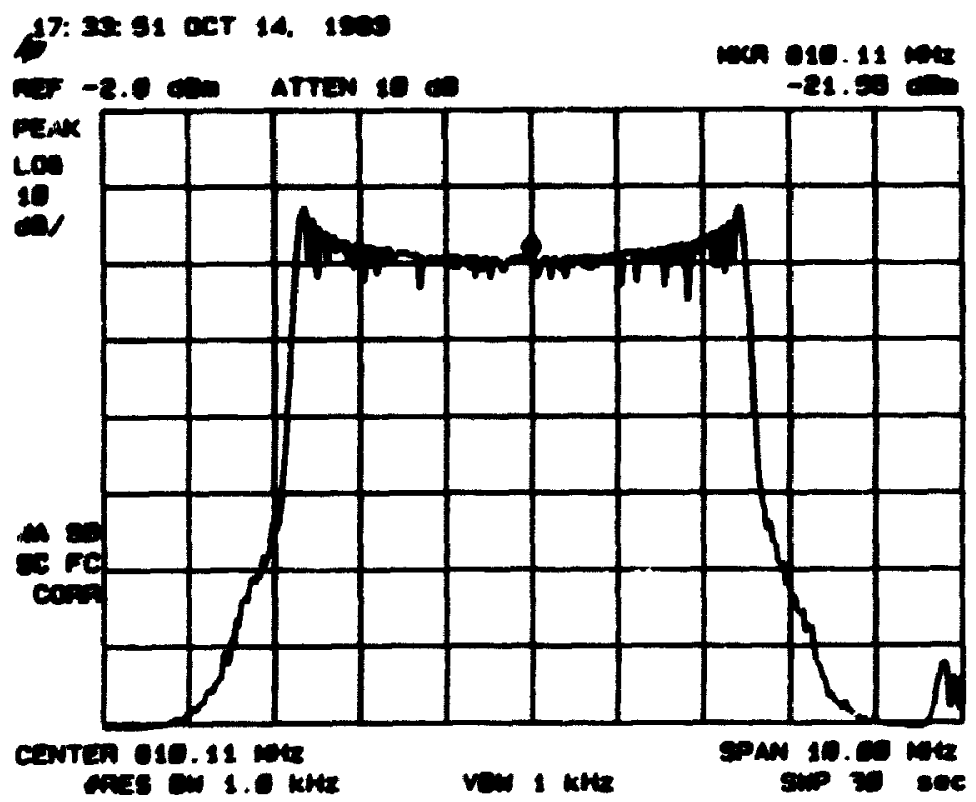

(b)

Figure 5.8 : Plots of the power spectrum for a) the $R_{x}$ and $b$ ) the $T_{x}$ frequency gynthesizers operating with a peak deviation of $\pm 2.5 \mathrm{MHz}$ and $10 \mathrm{KHz}$ repetition rate. 


\subsection{Ideal Broadband Conversion Measurement}

As discussed in section 2.2.4, for testing purposes ideal synchronization has been assumed between the $T_{x}$ and $R_{x}$ sections of the test set-up using a common reference frequency. In this section broadband conversion is investigated under ideal PLL matching conditions by mixing the effective $T_{x} / R_{x}$ synthesizer with itself (self mixing). From equation 5.7 the only differences between the synthesizer signals at the mixer $T_{x}$ and $R_{x}$ inputs will be the distortion added by the offset frequency mixer, the $920 \mathrm{MHz}$ filter (FBPF-915/30) [KCOM], and the interconnecting semi-rigid coaxial cables. The 3 inch coaxial lines used in the test setup exhibit phase delays of $-0.5 \mathrm{~ns}$, while the $920 \mathrm{MHz}$ filter has $-55 \mathrm{~ns}$ of delay at the center frequency. Test setup sensitivity to these path impairments will show up as a measurement artifact but should be non-domirant with respect to the broadband measurement results. Figure 5.9 shows how ideal broadband conversion can be measured using the test set-up of Figure 5.1. Evaluation of effectively ideally matched synthesizers, although simple in concept, does provide a baseline for qualifying the level of distortion introduced through the use of mismatched synthesizers for broadband conversion.

Figures 5.10 through 5.12 show the measured spectral response of the IF frequency for the self-mixing process when the $T_{x} / R_{x}$ synthesizer is driven by a wideband sinusoidal FM code using repetition rates of $1 \mathrm{KHz}$ and $10 \mathrm{KHz}$, and frequency deviations of $\pm 2.5 \mathrm{MHz}, \pm 625 \mathrm{KHz}$ and $\pm 156 \mathrm{KHz}$. Note here the wideband code used for testing is the sinusoidal test vector previously described in Figure 2.1 (a) (with no data added). Ideally a pure tone should result at $105 \mathrm{MHz}$. What is expected is some level of sideband distortion due to the difference in delays between $T_{x}$ and $R_{x}$ paths. The magnitude of the peak frequency deviation $\Delta f_{I F}$ is measured, and hence the effective maximum phase deviation $\beta_{I F}$ seen at the IF frequency. This can be used as an empirical measure of the quality of the best case broadband conversion process. Table 5.1 presents the measured results from this ideal case. It shou'd be noted that the results in Table 5.1 are the measured results for the $T_{x}$ synthesizer used as the $T_{x} / R_{x}$ synthesizer. The $R_{x}$ synthesizer results were virtually identical and therefore Table 5.1 and Figures 5.10 through 5.12 represent the measuring sensitivity of the test set-up for each combination of independant variables. Essentially the test set-up of Figure 5.19 can be viewed as a differential delay discriminator, similar to ones used in FSK demodulators. The difference between the received frequency and a delayed version of it, when 
TABLE 5. 1 : Ideol Broadband Conversion Results.

\begin{tabular}{|c|c|c|c|c|c|}
\hline \multicolumn{3}{|c|}{ Independant Variables } & \multicolumn{2}{|c|}{ Dependant Variables } & \multirow{2}{*}{ 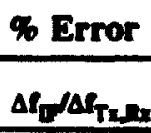 } \\
\hline$f_{m}$ & $\Delta f_{T_{x}, R_{x}}$ & $\boldsymbol{\beta}_{\mathrm{T} \mathbf{R}_{\mathbf{X}}}$ & $\Delta \mathbf{f}_{\mathrm{fF}}$ & $\boldsymbol{\beta}_{\mathbf{I P}}$ & \\
\hline \multirow[t]{3}{*}{$1 \mathrm{KHz}$} & $\pm 2.5 \mathrm{MHz}$ & 5000 & $2 \mathrm{KHz}$ & 2 & $0.04 \%$ \\
\hline & $\pm 625 \mathrm{KHz}$ & 1250 & $<2 \mathrm{KHz}$ & $<2$ & $\sim 0.04 \%$ \\
\hline & $\pm 156 \mathrm{KHz}$ & 312 & $<2 \mathrm{KHz}$ & $<2$ & $\sim 0.04 \%$ \\
\hline \multirow[t]{3}{*}{$10 \mathrm{KHz}$} & $\pm 2.5 \mathrm{MHz}$ & 500 & $20 \mathrm{KHz}$ & 2 & $0.4 \%$ \\
\hline & $\pm 625 \mathrm{KHz}$ & 125 & $<20 \mathrm{KHz}$ & $<2$ & $-0.4 \%$ \\
\hline & $\pm 156 \mathrm{KHz}$ & 31.2 & $<20 \mathrm{KHz}$ & $<2$ & $-0.4 \%$ \\
\hline
\end{tabular}

compared to a decision threshold level, can be used to determine whether a higher or lower frequency was transmitted. The value of the delay required in such systems is a function of the period of the received frequency. In this set-up the delay is fixed by the path between the $T_{x}$ synthesizer and the delayed version of itself through the offset frequency generation.

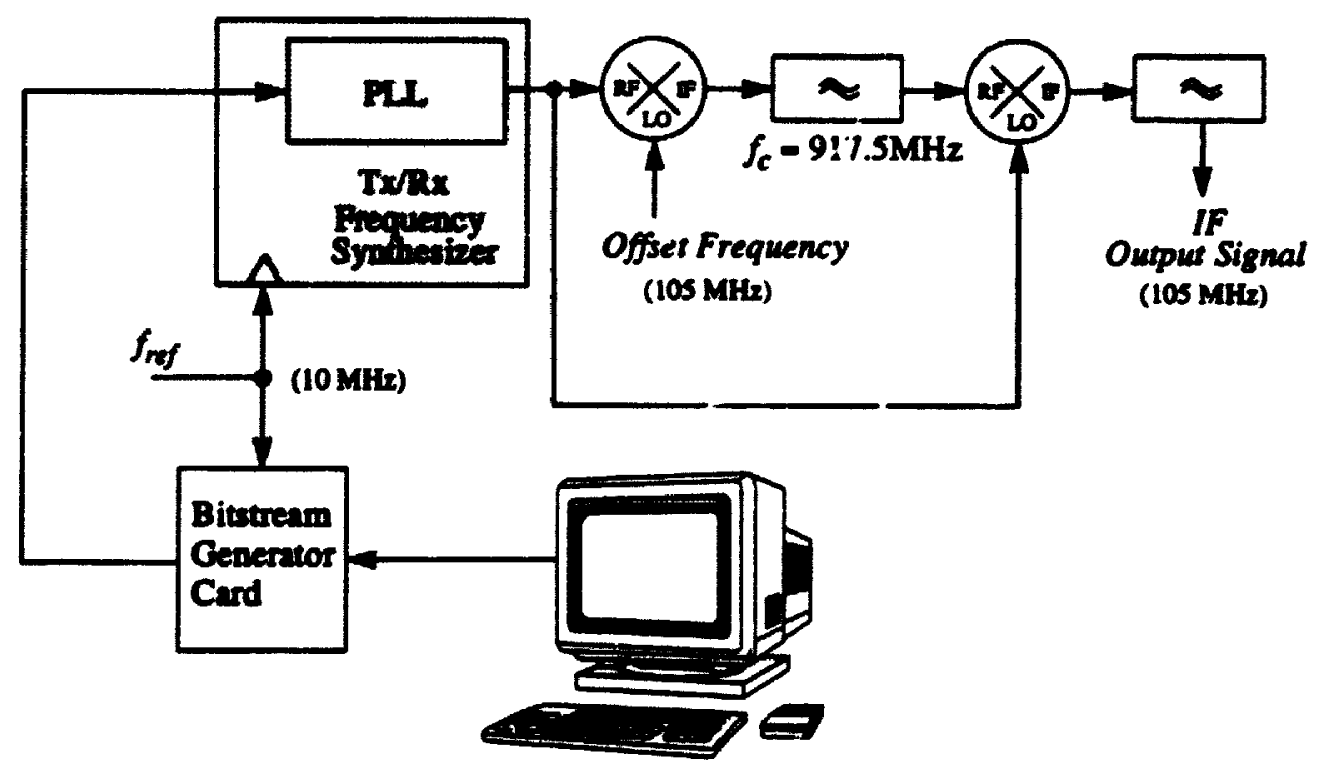

Figure 5.9 : Block diagram of the experimental testbed used for evaluation of ideal broadband conversion. 


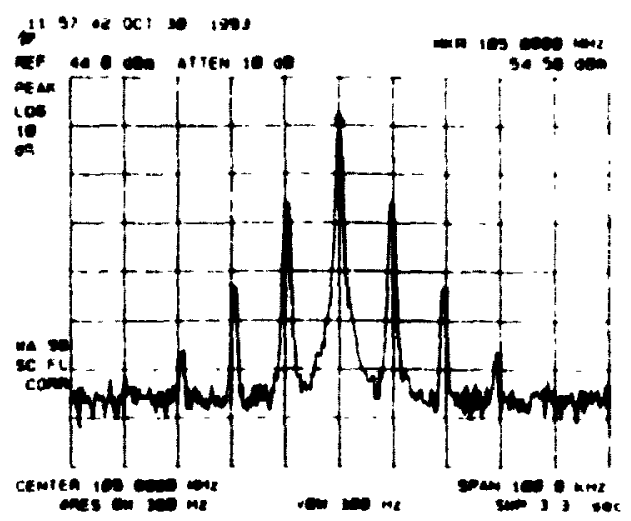

(a)

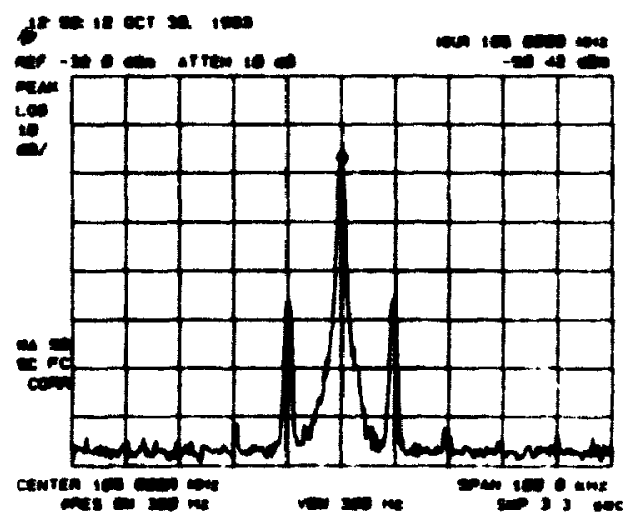

(b)

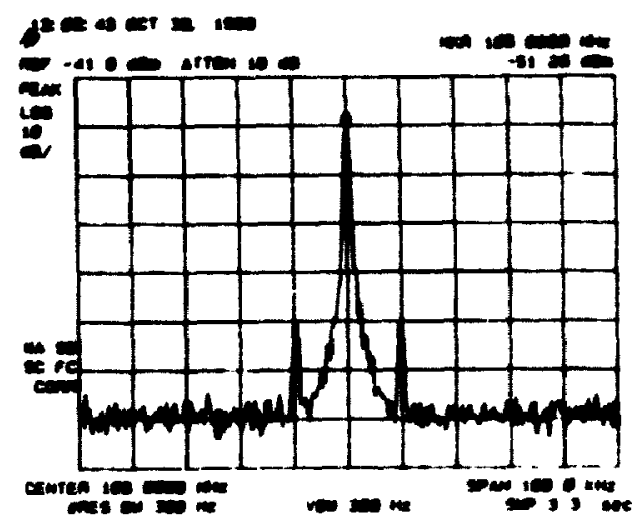

(c)

Figure 5.10 : Plots of the power spectrum of the $T_{Y}$ in self mixing for a frequency deviation of a) $\pm 2.5 \mathrm{MH}, 6) \pm 625 \mathrm{KHZ}$, and c) $\pm 156 \mathrm{KHz}$ at a nte of $10 \mathrm{KHz}$. 


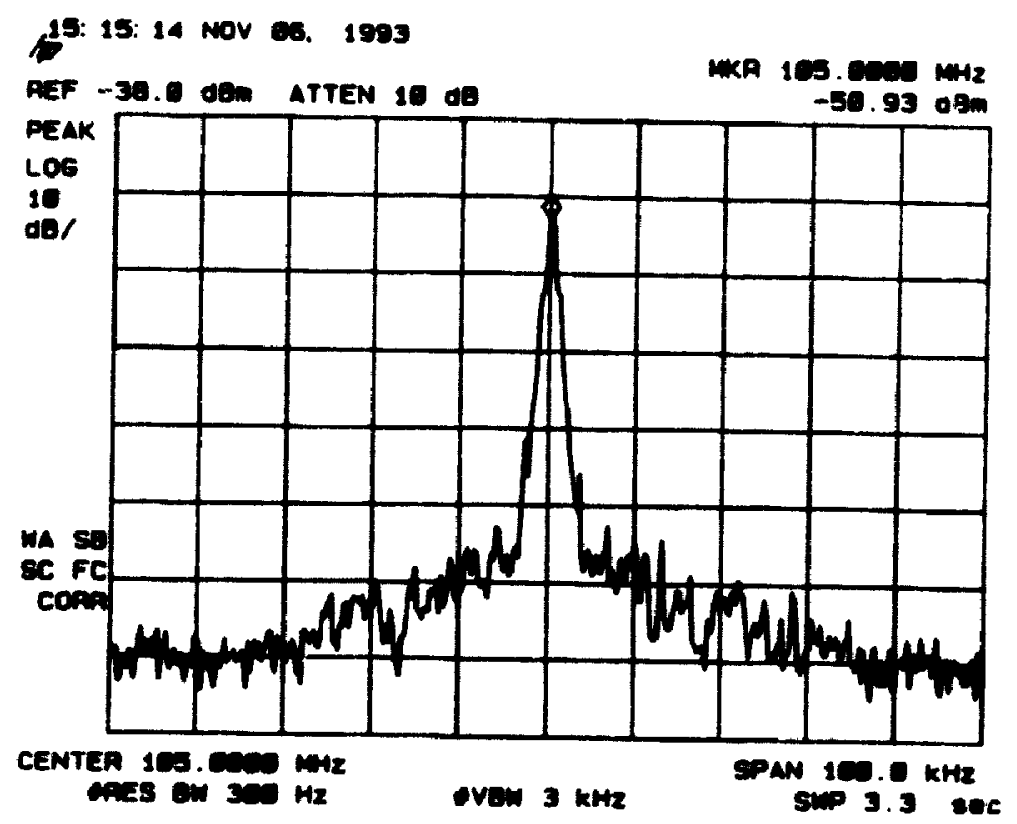

(a)

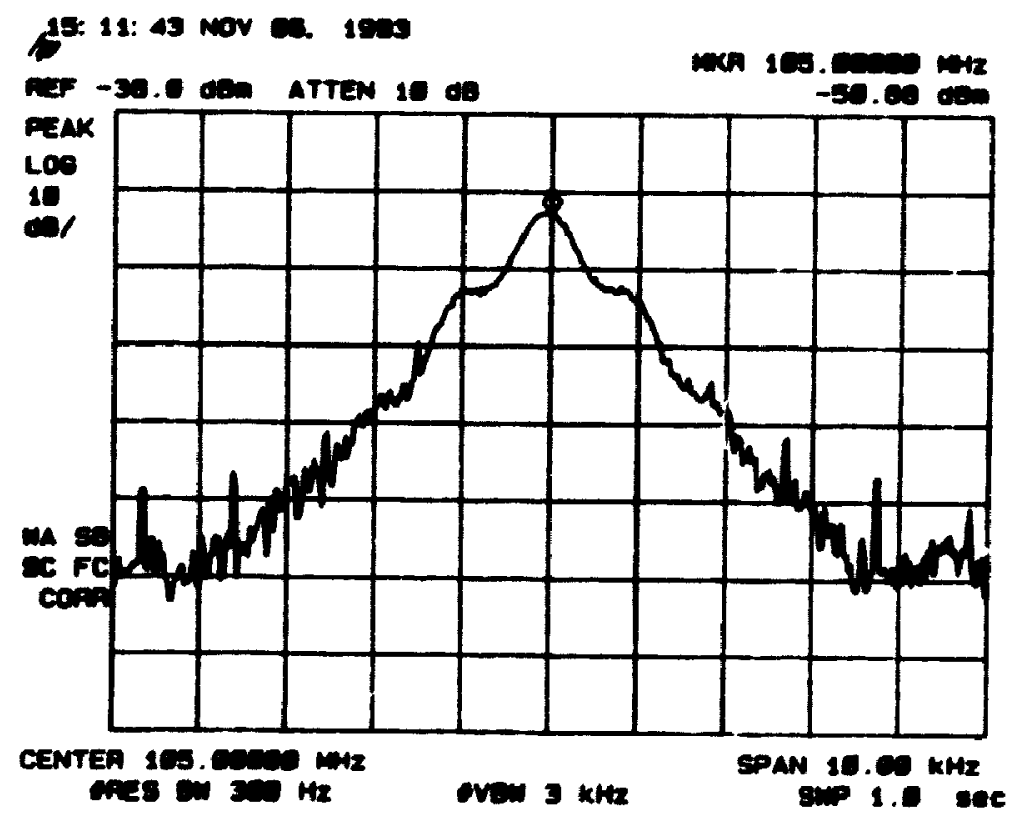

(b)

Figure 5.11 : Plots of the power spectrum of the $T_{x}$ in self mixing for a frequency deviation of $\pm 2.5 \mathrm{MHz}$ and a rate of $1 \mathrm{KHz}$ for wide and narrow frequency spans. 


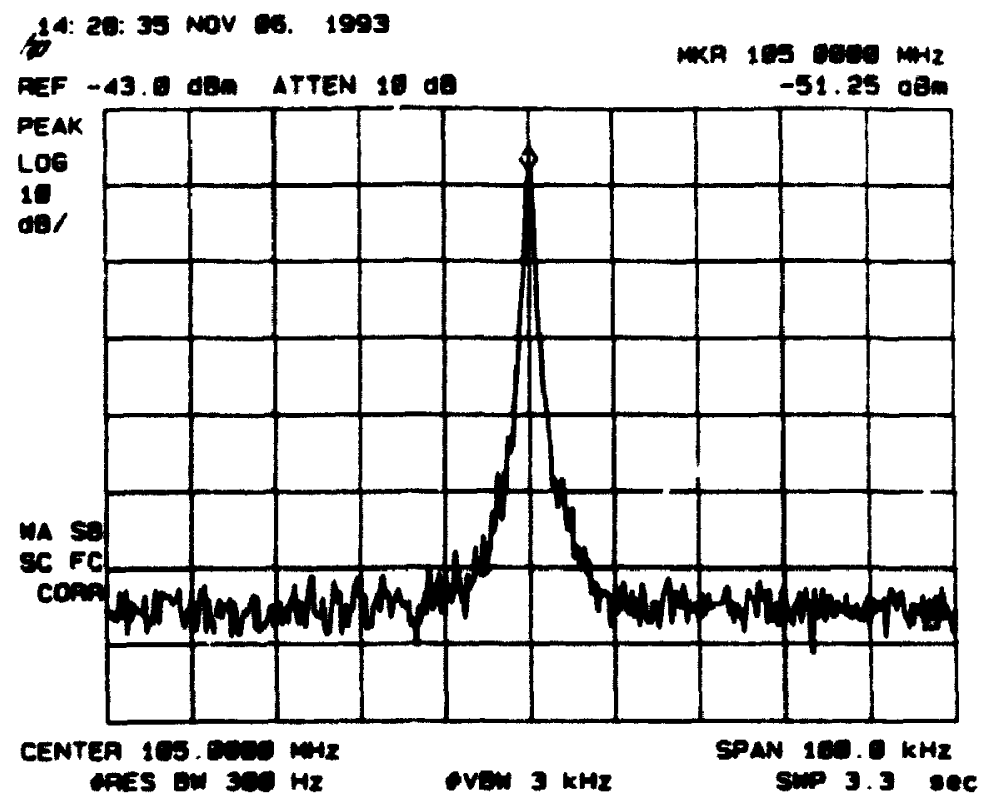

(a)

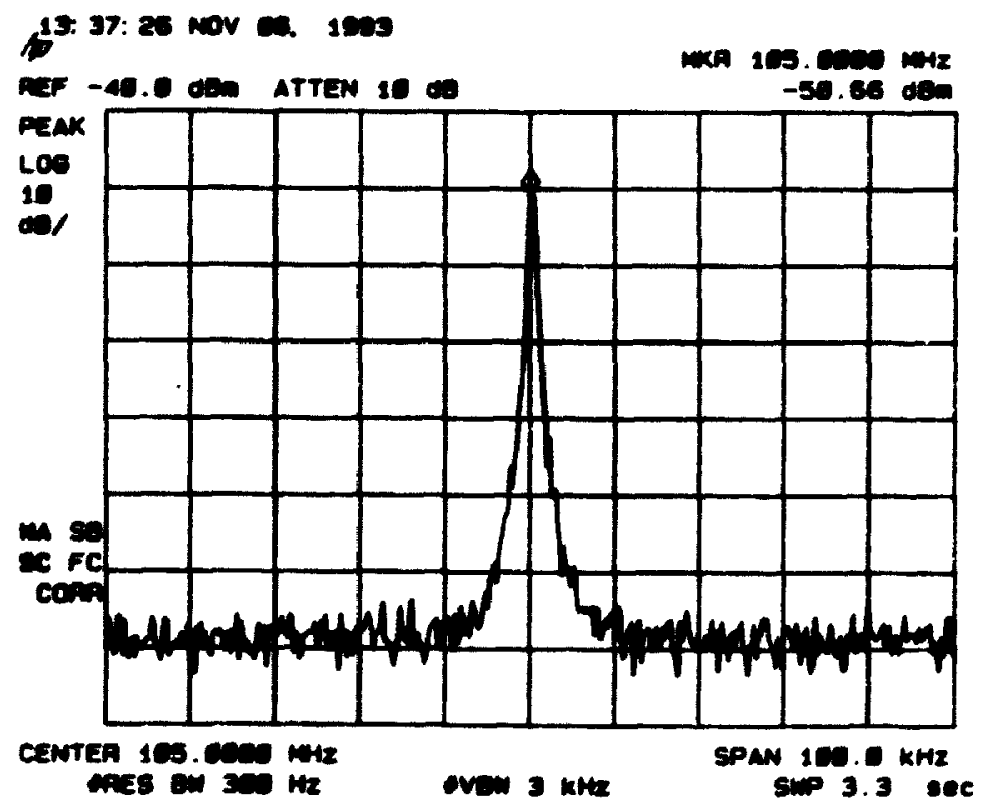

(b)

Figure 5.12 : Plots of the power spectrum of the $T_{x}$ in seif mixing for a frequency deviation of a) $\pm 625 \mathrm{KHz}$ and b) $\pm 156 \mathrm{KHz}$ at a rate of $1 \mathrm{KHz}$ 
Variations in the period of the $T_{x}$ signal are a result of changing the repetition rate of the broadband signal from $1 \mathrm{KHz}$ to $10 \mathrm{KHz}$.

The results of this broadband measurement clearly indicate that, aside from the effect of delay discrimination, wideband deviations as large as $\pm 2.5 \mathrm{MHz}$ for a 10 $\mathrm{KHz}$ repetition rate result in very little perceivable distortion when self mixed.

\subsection{Broadband Conversion Measurement}

If two separate synthesizers are now considered for the $T_{x}$ and $R_{x}$ components, Table 5.2, summarized from Figures 5.13 and 5.14, shows the results of the broadband conversion measurements, for the given sinusoidal FM peak frequency deviations and code repetition rates (no data present on the test vector).

TABLE 5.2 : Brondband conversion mesurement results.

\begin{tabular}{|c|c|c|c|c|c|}
\hline \multicolumn{3}{|c|}{ Independant Variables } & \multicolumn{2}{|c|}{ Dependant Varisbles } & \multirow{2}{*}{ 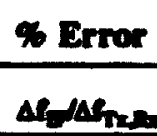 } \\
\hline 5 & $\Delta f_{\text {Trex }}$ & $\beta_{\text {Tring }}$ & $\Delta_{\mathbf{D}}$ & $\beta_{\mathbf{L}}$ & \\
\hline \multirow[t]{3}{*}{$1 \mathrm{KHz}$} & $\pm 2.5 \mathrm{MHz}$ & 5000 & $30 \mathrm{KHz}$ & 30 & $0.60 \%$ \\
\hline & $\pm 625 \mathrm{KHz}$ & 1250 & $8 \mathrm{KHz}$ & 8 & $0.64 \%$ \\
\hline & $\pm 156 \mathrm{KHz}$ & 312 & $2 \mathrm{KHz}$ & 2 & 0.648 \\
\hline \multirow[t]{3}{*}{$10 \mathrm{KHz}$} & $\pm 2.5 \mathrm{MHz}$ & 500 & $700 \mathrm{KHz}$ & 70 & $14.0 \%$ \\
\hline & $\pm 625 \mathrm{KHz}$ & 125 & $140 \mathrm{KHz}$ & 14 & $11.2 \%$ \\
\hline & $\pm 156 \mathrm{KHz}$ & 31.2 & $40 \mathrm{KHz}$ & 4 & $12.8 \%$ \\
\hline
\end{tabular}

The most obvious conclusion for the results of Table 5.2, considering the ideal results of Table 5.1, is that there are large mismatches between the PLL gains of the $T_{x}$ and $R_{x}$ frequency synthesizers. The ideal broadband conversion process provided evidence that broadband conversion works well for ideally matched synthesizers, regardless of peak deviation or repetition rate. This means that modulation rates approaching the bandwidth of the phase locked loop could be impiemented if both the open loop gain $H_{O L}$ and the transient response of the transmitting and receiving symthesizers were well matched. Although this is not recommended, the idea was successfully tested with a $\pm 2.5 \mathrm{MHz}$ sinusoidal test vector with a $50 \mathrm{KHz}$ repetition rate using the ideal conversion process (self 
mixing) of Figure 5.9. The results of this specific test will not be presented in this thesis.

The results of Table 5.2 show that changes in peak deviation $\Delta f_{T x, R x}$ result in an IF peak phase deviation error that is roughly a fixed percentage of $\Delta f_{T_{x}, R x}$. On the other hand changes in the repetition rate result in an error that is a function of frequency (ie; phase error suppression increases with decreasing frequency). Both of these observations are supported by the theory of linear time-invariant systems [Oga70]. What is crucial about these results is the significant levels of IF distortion that have resulted from mismatches in the PLL open loop gain between the $T_{x}$ and $R_{x}$ frequency synthesizers and the delays between the synthesiers in the test setup (the latter being non-dominant;. Equation 5.13 provides an approximate theoretical closed form solution which suggests the source of the mismatch between ihe $T_{x}$ and $R_{x}$ synthesizers. If a deviation of $\pm 156 \mathrm{KHz}$ and a repetition rate of $1 \mathrm{KHIz}$ is considered as an example, then from Table 5.2, $\beta_{I F}$ is measured to be 2 . If these two values are now applied to equation 5.13, and $H_{O L, R X}$ is assumed to be $20, H_{O L, T X}$ as $30, \theta_{R X, e}$ as -30 degrees, and $\theta_{T X, e}$ as -10 degrees, then the calculated magnitude of the residual phase deviation $\beta_{r}$ is found to be 4.99. This is well within a magnitude of the measured value.

A qualitative evaluation of SNR based upon these results indicates that the frequency deviation required to transmit a data bit (over the level of mismatch distortion) in order to make the data bit recoverable increases in direct proportion to the FM repetition rate. It should be noted that the level of SNR determined from the measured results would not include real channel distortion. However, real channel distortion would be non-dominant with respect to the distortion levels that have resulted from synthesizer PLL mismatch, for the particular synthesizers used here.

The disparity between the $T_{x}$ and $R_{x}$ synthesizers finds its source in the design of their type 2 fifth order phase locked loops. Initially the loops were designed with static LO operation in mind, and therefore additional filtering was introduced to reduce phase noise levels and reference frequency feedthrough. Yet the fifth onder design consists of many independant variables (time constants) that affect the gain and delay response of the loop, as shown in the detailed design of section 3.1 in this thesis. Trying to match the open loop gain in a differential environment with discrete components that all have a $10 \%$ tolerance has led to differences in the $T_{x}$ and $\boldsymbol{R}_{\boldsymbol{x}}$ loops that appear to difficult to resolve in the present prototypes. It should 


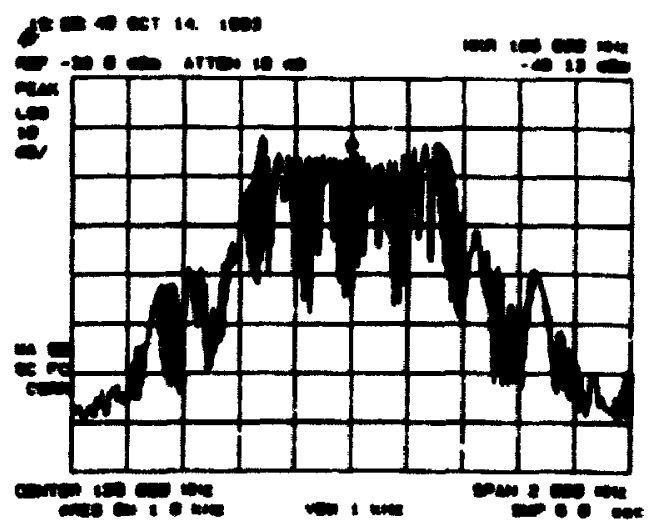

(a)

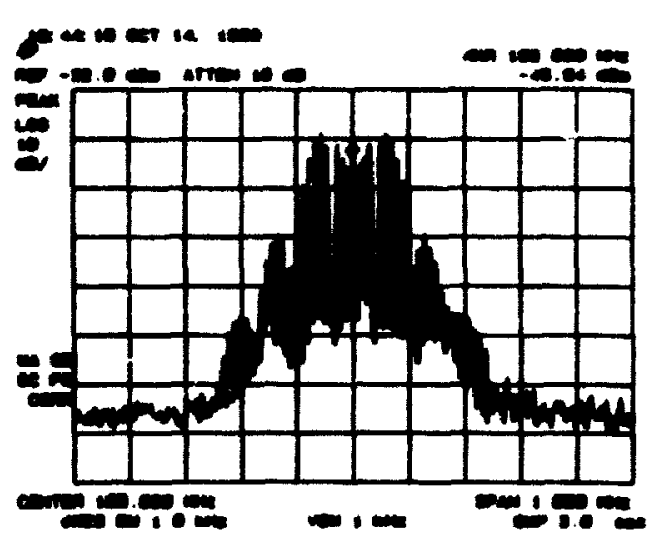

(b)

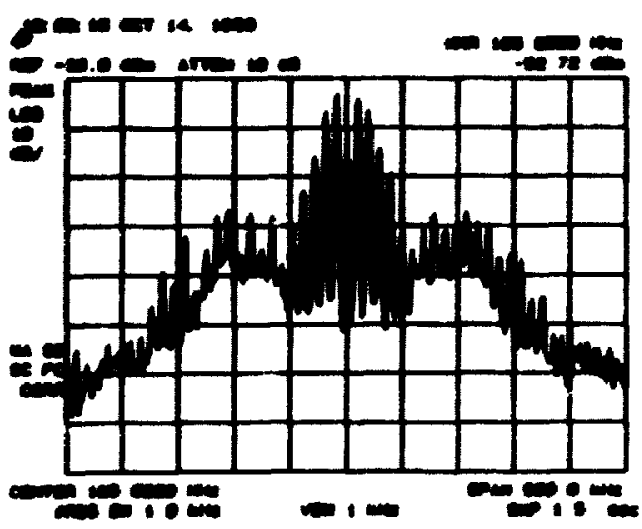

(c)

Figure 5.13 : Plots of power gpectrum for the IF frequency uping a $10 \mathrm{KHz}$ repetition rate and peak deviations of a) $\pm 2.5 \mathrm{MHz}$, b) $\pm 625 \mathrm{KHz}$, and c) $\pm 156 \mathrm{KHz}$. 


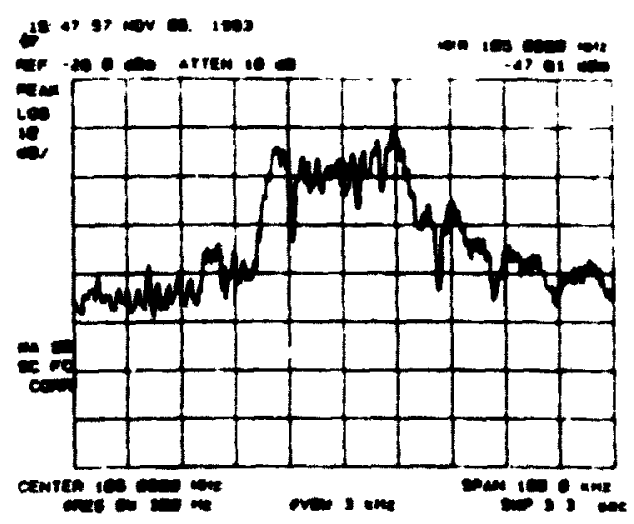

(a)

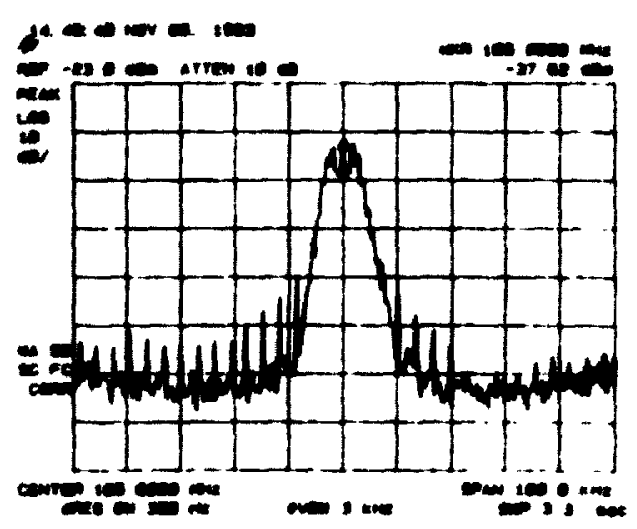

(b)

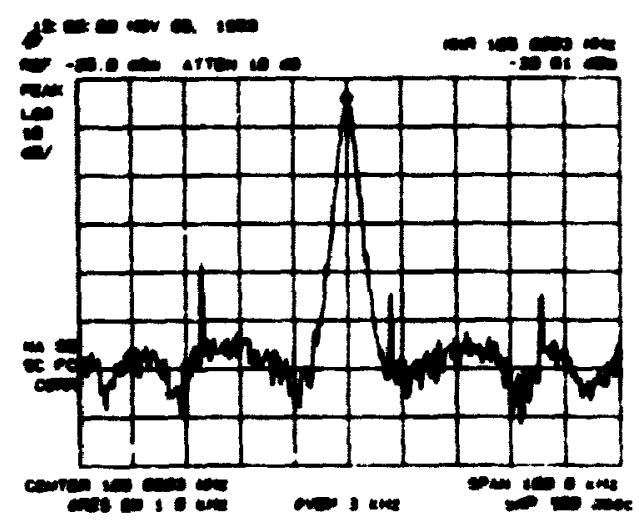

(c)

Figure 5.14 : Plots of power spectrum for the If frequency using a $1 \mathrm{KHz}$ repetition rate and peak deviations of a) $\pm 2.5 \mathrm{MHz}$, b) $\pm 625 \mathrm{KHz}$, and c) $\pm 156 \mathrm{KHz}$. 
be noted that integrated circuit implementation of the synthesizer are being designed to have considerably higher bandwidths and hence less susceptability to the problems encountered in the prototypes. In the prototypes, sensitivity to PCB layout due to parasitics, ground plane design, and magnetic cross-coupling also affected the final results. These types of effects are characteristic of a PCB design but not representative of an IC implementation.

An attempt was made to provide tuneability to the overall loop gain by placing a DC clamping circuit (ie; a voltage divider implemented using a trimpot) on the tuning voltage of the VCO of each synthesizer. Independant scaling of the $T_{x}$ and $\boldsymbol{R}_{x}$ loop gains provided a technique for empirically minimizing loop gain mismatches. Yet this brute force approach was incapable of tine tuning the independant time delay gain elements of each loop. The minimizing techniquc was applied each time a test vector was changed and the use of this gain scaling, through the clamping circuit, provided clear evidence that increasing loop gain had the effect of reducing the $T_{x}$ and $R_{x}$ synthesizer mismatch. The limitation of the simple clamping circuit lay in the fact that magnitude of all the delay elements around were only scaled by a fixed percent and not tuned independently. The results of Table 5.2 were measured when the differences between the $T_{x}$ and $R_{x}$ synthesizers were minimized using the clamping circuit. Therefore the most immediate work that must be done as a next step in investigating the viability of broadband coded FM for wireless communications is exploration of the capability of precisely matching the gains of a set of $T_{x}$ and $R_{x}$ frequency synthesizers that will operate with a similar resolution but with a fixed frequency offset. This will require the paring down of the order of the present PLL synthesizer prototypes to reduce the number of matching variables, and then an investigation of how the altered levels of phase noise will affect the SNR of the receiver and/or the radio design, based upon the allocated bandwidth of the wideband channel.

\subsection{Qualifying the IF Signal for $\Delta \Sigma$ Frequency Discrimination}

In earlier discussions, transceiver operation was based upon the premise that an IF frequency discriminator was available and could be cascaded to the output of the IF mixer. It was unfeasible at this point to test the overall broadband transceiver structure because of the performance limitations at the present state of the research. However, this section makes an attempt to qualify some of the necessary 


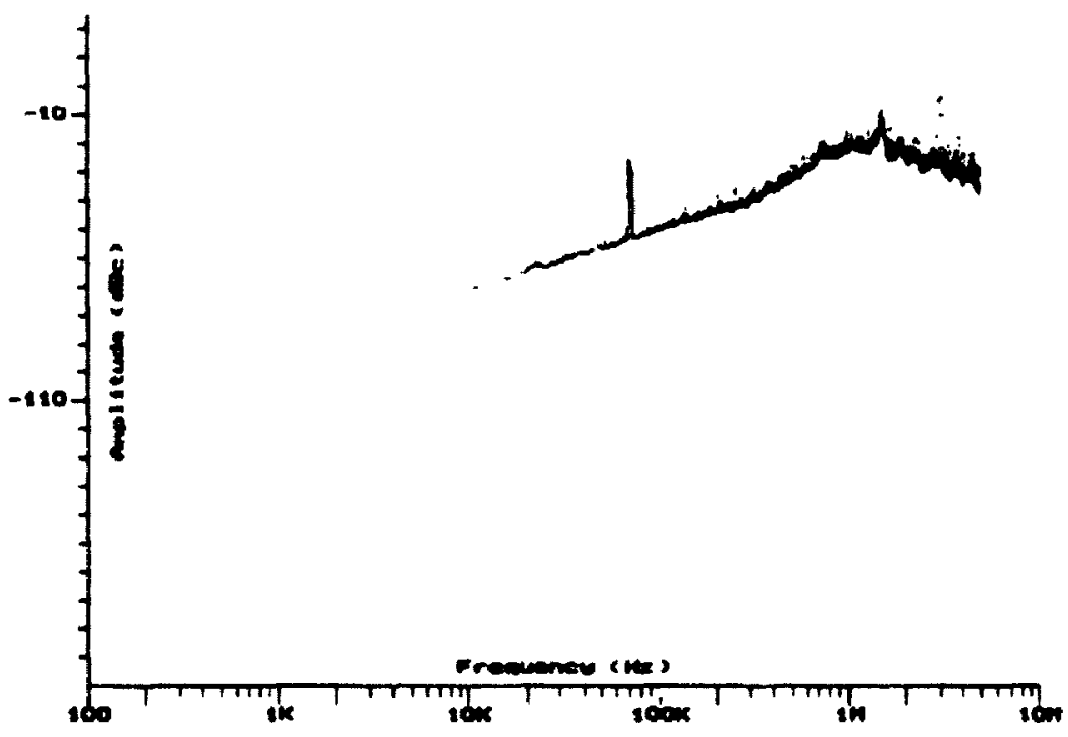

Figure 5.15: The power spectral density of the captured discriminato* output bitstream.

interface structure required if the $\Delta \Sigma$ frequency discriminator were to be cascaded to the IF output.

From Chapter 4 , measured results on the $\Delta \Sigma$ frequency discriminator indicated that its operation was suited to low data rate, wide frequency deviation signals in order to provide adequate SNR at its output. This finding comes from the use of a printed circuit board (PCB) prototype operating with a $10 \mathrm{MHz}$ sampling rate $\left(f_{\text {ref }}\right)$ on a sinusoidal FM modulation signal of $f_{m}=70 \mathrm{KHz}$ and $\Delta f= \pm 50 \mathrm{KHz}$. Figure 5.15 (reprinted from Figure 4.13) shows the power spectral density of the oversampled bitstream. The magnitude of the $70 \mathrm{KHz}$ modulation tone in the spectrum is directly proportional to the peak deviation $\Delta f$ of the sinusoidal input. A deviation of $\pm 50 \mathrm{KHz}$ produced a SNR of only $\sim 8 \mathrm{~dB}$. In order to realize increased levels of SNR then the peak deviation $\Delta f$ must also be increased. The constraint of low data rates on the discriminator input is a function of the order of the $\Delta \Sigma$ modulator and the sampling rate. Figure 5.15 results show only first order noise shaping for a second order design. The impairment in the noise shaping reduced the effective dynamic range of the digital signal. if second order noise shaping had been present greater levels of SNR would have resulted through the reduction of the quantization noise floor. 


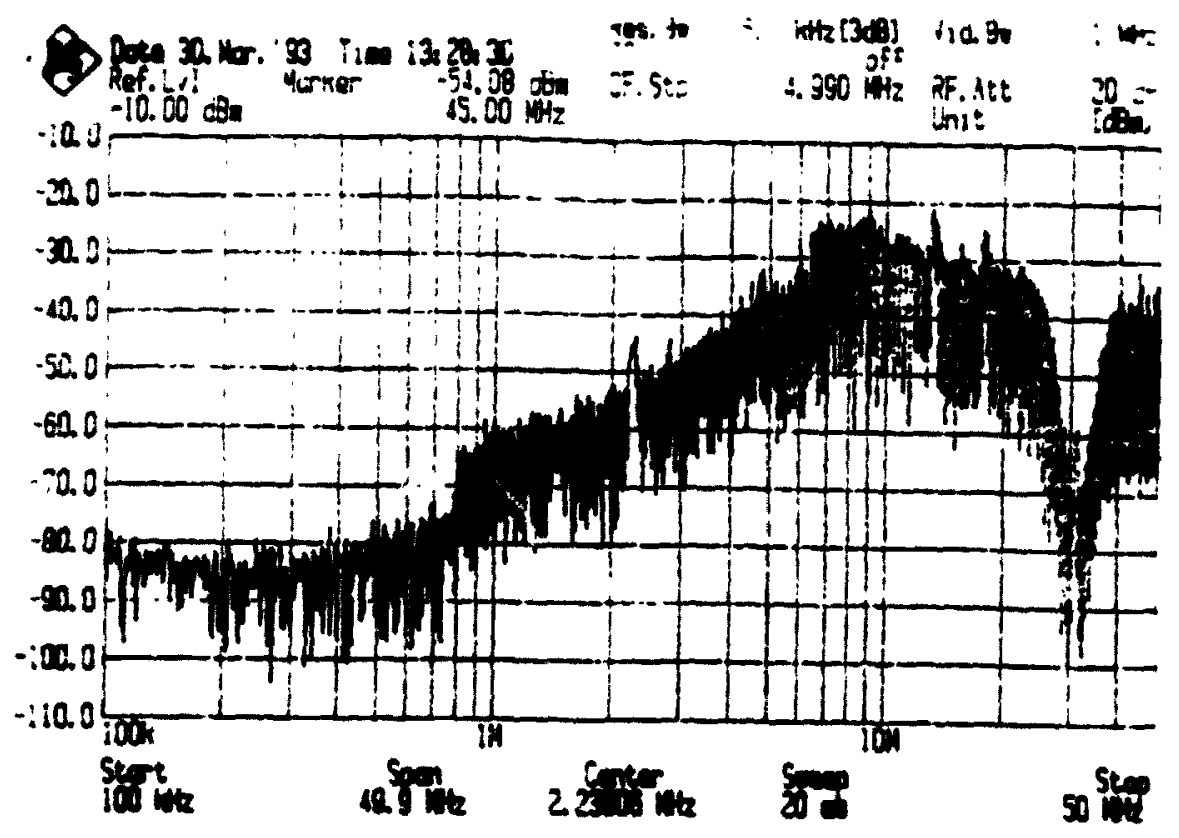

Figure 5.16 : Power spectral density of a $\Delta \mathbf{\Sigma}$ frequency discriminator bitstream output, reported by Beards and Copeland [BC92].

Although the results of Chapter 4 indicate poor performance for the printed circuit board protorype (ie; due to charge pump leakage), there is an example of a working VLSI monolithic implementation with much better performance and wider bandwidths [BC92], as indicated in Figure 5.16. If such performance can be assumed. then a radio receiver based upon the architecture of Figure 2.3 becomes more feasible.

The issue of power levels required to drive the frequensy discriminator is one that has not been dealt with yet. From Figure 4.10, the discriminator input is the clock input on the dual modulus divider DMD. Data sheets on the divider used, SP9680 [Ples88], specify peak to peak input voltage levels of between 0.4 to 0.8 volts should be supplied to the DMD input. The SP8680 DMD is a 10K compatable ECL component, therefore interfacing a high speed, low level signal requires the use of amplification, ac coupling, and level shifting through a 508 matching circuit.

Obviously a purpose-designed DMD would be required in practice that would function with lower input levels (ie; smaller signal swings). Figure 5.17 shows a block diagram of the broadband conversion set-up along with the signal levels at 


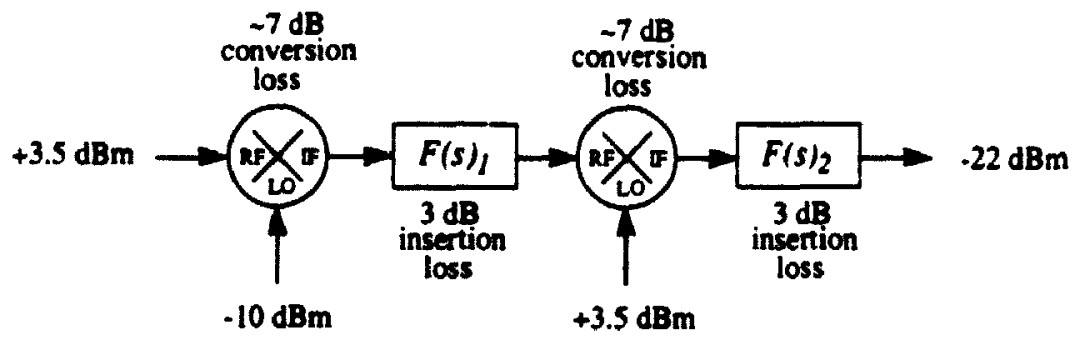

Figure 5.20 : Block diagram of the broadband conversion set-up including the VO signal levels.

the V/O ports. The mixers used in the test setup of Figure 5.1, ZLW-2 [MCI], specified a nominal LO input power of $+7 \mathrm{dBm}$ and an $R F$ input power of $+1 \mathrm{dBm}$. The actual power levels used in the test setup, shown in Figure 5.17, deviated from the recommended power levels resulting only in increased levels of conversion loss [MCI]. The level of $-22 \mathrm{dBm}$ into $50 \Omega$ provides a peak to peak voltage of $0.355 \mathrm{mv}$, which could not drive the DMD clock input. As previously mentioned, the DMD input is an ECL component and consists of either an emitter follower input circuit or emitter coupled pair input circuit. This implies a power level with 0.4 to 0.8 volts peak to peak signal swing at current levels capable of driving the base of a bipolar transistor. If the broadband conversion and $\Delta \Sigma$ frequency discriminator circuits were connected together in a receiver configuration, then an amplifier would have to match and amplify the filter $F(s)_{2}$ output to provide the correct signal levels to drive the DMD input. Alternatively, active mixers with conversion gain could be used. Tuning the gain wo!ld help to compensate for losses in the passive filters and allow the provision for optimal signal swing.

In this thesis the $\Delta \Sigma$ frequency discriminatur was not connected to the output of the IF stage of the broadband conversion test setup because the performance of the initial breadboard was not up to the standard that would be required. In addition. the $10 \mathrm{MHz}$ reference clock was not available and could not be derived from the reference clocks in use. The derived clock would have needed a DC phase offset function similar to that present on the HP3322B function generators in order to align the sampling frequency of the $\Delta \Sigma$ frequency discriminator so that it sampled after a valid setup time on the clock input to the DMD. This situation impeded an academic evaluation of the combination of the receiver and $\Delta \mathbf{\Sigma}$ frequency discriminator as a radio front end. 'low ever, knowing the capability of the receiver 
to the IF stage and the SNR of the $\Delta \Sigma$ frequency discriminator provided enough irformation to qualify them as suitable candidates for a fully integrated (ie; small number of external passive components) digital radio receiver. Research work in progress (N. Filiol) will carry this further. 


\section{Chapter 6}

\section{Conclusions and Recommendations}

\subsection{Conclusions and Summary}

The growing number of users of digital radio services has led to the demand for new and novel architectures that meet application specific requirements in a monolithic environmert in both an efficient and economical manner.

The individual contribution of this thesis was the evolution of the higher level radio design and test concept for the proposal of the broadband coded FM application. Contributions also included support work for the evolution of the two components that were investigated as the building blocks for the transceiver, as proposed in Figure 6.1, with synchronizat un for the receiver as proposed in Section 2.2.2, Figure 2.5. The components were tested separately and in the broadband FM context using a broadband conversion test set-up. The broadband conversion concept, a continuous frequency varying modulation where $\Delta f$ approaches an infinitesimally small increment (double precision interpolation) in the limit, was tested under both ideally matched synthesizer PLL open loop gain parameters, and poorly matched gain parameters. The results provided insight into the practical requirements for implementing broadband coded FM as an alternative technique for combating the effects of multipath fading in an indoor wireless environment. The results and testbed components additionally provide a foundation for further systems level research into similar areas of broadband modulation for digital radio.

Initially a printed circuit board prototype of a $\Delta \Sigma$ modulated fractional- $N$ frequency synthesizer was constructed and tested in the $800 \mathrm{MHz}$ frequency range, based upon a $10 \mathrm{MHz}$ reference frequency and a type II fifth order PLL. In fixed frequency operation, phase noise levels of $-100 \mathrm{dBc} / \mathrm{Hz} @ 1 \mathrm{KHz}$ were achieved, while in fractional-N division operation results degraded slightly, as expected, to $90 \mathrm{dBc} / \mathrm{Hz} @ 1 \mathrm{KHz}$. Transient behavior to frequency steps showed settling times 


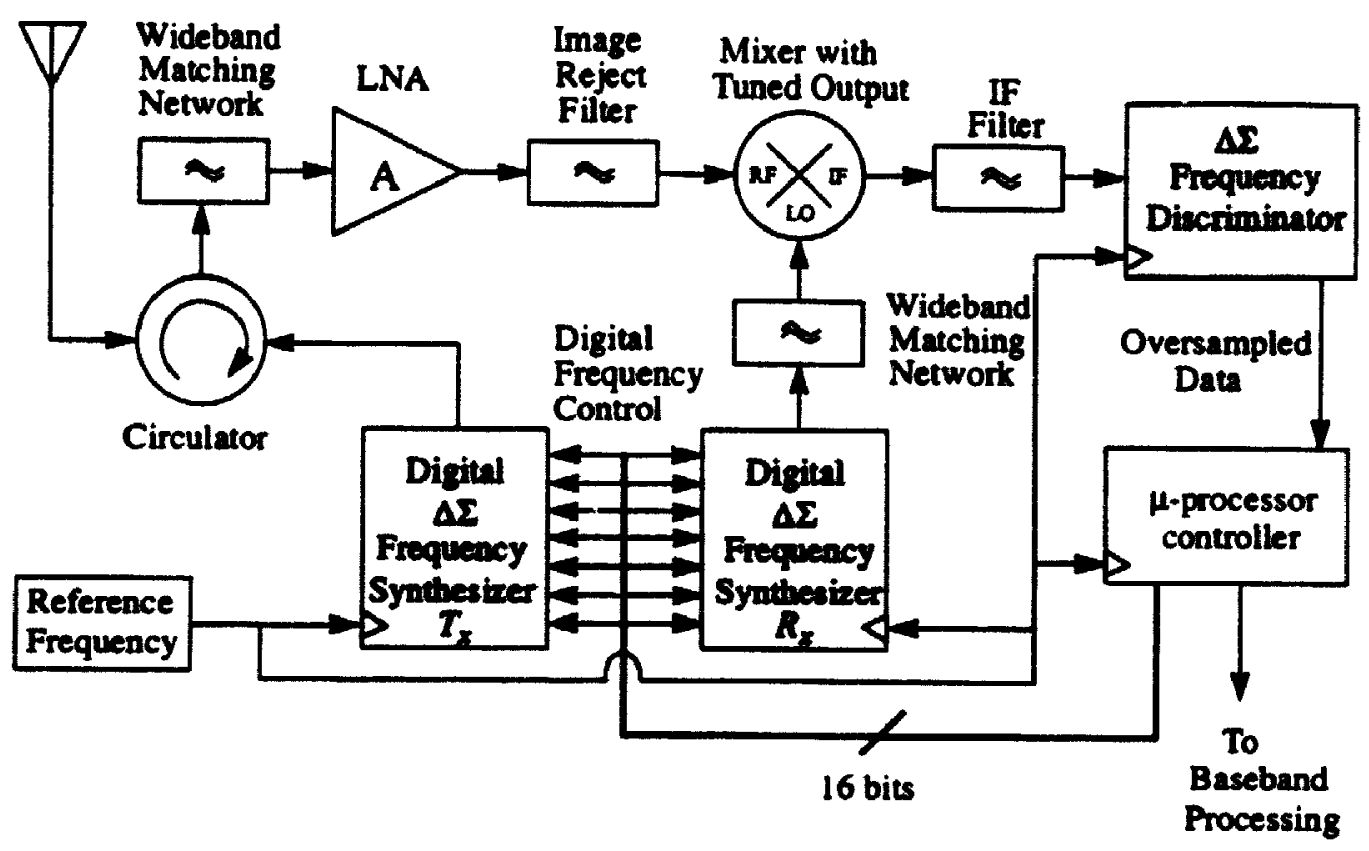

Figure 6.1 : Block diagram of a $\Delta \Sigma$ Digital FM transceiver.

on the order of $30 \mu$ for a $20 \mathrm{MHz}$ frequency step. Measurement of frequency resolution indicated capabilities of $1 \mathrm{~Hz}$ movement by toggling the fifth LSB of the $\Delta \Sigma$ modulator input. Typically this type of synthesizer is suited to low data rates as a transmitter due tu the limitations of the PLL bandwidth, and is therefore ideally suited to low data rate wide deviation FM applications. Work on evolving monolithic versions are presently in progress using both CMOS and BiCMOS processes [Th93, RCK93].

A printed circuit board prototype of a $\Delta \Sigma$ frequency discriminator was also constructed and tested for a $105 \mathrm{MHz}$ input center frequency, and a $10 \mathrm{MHz}$ sampling frequency. Results for an analog sinusoidal FM input, using a data rate of $50 \mathrm{KHz}$ and a peak deviation of $\pm 50 \mathrm{KHz}$, produced output signal-to-noise levels of approximately $9 \mathrm{~dB}$. This low value was the result of an impairment in the noise shaping function of the discriminator. Only first order noise shaping was achieved in the initial design. The degradation in operation was a result of charge leakage from the integrating capacitors of the charge pump circuit. Although the results of the printed circuit board version were not fully adequate for the application, the concept was proven ard provided a level of confidence to the innovators (Beards and Copeland) that the VLSI implementation would meet with success. At the time 
of this writing initial results for a VLSI implementation were very encouraging, as seen in Figure 5.25. The circuit optimization and SNR expectations for the baseband signal of such a discriminator are presently under investigation by Beards [Bea94].

The results of Chapter 4 indicated that the prototype frequency discriminator was well suited to low data rate high deviation FM applications. The low data rates place the modulation tone further down into baseband where the levels of the shaped quantization noise are non-dominant. The use of wide deviation FM enhances the SNR of the discriminator output signal through the direct relationship between the RF FM peak deviation and the IF peak signal strength.

The availability of two digitally controlled circuit board frequency synthesizer prototypes, one to act as a transmitter and the other for use as a receiver, afforded the opportunity to reach beyond the simple technique of heterodyne conversion and allowed testing of a novel broadband mixing concept. Testing was accomplished using a double precision digitally encoded sinusoidal FM test vector. The IF signal characteristics were measured as a function of the sinusoidal FM repetiticn rate and peak frequency deviation (no data present on the test vector). The test vectors were varied over a frequency range similar to one that would result from the use of a true pseudorandom broadband code. The results from the experimental testbed evaluation showed that for ideally matched frequency synthesizers, less than $1 \%$ error occurred at IF frequencies (an artifact of delay discrimination) regardless of the magnitude frequency deviation. For unmatched frequen:y synthesizers operating with wide deviation high rate FM signals, the level of mismatch in phase error suppression (ie. a function of open loop gain) led to as much as $15 \%$ error at IF frequencies for $10 \mathrm{KHz}$ repetition rates. For practical application this would require that data deviations on the pseudorandom code be greater than 50\% of the total error at the IF band, in order to provide the minimum margin necessary in order to detect the presence of a one or zero data bit. Such data deviations would be impossible for present day digital wireless standards (ie; $\mathrm{CT2t}$ ) due to the bandwidth restrictions (ie; transient behavior) of the synthesizer PLL.

In the test environment, variations in the repetition rate were kept well within the loop bandwidth of the PLL to ensure that frequency mismatch between synthesizers was not a result of differences in the overshoot and settling time of the PLLs. For practical purposes this limited the data rates in the prototype 
synthesizers to about $40 \mathrm{KHz}$, due the $20-25 \mu \mathrm{sec}$ settling time of the loop. For more practical data rates the loop bandwidths would have to be opened wider to provide faster settling times. Testing is presently under way in further thesis work (N. Filiol) to properly characterize the broadband concept with suitable precision pseudorandom noise PN code sequence lengths, data rates and deviations to provide optimum SNR at the IF stage [Fi94]. Design of the PN codes based upon the coherence bandwidth for the frequency selective fading indoor wireless environment, and chosen for minimum cross correlation (ie; Gold codes) will lead to greater levels of SNR by combatting the effects of multi-path fading.

If the transceiver block diagram of Figure 6.1 is considered, then experimental results indicate that matching of the $T_{x}$ and $R_{x}$ synthesizer open loop gains $H_{O L}$ and phase and frequency response characteristics are fundamental for a broadband FM implementation. It may be possible to use a conventional zero crossing discriminator in the transceiver design, but the $\Delta \mathbf{\Sigma}$ frequency synthesizer and $\Delta \mathbf{\Sigma}$ frequency discriminator are duals (ie; one generates frequencies form $\Delta \Sigma$ bitstreams, and the other generates $\Delta \Sigma$ bitstreams from frequencies) and therefore complement one another. Both components are suitable for VLSI integration and are easily manipulated to run from the same reference frequency. When combined as shown in Figure 6.1 they are well matched for a wide deviation, low data rate broadband FM transceiver. Yet a transceiver based upon broadband conversion and the concepts presented in this thesis would not be well suited to applications where the $T_{x}$ and $R_{x}$ synthesizers were constructed with independant design specifications. The synthesizers must be well matched in both frequency and time domain responses. As a result the concept is better suited to cordless phone applications, especially for multi-channel indoor wireless PBX and conferencing switches. The use of digitally controlled broadband techniques, as outlined in this thesis, alleviates the requirement for a carrier recovery system, and provides a method for a less complex more fully integrated digital radio transceiver.

\subsection{Recommendations for Future work on the $\Delta \Sigma$ Fractional-N PLL Frequency Synthesizer}

The results for the printed circuit board version of the $\Delta \Sigma$ PLL frequency synthesizer were very promising. Yet the component count was very large, and therefore not very appealing as a printed circuit board product as distinct from a monolithic implementation. The onerous architecture could be reduced in several 
ways. First the DMD and MEC circuit could be combined resulting in a single component multi-modulus divider. This would allow a reduction in the gate count of the $\Delta \Sigma$ modulator by removing the concentrator section of the modulator. The complete modulator consisted of two seperate third order $\Delta \Sigma$ structures. The Lee and Sodini section [CNL90] was used to concentrate a four bit word to a single bit bitstream output. If the concentrator was removed from the circuit and the DMD designed as a multi-modulus divider, then the entire synthesizer architecture could be reduced by about 20 percent. The cost/benefit strategy of such a design would be more appealing for a VLSI implementation.

For non-static operation of the synthesizer, as in a broadband mixing application. the order of the PLL in the synthesizer has to be reduced. The requirement for well matched synthesizers limits the number of variables that can be introduced into the loop filter because the resulting complex filter response is very difficult to reproduce. Therefore for further broadband testing the loop order must be reduced from a fifth to a second order active loop filter. This will result in higher levels of reference frequency feedthrough and higher levels of double sideband phase noise. The phase noise issue will be fundamental only if the levels are significant enough to impair low end dynamic range and sensitivity, thereby impeding the reception of highly attenuated/faded signals The reference frequency feedthrough could be ignored because future designs could be based upon higher reference clocks (ie; such as in high speed VLSI implementations) which would experience greater suppression for the same loop filter order and bandwidth.

Research by Bax [Bax94] has led to work in the development of an arithmetically locked loop (ALL) as a spinoff from the $\triangle \Sigma$ PLL frequency synthesizer. The loop can provide digital calculation and control of loop parameters, which would allow a better solution for the matching problems raised in this thesis. The architecture results in a synthesizer with greater potential for monolithic implementation.

Work by Kwasniewski and Thamsirianunt [Th93] has provided promising results towards the development of a CMOS PLL frequency synthesizer (excluding loop filter) that is suitable for $\mathrm{GHz}$ range operation. This provides the first step in an initiative towards an all CMOS fully programmable radio transceiver.

One other area that still needs to be investigated for a monolithic fractional-N PLL synthesizer is a fully integrated CMOS $\Delta \Sigma$ modulator. The present version, implemented in an FPGA, suffers with degraded states for DC inputs and only provides a functional bitstream density for a range of 0.25 through 0.75 . The 
present version needs to have the concentrator circuit removed. and further work done on the MASH3 structure to provide a $\Delta \Sigma$ modulator with higher order noise shaping. It is possible that dithering concepts could be intoduced into the architecture to overcome the $D C$ input impairment. This would make a $\Delta \Sigma$ based PLL frequency synthesizer more suitable as a fixed frequency local oscillator in a radio receiver.

\subsection{Recommendations for Future work on the $\Delta \Sigma$ Frequency Discriminator}

The discriminator design implemented in this thesis was not well suited to printed circuit board techniques. The magnitude of the parasitic capacitive elements associated with transmission lines and soldering pads were well within the magnitude of the charge pump integrating capacitors, making it difficult to use the integrating capacitors on the charge pump as effective tuning elements.

The charge pump leakage current was also a problem that had no apparent solution in the printed circuit board environment. The 2N3904 surface mount transistor arrays suffered from Early voltage problems $\left(V_{A}\right)$ which resulted in a transistor output conductance that allowed current leakage from the integrating capacitors at a rate that affected the second order statistics of the discriminator. More work needs to be done in developing an architecture that is not so dependent on circuit problems in the analog integrator. Presently work has begun on such an architecture and has proceeded past a first iteration in the design cycle [Bax94].

A monolithic implementation of the discriminator by Beards [BC92], based upon his novel design, has provided a clear indication that the technique can be used successfully as a demodulation component in a radio receiver. What still needs to be done is a SNR analysis to show whether this technique, although well suited to VLSI implementation, will provide practical levels of SNR with respect to circuit techniques presently in use.

\subsection{Recommendations for Future work on a Digital FM Transceiver for Broadband Applications}

As indicated by the results of Chapter 5, more work needs to be done in characterizing the broadband concept with a suitably long enough code sequence 
length to determine an analy 'ic solution for designing a broadband transceiver for specified modulation rates and bandwidths.

The block diagram of Figure 2.5 presented a proposal for a broadband corted FM receiver. There is still a large amount of work to be done in implementing the clock recovery and synchronization network required to make the architecture practical. Filiol [Fi94] has begun work in this area.

Other areas discusser but not addressed in this thesis were the issues of noise figure, linearity, compression, dynamic range, and bandlimiting of the RF front end components ( $i e ;$ the LNA, mixer, and image filter). Although not fundamental to the work in this thesis, they are essential to any practical monolithic digital radio implementation. Analytical issues and novel BiCMOS integrated designs for both an LNA and double oalanced mixer are presently under investigation by Long and Copeland [Lon94] and may provide solutions for an integrated analog front end. Image reject filtering, based upon the use of planar on-chip inductors and active tuned circuits, is being investigated by Plett and Khoury [Kho94] in an attempt to provide a monolithic solution to filtering for a fully integrated digital radio.

An issue that has not been dealt with in this thesis, and is mentioned here only because of its importance in radio transceiver design, is the use of wav-form coding/decoding and equalization to enhance the level of gain over channel impairments and to improve spectral efficiency. For a full radio transceiver design to be practical, some form of coding or error correction must be provided. For voice transmission, imperceptible levels of noise only reduce voice quality in a gradual manner, yet for data transmission (ie; wireless fax or modem) error rates must be minimized even at the cost of bandwidth (or increased redundancy). The use of amplitude equalization in a broadband receiver may be a requirement to allow constant envelope detection over the entire modulation bandwidth, thereby minimizing AM effects on the IF signal.

With successful work in progress on CMOS and BiCMOS frequency synthesizers, and a successfullly demonstrated implementation of a monolithic $\Delta \Sigma$ frequency discriminator, the next step in research is a printed circuit board or multi-chip module MCM radio transceiver implementation which brings the above research ideas together in an attempt to qualify a novel monolithic or hybrid broadband FM transceiver. 


\section{References}

[Bax94] W. Bax, Masters of Engineering Thesis, in progress.

[BC92] R. D. Beards, M.A. Copeland, An Oversampling Delta-Sigma Frequency Discriminator, L.E.E.E. Transactions on Circuits and Systems, Jan. 1994.

[Bea90] R.D. Beards, High Frequency BiCMOS Transconductance Integrators, M. Eng Thesis, Carleton University, 1990.

[Bea94] R. D. Beards, Doctoral Thesis, in progress.

[CB81] J.C. Candy, O.C. Benjamin, The Structure of Quantization Noise from Sigma-Delta Modulation, L.E.E.E. Transactions on Communications, Vol.Com.-29, NO. 9, September 1981, pp. 1316-1323.

[CNL90] K.C.H. Chao, S. Nadeen, W.L. Lee, C.G. Sodini, A Higher Order Topology for Interpolative Modulators for Oversampling AD Converters, I.E.E.E. Transactions on Circuits and Systems, Vol. 37, No. 3, March 1996, pp. 196-205.

[CT1] J.C. Candy, G.C. Temes, Oversampling Delta-Sigma Data Converters - Theory, Design and Simulation, IEEE Press, New York, pp. 1-29.

[Fi94] N. Filiol, Masters of Engineering Thesis, in progress.

[Har78] FJ. Harris, On the Use of Windows for Harmonic Analysis with the Discrete Fourier Transform, Proceedings of the LE.E.E., Vol. 66, No.1, January 1978, pp. 51-83.

[HP1] Hewlett-Packard, Understanding and Measuring Phase Noise in the Frequency Domain, Application note 207. 
[HP2] Hewlett-Packard Company, HP3048A Phase Noise Measurement System - Operating Manual, 1987.

[HP3] Hewlett-Packard Company, HP5372A Frequency and Time Interval Analyzer - Operating Manual, 1989.

[HP4] Hewlett-Packard Company, HP8180A Data Generator - Operating and Programming Manual, 1983.

[HP5] Hewlett-Packard Company, HP3325B Synthesizer/Function Generator-Operating Manual, 1988.

[JK90] I. Jaquet, T.A. Kwasniewski, Frequency Synthesis for Monolithic Implementation of a Gigahertz Range Transceiver. Canadian Conference on Electrical and Computer Engineering, Ottawa, Canada, 1990. pp. 4.3.1-4.3.3.

[KBR80] H.L. Krauss, CW. Bostian, F.H. Raab, Solid State Radio Engineering. John Wiley \& Sensinc, New York, 1980.

[KCOM] KELCOM, A Division of K\&L Microwave, Inc., Salisbury, Maryland.

[Kho94] G. Khoury, Masters of Engineering Thesis, in progress.

[Lon94] J.R. Long, Doctoral Thesis, in progress.

[Lon88] L.L. Longo, Multi-Stages Sigma Delta Modulators, Masters Thesis, Carleton University, Ottawa, April 1988.

[Mat92] The Math Works Inc., MATLAB Reference Guide, Natick Mass., 1992.

[MCI] Mini-Cincuits, RF/IF Designer's Handbook, Brooklyn, New York. 1993, pp. 1-8, 1-38.

[MC90] B. Miller, B. Conley, A Multiple Modulator Fractional Divider, Proceedings of the 44th Annual Symposium on Frequency Control, May 1990, pp. 559-567.

[Mot1] Motorola Inc., MECL Device Dasa, fourth edition, 1989. 
[Mot2] Motorola Inc., Motorola MECL System Design Handbwok, 1st edition, 1988.

[Mot3] Motorola Inc., ECL in PS Data Book, rev 1, 1991.

[NS93] National Semiconductor Corporation, LMX2216B 0.1CHz to $2.0 \mathrm{GHz}$ Low Noise Amplifier/Mixer for RF Personal Communications, Preliminary Data Sheet, 1993.

[Nut81] A.H. Nuttal, Sume Windows with Very Good Sidelobe Behaviour, I.E.E.E. Transactions on Acoustics, Speech, and Signal Frocessing, Vol. ASSP-29, No.1,February 1981, pp. 84.-91.

[Oga70] K. Ogata, Modern Control Engineering, Prentice-Hall, Inc., Englewood Cliffs, N.J., 1970, p. 70.

[Ples88] Plessey Semiconductors, Frequency Dividers and Synthesizers IC Handbook. The Plessey Company plc, 1988.

[PL92] S.C. Pinault, P.V. Lopresti, On the Behaviour of the Double Loop Sigma Delsa Modulator-II: Sinuso: al Inputs, AT\&T Bell Labratories, Engineering Research Center, 1992.

[PM91] D.O. Pederson, K. Maryaram, Analog Integrated Circuits for Communication, Principles, Simulation and Design, Kluwer Academic Publishing, Boston, 1991.

[P090] Ian Poole, Synthesizers Noise, Electronics World \& Wireless World, September 1990, pp. 820-825.

[Pro89] J.C. Proakis, Digital Communications, 2nd edition, McGraw-Hill Book Company, New York, 1989.

[RC92a] T.A. Riley, M.A. Copeland, Use of the Phase Portrait to define bounds and stability of Delta Sigma Modulator, an internal document, Carleton University, Dept. of Electronics, April 1992.

[RC92b] T.A. Riley, M.A. Copeland, A Comparison Between a lst order $\Delta \mathbf{\Sigma}$ Modulator and a lst Order DPA, An intermal document, Carleton university, 1992. 
[RC92c] T.A. Riley, M.A. Copelands, The Effects of Cyclostationary Phase Noise on Randomized Noise $\Delta-\Sigma$ Modulator Synthesizers, an internal document, Carleton University, Dept. of Electronics, 1992.

[RCK93] T.A. Riley, M.A. Copeland, T.A. Kwasniewski, Delta-Sigma Modulation in Fractional-N Frequency Synthesis, IEEE Joumal of SolidState Circuits, Vol 28, No. 5, May 1993, pp. 553-559.

[Rob91] W.P. Robins, Phase Noise in Signat Sources, Peter Peregrinus Ltd., London, United Kingdom, 1991.

[Ril] T.A. Riley, Personal Communications, Carleton University, Dept. of Electronics.

[Ri2] T.A. Riley, Oversampled analog to digital conversion evaluation software - ROSAD, 2nd ed., Carleton University, Dept. of Electronics. 1991.

[Ri3] T.A. Riley, M.A. Copeland. TRIO, U.S. Patent 4965531

[Sor91] W. Sorfleet, Noise and Transient Analysis of a Fraction-N Phase Locked Loop Frequency Synthesizer, Masters Thesis, Carleton University, Dept. of Electronics. April 1991.

[Spi] L.G. Meares, C.E. Hymowitz. Simulating with Spice, Intusoft, 1988.

[SR92] Siemens Review R\&D Special; Siemens Aktiengesellschaft, 1992, pg 16-19.

[SS89] Sony Inc, SPECL Standard Logic Family Semiconductor Integrated Circuit Data Book. 1989.

[Str79] F.G. Stremler, Introduction to Communication Systems, AddisonWesley Publishing Company, Reading, Massachusetts, 1979, pp. 269277.

[Th93] M. Thamsirianunt, Masters of Enginsering Thesis, in progress.

[UR83] U.L. Rohde, Digital PLL Frequency Synthesizers, Prentice-Hall Inc. Englewond Cliff, N.J., 1983. 
[Wat86] R. Watson, Guidelines for Receiver Analysis, Microwaves \& Rf, December, 1986, pp. 113-122.

[WW91] L.A. Williams, B.A. Wooley, Third-Order Cascaded Sigma Delta Modulators I.E.E.E. Transactions on Circuits and Systems, Vol. 38. No. 5, May 1991,pp. 489-498.

[Xi192] Xilinx Inc., The Programmable Gate Array Data Book, 1992.

[Zcom] Z-Communication Inc., VCO Product Catalog, 1992.

[Zho91] M. Zhong, A 3 $\mu M \Sigma-\Delta$ Modulator and Dual Modulus Divider for use in $\Sigma-\Delta$ Modulated Fractional-N Frequency Synthesis, Masters thesis, Carleton University, Dept. of Electronics, 1991.

[ZT85] R.E. Ziemer, W.H. Tranter, Principles of CommunicationSystems, Modulation, and Noise, 2nd ed., Houghton Mifflin Company, Boston, 1985. 


\section{Appendix A}

\section{PLL Circuits}

This section contains a three page wiring diagram and two page parts list for the PLL presented in Chapter 3. 


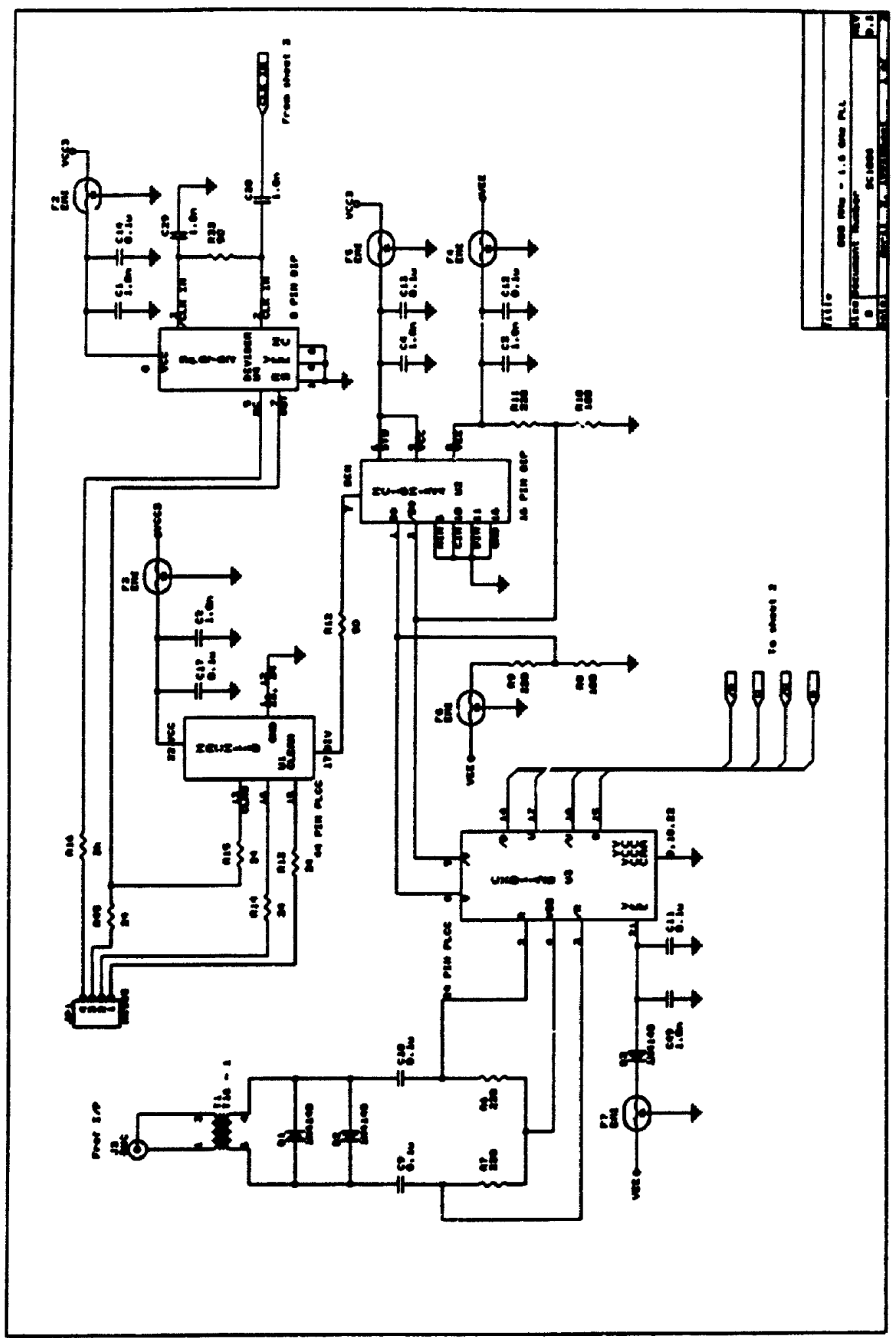




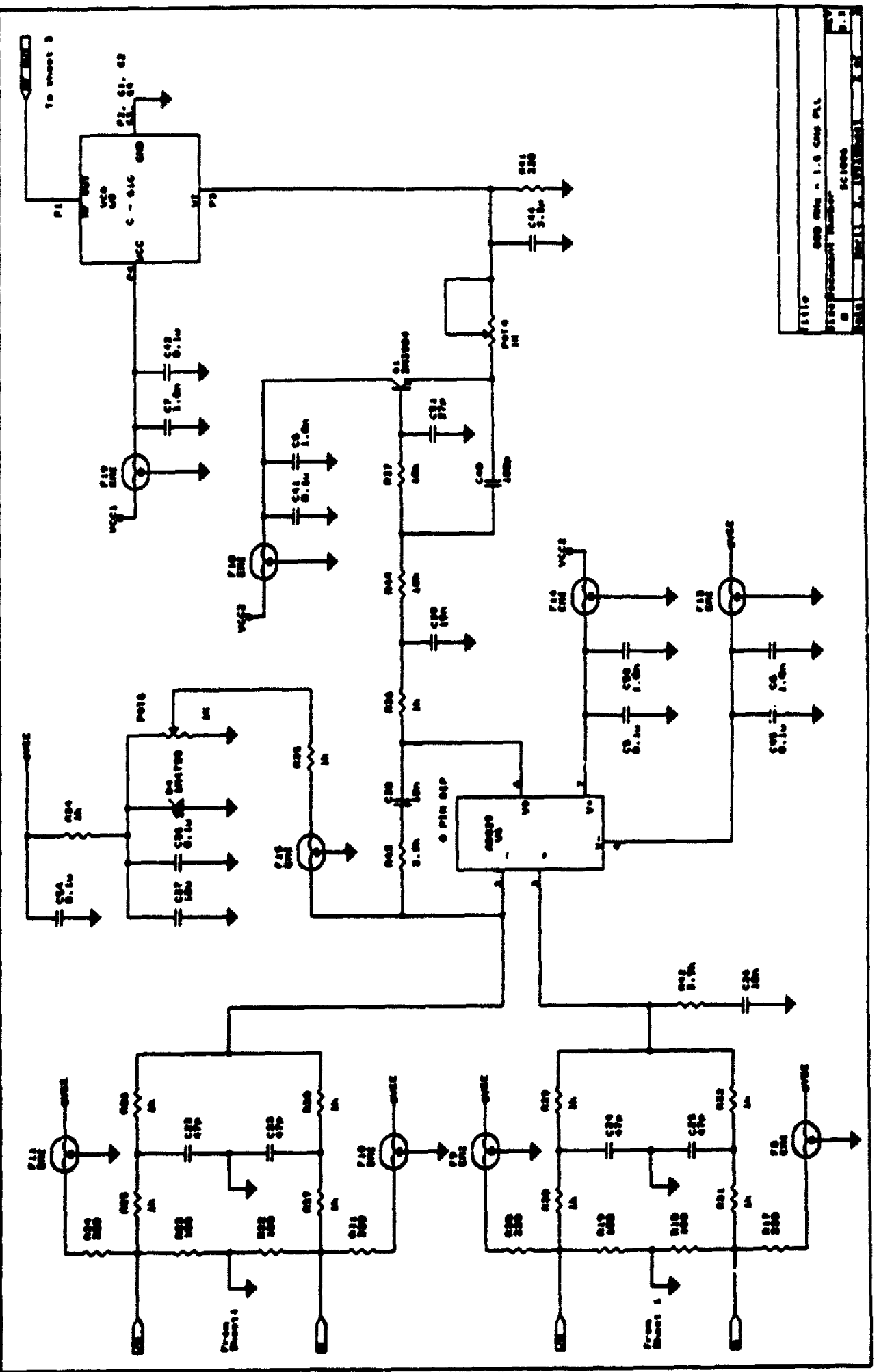




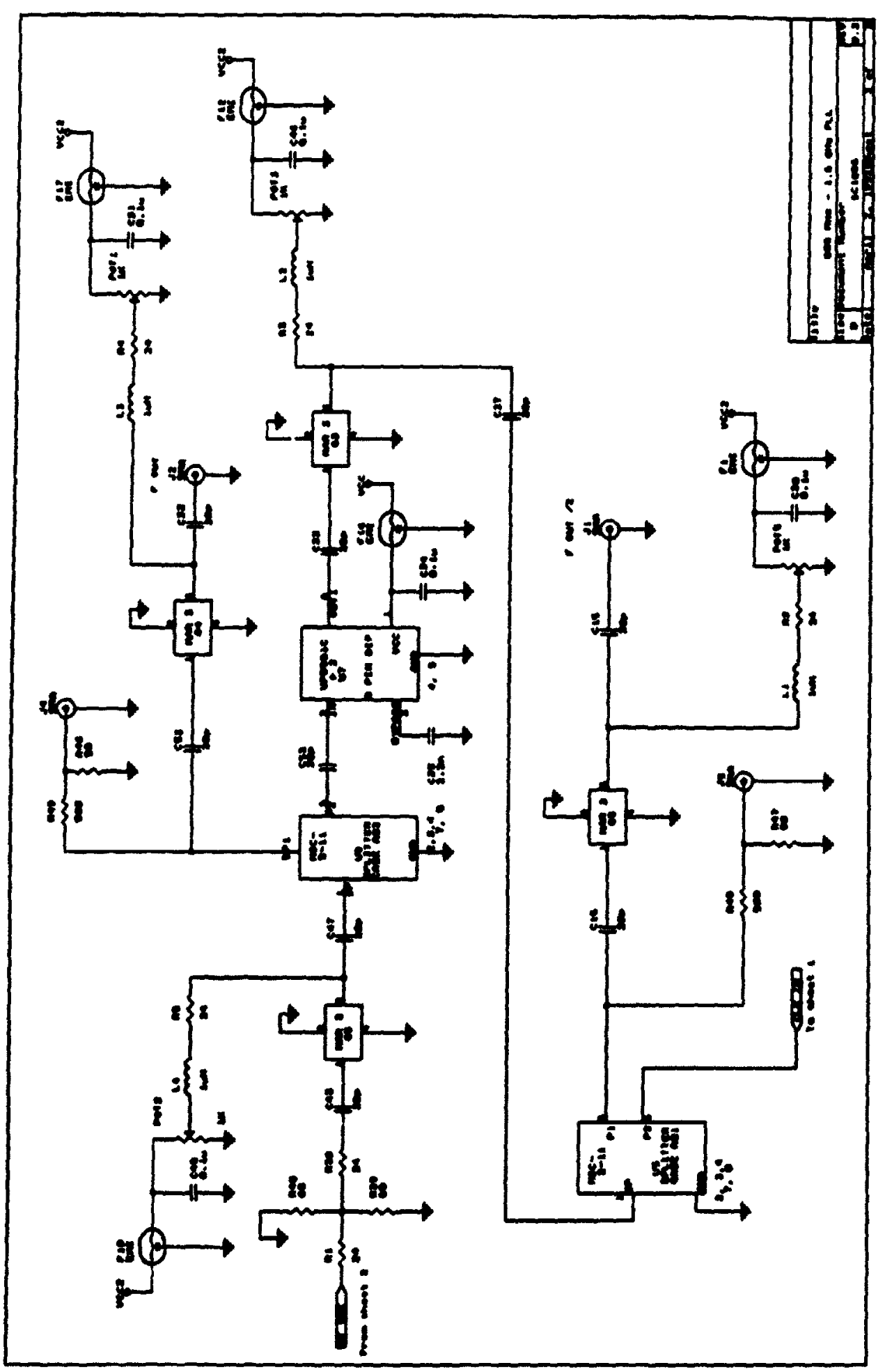


$800 \mathrm{MHz}-1.6 \mathrm{GHz}$ PLL Parts List January 12, 1999

\begin{tabular}{|c|c|c|c|}
\hline Item & Quantity & Reference & Part \\
\hline 1 & 11 & $\begin{array}{l}\mathrm{C} 1, \mathrm{C} 2, \mathrm{C} 3, \mathrm{C} 4, \mathrm{C} 6, \mathrm{C} 7, \mathrm{C} 8, \mathrm{C} 28, \\
\mathrm{C} 29, \mathrm{C} 49, \mathrm{C} 50\end{array}$ & $1.0 \mathrm{nf}$ \\
\hline 2 & 18 & 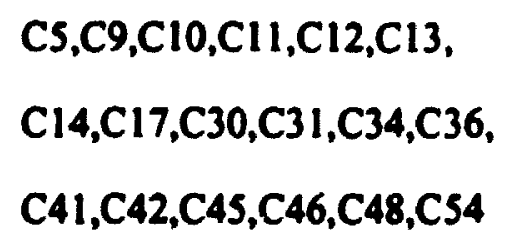 & $0.1 \mu \mathrm{f}$ \\
\hline 3 & 9 & $\begin{array}{l}\mathrm{C} 15, \mathrm{C} 16, \mathrm{C} 27, \mathrm{C} 32, \mathrm{C} 33, \mathrm{C} 43, \\
\mathrm{C} 47, \mathrm{C} 52, \mathrm{C} 53\end{array}$ & $20 \mathrm{pf}$ \\
\hline 4 & 8 & $\begin{array}{l}\mathrm{C} 18, \mathrm{C} 19, \mathrm{C} 20, \mathrm{C} 21, \mathrm{C} 22, \mathrm{C} 23, \\
\mathrm{C} 24, \mathrm{C} 25\end{array}$ & $47 \mathrm{pf}$ \\
\hline 5 & 2 & C26,C38 & $18 \mathrm{nf}$ \\
\hline 6 & 1 & C35 & $2.2 \mathrm{nf}$ \\
\hline 7 & 1 & C37 & $10 \mu \mathrm{f}$ \\
\hline 8 & 1 & C39 & $15 \mathrm{nf}$ \\
\hline 9 & 1 & $\mathrm{C} 4 \mathrm{O}$ & $100 \mathrm{pf}$ \\
\hline 10 & 1 & C44 & 3.3pf \\
\hline 11 & 1 & Cs1 & $27 \mathrm{pf}$ \\
\hline 12 & 3 & $\mathrm{D} 1, \mathrm{D} 2, \mathrm{D} 3$ & IN4148 \\
\hline 13 & 1 & D4 & IN4728 \\
\hline
\end{tabular}


151

JPI

$\mathrm{J1}, \mathrm{J} 2, \mathrm{J4}, \mathrm{J5}$

J3

171

184

196

$20 \quad 11$

11

L1,L2,L3,LA

POT 1,POT2,POT3,POT4,POTS,

POT6

R25,R26,R27,R28,R29,R30

R31,R32,R34,R35,R36

$21 \quad 1$

$22 \quad 10$

Q1

$\mathbf{R 1 , R 2 , R 3 , R 4 , R S , R 1 3 , R 1 4 , ~}$

R15,R38,R45

239

R6,R7,R9,R11,R17,R20,R21, R24,R41

246

254

261

$27 \quad 2$

$28 \quad 2$

$29 \quad 2$

302

311

$32 \quad 2$

331
R8,R10,R18,R19,R22,R23

R12,R33,R46,R49

R16

R37,R44

R39,R40

R42,R43

R48,R47

T1

UI

U2
H2565

SMA

BNC

Iuh

$1 \mathrm{k} \Omega$

$1 \mathrm{k} \Omega$

2N3904

$24 \Omega$

$220 \Omega$

$100 \Omega$

$50 \Omega$

$3 \mathbf{k} \Omega$

$10 \mathrm{k} \Omega$

$68 \Omega$

$3.9 \mathrm{k} \Omega$

$500 \Omega$

T16 - 1

MACHIIO

MC1OH124 


$\begin{array}{lll}34 & 1 & \mathrm{U} 3 \\ 35 & 1 & \mathrm{U} 4 \\ 36 & 4 & \mathrm{Q} 2, \mathrm{Q}, \mathrm{Q}, \mathrm{Q}, \mathrm{QS} \\ 37 & 1 & \mathrm{U} 6 \\ 38 & 1 & \mathrm{U} 7 \\ 39 & 2 & \mathrm{U} 5, \mathrm{U} 8 \\ 40 & 1 & \text { U9 }\end{array}$

CXB112Q

SP8782

MAR 3

AD829

UPB581C

MSC-2-11

C-616 (VCO) 


\section{Appendix B}

\section{The Third Order Digital $\Delta \Sigma$ Modulator Circuit}

This section contains a wiring diagram and parts list for the printed circuit board design of the digital $\Delta \Sigma$ modulator. It also contains the full set of schematic diagrams for the third order digital $\Delta \Sigma$ modulator design, implemented in a single field programmable gate array (LCA). 


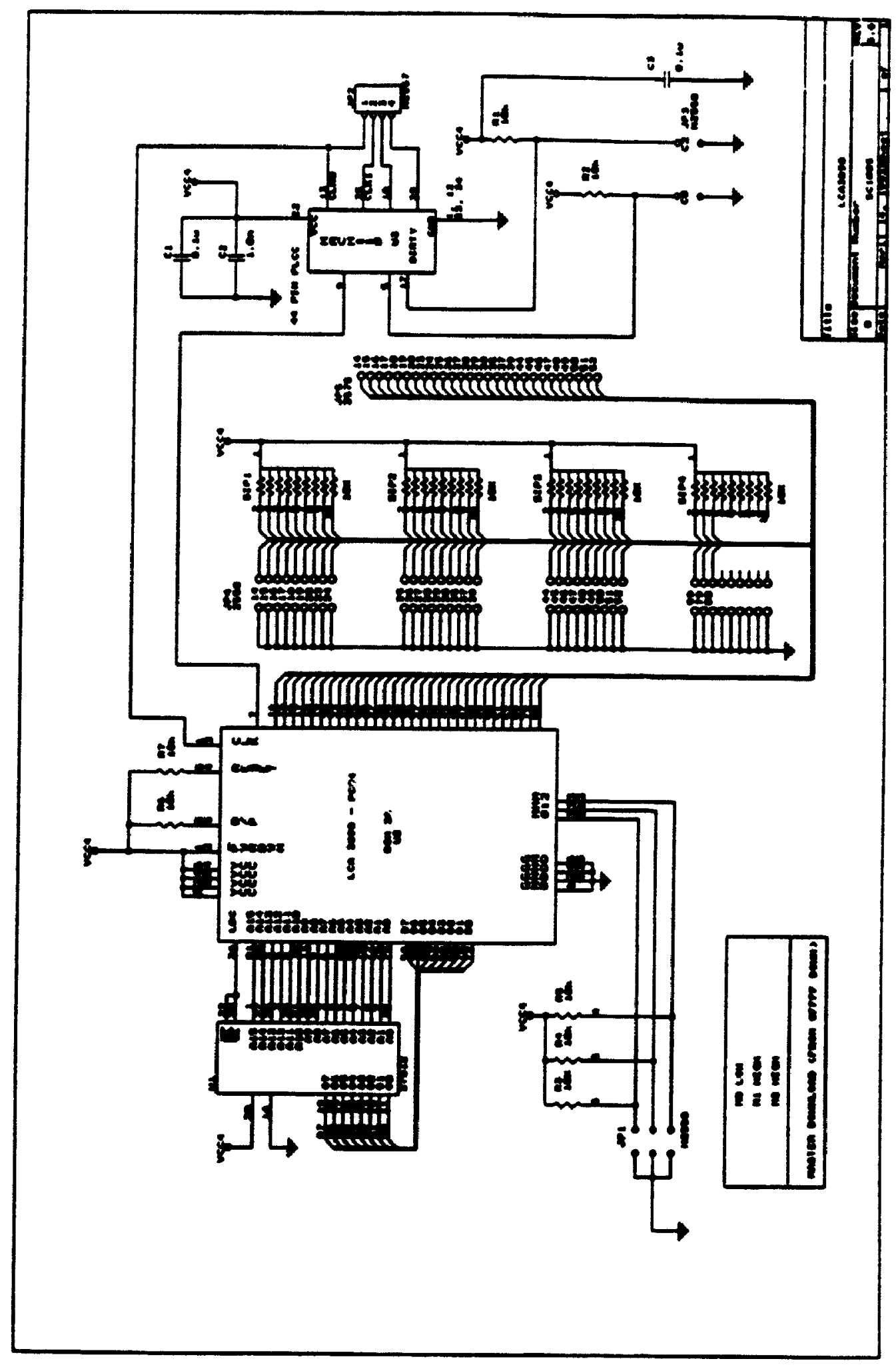




\begin{tabular}{|c|c|c|c|}
\hline LCA & Board & Janury 3, 1999 & \\
\hline Item & Quantity & Reference & Part \\
\hline 1 & 2 & $\mathrm{Cl}, \mathrm{C3}$ & $0.1 \mu f$ \\
\hline 2 & 1 & C2 & $1.0 \mathrm{nf}$ \\
\hline 3 & 3 & JP1,JP3,JP4 & H2558 \\
\hline 4 & 1 & JP2 & H2557 \\
\hline 5 & 1 & JPS & H3575 \\
\hline 6 & 7 & $R 1, R 2, R 3, R 4, R S, R 6, R 7$ & $10 \mathrm{k} \Omega$ \\
\hline 7 & 4 & SIP1,SIP2,SIP3,SIP4 & $10 \mathrm{k} \Omega$ \\
\hline 8 & 1 & Uı & 27512 (Eprom) \\
\hline 9 & 1 & U2 & LCA3090 (FPGA) \\
\hline 1C & 1 & U3 & MACH110 (PLD) \\
\hline
\end{tabular}




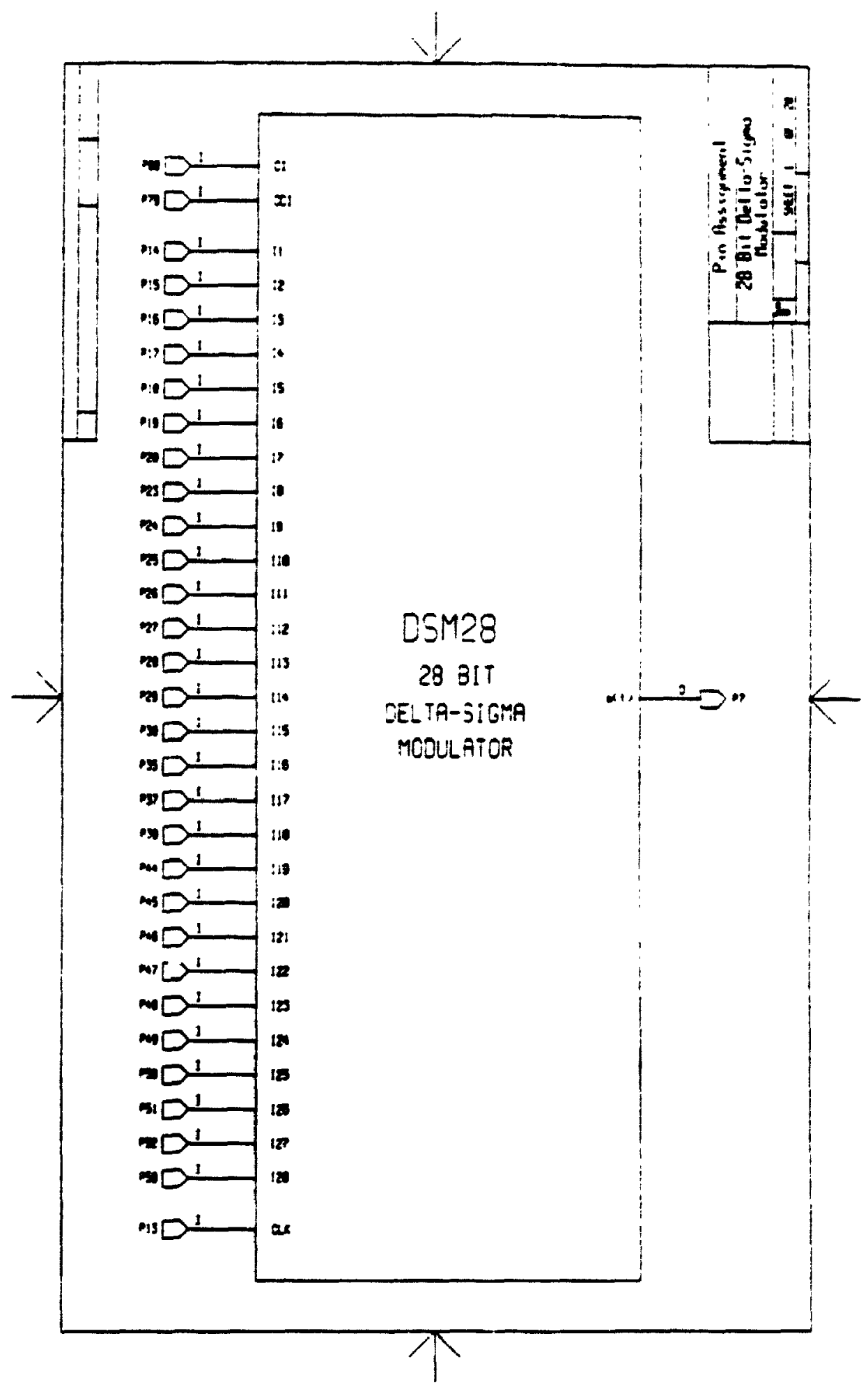




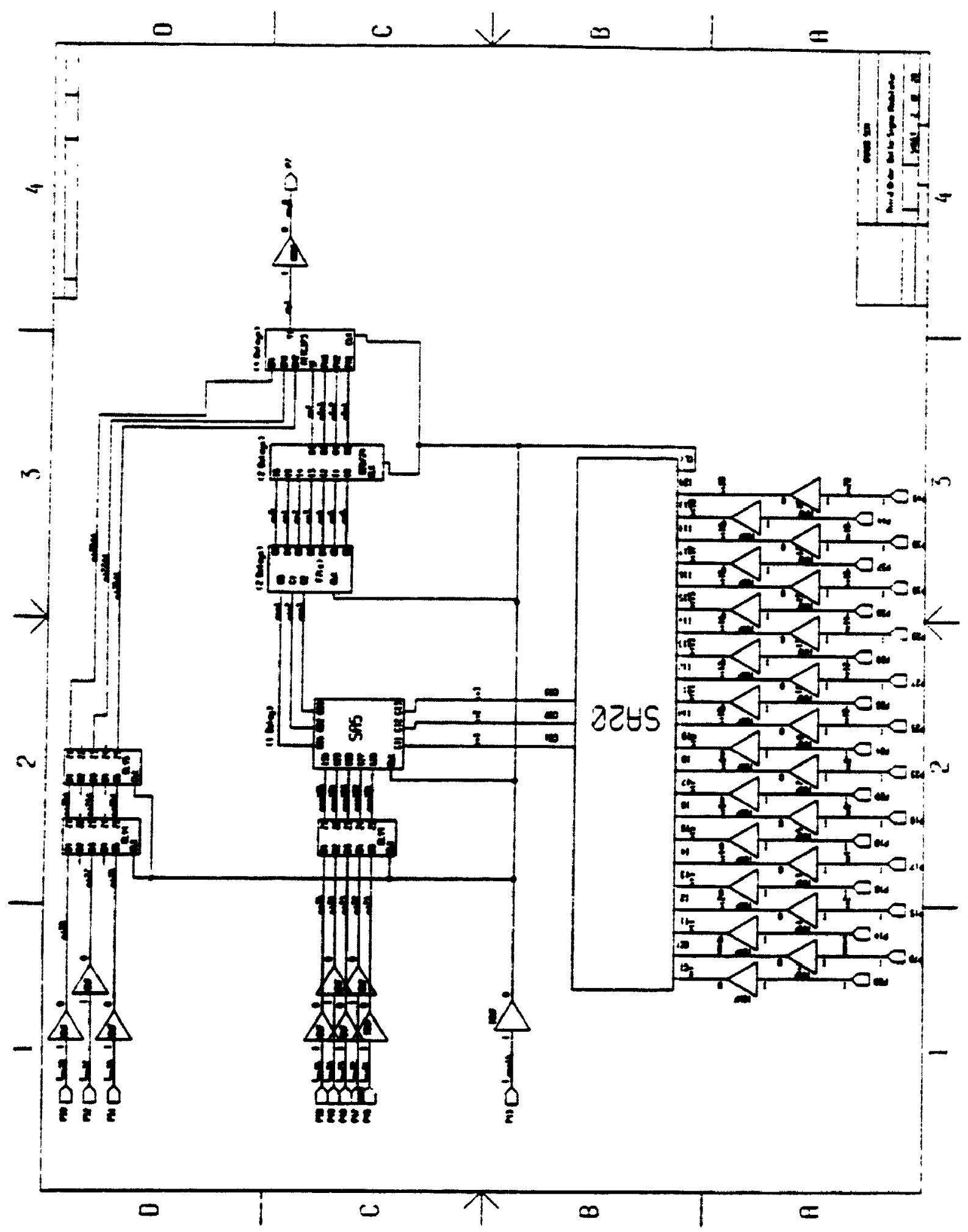




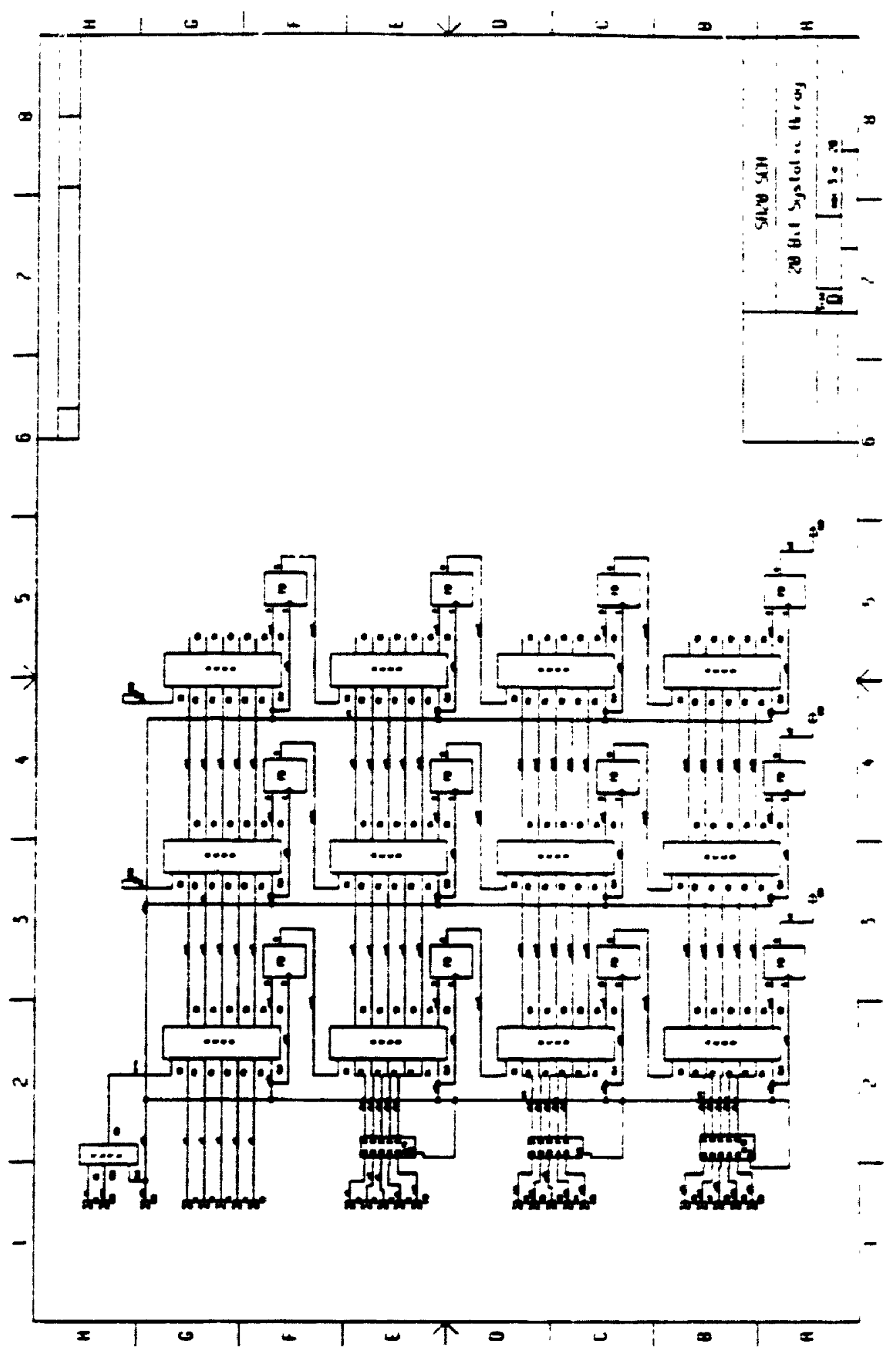




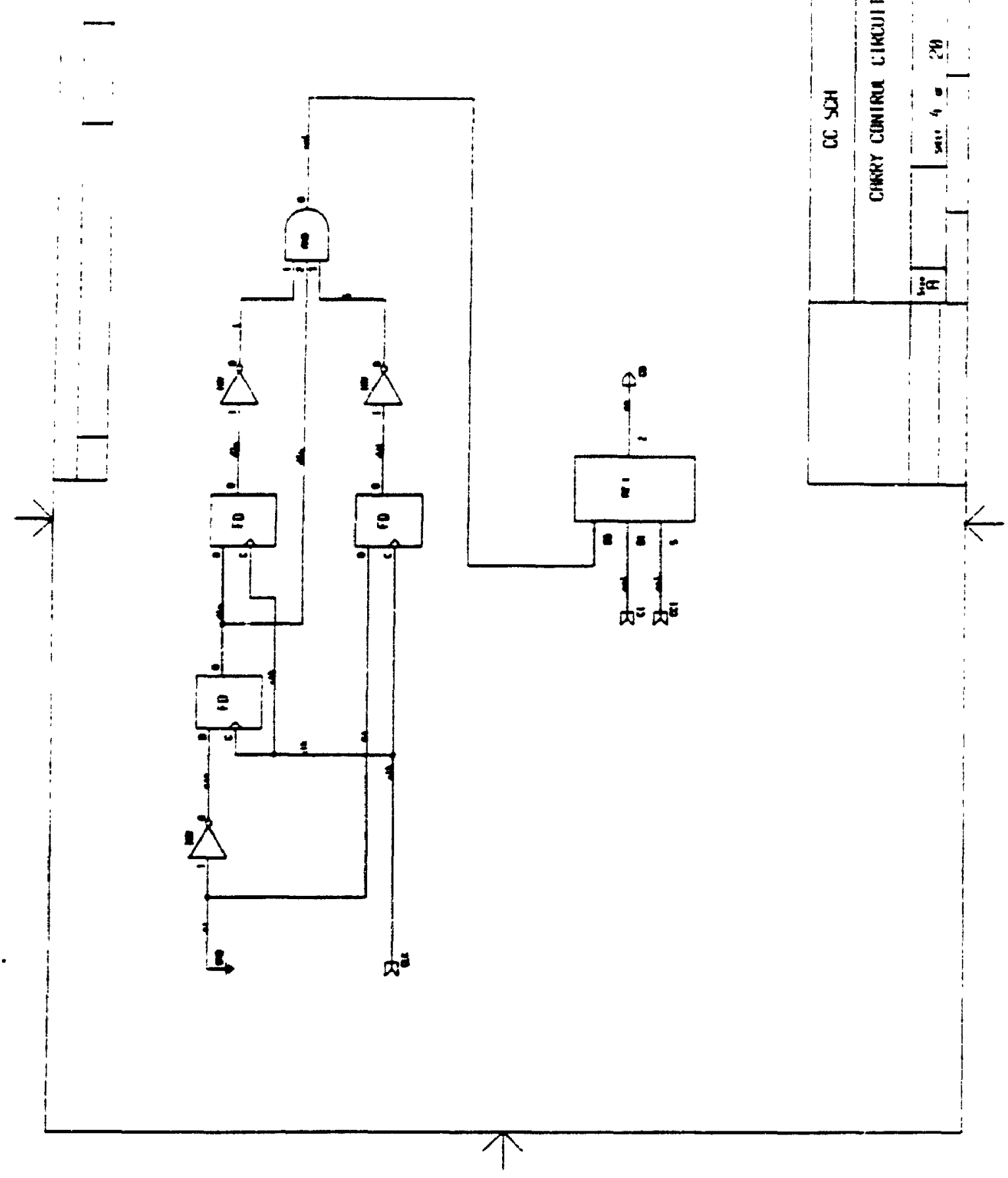




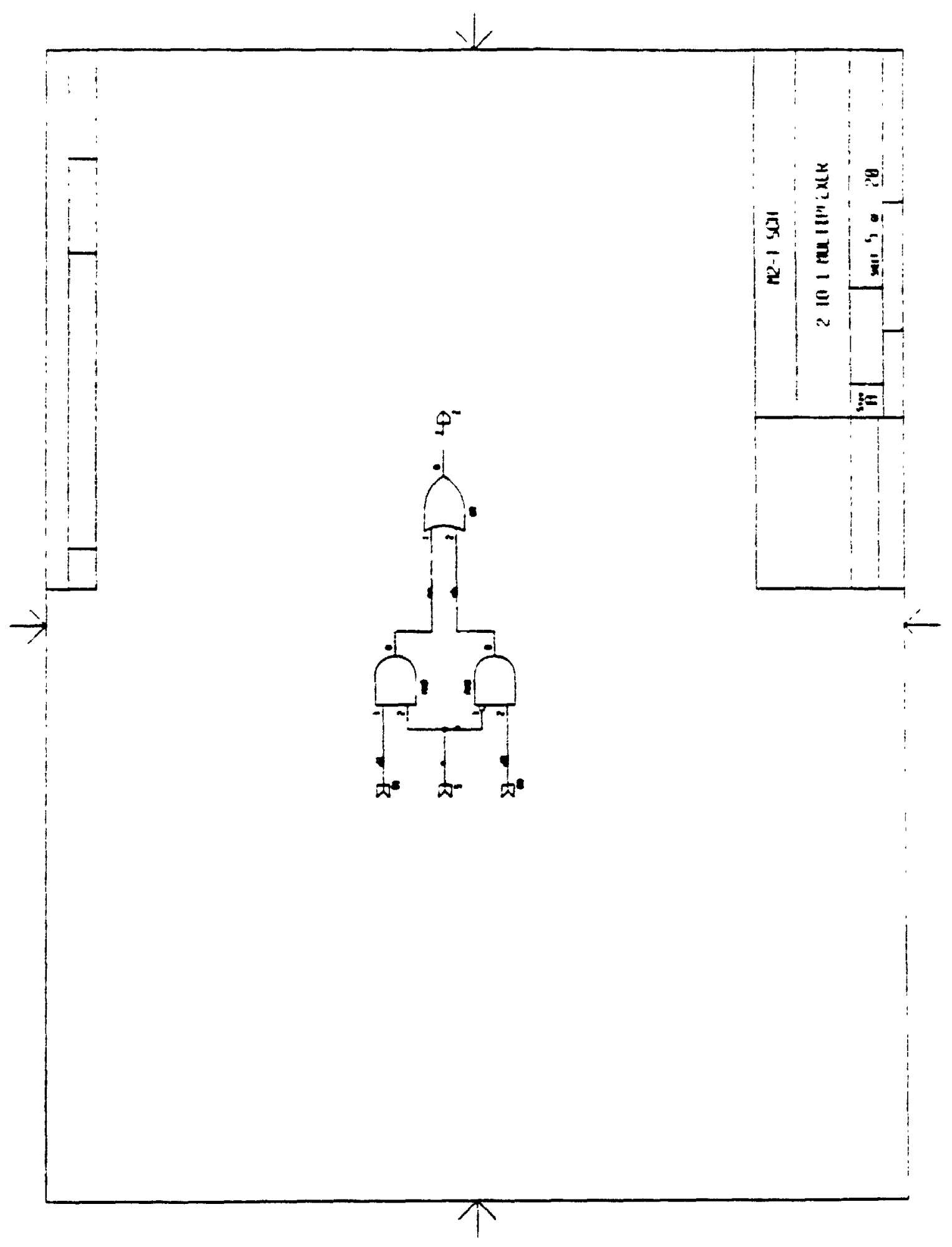

B.8 


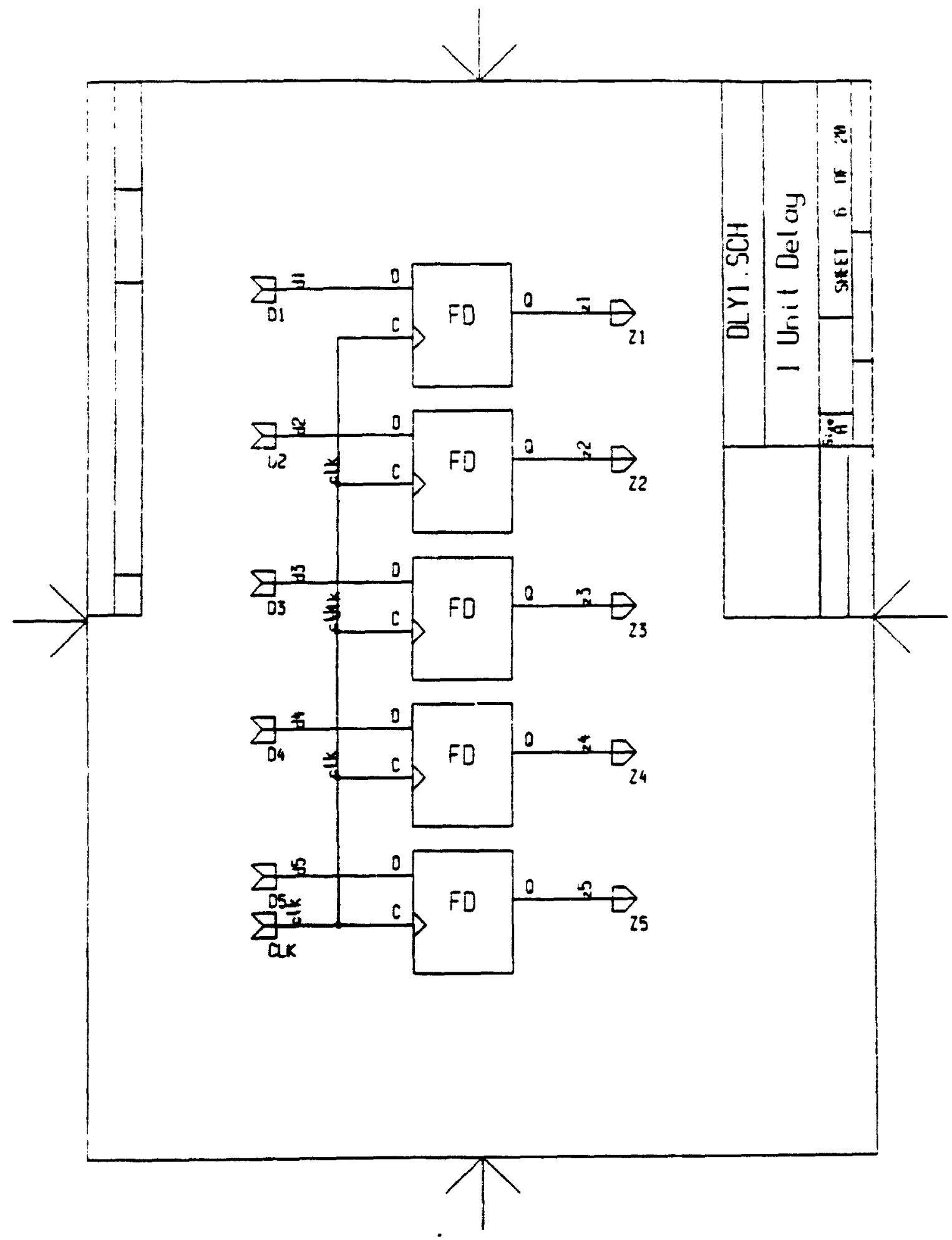

B.9 


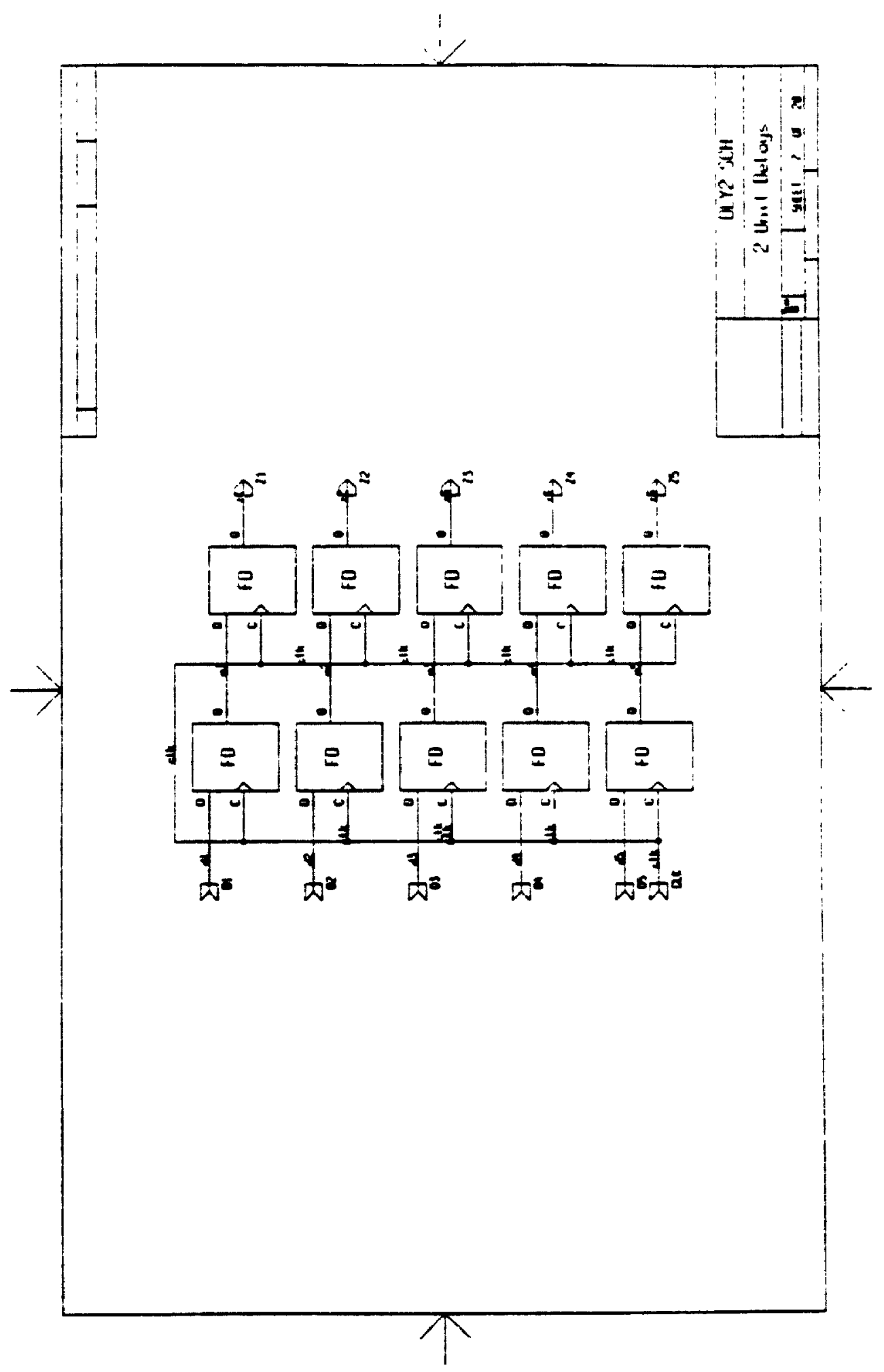

B. 10 


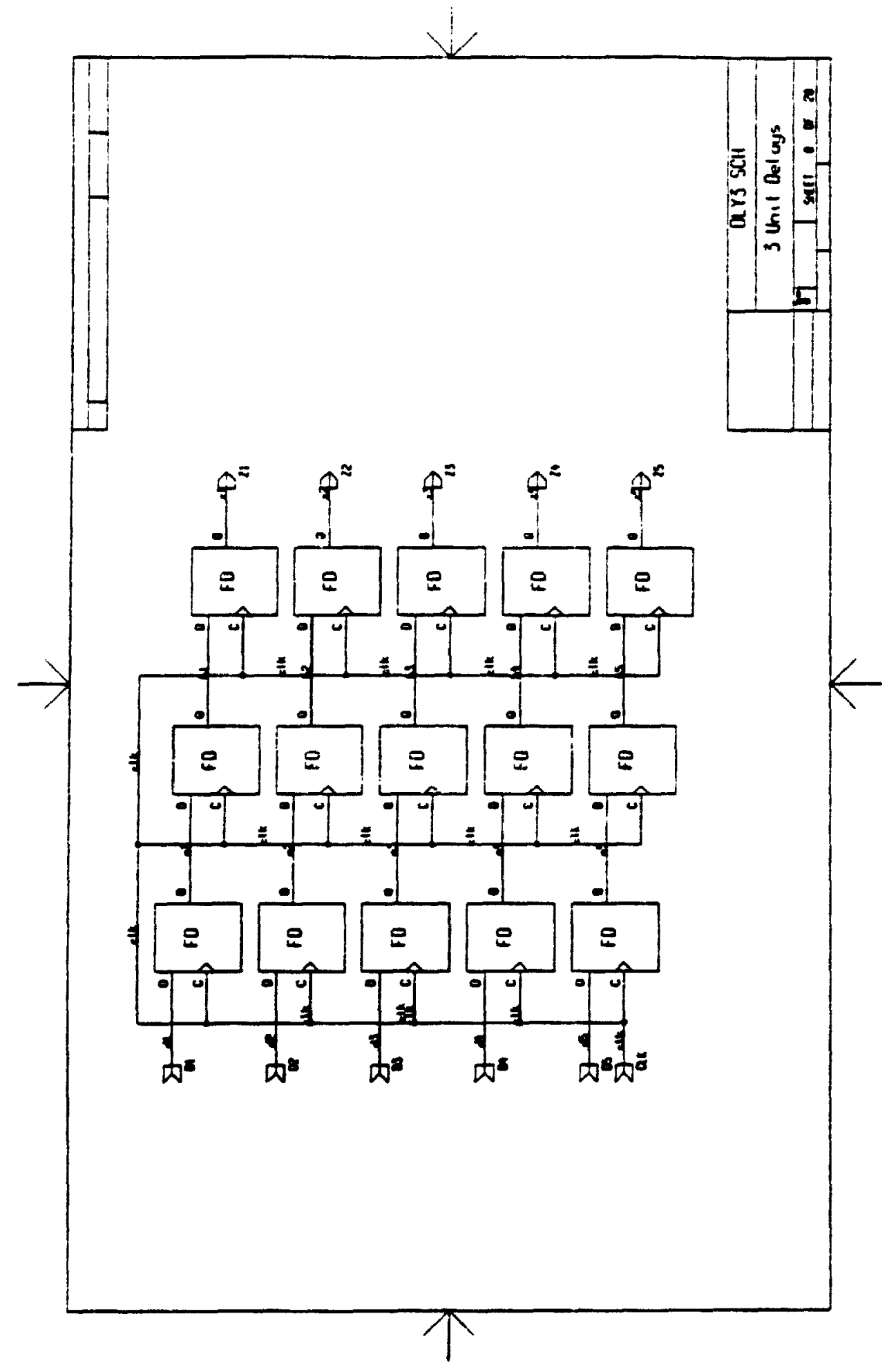

B.11 


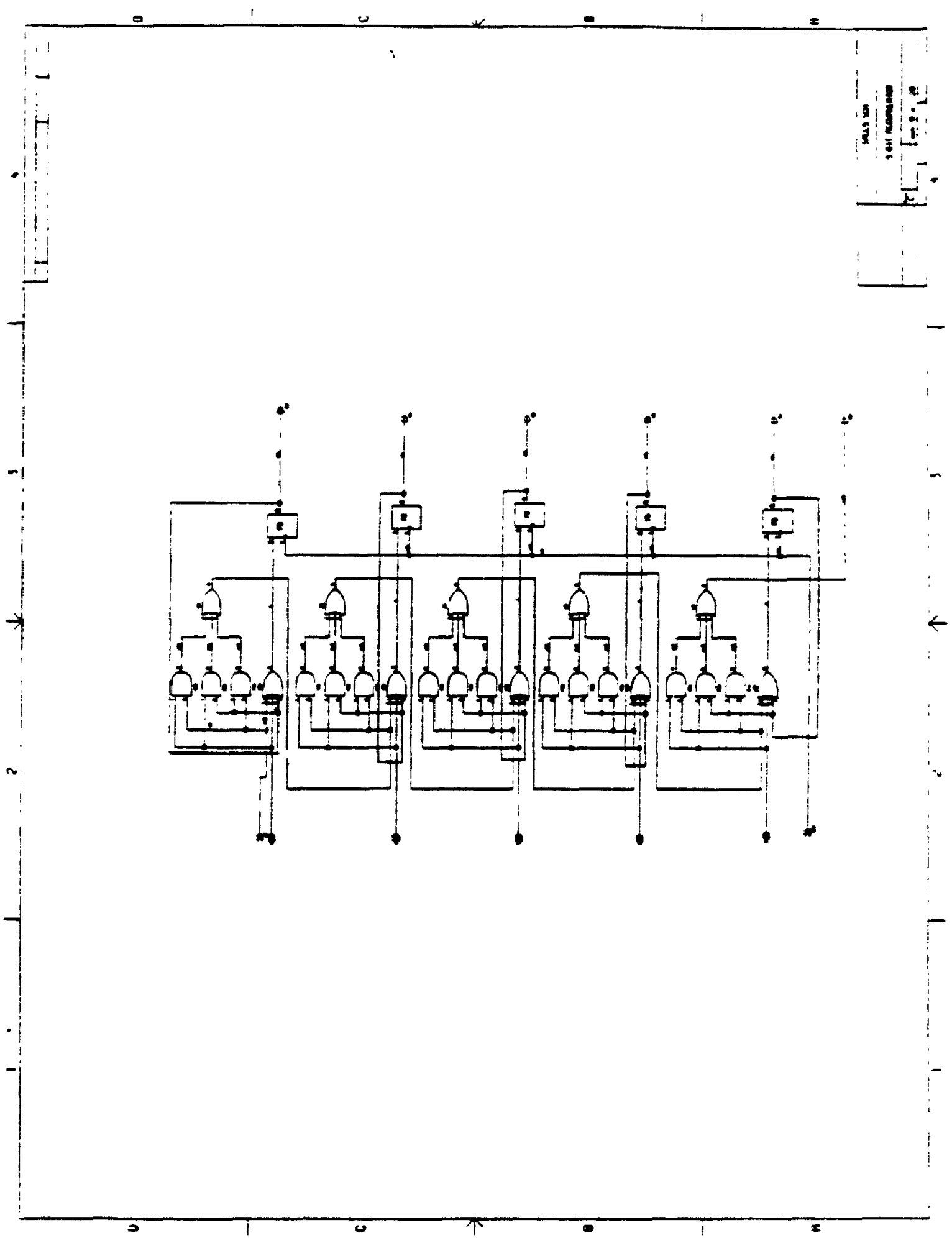




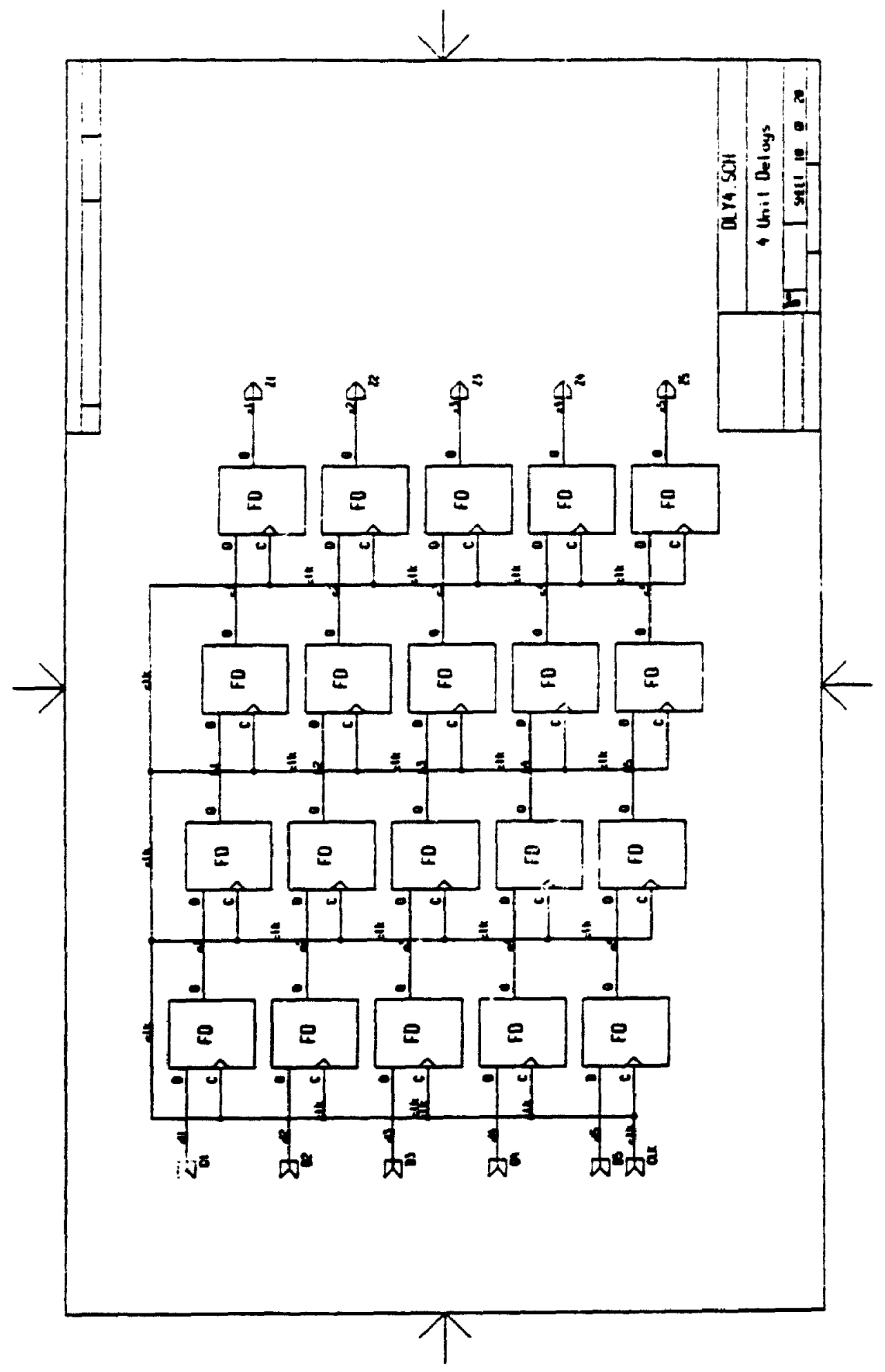




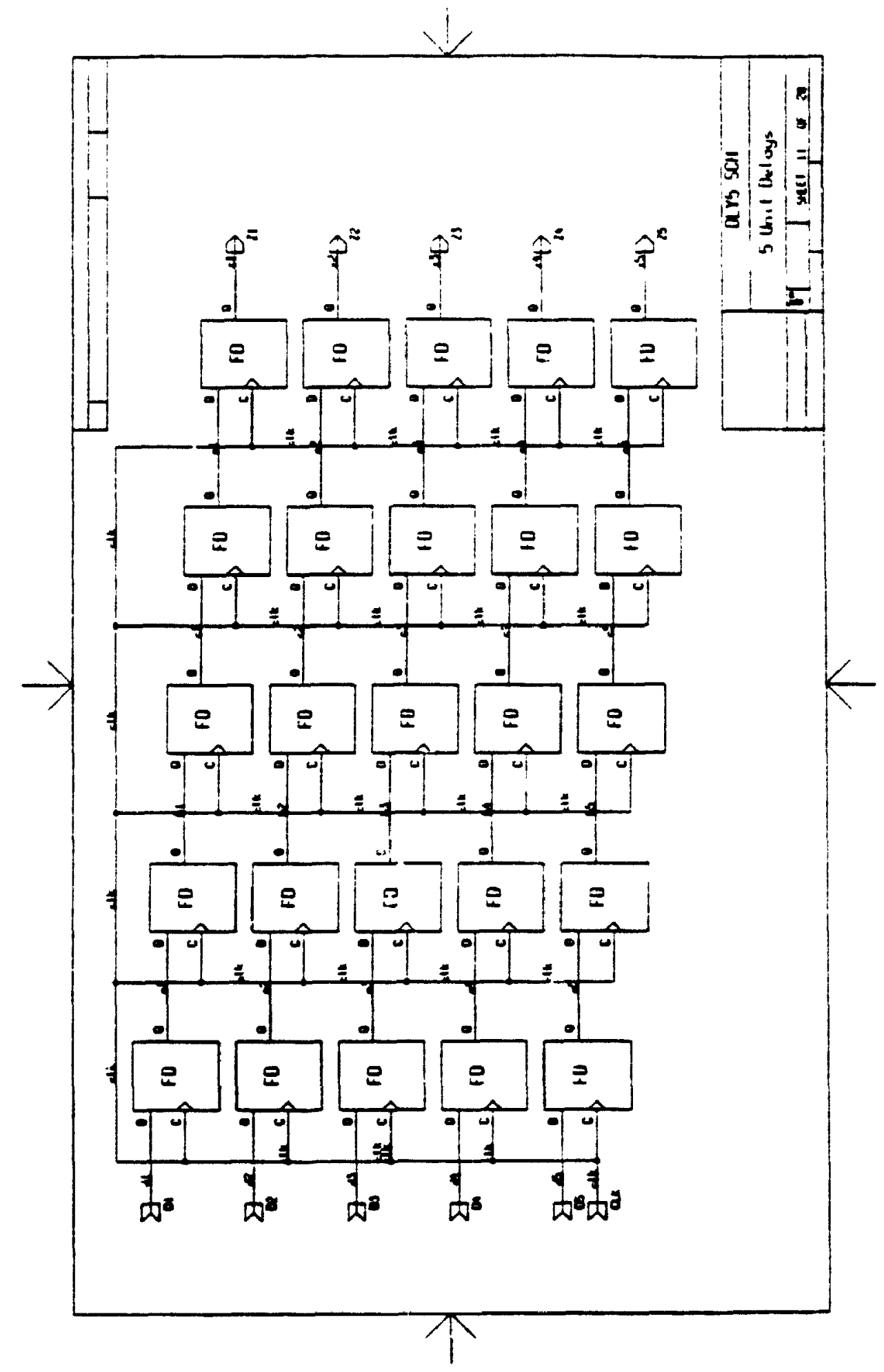

B.14 


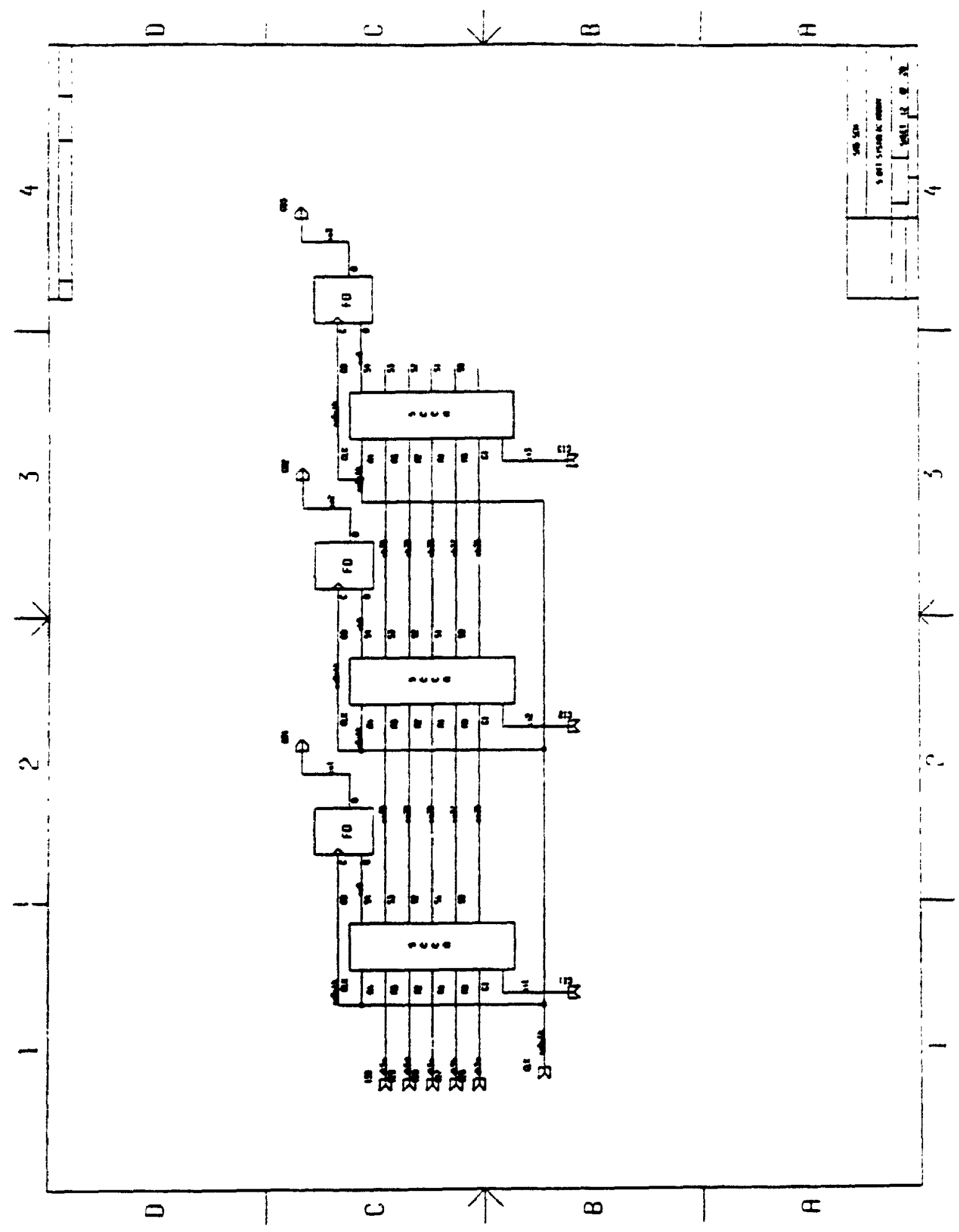




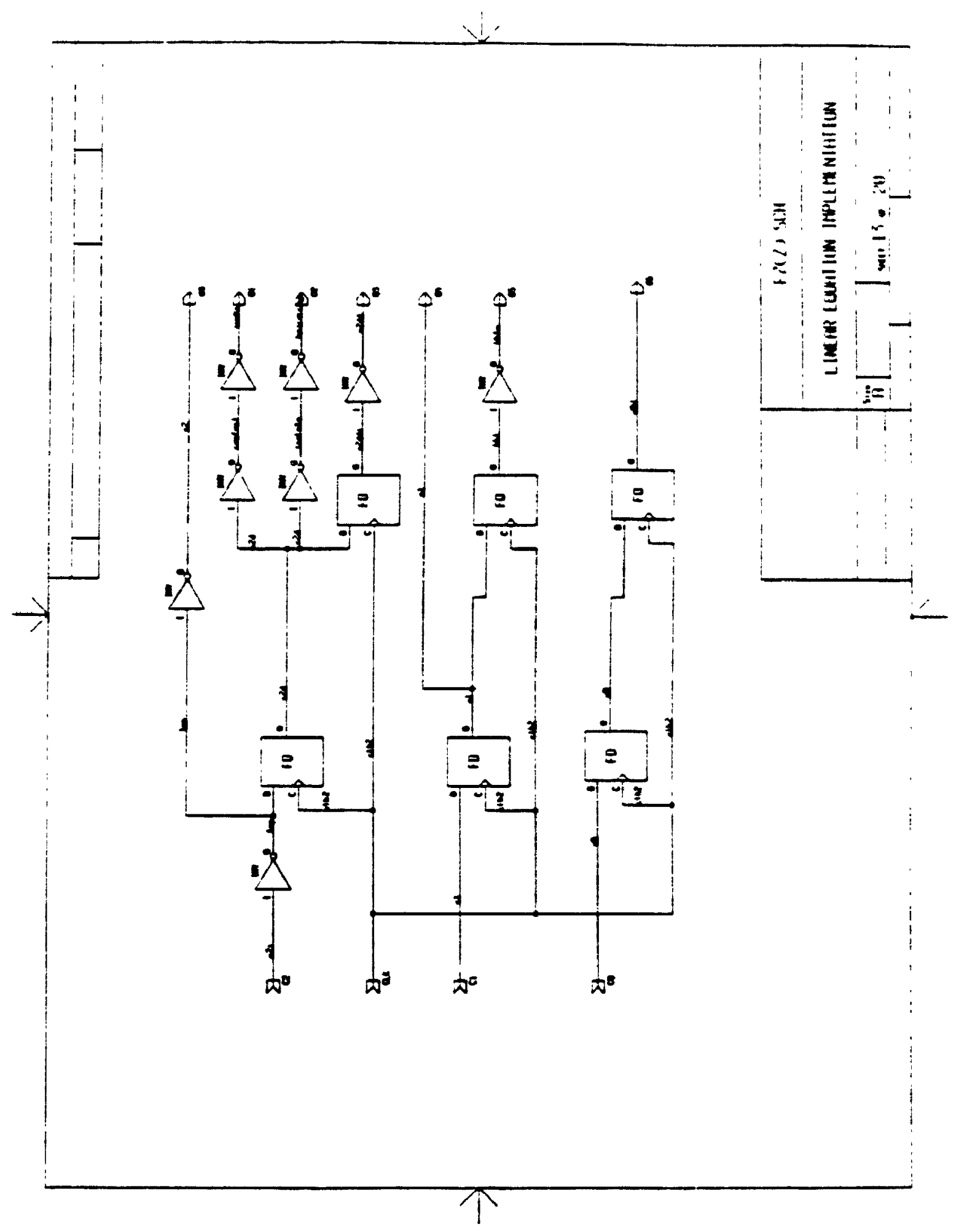




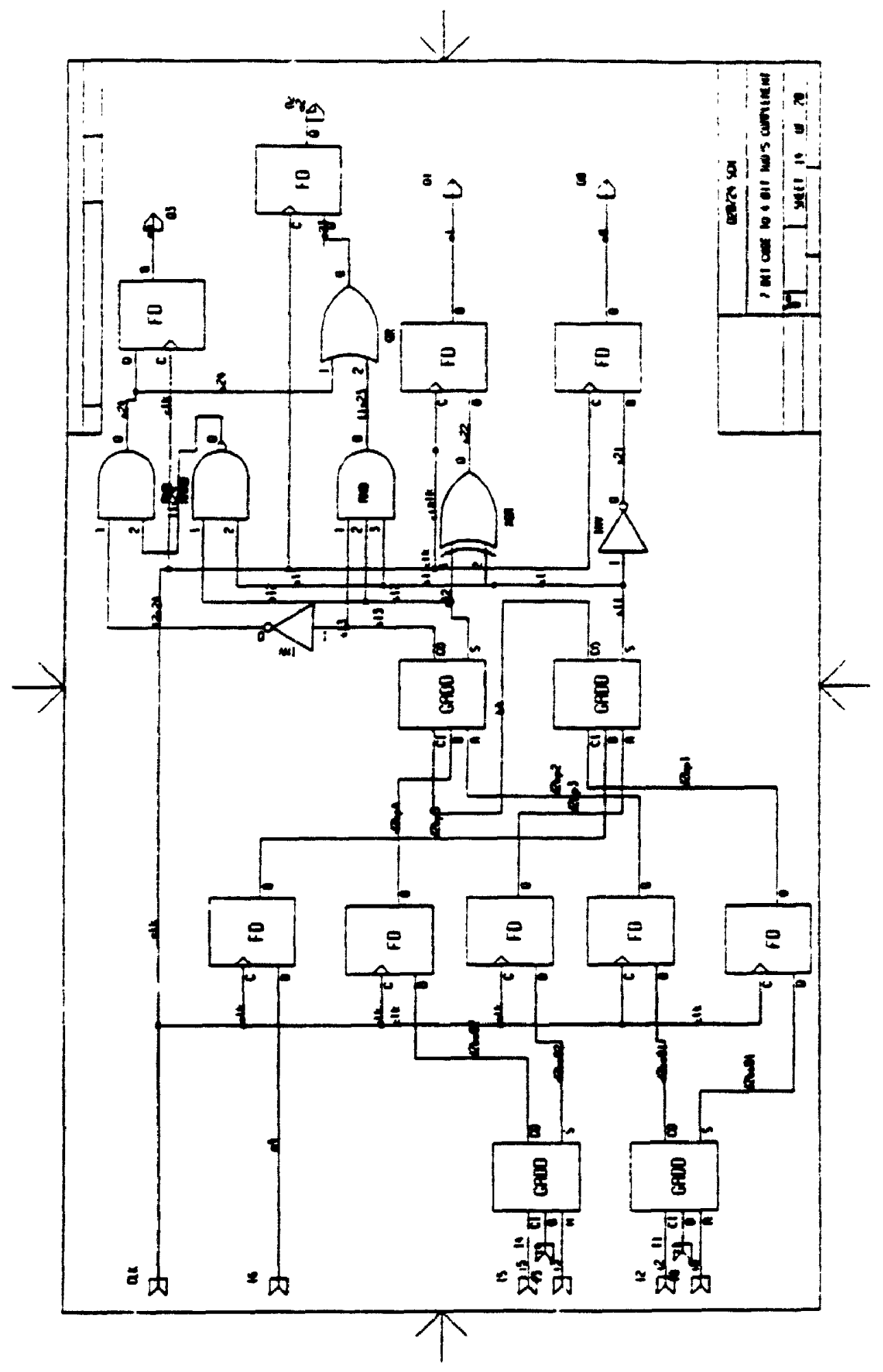




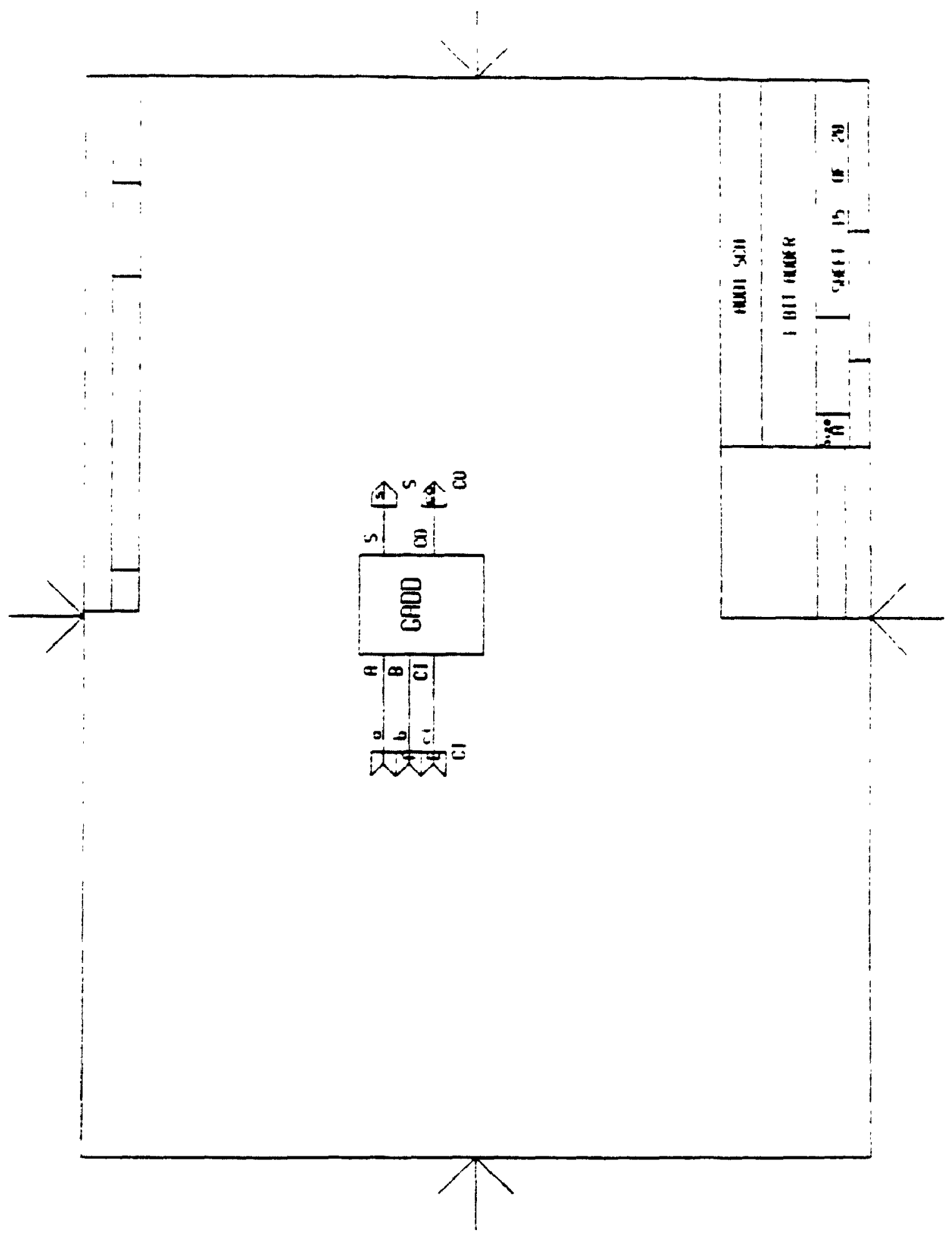

B.18 


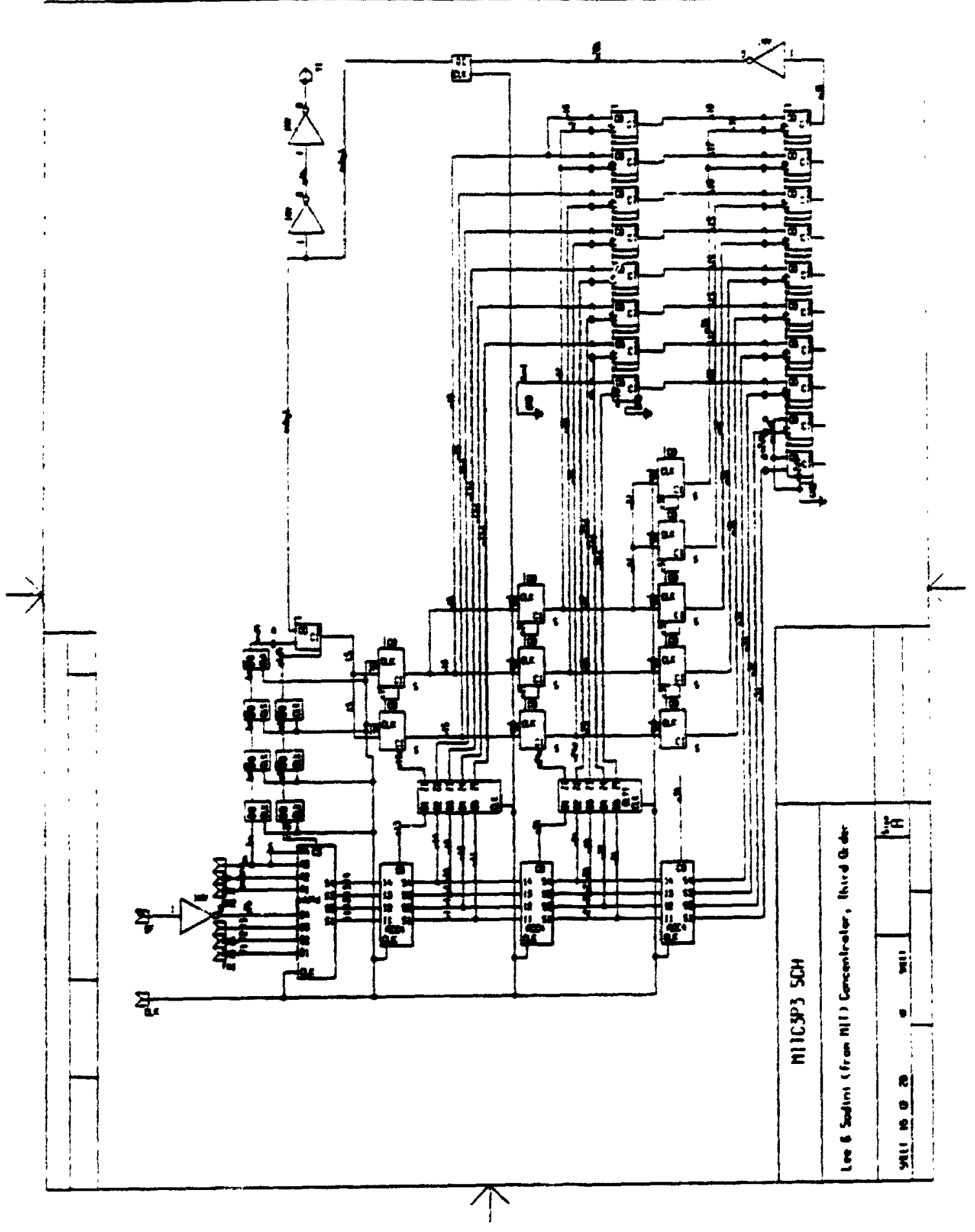




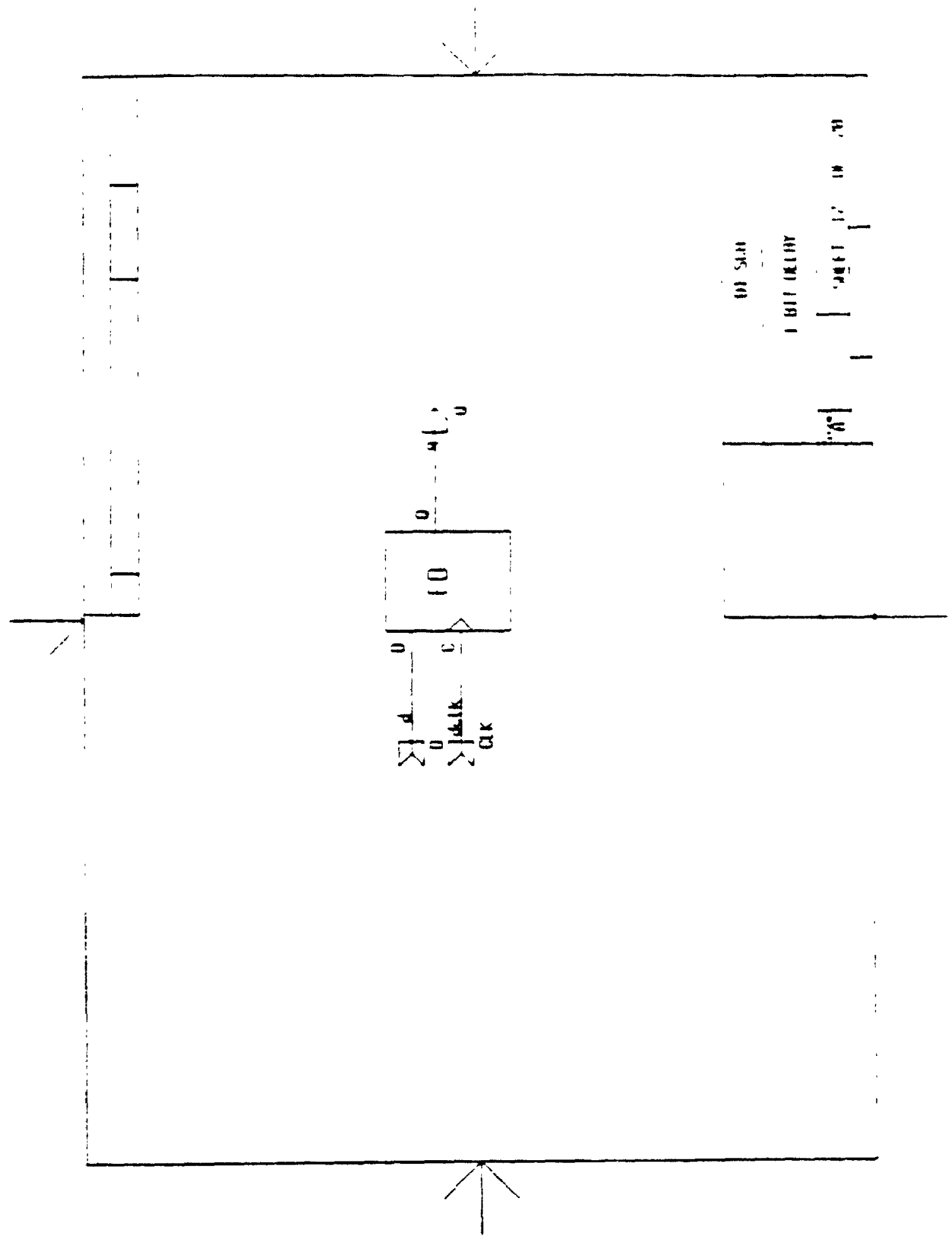

B.20 


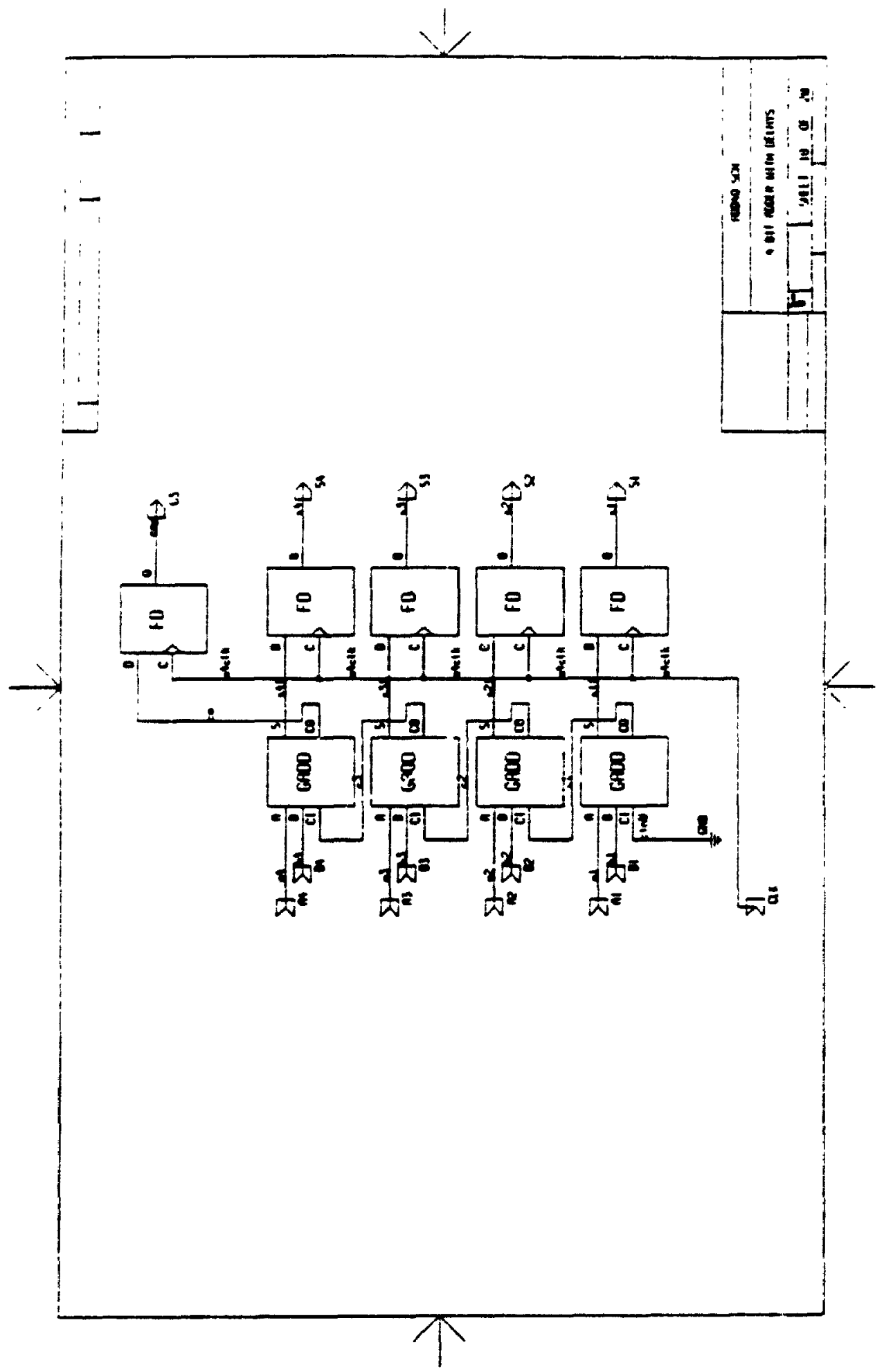




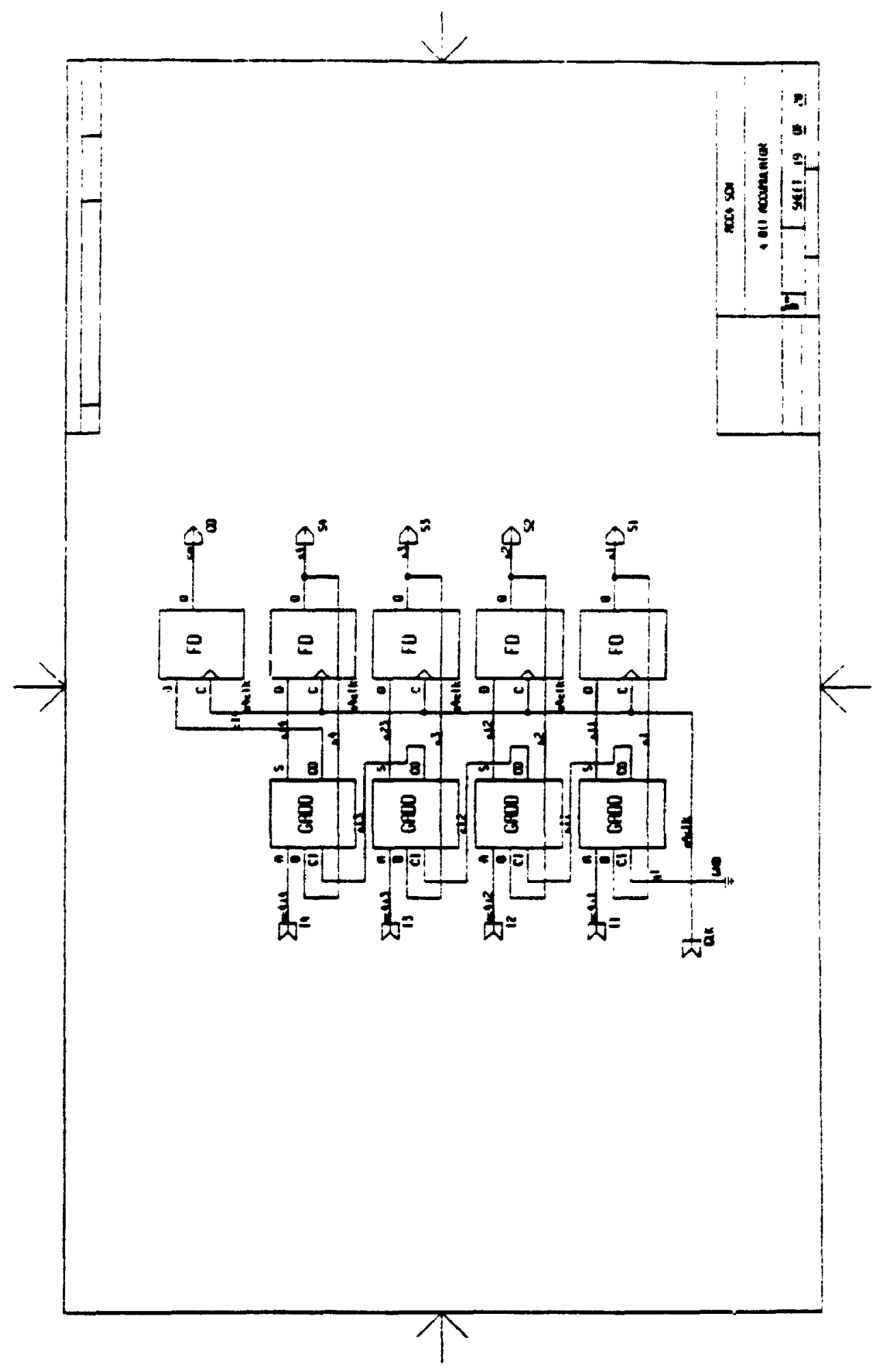




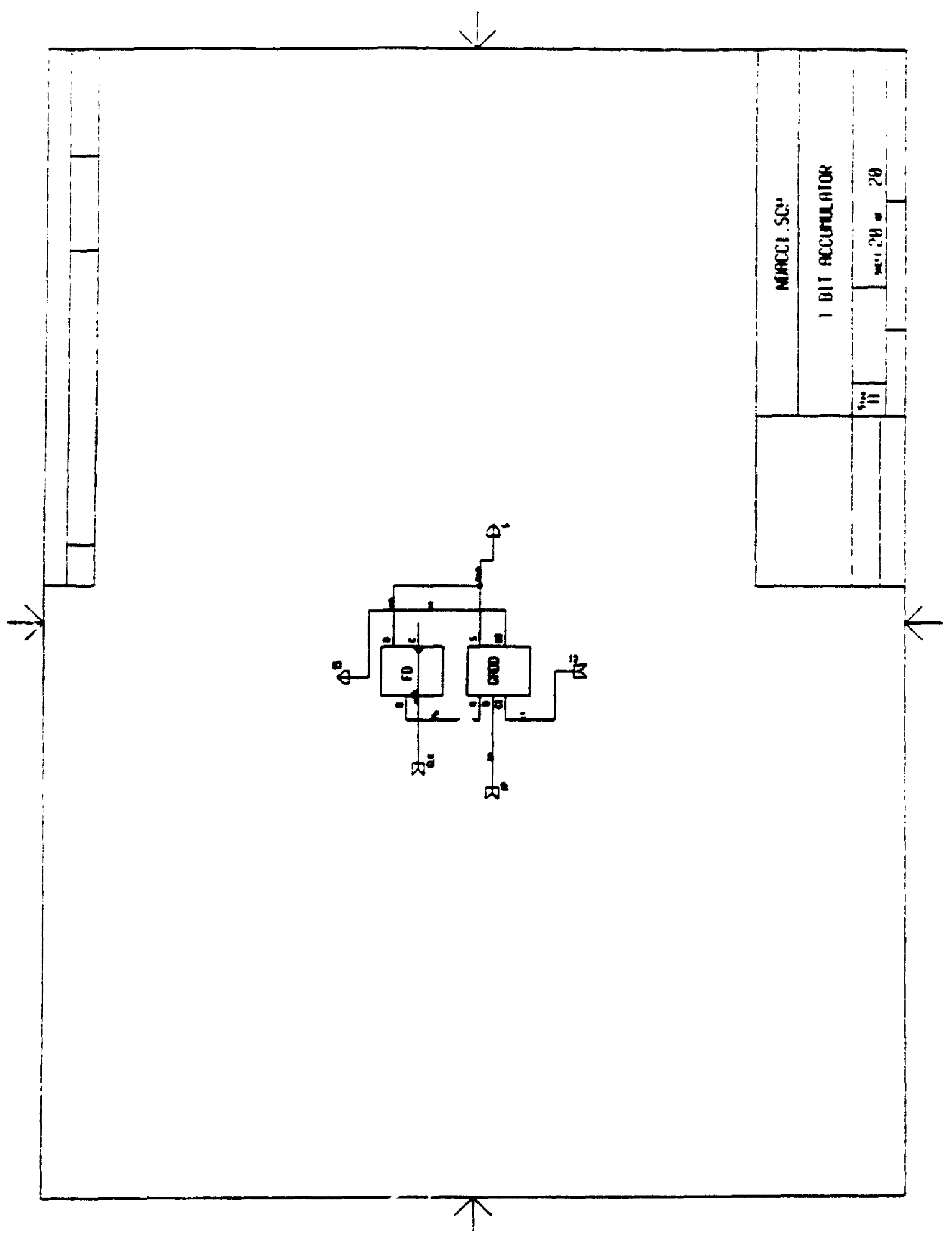

B.23 


\section{Appendix C}

\section{$\Delta \Sigma$ Frequency Discriminator Charge Pump Circuits}

This section contains a wiring diagram and parts list for both the $\Delta \Sigma$ frequency discriminator and the sub-component charge pump ciruit. An additional schematic diagram of the charge pump is provided. 


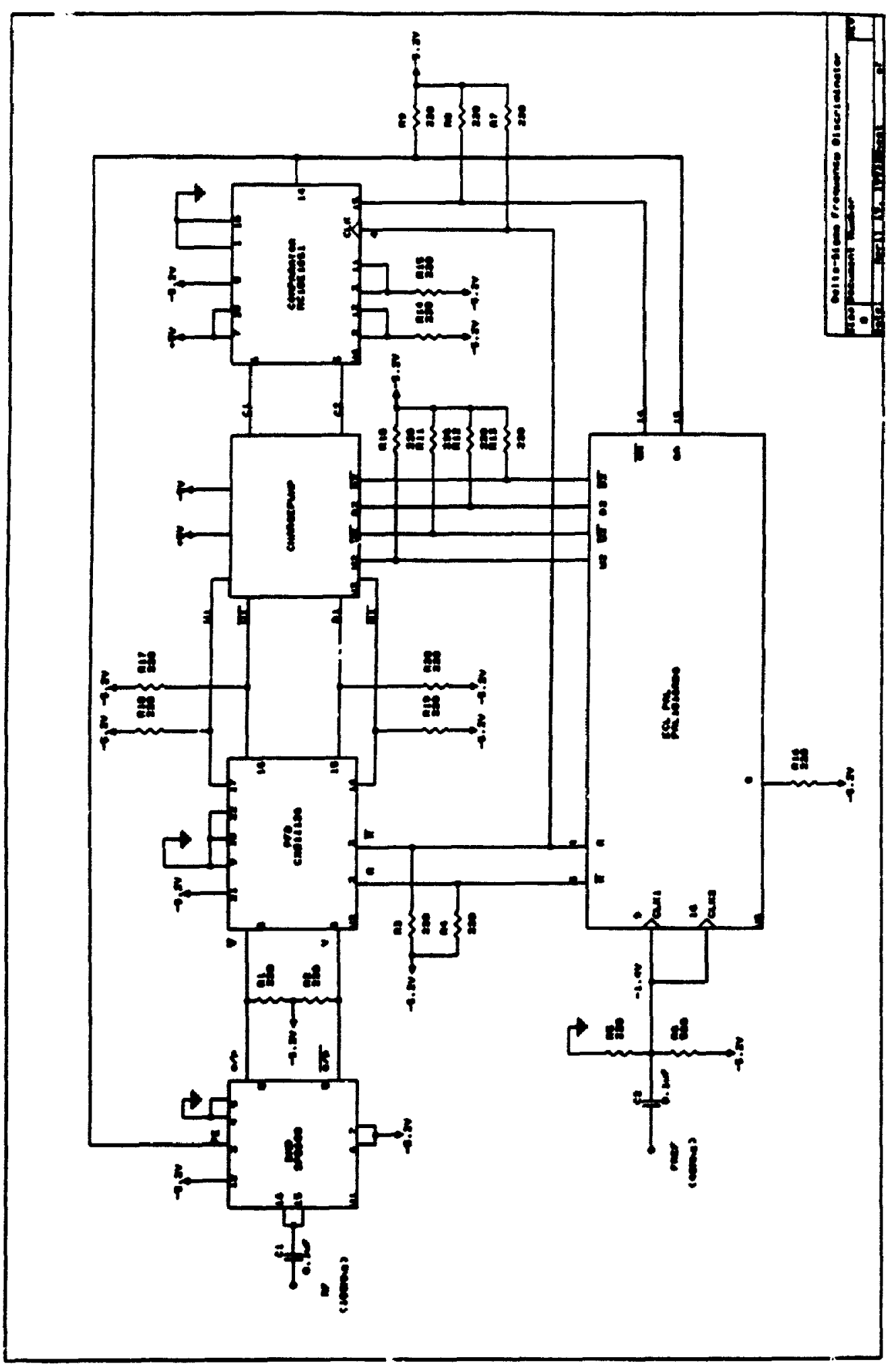

C. 2 
Frequency Discriminator Parts List April 19,1993

\begin{tabular}{llll} 
Item & Quantity & Reference & Part \\
\hline 1 & 2 & C1,C2 & $0.1 \mu f$ \\
2 & 19 & R1,R2,R3,R4,R5,R7,R8,R9, & $220 \Omega$ \\
& & R10,R11,R12,R13,R14,R15, & \\
& & R16,R17,R18,R19,R20 & \\
3 & 1 & R6 & $560 \Omega$ \\
4 & 1 & U1 & SP8680 \\
5 & 1 & U2 & CXB1112Q \\
6 & 1 & U3 & Charge Pump \\
7 & 1 & U4 & MC10E1651 \\
8 & 1 & US & PAL1016RD8
\end{tabular}




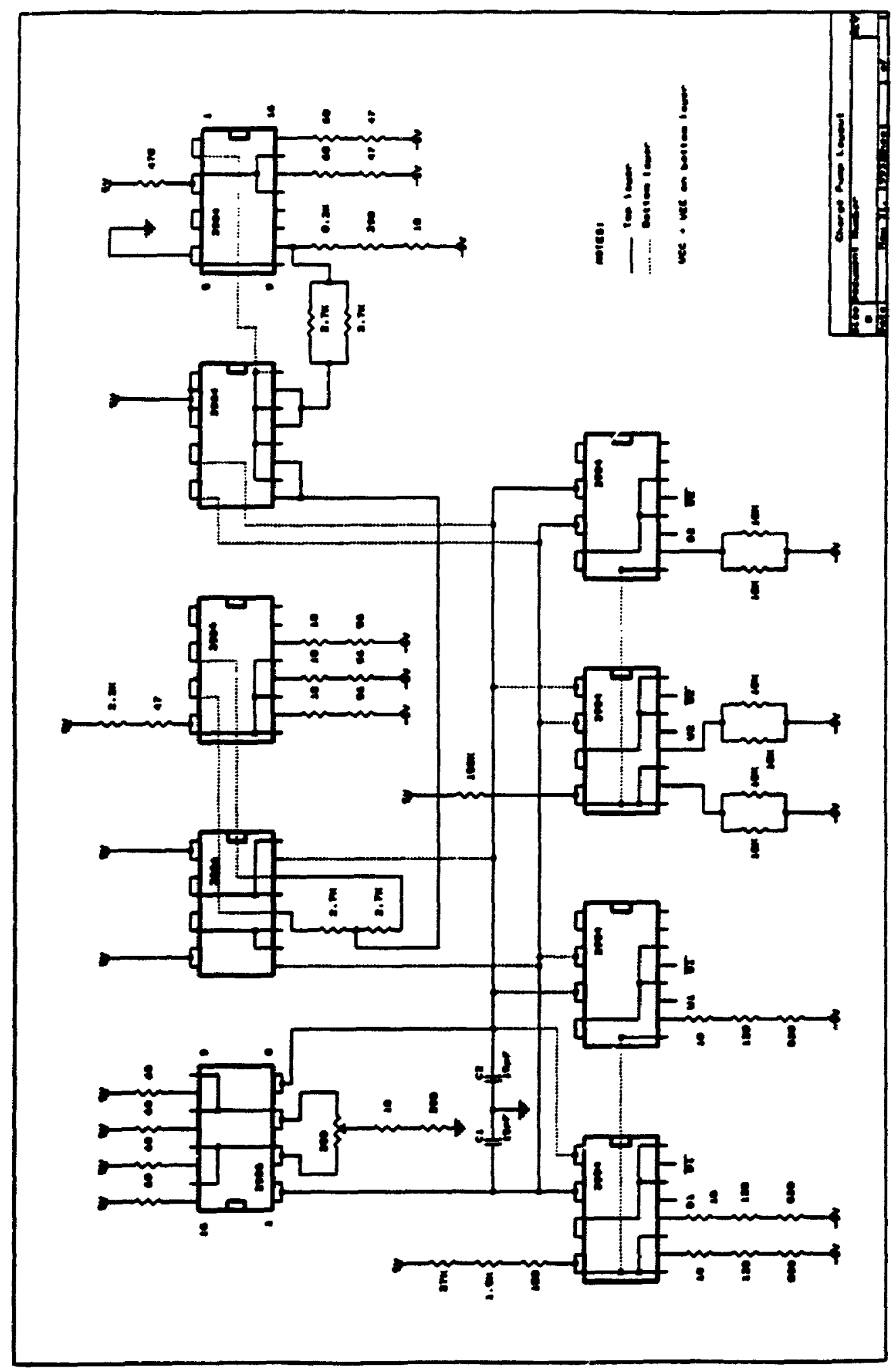


Charge Pussp Parts List July 29, 1993

Item Quantity Reference

Part

12

C1,C2 1

$15 \mathrm{pf}$

21

RI

$200 \Omega$

34

R2,R34,R36,R38

$18 \Omega$

42

R3,R40

$390 \Omega$

54

R4,R9,R12,R29

$10 \Omega$

63

RS,R10,R13

$120 \Omega$

73

R6,R11,R14

$820 \Omega$

83

$\mathbf{R} 7, \mathbf{R} 25, \mathbf{R} 27$

$47 \Omega$

91

R8

$2.2 \mathrm{~K} \Omega$

101

R15

$27 \mathrm{~K} \Omega$

111

R16

$1.5 \mathrm{~K} \Omega$

121

R17

$100 \Omega$

136

$R 18, R 19, R 20, R 21, R 24, K 26$

$68 \Omega$

141

R22

$470 \Omega$

151

R23

$150 \mathrm{k} \Omega$

161

R28

$8.2 \mathrm{k} \Omega$

174

$\mathbf{R} 30, \mathbf{R} 31, \mathbf{R} 32, \mathbf{R} 33$

$2.7 \mathrm{k} \Omega$

$18 \quad 3$

R35,R37,R39

$56 \Omega$

196

R41,R42,R43,R44,R45,R46

$10 \mathrm{k} \Omega$

209

U1

MMPQ3904 


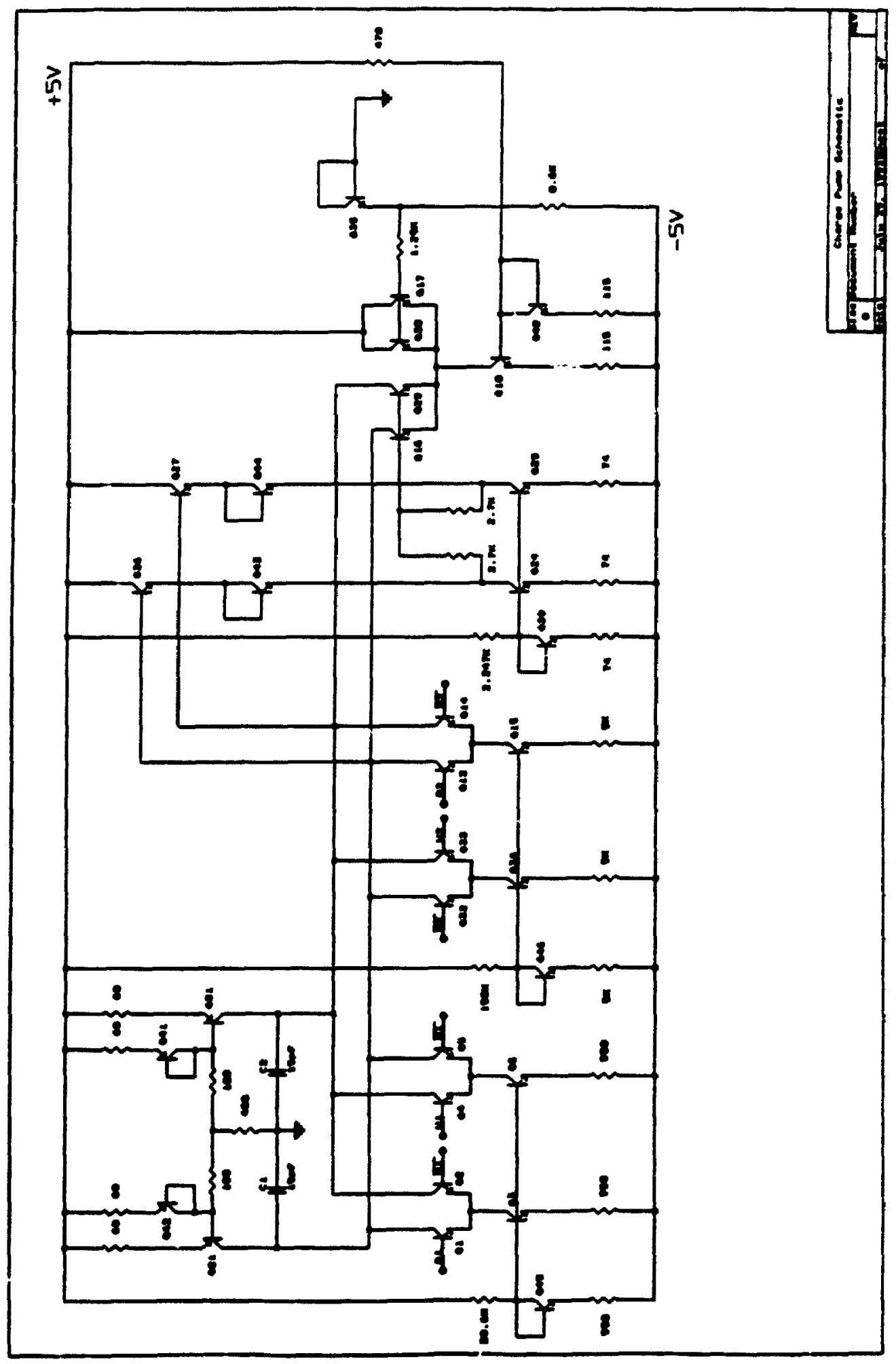




\section{Appendix D}

\section{Frequency Synthesizer Characterization Plots based upon a Broadband Sinusoidal FM Test Vector}

This section contains both frequency versus time and power spectral density plots of the individual $T_{x}$ and $R_{x}$ synthesizers operating using a broadband sinusoidal FM test vector, for several variations on FM rate and deviation. 


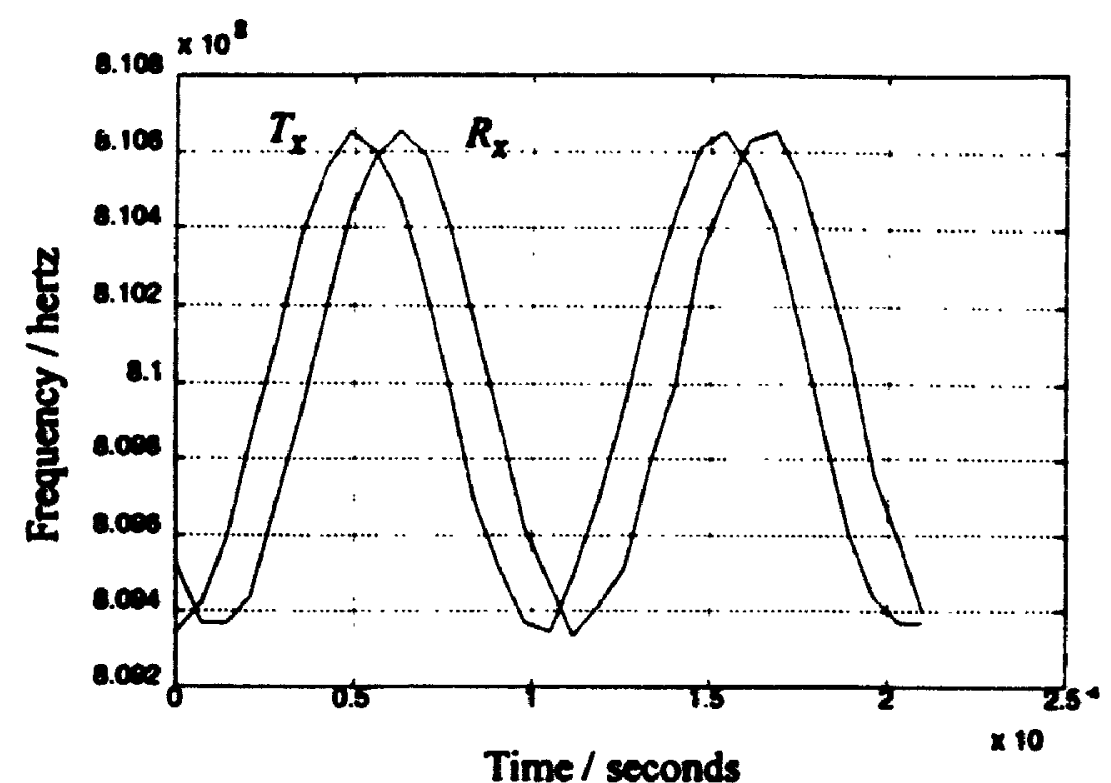

Figure D.1 : Plots of frequency versus time for the $R_{x}$ and $T_{x}$ frequency synthesizers using a $10 \mathrm{KHz}$ repetition rate and peak deviation of $\pm 625 \mathrm{KHz}$.

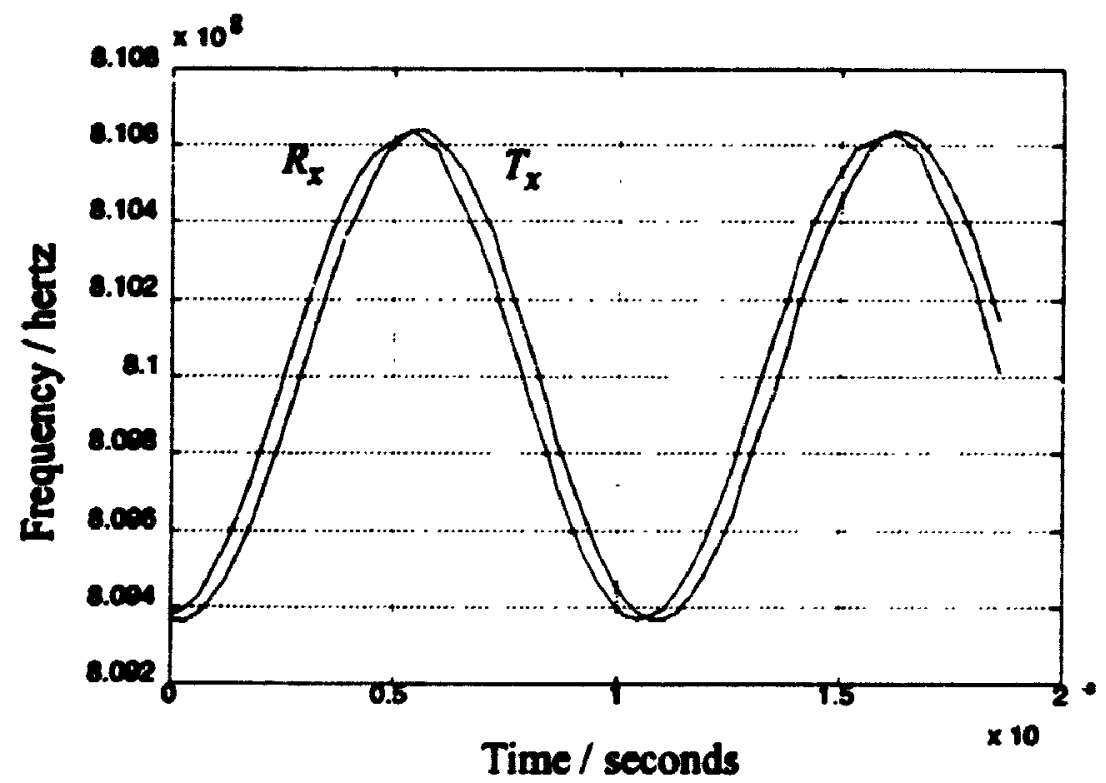

Figure D.2 : Plots of frequency versus time for the $R_{x}$ and $T_{x}$ frequency synthesizers using a $1 \mathrm{KHz}$ repetition rate and reak deviation of $\pm 625 \mathrm{KHz}$. 


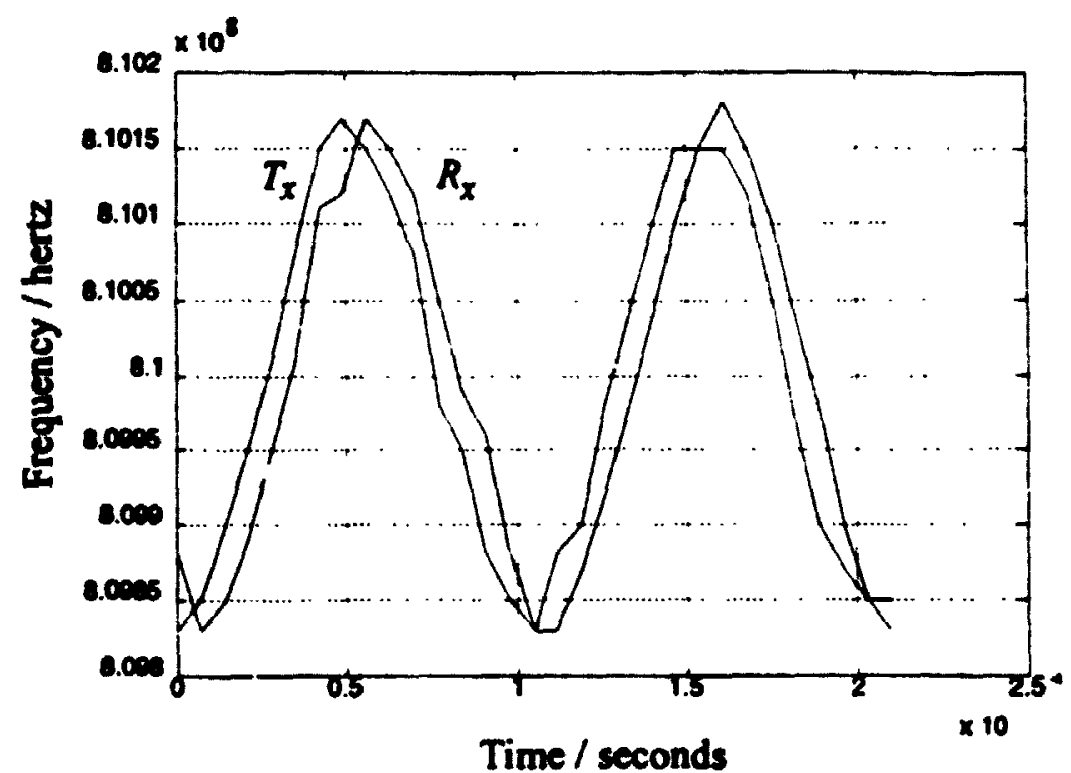

Figure D.3 : Plots of frequency versus time for the $R_{x}$ and $T_{x}$ frequency synthesizers using a $10 \mathrm{KHz}$ repetition rate and peak deviation of $\pm 156 \mathrm{KHz}$.

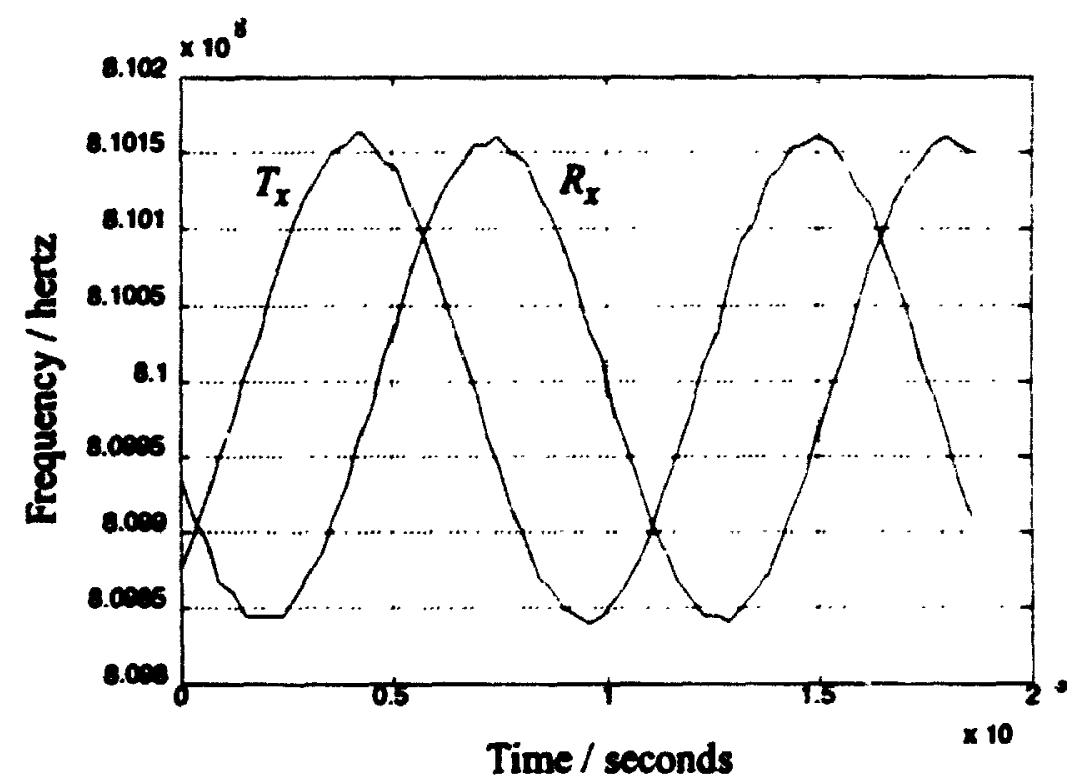

Figure D.4 : Plots of frequency versus time for the $R_{x}$ and $T_{x}$ frequency synthesizers using a I KHz repetition rate and peak deviation of $\pm 156 \mathrm{KHz}$. 


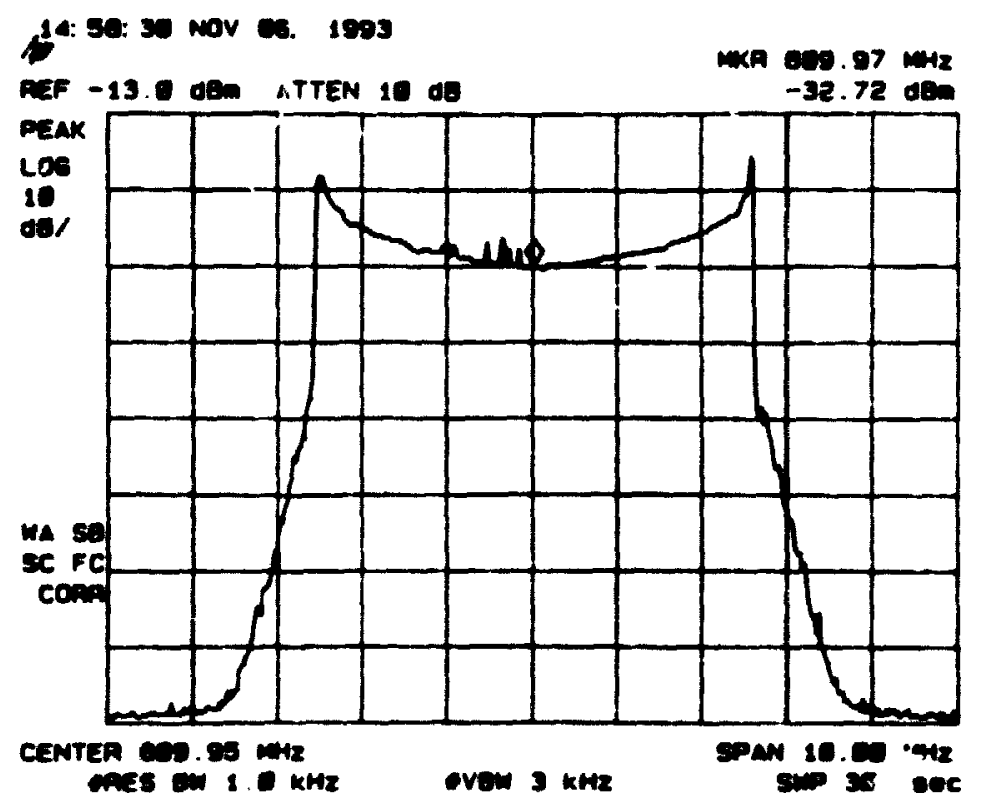

(a)

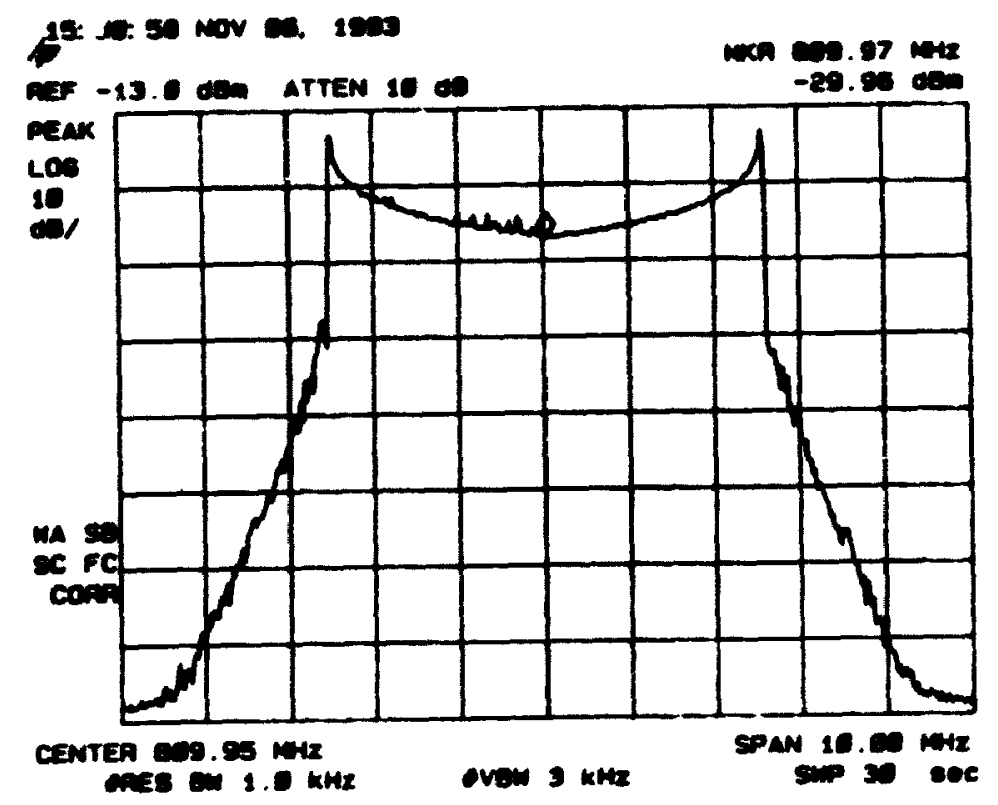

(b)

Figure D.5 : Plots of the power spectrum for a) the $R_{x}$ and b) the $T_{x}$ frequency symtherizers operating with a peak deviation of $\pm 2.5 \mathrm{MHz}$ and $1 \mathrm{KHz}$ repetition rate. 


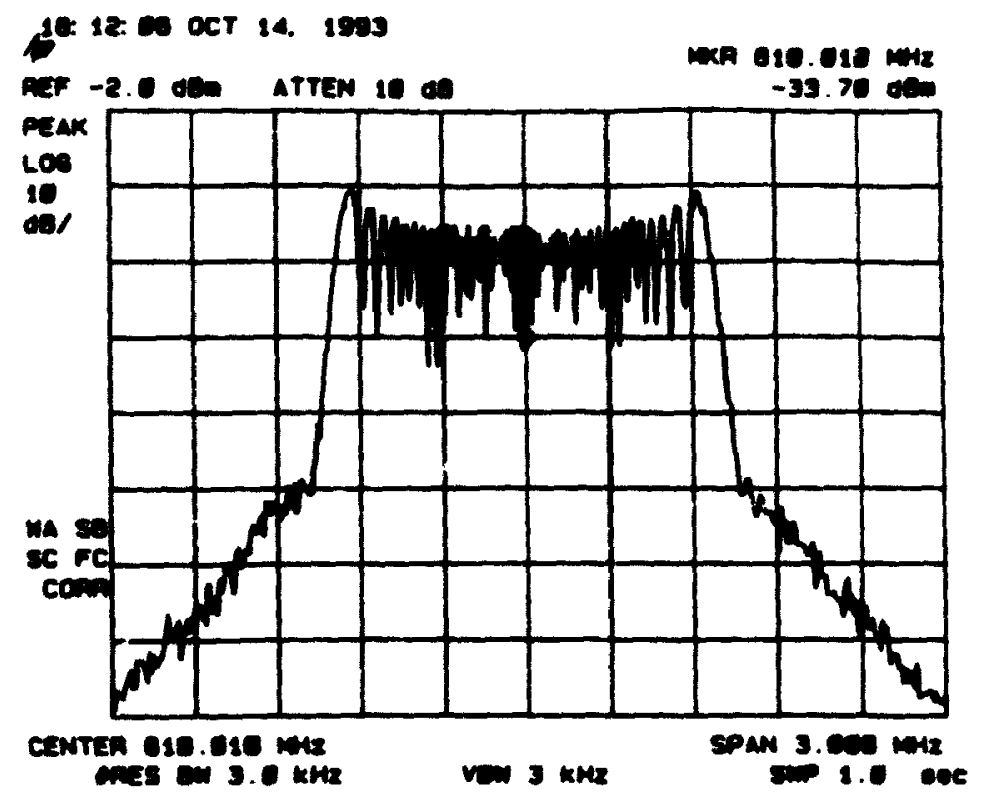

(a)

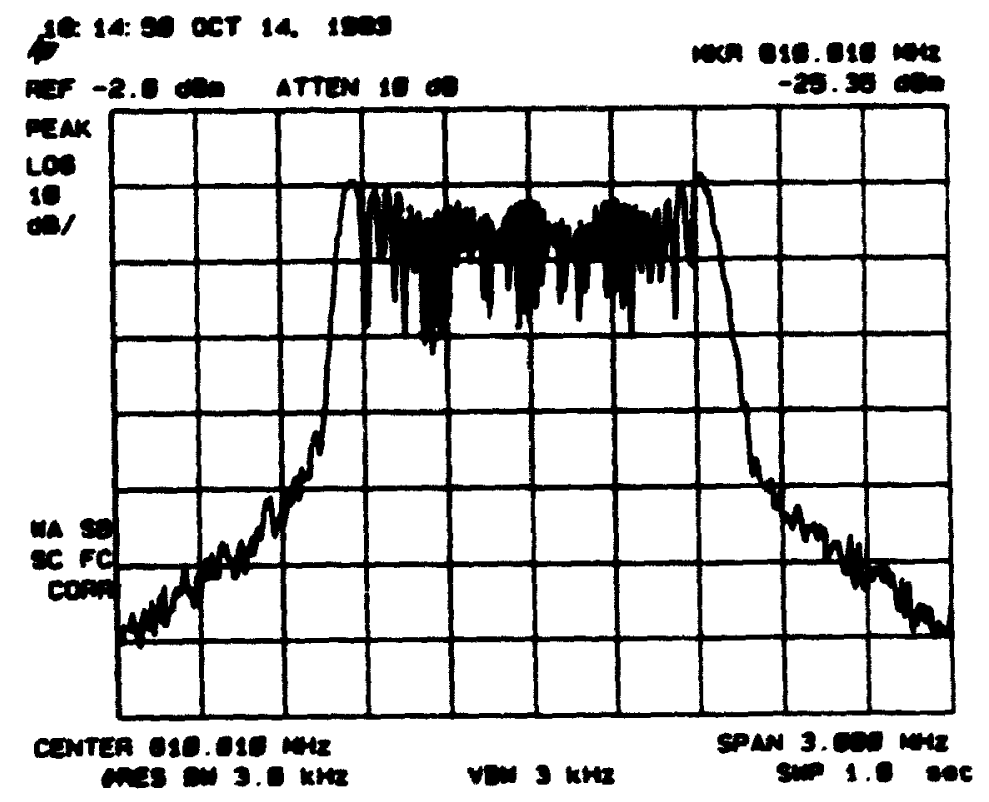

(b)

Figure D.6 : Plots of the power spectrum for $a)$ the $R_{x}$ and $b$ ) the $T_{x}$ frequency synthesizers operating with a peak deviation of $\pm 625 \mathrm{KHz}$ and $10 \mathrm{KHz}$ repetition rate. 


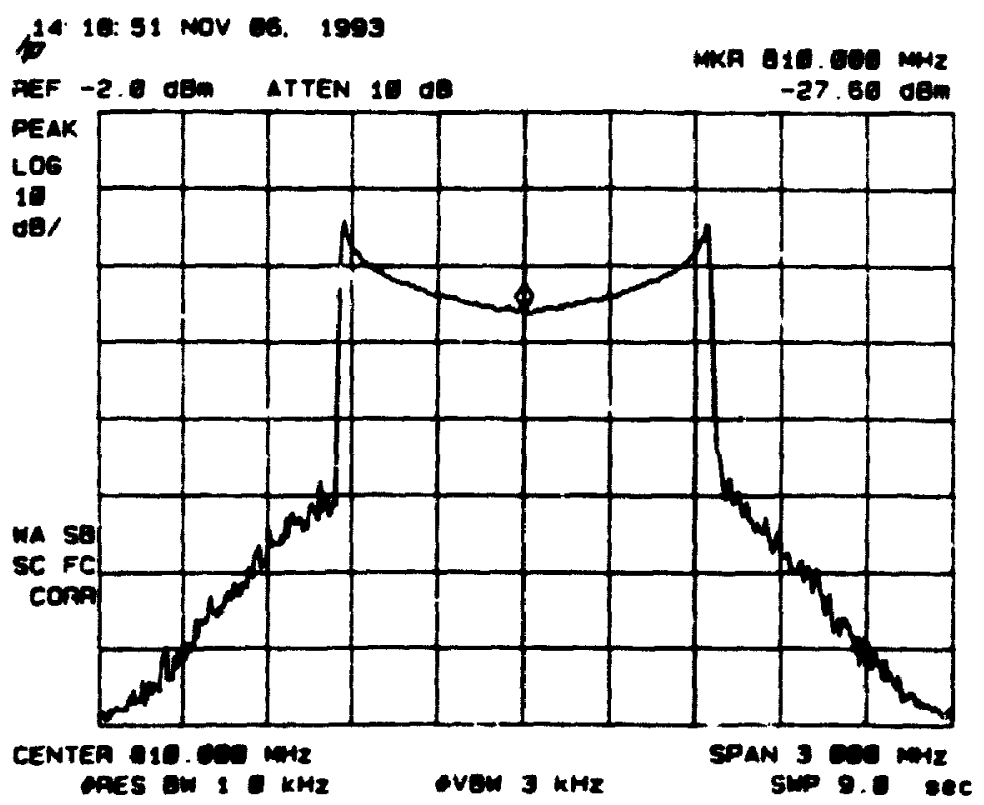

(a)

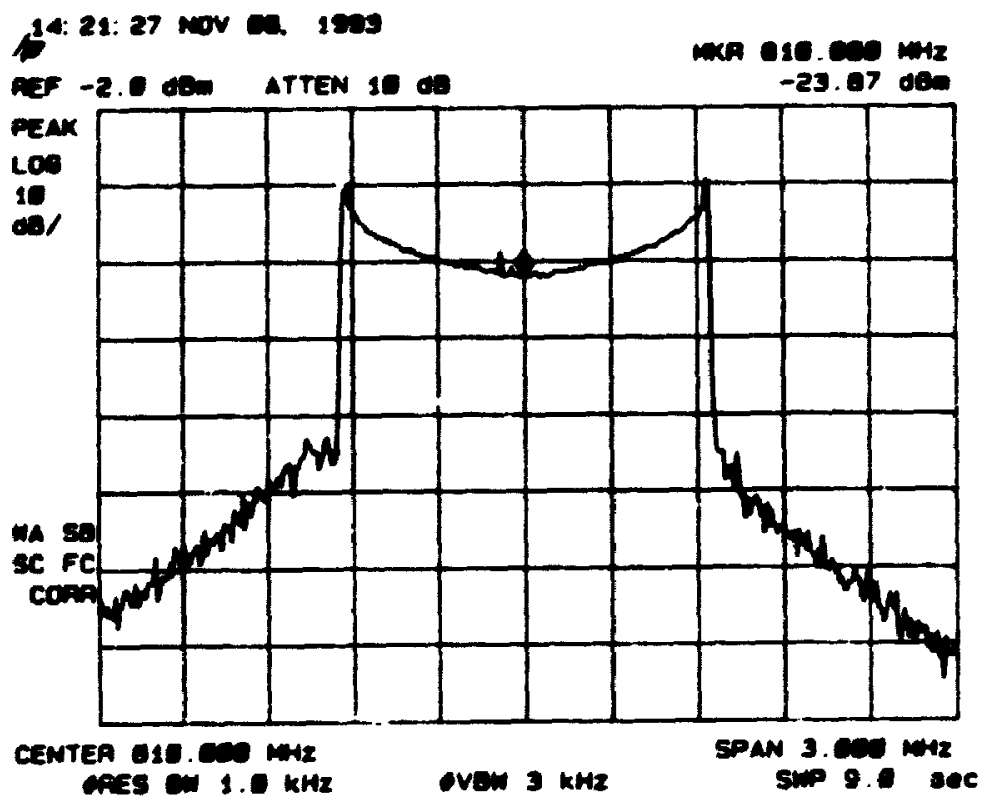

(b)

Figure D.7 : Plots of the power spectrum for a) the $R_{x}$ and b) the $T_{x}$ frequency synthesizers operating with a peak deviation of $\pm 625 \mathrm{KHz}$ and $1 \mathrm{KHz}$ repetition rate. 


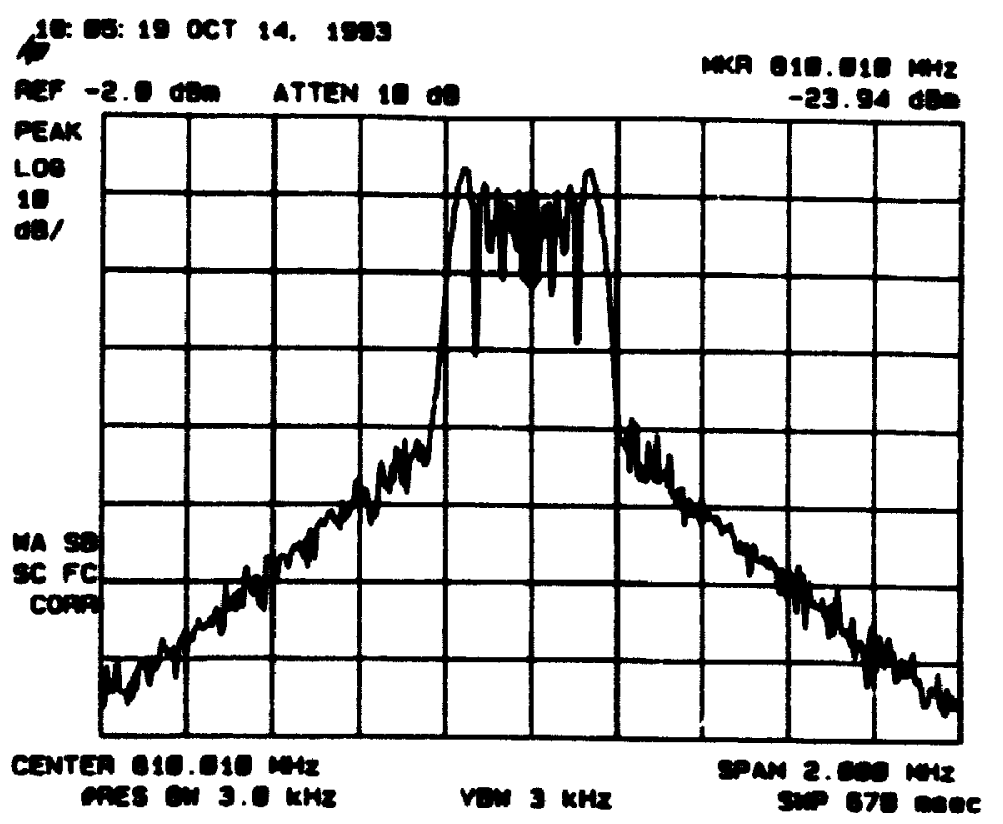

(a)

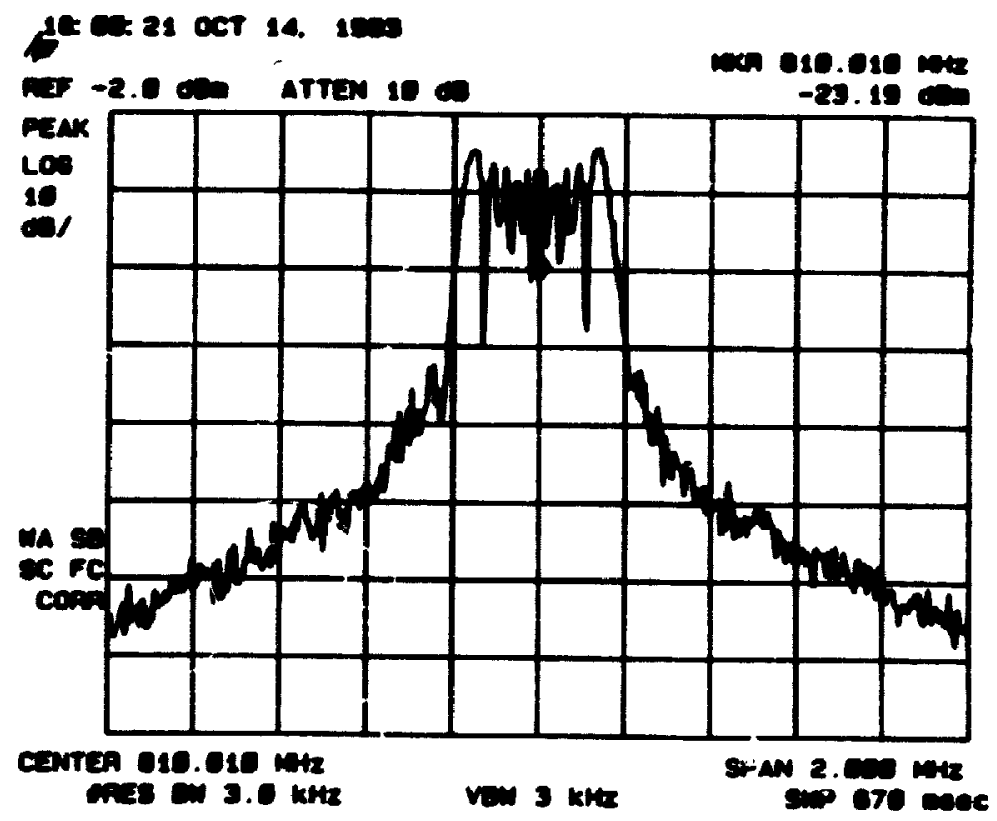

(b)

Figure D.8 : Plots of the power spectrum for a) the $R_{x}$ and $b$ ) the $T_{x}$ frequency syathesizers operating with a peak deviation of $\pm 156 \mathrm{KHz}$ and $10 \mathrm{KHz}$ repetition rate. 


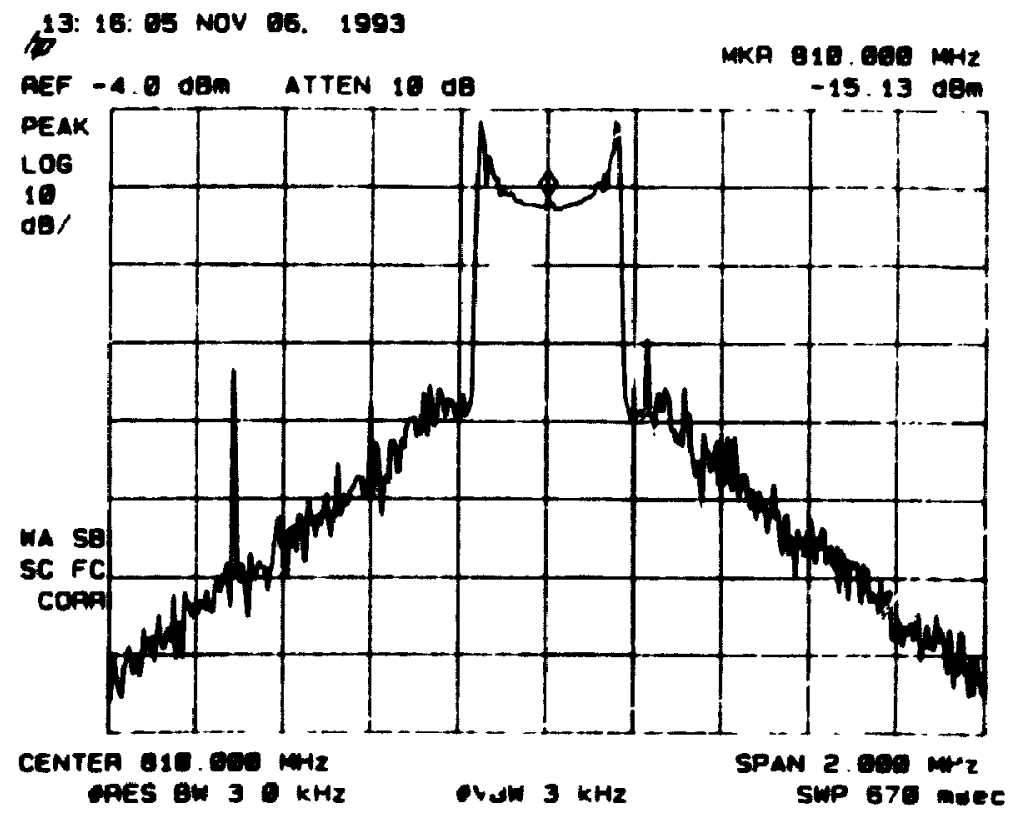

(a)

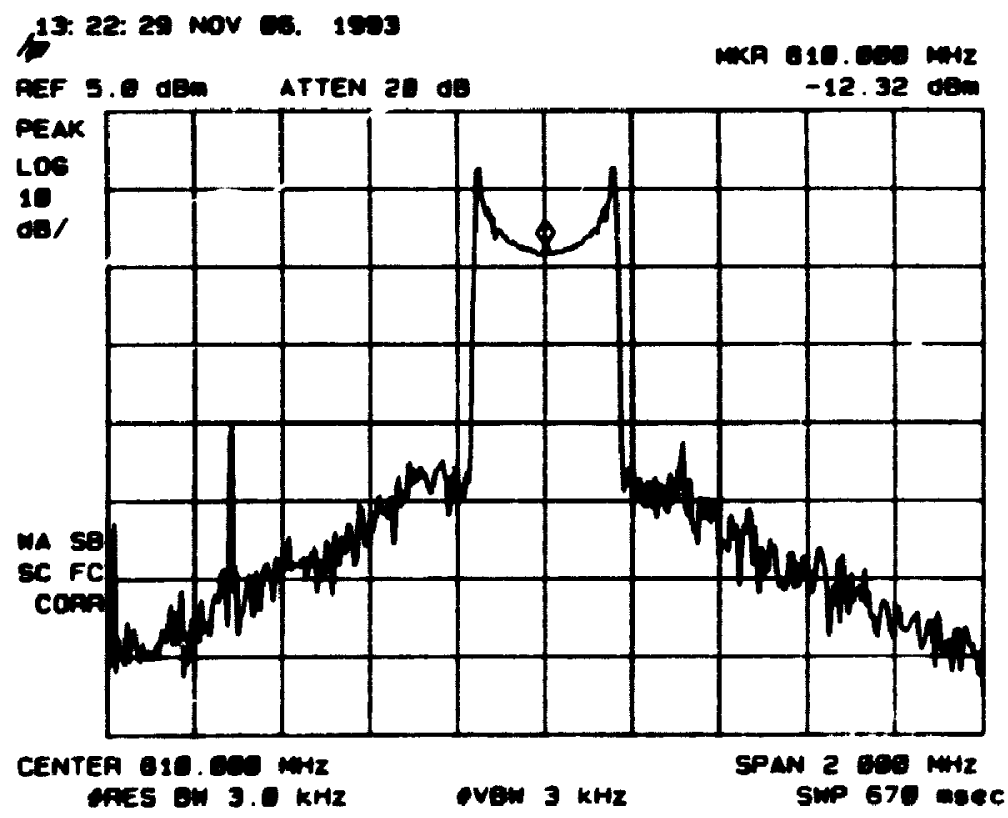

(b)

Figure D.9 : Plots of the power spectrum for a) the $R_{x}$ and b) the $T_{x}$ frequency synthesizers operating with a peak deviation of $\pm 156 \mathrm{KHz}$ and $1 \mathrm{KHz}$ repetition rate. 

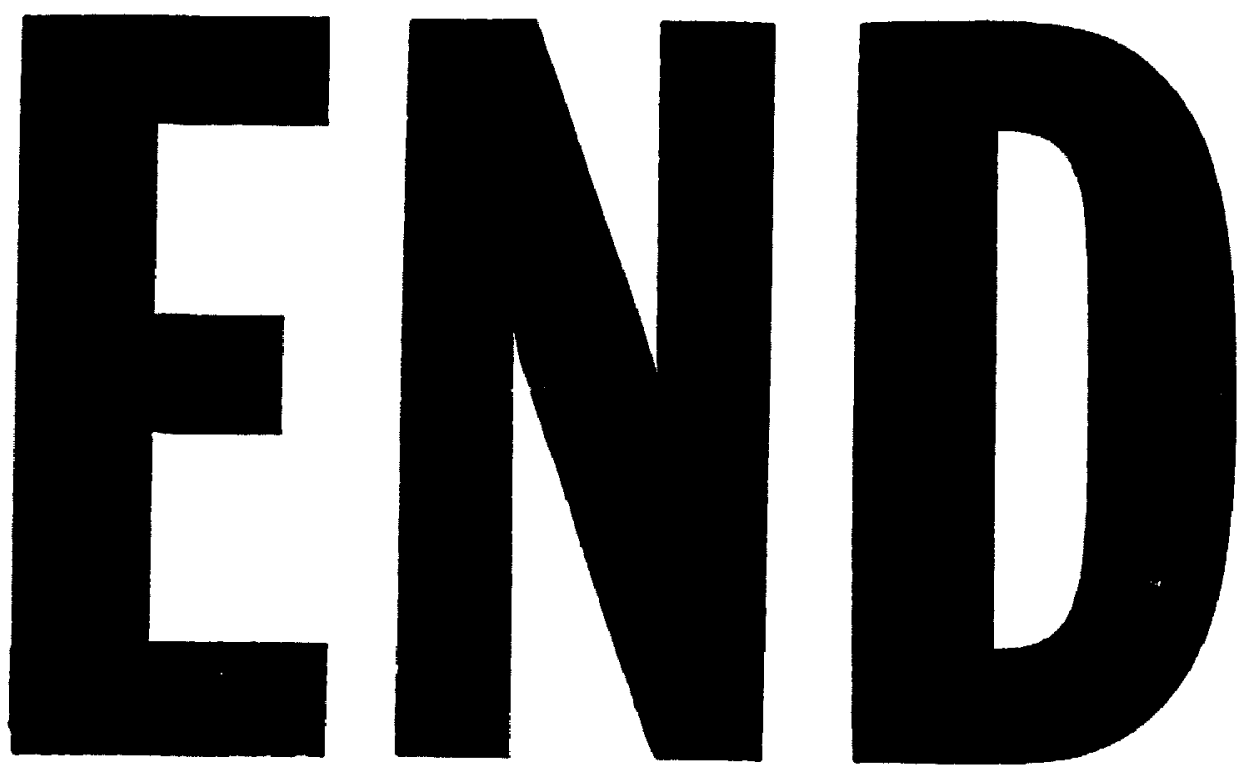

$08-11-94$
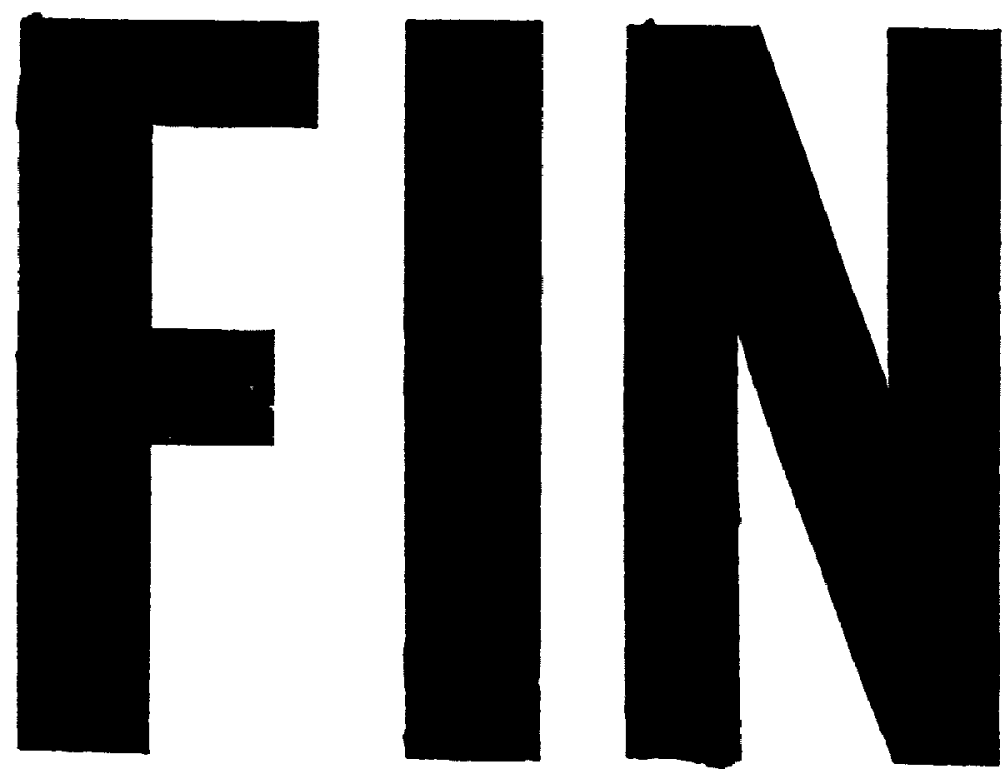